
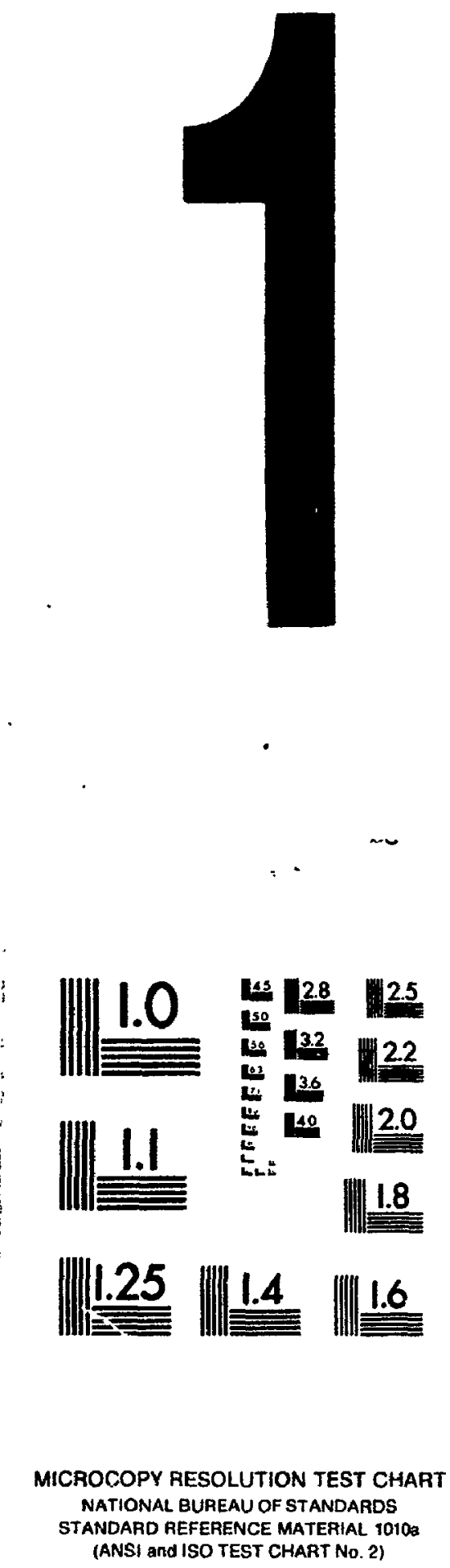
Ottawa, Canada

KIA ONA
NOTICE

The quality of this microform is heavily dependent upon the quality of the original thesis submitted for microfilming. Every effort has been made to ensure the highest quality of reproduction possible.

If pages are missing, contact the university which granted the degree.

Some pages may have indistinct print especially if the original pages were typed with a poor typewriter ribbon or if the university sent us an inferior photocopy.

Reproduction in full or in part of this microform is governed by the Canadian Copyright ACt. R.S.C. 1970, c. C-30, and subsequent amendments.

\begin{abstract}
AVIS
La qualité de cette microforme dépend grandement de la qualité de la thèse soumise au microfilmage. Nous avons tout fait pour assurer une qualité supérieure de reproduc. tion.

Sil manque des pages, veuillez comrnuniquer avec luniversité qui a contéré le grade.

La qualité d'impression de certaines pages peut laisser à désirer. surtout si les pages originales ont été dactylogra. phiées à l'aide d'un nuban usé ou si l'université nous a fait parvenir une photocopie de qualité inférieure.
\end{abstract}

La reproduction, méme partielle, de cette microforme est soumise à la Loi canadienne sur le droit d'auteur. SRC 1970 . c. C-30, et ses amendements subséquents. 


\title{
Approximate Solution for Stochastic Rendezvous Networks by Markov Chain Task-Directed Aggregation
}

by

\section{Dorina C. Petriu}

\author{
A thesis submitted to \\ the Faculty of Graduate Studies and Research \\ in partial fulfillment of \\ the requirements for the degree of \\ Doctor of Philosophy
}

Ottawa-Carleton Institute for Electrical Engineering

Faculty of Engineering

Department of Systems and Computer Engineering

Carleton University, OTTAWA K1S 5B6

April 3, 1991

(C)copyright

1991, Dorina C. Petriu 
Canadian Theses Service Service des thèses canadiennes

The author has granted an irrevocable nonexclusive licence allowing the National Library of Canada to reproduce, loan, distribute or sell copies of his/her thesis by any means and in any form or format, making this thesis available to interested persons.

The author retains ownership of the copyright in his/her thesis. Neither the thesis nor substantial extracts from it may be printed or otherwise reproduced without his/her permission.
L'auteur a accordé une licence irrévocable et non exclusive permettant à ta Bibliothíque nationale du Canada de reproduire, prater. distribuer ou vendre des copies de sa thèse de quelque manière et sous quelque forme que ce soit pour mettre des exemplaires de cette thèse à la disposition des personnes intéressées.

L'auteur conserve la propriété du droit d'auteur qui protège sa thèse. Ni la thèse ni des extraits substantiels de celle-ci ne doivent être imprimés ou autrement reproduits sans son autorisation. 
The undersigned hereby recommend to the Faculty of Graduate Studies and Research acceptance of the thesis,

\title{
Approximate Solution for Stochastic Rendezvous Networks by Markov Chain Task-Directed Aggregation
}

\author{
submitted by \\ Dorina C. Petriu
}

In partial fulfillment of the requirements

for the degree of Doctor of Philosophy

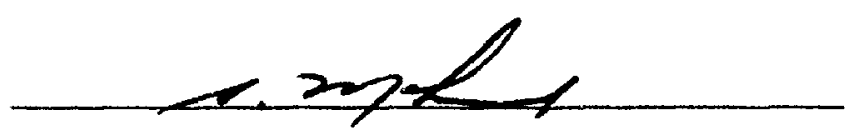

Chair, Department of Systems and Computer Engineering

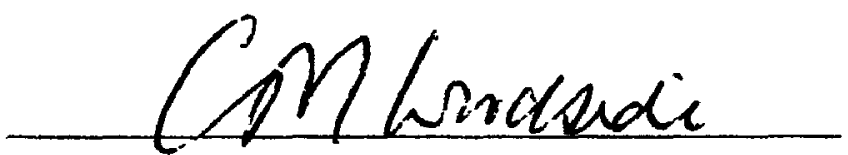

Thesis Supervisor

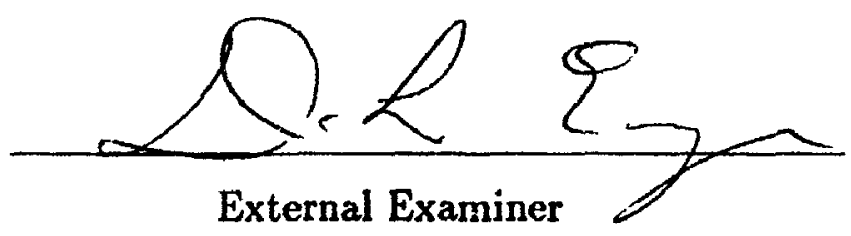

Carleton University

May 10,1991 


\section{Abstract}

Modelling concurrent software is a challenging direction of research in the field of anilytical performance modelling. Stochastic Rendezvous Network (SRVN) is a new modelling method, recently developed at Carleton University for software systems with rendezvous (RV) communication. This thesis was motivated by two main factors: the search for SRVN solution methods with a better accuracy, and the need for a more rigorous theoretical foundation to replace the ad hoc collection of heuristic approximations used in the previous SRVN solutions.

The thesis defines and studies the properties of "task-directed aggregation" (TDA) of the Markov Chains (MC) models for tasking systems with synchronous communication, where the aggregation is done in function of the task states. TDA is applied to the MC of a simple: client/server SRVN with one server and $n$ clients, proving that the transition rate submatrices of the aggregated MCs have the same form for any $n$. Based on this result, a set of equations for the arrival instant probabilities are derived from the balance equations of a set of aggregated MCs. These equations are then used in a new algorithm TDA/MVA(1), based on the MVA framework from the Queueing Network theory. An extended algorithm TDA/MVA(2) is developed for a case arisen from practical needs, where the clients are: allowed to change the class of requests sent to the server. The two algorithms for simple: client/server SRVN are integrated into a new iterative decomposition algorithm TDA/DE: for complex SRVN models with any number of servers and multi-layered service. The assessment of accuracy and convergenco for the new algorithms is done through experimental results. 


\section{Acknowledgements}

I would like to express my deep gratitude to my supervisor Dr. C. M. Woodside, who led me to this interesting and challenging research area, for his guidance and consistent support throughout the course of this work. His experienced advice and encouragement in the preparation of this thesis are thankfully acknowledged.

I would like to offer my appreciation to the members of our performance research group, and especiaily to Dr. J. E. Neilson, for many engaging and valuable discussions that were a source of inspiration for my work.

I would like to thank the external examiner, Dr. D. Eager from University of Saskatchewan, and the examining board members, Dr. M. K. Krieger from University of Ottawa, Dr. N. W. Dawes and Dr. T. Kwasniewski from Carleton University, for their careful reading of my thesis, and for providing valuable suggestions.

I also wish to take this opportunity to thank my husband Emil, for his constant encouragement and support, and my children Dorin and Mihaela, for their patience and understanding during the many hours I devoted to working.

Financial assistance provided by the Ontario Ministry of Colleges and Universities through its U.R.I.F. grant is gratefully acknowledged. This work has its origins in a contract with Bell Canada, and benefited from the stimulation of practical problems posed in that study. 


\section{Contents}

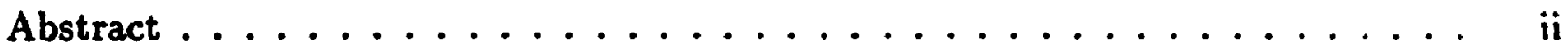

Acknowledgements ......................... iii

List of figures $\ldots \ldots \ldots \ldots \ldots \ldots \ldots \ldots \ldots \ldots \ldots$ vii

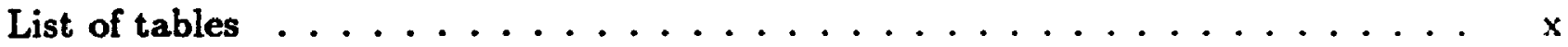

List of symbols and terminology $\ldots \ldots \ldots \ldots \ldots \ldots \ldots \ldots$ xi

1 Introduction 1

1.1 Research Motivation . . . . . . . . . . . . . . . . 1

1.1.1 Software abstraction for performance modelling . . . . . . . . . 3

1.1.2 Software servers and client/server systerns . . . . . . . . . . 6

1.1.3 Comparison of main analytical 1 ıodelling methoàs . . . . . . . . \& \&

1.1.4 Stochastic Rendezvous Networks . . . . . . . . . . . . . 10

1.2 Research Goals and Contributions . . . . . . . . . . . . . . . 14

1.2.1 Long and Short Term Goals . . . . . . . . . . . . . . . 14

1.2 .2 Contributions . . . . . . . . . . . . . . 15

1.3 Thesis Overview . . . . . . . . . . . . . . . 16

2 Literature review $\quad 18$

2.1 Queueing Network models and solution methods . . . . . . . . . . . 18

2.1.1 QNs with product form solution . . . . . . . . . . . 19

2.1.2 Exact computational methods for product form QNs . . . . . . 21

2.1.3 Approximate solution methods for product form QNs . . . . . . 23

2.1 .4 Non-product form $\mathrm{QNs} \ldots \ldots \ldots \ldots$

2.1.5 General discussion about approximate iterative methods for QNs . . 38

2.2 Solution methods at the Markov chain level $\ldots \ldots \ldots \ldots$. . . . . . . II 
2.2.1 Markov chain aggregation-disaggregation $\ldots \ldots \ldots . \ldots . \ldots 40$

2.2.2 Nearly decomposable systems . . . . . . . . . . . . . . 43

2.3 Petri Net models and solution methods . . . . . . . . . . . . . 45

2.3.1 Stochastic and Timed Petri Nets . . . . . . . . . . . . 46

2.3.2 Approximate solution methods for Stochastic Petri Nets . . . . . . 49

2.4 Literature Review Conclusion . . . . . . . . . . . . . . . . 51

3 Markov Chain Task-Directed Aggregation 54

3.1 Formal Definition of an SRVN Model . . . . . . . . . . . . . . 54

3.1.1 Heuristic MVA solution method for SRVN . . . . . . . . . 57

3.2 Task-Directed Aggregation . . . . . . . . . . . . . . 60

3.2.1 State Vector of a Tasking System . . . . . . . . . . . . . 60 60

3.2.2 TDA-1 and TDA-2: definitions and properties . . . . . . . . 62

3.2.3 Task system decomposition based on TDA . . . . . . . . . 75

4 Arrival Instant Probabilities for Simple Client/Server SRVN

4.1 Introduction . . . . . . . . . . . . . . . . 79

4.2 Markov Chain model of a simple client/server SRVN . . . . . . . . 81

4.2.1 MC of a simple client/server SRVN with two clients . . . . . . 81

4.2.2 MC of a simple client/server SRVN with $n>2$ clients . . . . . . 84

4.3 Task-directed aggregation of the MC for a simple client/server SRVN . . . 89

4.3.1 MC aggregation for two designated tasks . . . . . . . . . . 90

4.3.2 MC aggregation for three designated tasks . . . . . . . . . 98

4.4 Derivation of equations for arrival instant probabilities . . . . . . . . . 102

4.5 Algorithm and experimental results . . . . . . . . . . . . . 113

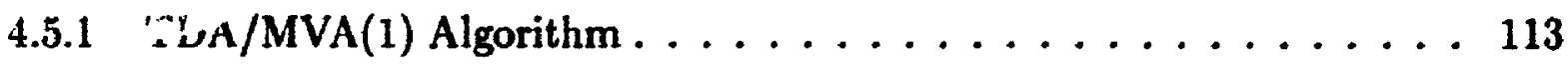

4.5.2 Experimental results . . . . . . . . . . . . . . . 114

5 Clients with Multi-class Requests in Simple Client/Server SRVN 119

5.1 Clients with multi-class requests . . . . . . . . . . . . . . . 119

5.1 .1 Task Dependencies . . . . . . . . . . . . . . . 120

5.2 Arrival probabilities for clients with multi-class requests . . . . . . . . . 124 
5.3 Algorithm and experimental results $\ldots \ldots \ldots \ldots \ldots \ldots \ldots$

5.3.1 TDA/MVA(2) Algorithm . . . . . . . . . . . . . 1:36

53.2 Experimental results $\ldots \ldots \ldots \ldots \ldots \ldots \ldots$

6 TDA-based Decomposition of Complex SRVN Models $\quad \mathbf{1 . 4 0}$

6.1 Decomposition of SRVN models into client/server submodels . . . . . . . 110 6.1.1 TDA/DEC Algorithm ...................... Ii

6.2 Application: SRVN model for an hierarchical database . . . . . . . . (50)

6.3 Study of software bottleneck in multi-layered systems . . . . . . . . 15.5

$\begin{array}{lll}7 \text { Conclusions } & 161\end{array}$

$\begin{array}{ll}\text { A Nondiagonal submatrices of } Q^{\prime \prime}(n) & 166\end{array}$

B Test suites for simple client/server SRVN $\quad 168$

$\begin{array}{ll}\text { Bibliography } & 191\end{array}$ 


\section{List of Figures}

1.1 QN model of a time sharing system . . . . . . . . . . . . . . 4

1.2 Flow model for concurrent software . . . . . . . . . . . . 5

1.3 PN model for the flow graph model from Figure $1.2 \ldots \ldots \ldots$

1.4 Example of an Sk'N model . . . . . . . . . . . . . . . 12

2.1 Markov Chain aggregation $\ldots \ldots \ldots \ldots \ldots \ldots \ldots \ldots \ldots$

3.1 State machine for an accepting task $T_{i}$ with entries $\ldots \ldots \ldots \ldots$

3.2 TDA-1 aggregation where state $\alpha_{i}$ of $T_{i}$ is active $\left(T_{i} \in D\right.$, and $\left.|D|>1\right) \ldots 64$

3.3 (a) Task state machine and (b) TDA-1 aggregation where $T_{i}$ in state $\alpha_{i}$ chooses the next state with given probabilities $\left(T_{i} \in D\right.$, and $\left.|D|>1\right) \quad \ldots \quad 67$

3.4 TDA-1 where state $\tau_{i}$ of $T_{i}$ is passive, and $T_{i}$ is the unique designated task . 68

3.5 TDA-1 aggregation where state $\tau_{i}$ of $T_{i}$ is passive $\left(T_{i} \in D\right.$, and $\left.|D|>1\right) \ldots \quad 70$

3.6 TDA-2 aggregation for $T_{i}:$ (a) at state machine level, and (b) at MC level, where the MC is aggregated by TDA-1 with $T_{i}$ as unique designated task . . 72

3.7 TDA-2 aggregation for $T_{i}$ : effect at $\mathrm{MC}$ level, where the $\mathrm{MC}$ is aggregated by TDA-1, with $T_{i} \in D$ and $|D|>1 \ldots \ldots \ldots \ldots$

4.1 Simple client/server SRVN with $n$ clients and one server $\ldots \ldots \ldots$

4.2 Markov Chain for a simple client/server SRVN with two clients . . . . . 82

4.3 Groups of states of the MC for a simple client/server SRVN with two clients 83

4.4 Groups of states of the MC for a simple client/server SRVN with $n$ clients - 85

4.5 Group $\mathcal{G}_{41}$ of the MC for a simple client/server SRVN with 3 clients . . . . 87

4.6 Aggregated MC of a simple client/server SRVN with two clients, for $D=\{i, s\} 91$

4.7 Aggregation for $D=\{2,4\}$ of group $G_{41}$ from Figure $4.5 \ldots \ldots \ldots$ 
4.8 Aggregated MC of a simple client/server SRVN with $n$ clients, for $D=\{i, s\} \quad 96$

4.9 Aggregation for $D=\{1,2,4\}$ of group $\boldsymbol{G}_{41}$ from Figure $4.5 \ldots \ldots$. . . . . . (!)

4.10 Group of aggregated states $\mathcal{G}_{\text {sk }}^{\prime \prime}$ where a non-designated task $T_{k}$ is in sorvici. . 11:.

4.11 Maximum throughput error/case in function of client/server and server uIt-

balance for test suite CS5 .....................116

4.12 Maximum throughput error/case in function of server utilization for test. suite.

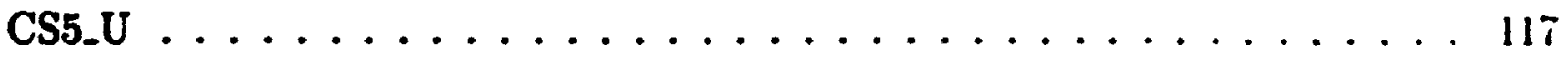

5.1 Example for test suite MR3 ..................... 120

5.2 Example for test suite MR4 . . . . . . . . . . . . . . . . 121

5.3 Example for test suite MR5 . . . . . . . . . . . . . . . . . . 121

5.4 Example of an SRVN model with mutually exclusive requests . . . . . . . . 122

5.5 Abstract representation of a client with multi-class requests . . . . . . . 12.t

5.6 Abstract representation of a server . . . . . . . . . . . . . 125

5.7 Groups of $\mathrm{MC}$ states corresponding to different request classes . . . . . . . 126

5.8 Group $\mathcal{G}_{c s}$ of $\mathrm{MC}$ states aggregated for $D=\{i, j, s\}$, where $a, b \in \mathcal{R}_{\mathrm{s},}, \quad c, d \in$

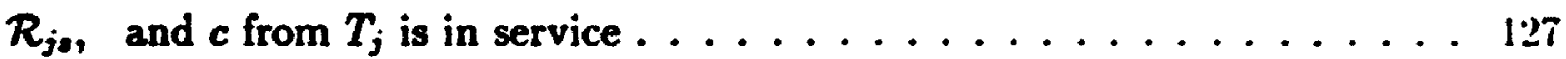

5.9 Group $\mathcal{G}_{b,}$ of $\mathrm{MC}$ states aggregated for $D=\{i, s\}$, where $a, b \in \mathcal{R}_{1,}$, and $b$ from $T_{i}$ is in service . . . . . . . . . . . . . . . . . I?!)

5.10 Group $\mathcal{G}_{c s}$ of $\mathrm{MC}$ states aggregated for $D=\{i, s\}$, where $a \in \mathcal{R}_{s,}, c \in \mathcal{R}_{, n}$, and $c$ from $T_{j}$ is in service . . . . . . . . . . . . . . 1:30

5.11 Group $\mathcal{G}_{c}$ of $\mathrm{MC}$ states aggregated for $D=\{i, j, s\}$, where $a, c \in \mathcal{R}_{i s,} b, d \in$ $\mathcal{R}_{j a}$, and $c$ from $T_{i}$ is in service . . . . . . . . . . .

5.12 Group $\mathcal{G}_{k,}$ of MC states aggregated for $D=\{i, j, s\}$, where $a \in \mathcal{R}_{i s,} d \in$ $\mathcal{R}_{j s,}, c \in \mathcal{R}_{k s,}$ and $c$ from $T_{k} k \neq i, j$ is in service . . . . . . . . 13ij

6.1 Decomposition of an SRVN model into submodels . . . . . . . . . . . 1.12

6.2 TDA-2 applied to (a) Reference task, and (b) Non-reference task to obtain (c) Abstract client with single-class requests . . . . . . . . . . . . 113

6.3 TDA-2 applied to (a) Reference task, and (b) Non-reference task to obtais (c) Abstract client with multi-class requests . . . . . . . . . . . 117

6.4 SRVN model for a hierarchical database: test suite HDB1 . . . . . . . . . . 1:0) 
6.j SRVN r.odel for a hierarchical database: test suite HDB2 . . . . . . . 151

6.6 Exact and approximate utilization for tasks and processors . . . . . . 156

6.7 Utilization components for the bottleneck task T4 . . . . . . . . . 157

6.8 First software solution to alleviate the software bottleneck: shared server clones on the same processor . . . . . . . . . . . . . 158

6.9) Second software solution to alleviate the software bottleneck: dedicated server clones on the same processor . . . . . . . . . . . . . 158

6.10 Transformation of the model from Figure 6.8 tc represent the shared processor as an SRVN task ...................... 159 


\section{List of Tables}

4.1 Markov Chain size of a client/server model . . . . . . . . . . . . s8

4.2 Comparison of the mean absolute percentage error obtained with TDA/MVA(1) and Heuristic MVA algorithms for test suites CS3-CS5 (results given in ap-

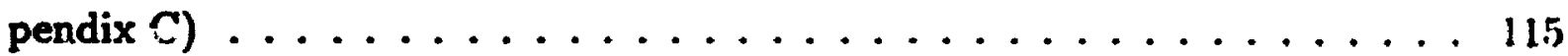

4.3 TDA/MVA(1) Algorithm accuracy with respect to model unbalance . . . . 116

5.1 Comparison of mean throughput error obtained with the old and new approximate SRVN algorithms . . . . . . . . . . . . . 138

5.2 Algorithm accuracy for suite MR5 with respect to model unbalance . . . . 139

6.1 Execution Times of Servers T7, T8, T9 for a group from suite IIDB1, HDB2 152

6.2 Suite HDB1, HDB2 - Mean Number of RV requests (user T6 present only in HDB2) . . . . . . . . . . . . . . . . . . 153

6.3 Relative throughput and utilization error for test suite HDB1, HDB2 . . . 154

6.4 Improvement in reference throughput with respect to the system from Figure

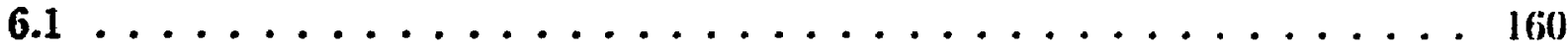

B.1 Suite CS3 and MR3 parameters (3 users and one server) . . . . . . 168

B.2 Suite CS4 and MR4 parameters (4 users and one server) . . . . . . . . 16!5

B.3 Suite CS5 and MR5 parameters (5 users and one server) $\ldots \ldots \ldots \ldots$. . 170

B.4 Exact and approx. results for test suite CS3 (mean error 0.46\%) . . . . . 173)

B.5 Exact and approx. results for test suite CS4 (mean error 0.53\%) . . . . . 175

B.6 Exact and approx. results for test suite CS5 (mean error 0.65\%) . . . . . 177

B.7 Exact and approx. results for test suite MR3 (mean error $0.38 \%$ ) _ . . . 182

B.8 Exact and approx. results for test suite MR4 (mean error 0.47\%) $\ldots \ldots \ldots 184$

B.9 Exact and approx. results for test suite MR5 (mean error $\mathbf{0 . 6 3 \%}$ ) $\ldots \ldots \ldots 186$ 


\section{List of Symbols and Terminology}

SRVN = Stochastic RendezVous Network

$\mathbf{R V}=$ RendezVous

QN $=$ Queueing Network

PN = Petri Net

GSPN = General Stochastic Petri Net

MC = Markov Chain

TDA $=$ Task-Directed Aggregation

TDA-1 $=$ TDA at vector state level

TDA-2 $=$ TDA at task state machine level

MVA $=$ Mean Value Analysis

TDA/MVA(1) = solution algorithm for a simple client/server SRVN whose clients send single-class requests to the server

TDA/MVA(2) = solut:ion algorithm for a simple client/server SRVN whose clients send multi-class requests to the server

TDA/DEC = iterative decomposition algorithm for complex SRVN models

"in RV" phase = execution phase of an accepting task, when the requester is blocked waiting for a reply from the acceptor (also named first phase in SRVN)

"post RV" phase = execution phase of an accepting task, executed in parallel with the requester (also named second phase in SRVN) 
$T_{i}=\operatorname{task} i$

$E(i)=$ entry set of $T_{i}$

$s_{i e p}=$ total execution time of task $i$, entry $e$ during phase $p$ (does not include the waiting for $\mathrm{RV}$ requests made in this phase)

$y_{(i e p)(j d)}=$ mean number of RV requests made by task $i$, entry $e$, phase $p$ to task $j$, entry $d$ $Y_{\text {iep }}=$ total number of $\mathrm{RV}$ requests made by task $i$, entry $e$ in phase $p$

$e_{i 0}=$ idle state of $T_{i}$

$u_{\text {iep }}=$ execution state of $T_{i}$

$q_{(i e p)(j d)}=$ queueing state of $T_{i}$

$r_{(i e p)(j d)}=$ "in $\mathrm{RV}^{\prime}$ state $\left(T_{i}\right.$ is served by $\left.T_{j}\right)$

$x_{i e p}=$ mean duration of phase $p$ of $T_{i}$, entry $e$

$w_{(i e p)(j d)}=$ queueing delay suffered by a RV request from $T_{i}$, entry $e$, phase $p$ to task $j$, entry $d$

$f_{i}=$ throughput of task $i$

$\varphi_{i e}=$ throughput of task $i$, entry $e$

$\sigma=$ state vector of a tasking system

$\sigma_{D}=$ subvector of designated task states

$\sigma_{N}=$ subvector of non-designated task states

$D=$ set of designated tasks

$\mathcal{S}_{i}=$ state set of task $i$

$\zeta_{i i}=$ state subset of task $i$

$\sigma_{i}=$ state variable for task $i$ (component of $\sigma$ )

$\sigma_{i}^{\#}=$ aggregated state of task $i$ obtained by TDA-2 
$\alpha_{i}, \beta_{i}, \tau_{i}=$ actual states of task $i$ (values of $\sigma_{i}$ )

$\Omega_{\alpha}, \omega_{\alpha}, \Gamma_{\alpha}=$ group of MC state lumped together by aggregation

$\mu_{i}=$ rate of the exponentially distributed duration of a state $\alpha_{i}$

$\lambda_{\alpha \beta}=$ aggregated transition rate from $\Omega_{\alpha}$ to $\Omega_{\beta}$

$\pi_{\alpha \beta}=$ probability to move from from state $\alpha_{i}$ to state $\beta_{i}$

$a_{i s}, q_{i s}^{(m)}, r_{i s}=$ states of client $T_{i}$ sending single-class requests to server $T_{s}$ in a simple clientserver SRVN

$\xi_{i s}=$ rate of the exponentially distributed duration of state $a_{i s}$

$e_{s 0}, \ldots, e_{s k p}=$ states of server $T_{s}$ in in a simple client-server SRVN, where the clients make one request class

$\mu_{s k p}=$ rate of the exponentially distributed duration of state $e_{s k p}$ of server $T_{s}$

$\delta_{s k p}^{Q}=$ short hand notation for a vector state $\sigma$, where the server executes entry $k$, phase $p$, and $Q$ is an ordered set of task indices containing the clients that are queued at the server in order of their arrival

$\mathcal{G}_{s k}=$ group of $\mathrm{MC}$ states where $T$, is serving $T_{k}$

$\mathcal{G}_{s k p}=$ group of $\mathrm{MC}$ states where $T_{s}$ is serving $T_{k}$ in phase $p$ (subset of $\mathcal{G}_{s k}$ )

$L_{k}=$ size of group $\mathcal{G}_{s k}$

$N_{p}=$ size of group $\mathcal{G}_{s k p}$

$\mathbf{Q}(n)=$ transition rate matrix of the MC for a simple client/server model with $n$ clients

$\mathbf{G}_{\mathbf{i i}}=$ diagonal submatrix of $\mathbf{Q}(n)$

$\mathbf{H}_{\mathbf{i j}}=$ non-diagonal submatrix of $\mathbf{Q}(n)$

$\mathcal{G}_{s k}^{\prime}=$ group of MC aggregated states obtained by TDA for $D=\{i, s\}$

$L_{k}^{\prime}=$ size of group $\mathcal{G}_{s k}^{\prime}$

$\mathbf{Q}^{\prime}(n)=$ aggregated transition rate matrix obtained from $\mathbf{Q}(n)$ by $\operatorname{TDA}($ where $D=\{i, s\}$ ) 
$\mathbf{G}_{\mathbf{i i}}^{\prime}=$ diagonal submatrix of $\mathbf{Q}^{\prime}(n)$

$\mathbf{H}_{\mathbf{i j}}^{\prime}=$ non-diagonal submatrix of $\mathbf{Q}^{\prime}(n)$

$\mathcal{G}_{s k}^{\prime \prime}=$ group of MC aggregated states obtained by TDA for $D=\{i, j, s\}$

$L_{k}^{\prime \prime}=\operatorname{size}$ of group $\mathcal{G}_{s k}^{\prime \prime}$

$\mathbf{Q}^{\prime \prime}(n)=$ aggregated transition rate matrix obtained from $\mathbf{Q}(n)$ by $\operatorname{TDA}$ (where $D=\{i, j, s\}$ )

$\mathrm{G}_{\mathrm{ii}}^{\prime \prime}=$ diagonal submatrix of $\mathbf{Q}^{\prime \prime}(\boldsymbol{n})$

$\mathbf{H}_{\mathbf{i j}}^{\prime \prime}=$ non-diagonal submatrix of $\mathbf{Q}^{\prime \prime}(n)$

$F_{i s}=$ throughput of RV requests from $T_{i}$ to $T_{s}$

$w_{i s}=$ mean queueing delay for a $\mathrm{RV}$ request from $T_{i}$ to $T_{s}$ (without service) in a simple client/server SRVN whose clients make single-class requests

$\mathcal{P}$ (vector) $=$ probability of the $\mathrm{MC}$ state indicated by vector

$\Phi($ vector $)=$ total input (output) flow of the MC state indicated by vector

$\phi_{g h}=$ transition flow from MC state $g$ to state $h$ (computed as transition_rate $\times$ state_probabilit $y$ )

Arrival instant probabilities for clients with single-class requests :

$A_{i s}^{j p}=$ probability that a $\mathrm{RV}$ request from $T_{i}$ arriving to $T_{s}$ finds the server busy in phase $p$ serving a request from another client $T_{j}$

$B_{i, j}=$ probability that a RV request from $T_{i}$ arriving to $T_{s}$ finds $T_{j}$ already in the queue (not in service).

$C_{i s j}=$ probability that a RV request from $T_{i}$ arriving to $T$, does not see $T_{j}$ either in queue or in service.

$B_{i s j}^{k p}=$ probability that a $\mathrm{RV}$ request from $T_{i}$ arriving when $T_{s}$ is executing entry $k$, phase $p$, finds $T_{j}$ in the queue

$C_{i s j}^{k p}=$ the probability that a RV request from $T_{i}$ arriving when $T_{s}$ is executing antry

$k$, phase $p$, does not see $T_{j}$ either in queue or in service

$\mathcal{R}_{i,}=$ set of all request classes from a task $T_{i}$ to a task $T_{s}$ 
$\mathcal{I}_{s}=$ set of all request classes arriving to server $T_{s}$

$\pi_{c}=$ probability that $T_{i}$ sends a class $c$ request to the server

$q_{c}, r_{c}=$ queueing and service states for different request classes of client $T_{i}$ with multi-class requests $\left(c \in \mathcal{R}_{i s}\right)$ in a simple client/server SIRVN server, where $c \in \mathcal{R}_{i s}$

$e_{c p}=$ state of server $T$, when serving class $c$ requests in phase $p$

$\mu_{c p}=$ rate of the exponentially distributed duracion of state $e_{c p}$ of server $T$,

$f_{c}=$ request frequency of a request class $c \in \mathcal{R}_{i s}$

$\xi_{c}=$ rate of a request class $c \in \mathcal{R}_{i s}$

$w_{c s}=$ queueing delay of a request class $c \in \mathcal{R}_{i s}$

$\mathcal{G}_{c s}=$ group of $\mathrm{MC}$ states where $T$, is serving class $c$ requests

Arrival instant probabilities for clients with multi-class requests :

$A_{a}^{b p}=$ probability that a class $a$ request arriving to $T_{s}$ finds the server busy in phase $p$, serving a class $b$ request

$A_{a}^{0}=$ probability that a class $a$ request finds the server idle

$B_{a b}=$ probability that a class $a \mathrm{RV}$ request arriving to $T_{\mathbf{s}}$ finds a class $b$ request waiting in queue (not in service)

$C_{a j}=$ probability that a class $a$ request from $T_{i}$ arriving to $T$, does not see any request from $T_{j}$ in queue or in service

$B_{a b}^{c p}=$ probability that a class $a$ request arriving to $T_{a}$ when $T$, is serving $c$ in phase $p$, finds a class $b$ request waiting in queue (not in service)

$C_{a j}^{c p}=$ probability that a class $a$ request from $T_{i}$ arriving when $T_{s}$ is serving $c$ in phase $p$ does not see any request from $T_{j}$ in queue or in service 


\section{Chapter 1}

\section{Introduction}

\subsection{Research Motivation}

The performance properties of a computer system are important throughout its whole life: cycle, playing a key role in the process of design, development, configuration, tuning, operation and maintenance. While the concept of performance is understood by most people. as being related to the notions of speed and efficiency, a more formal definition requires the following steps [Fer86]:

a) a precise definition of system performance and of the performance measures that are expressing it;

b) methodologies for performance evaluation, through which quantitative values are assigned to the performance measures of a given system.

A list of typical performance measures for computer systems is: throughput, capacity, response time, utilization, speedup, reliability, availability, etc. Due to the diversity of computer systems and of their applications, there is no unique and generally accepted definition for system performance. The most appropriate performance measures may be different from one type of system to another. For example, the performance of a real time system is better described by the measure response time, while the performance of a batch processing system is better described by throughput. The quantitative values of performance measures depend on the workload under which the system is functioning while the performance is evalutated. The workload has to be characterized by another series of measures on the systrm input.

Performance evaluation methods are divided into two main categories: 
- measurements on existing systems, that are implemented, instrumented and running

- modelling systems in any phase of development, and analysing the model.

This thesis deals with models. A model is a logical or functional representation that mimics the system under study. The modeller can analyse the behaviour of the model in different conditions, and infer the behaviour of the actual system under similar circumstances. The construction of a model implies usually a considerable process of abstraction, for the model should be simpler and easier to analyse then the actual system. However, it is very difficult to decide which features are relevant and should be represented in the model, and which are not. Thus, the construction of a performance model involves often decisions that are not based on a rigorous scientific foundation, but rely mostly on the ability, ingenuity, and experience of the modeller.

There are two main classes of performance models for computer systems: simulation and analytical models. While simulation provides mainly quantitative performance results by means of estimates for system operating points, analytical models provide not only quantitative information, but also a qualitative understanding of the relationship between system parameters and performance measures. Thus, the analyst gains insight into aspects that affect system performance by analytical modelling. Another advantage is that the cost of solving analytical models is generally much lower than the cost of simulation. The main disadvantage of analytical models is the fact that their accuracy is usually lower than that of simulation models. This is due to modelling restrictions imposed in order to insure the existence and feasibility of analytical solutions. Firstly, analytical models may be based on assumptions that simplify mathematical analysis, but do not correspond to the reality. Secondly, the size of analytical models is often limited by the exponential growth of solution complexity, a factor which does not apply to simulation models. This thesis is particularly oriented toward 3 improved solution methods which are practical for large models, to make this second problem less severe. Although both simulation and analytical models play an important role in computer performance modelling, the present research is focused on analytical modelling only. 


\subsubsection{Software abstraction for performance modelling}

The components of a computer system are organized in layers: application software rumning on top of an operating system, implemented in turn on a hardware system. Each layer has its own contribution to the performance of the entire system, and must be represented in performance models in order to obtain acceptable accuracy.

The present research is focussed on the representation of software in performance mod els. For reasons that have been mentioned already, a performance model contains only an abstracted view of the actual system. There are many kinds of details associated with software, and thus it is possible to identify abstractions of many kinds, appropriate for diffrent purposes in different life cycle stages. The software abstractions for performance analysis are different from those used for software specification, design, implementation, ctc., For example, the semantics of software functions are irrelevant for performance evaluation; the. relevant information concerns their duration and order of execution, as well as the requirements for resources. Regarding the data structures, neither data values, nor modifications on data performed by different software modules are important in performance models. Data may be merely viewed as shared resources that must be accessed by competing software modules, or as tokens circulating from module to module that act as activation signals. However, even if the software abstractions for performance evaluation are different from the: abstractions for software development (specification, design, etc.), it is desirable to maintain a certain semantic equivalence between the former and the latter. This equivalence facilitates the derivation and calibration of performance models, as well as the interpretation and use: of their results for further improvements of the actual software.

The need for performance models in : hich the software is represented as close as possil, le to its design description becomes even more important if we consider the idea (advocated by [Smi90] among others), that performance analysis should support the software development. throughout all its life cycle stages. It should begin early by assessing requirements and different design alternatives, while the widest range of options exists, and continue throughom! the life cycle to implement systems that meet their performance objectives. Even if the parameters for software performance models are only approximate in early life cycle phases. it is still worthwhile conducting the assessments, since performance problems in the airly 


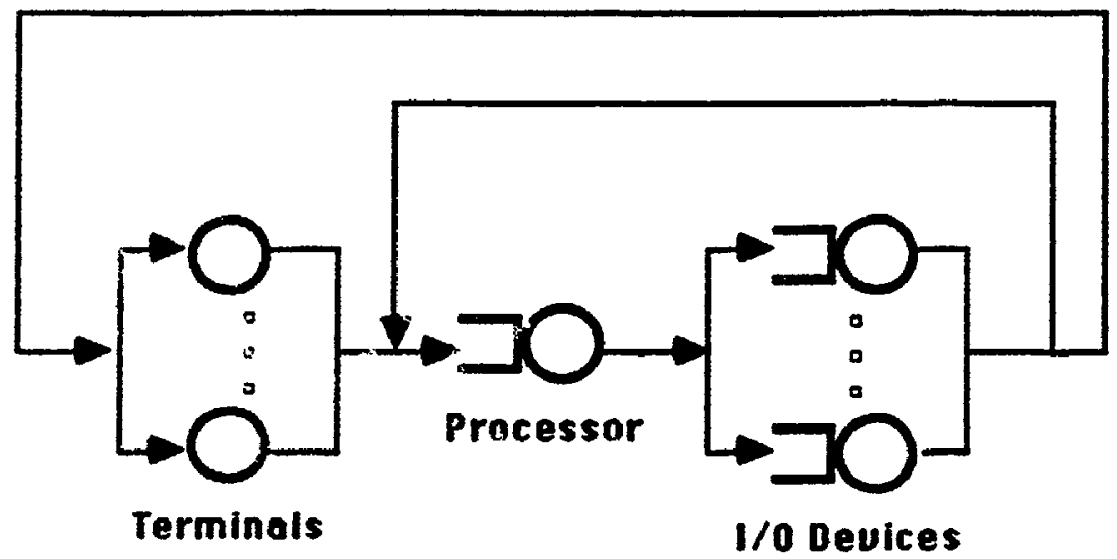

Figure 1.1: QN model of a time sharing system

stages tend to have order-of-magnitude effects on final performance metrics. Thus, the software product performance can be orders of magnitude better if the problems are detected and corrected early, rather than leaving them for late code-tuning.

The research in the field of analytical computer performance modelling began in the 1960 s, when queueing network formalism started to be used to model general purpose multiuser computer systems, i.e. multiprogramming and time sharing systems. The performance of such systems is strongly affected by the queueing delays encountered by multiple jobs attempting to access and receive service from shared resources (mainly hardware devices). These resources were modelled as service centers, and the software jobs as customers moving from one center to another, waiting in a queue if the required resource was busy, and then receiving a certain amount of service. (An example of a typical QN model for a time sharing system is shown in Figure 1.1). A more detailed discussion of queueing network models follows in sections 1.1.3 and 2.1. The queueing network abstraction was quite satisfactory to model "batch" or "interactive" jobs consisting of independent sequential programs that competed for shared resources, but did not communicate or synchronize directly with each other.

The developments of the last decade in the field of computer systems, and especially of distributed systems, brought significant changes in software structure and behaviour. Many software systems are now inherently concurrent and nondeterministic, running on systems where the control is decentralized and data are distributed. The concurrent programs consist 


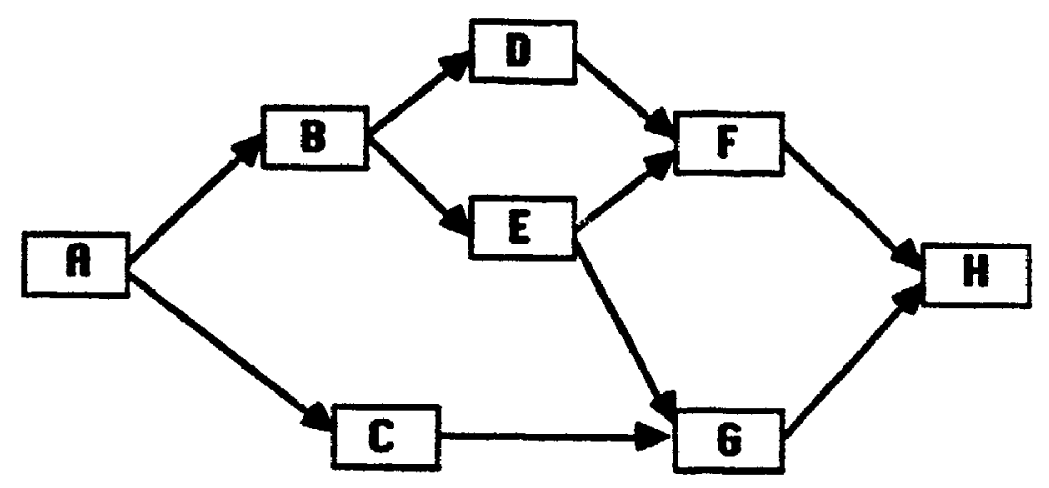

Figure 1.2: Flow model for concurrent software

of a set of parallel threads of execution (tasks or processes) that not only compete for shared resources, but also manifest a strong functional interdependence by cooperating, conmunicating, and synchronizing with each other. Examples of such systems are: distributed datit bases, real time control systems, communication switching systems, and signal proressing systems. The abstract representation of software jobs as independent customers competing for shared resources worked well for models of sequential programs running on time sharing systems, but is not as satisfactory for concurrent software running on distributed systems. Other representations are necessary to emphasize not only the contention for resources, but also the functional interdependence between parallel threads of execution insicle. the concurrent software. Two main classes of abstractions representing parallel software for performance evaluation (used in either simulation or analytical models) can be found in the literature:

a) Flow models for performance analysis are derived from the well known control flow or data flow models used for software specifications, design, logic verification, etc. 'The. performance flow models resemble critical path or PERT charts, showing the securcut ial and parallel execution constraints in the software. A flow model is described by a graph (see Figure 1.2 for an example). A node represents a software entity namiril an activity, that usually is a sequence of executable code with a beginning and an end, executed in a single threaded manner. The graph shows the precedence relinticus between activities, and also the fork/join of the execution flow(s) in branches. Flew 
models have the advantage of revealing potential parallelism in software and critical paths. For a complete performance analysis it is necessary to know also the set of common resources shared by activities and the workload requirements There are at least two versions of flow models used for analytical performance analysis:

a1) In the first version, an activity may only communicate with its predecessor(s) at the beginning of its execution, and with its successor(s) at the end. The activities that are situated on parallel branches can compete for shared resources, but do not communicate or synchronize with each other. This type of representation was used for example in [TB85, HT83].

a2) In the second version, the parallel activities may communicate and synchronize at any point of their execution, as well as compete for shared resources. This version is a more complex model of the software, and has been used in [PS87] for the analysis of a real-time control sysiem.

b) Set of parallel communicating processes (tasks) is a well known paradigm for concurrent software used for example in the specification languages CCS [Mil80], CSP [Hoa85], and PAISLey [ZS86]. A task or process represents an entity that is executed in a single threaded manner, and has a cyclical behaviour. The concurrent tasks intercommunicate by means of messages. The communication may be synchronous (send and wait for reply), or asynchronous (send without waiting for reply). Such a tasking system may be conveniently described by a set of communicating finite state machines. A complete performance model must also specify the set of common resources shared by tasks, and the workload requirements. This type of model was used for analytical performance evaluation, for example, in [Woo89], [Rol88] and [Rad84].

\subsubsection{Software servers and client/server systems}

The basic building blocks representing software entities (activities, tasks, or processes) in both classes (a) and (b) of abstractions discussed in the previous section exhibit a common characteristic: a software entity is activated by a request (signal or message) that may arrive when the entity is idle or busy with a previous request; while executing (or serving the 
request), the software entity may require other resources (hardware or software) for which it queues and waits to be served.

This leads to the notion of a "software server", that can accept requests from different clients and serve them according to its scheduling policy. Remote Procedure Calls are a widely used example of software servers, usually supported by distributed operating systems (an example is Mach [Ras87]). Software servers have several basic characteristics which make them different from hardware servers, and more difficult to model:

- A software server plays a dual role: while serving its own clients (that are blocked for service), it also acts as a client with respect to other servers (hardware or software). Thus, the service from a soltware server may include several layers of nested services. In consequence, the software server may be perceived as "busy" by its own clients when, in fact, it is blocked, waiting for some other resource(s). This may have a strong impact on system performance, especially when a software server is the bottlencrk in the system and none of the hardware resources are used at full capacity. (This problem will be addressed in section 6.3).

- A software server may have a "post-service" phase that is executed in parallel with the client. This phase can model activities that are necessary to complete the service, but can be done without having the client blocked for their duration. For example, a software server managing a file system may receive a write request from a client process, that will be blocked only for a short time, until the command and the data have been accepted by the server, but will be able to continue its execution in parallel with the server when the actual I/O operation is performed. The "post-service" phases increase the internal concurrency of the system.

- A software server may offer a range of services with different workload requirements (iem i.e. multi-class service, and each client will require several of this services. For example, an user of a data-base may send requests of different types (search, update. delete) to the server task that manages the access to the data-base.

- There are some restrictions on the type of scheduling policy for a soitware server, dire: to the necessity to preserve data integrity. Thus, "round-robin", "processor-sharing", 
or preemptive policies that are much used for CPU scheduling, are not appropriate for software servers.

These characteristics of software servers have, usually, a strong impact on system performance, and must be captured in a performance model for concurrent software. Existing performance models do not handle all these characteristics together. The analytical model Stnchastic Rendezvous Network (SRVN) that is the object of this research is however able to capture all of the previous characteristics. SRVN will be discussed informally in section 1.1.4 and formally in section 3.1 .

\subsubsection{Comparison of main analytical modelling methods}

Two of the best known analytical modelling methods used for computer performance evaluation are queueing networks (QNs) and Petri nets (PNs). The present section compares briefly their modelling capabilities, advantages and disadvantages, emphasizing the way the concurrent software is represented. Chapter 2 gives a more detailed survey of the background literature for analytical performance models, discussing not only their modelling capabilities, but also their solution methods.

\section{Queueing Networks}

Queueing Networks (QNs) are well established as practical tools in computer system performance analysis and capacity planning ( see, for example, [LzGS84], one of many books in the field). A QN model is a collection of service centers or nodes (each with its own queue and servers), and customers or jobs (representing the consumers of service). Every service center represents a common resource, shared by customers, and has an established discipline to dispatch the requests for service. After the completion of service at one service center, a customer may move to another service center according to a routing chain. Except for contention for common resources, the customers are independent. They may be divided into several classes, such that all customers within a class are statistically identical with respect to the routing probabilities and service demands. A class may be closed, if the customers cannot arrive from outside nor leave the system, or open, if arrivals and departures are allowed. 
The incontestable advantage of QNs is their combination of expressive power and solution efficiency. The notation used to describe the models is powerful, and at a high level of abstraction; the models are easy to build, and require relativel: few parameters. Furthermore. there exist a large range of solution methods, some of them very efficient (especially those for the class of product form QNs). Extensive empirical experience gained with the use of QN models shows that the results are sufficiently accurate for the applications in which they are typical employed [LZGS84].

QNs have been extensively used for analytical computer performance modelling; an c $x$. cellent survey with an emphasis on development of practical importance is given in [Lav89]. In a "classical" QN model for computer performance, the service centers usually represent. hardware resources, while the customers represent software jobs. This is a very appropriate model for time sharing systems where the jobs compete for shared resources but do not communicate or synchronize directly. However, QNs have important limitations in modelling parallel software for distributed systems; they are unable to represent in a direct way the. synchronization, and fail to capture the duality "server/client" of software entities. Ad hor extensions and approximate solution methods have been proposed to overcome some of these shortcomings (as surveyed in chapter 2), but they are not general or straightforward enough.

\section{Petri Nets}

The second class of models used for performance analysis of concurrent systems are the Petri Nets (PNs) with timing properties. They represent a relatively new approach for performance evaluation, being extensions of classical PNs that were initially introduced as tools for analyzing the correctness of asynchronous parallel systems. The representation of synchronization in PN models is fundamental to the notation. Classical PNs consist of a set of places, a number of tokens that reside at the places, and a set of transitions that represent events causing tokens to move from one place to another. There are two 1 ypess of PNs with timing properties: "stochastic" PNs (isomorphic to continuous-time Markov chains [Mol82, AMBC84]), and "timed" PNs (isomorphic to discrete-time Markov chains [RP84, HV87]). A comparison of QNs and PNs is presented in [VZL87]. The big advantinge: of PNs is their modelling power. The representation of concurrent software can be dost at almost any level of detail, from a very coarse to a very fine grain. PNs are very appropriate 


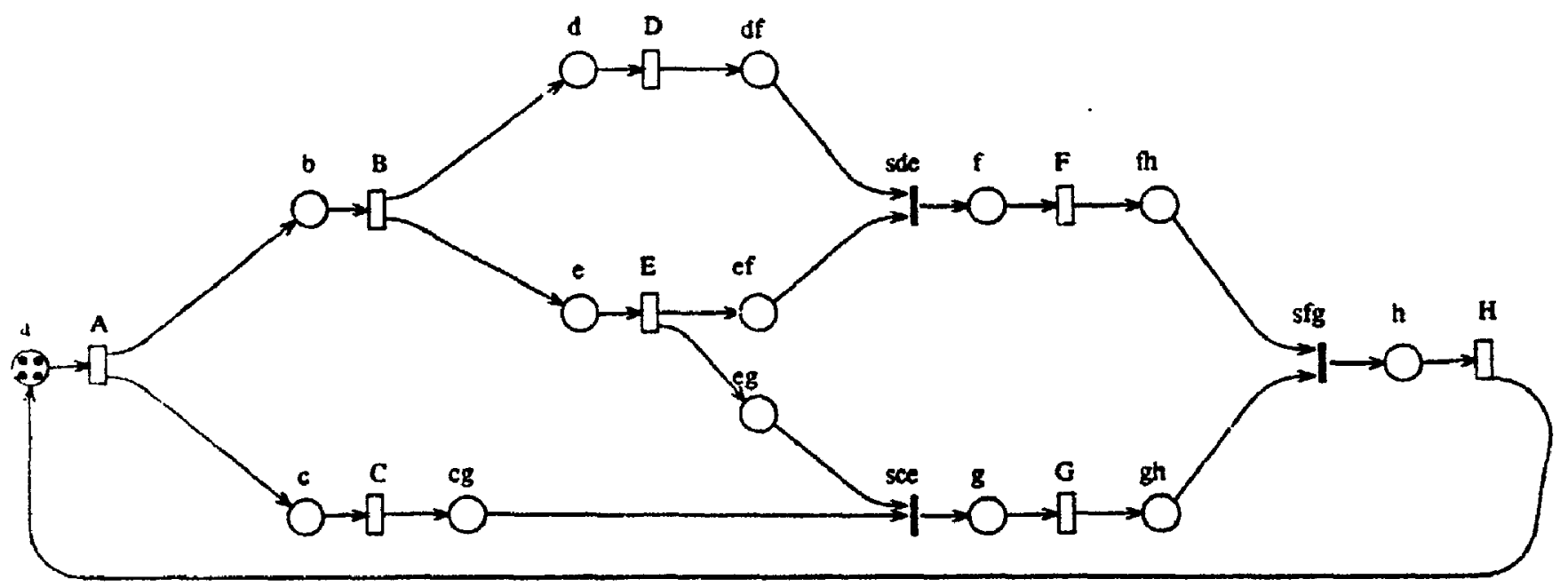

Figure 1.3: PN model for the flow graph model from Figure 1.2

to represent flow models of concurrent software. For example, Figure 1.3 shows the PN model of the flow model given in Figure 1.2, assuming that the various activities do not share common resources.

A main disadvantage of $\mathrm{PNs}$ is their poor ability to model contention for resources. The PN basic constructs (i.e., places, transitions, and tokens) have a low level of abstraction. Unlike QNs, PNs have no basic constructs to represent queues with different scheduling policies. Consequently, PN models are not as compact or easy to build as QN models. Another disadvantage of PNs is their lack of efficient approximate solution methods. The exact solution imply the construction and analysis of a Markov chain model, that suffers from exponential growth of the state space, and thus severely restricts the size of tractable models. Section 2.3 gives a more detailed survey of PN modelling capabilities and solution methods.

\subsubsection{Stochastic Rendezvous Networks}

The previous section shows that QNs and PNs, the most utilized methods for analytical performance analysis of computer systems, have complementary advantages and disadvantages in representing the concurrent software. It seems that a more appropriate modelling formalism for such systems should be situated on middle ground between QNs and PNs. 
Stochastic RendezVous Network (SRVN) is a new analytical model that tries to combine the advantages of both QNs and PNs for a specific class of systems at the price of losing modelling generality. SRVN was proposed in [Woo89] (a preliminary version in [Woo86]) to model concurrent systems with rendezvous (RV) style of communication.

Many languages and operating systems support intertask communication via different types of rendezvous, where the sending task is blocked until it gets either a signal that its message is accepted or a reply, and then continues its execution in parallel with the receiver. Examples include Ada [DOD83] and XMS [GC84] that use procedural request/accept constructs, and Occam [Hoa88], Thoth operating system [Che82], V-System [Che84] and Harmony operating system [Gen87] that use send-and-wait and receive-and-wait primitives. Furthermore, Remote Procedure Calls, usually supported by distributed operating systems (an example is Mach [Ras87]), can also be modelled as special cases of RV-based intercommunication with no post-RV execution of the receiver. The RV is useful because it combines synchronization and communication, it simplifies resource management (no need for buffering of messages by the kernel), and it facilitates the logical analysis of task behaviour. However, because both the sender and the receiver wait at certain points, a deadlock is possible. Also, the waiting may create performance problems, limiting the speed of the system.

SRVN models represent the concurrent software as a set of communicating tasks. A task accepting RV requests has a FIFO input queue, where the RV requests from other tasks wait their turn to be served, while the requester tasks are blocked. The acceptor task executes in a cycle that starts with a $R V$ accept. Following the RV communication rules, if its input queue is empty, the acceptor waits until a RV request arrives. After a request was received, the acceptor executes the so-called "in RV" phase, at the end of which the requester task will be released, resuming its work. The acceptor continues and executes a "post RV" phase. in parallel with the requester. Each phase is a sequence of identical stochastic execution periods (with known distribution) when the task uses a processor, separated by RV requu'sts to other tasks (randomly selected with known probabilities).

The class of SRVN considered in the thesis allows for different behaviours of a task depending on the entry point for which a request has arrived. An entry (the term is from Ada) may also be called a port or socket. The workload parameters characterizing the tasks (execution times and number of RV requests) are given by entry and phase. A formal 


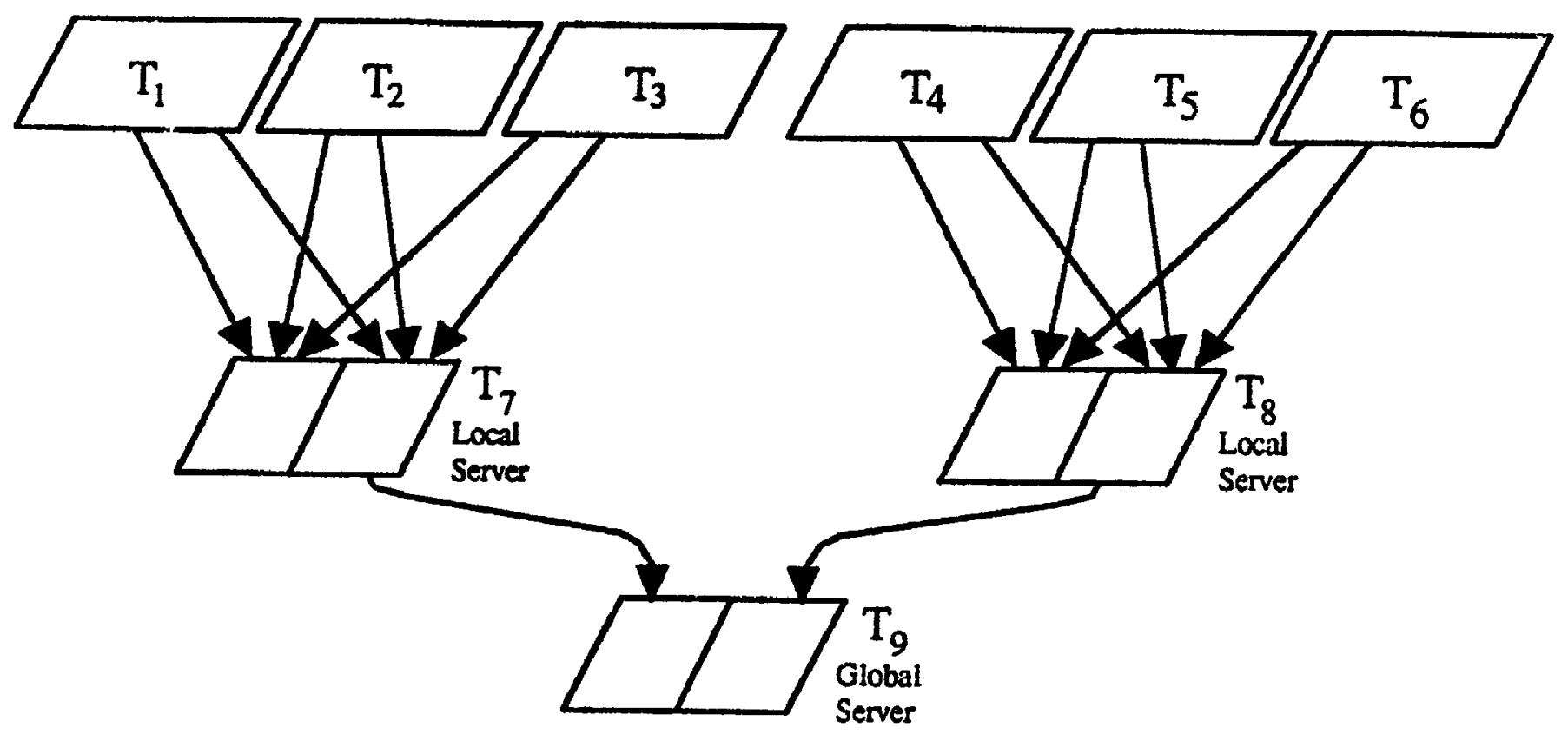

Figure 1.4: Example of an SRVN model

description of the model is given in section 3.1 .

An SRVN model is described by an acyclical communication graph that shows the pattern of interactions between tasks. The model is driven by a set of reference tasks, that do not accept RV requests, but rather cycle endlessly, alternating execution periods with RV with other tasks. The reference task throughputs represent the main performance measure for the model.

Figure 1.4 gives an example of an SRVN model representing a hierarchical database system. There are two local data servers, each with its own set of users, and a global data server receiving requests from the local servers only. The majority of user requests are served locally, and only a small fraction of requests (between $10 \%$ and $30 \%$ ) need also the global server. In order to model the two classes of user requests, each of the local servers has two entries: the first does not require any nested RVs, while the second sends RV requests to the global server. The global server also offers services with different workload for each of its clients.

Every server entry has an "in RV" phase (modelling the part of service in which the client is blocked waiting for a reply), and a "post RV" phase (modelling activities that 
can be executed in parallel with the client, such as writing files, sorting, or house-keeping operations).

A model with this tasking architecture can be used to represent different types of systems, for example:

- A software system implemented on a local area network, where several application processes (modelled as local servers) accept and serve requests from different users, sharing a database (or file) server (modelled as a global server). The application processes are running on different processors, and are provided with large $1 / O$ buffers in order to insure that a reasonable part of user requests are served locally without accessing the global server.

- A geographical distributed database, where most of the queries can be solved by a local database server, while the rest need global information stored into a remote central server.

The SRVN model is able to capture the following features of the system: nested service offered by the the hierarchy of servers, change of request classes by the users, and parallel execution between users and servers (second phases). (The SRVN results for this type of model will be presented in section 6.2).

In the basic SRVN proposed in [Woo89], each task offers its own parallel service as if it had its own processor. Extensions to the basic SRVN consider the following aspects: processor contention in [MWNP88], task priorities in [MWNP89], tasks with different entries in [WNPM89], pipelines of tasks in [WNM+88]. The author of the thesis was a member of the team that developed, implemented, and assessed these SRVN extensions.

It is important to remark that the RV mechanism provides a natural way to include the contention for hardware resources in SRVN models; such type of resources may be represented as tasks having only "in $\mathrm{RV}^{\text {" phase. }}$

Among the strength of SRVN are the ability to represent the synchronization implied by the RV mechanism, as well as the duality "server/client" of software tasks. The level of abstraction and compactness of SRVN models is comparable with that of QN models. The SRVN solution methods are approximations based on concepts similar to the Mean Value Analysis framework for QNs, having similar efficiency. The SRVN models have the: 
disadvantage that they still do not capture some relevant features of parallel software, such as fork/join of execution flows. However, they are adequate for most client-server systems.

\subsection{Research Goals and Contributions}

\subsubsection{Long and Short Term Goals}

The long term goals of this research are motivated by the general need for better analytical performance models for concurrent software system, able to capture relevant features that have a strong impact on the overall performance as, for example, those discussed in section 1.1.2. The problem of software modelling becomes even more important when we consider the idea that performance analysis should support the software development process throughout all its life cycle stages. The estimation of performance should begin early by assessing requirements and different design alternatives, while the widest range of options exists, and continue throughout the life cycle to implement systems that meet their performance objectives.

In view of this intended application, the software performance models should meet the following requirements:

- To capture the main features of the concurrent software with a strong impact on overall performance ;

- To represent the software as close as possible to its design description, in order to facilitate the derivation and calibration of performance models, as well as the interpretation and use of their results for further improvements;

- To be solvable for models of practical size, which means that the solution methods should avoid (or make less severe) the exponential growth of computational complexity.

The short term goals of the thesis are related to the improvement of the solution methods for SRVN models of software with rendezvous communication, being motivated by two main factors:

- The need for a more rigorous theoretical foundation to replace the ad hoc collection of heuristic approximations used in the previous SRVN solutions; 
- The search for SRVN solution methods with a better accuracy. The last requirenent listed under long term goals points to approximate solution methods based on model decomposition. Given that the intended application is performance prediction in early software life cycle stages, when the software is not built yet, and the parameters for performance models are only approximate (from previous experience with similar sys. tems or from prototyping), our accuracy goal is an average relative throughiput error under $5 \%$, and a maximum error under $10 \%$.

\subsubsection{Contributions}

The research contributions of the thesis are the following:

- Definition of Markov Chain "task-directed aggregation"(TDA) as the theoretical foundation for the algorithms proposed in the thesis. There are two types of TDA: TDA-1 aggregates across tasks, so the identity of non-designated tasks disappears in the new state vector, while TDA-2 aggregates states within tasks.

- Study of TDA-1 and TDA-2 properties, by proving three lemmas.

- Development of an approach based on TDA for the development of an iterative decomposition algorithm for a tasking system with synchronous communication, dealing specifically with the problem of obtaining submodel parameters from initial parameters and from imported results of other submodels.

- Study of the Markov Chain (MC) for a simple client/server SRVN with a server and any number of clients $n$, giving its balance equations and showing that its size grows exponentially with the number of clients $n$. (Each client requests one entry of the server and gets a different service).

- Application of TDA to the MC of a simple client/server SRVN, proving two theorenss that show that the size of the aggregated MCs grows linearly with the number of clients $n$, and that the transition rate submatrices of the aggregated MCs have the same form for any number of clients $n$. 
- Derivation of the equations for arrival instant probabilities from the balance equations of a set of aggregated MCs obtained by TDA.

- Development of the algorithm TDA/MVA(1) for a simple client/server SRVN, based on the MVA framework and using the arrival instant probabilities equations derived previously. Assessment of TDA/MVA(1) accuracy and convergence through experimental results.

- Extension of TDA/MVA(1) for the case where the clients are allowed to request several server entries (i.e. the clients can change the requests class), that implies the derivation of a new set of arrival instant probabilities and the introduction of an extended algorithm TDA/MVA(2). The need for this extension arises from actual features of the software servers. Assessment of the accuracy and convergence of TDA/MVA(2) is done through experimental results.

- Development of an iterative decomposition algorithm TDA/DEC for complex SRVN models with any number of servers and multi-layered service. Application of the algorithm to an SRVN model of an hierarchical database, and to the study of an example of software bottleneck.

\subsection{Thesis Overview}

Chapter 2 gives a review of the literature in the field of analytical performance models relevant to software modelling in general, and to SRVN models in particular. The two main modelling techniques, QNs and PNs, are studied from two viewpoints: the ability to capture important features of the concurrent software, and the efficiency of their solution methods. Also, a section was devoted to solution techniques at the Markov chain level, that can be applied to analytical models that are isomorphic to a Markov chain. The bibliographical review provides a conceptual framework for the new solution method for SRVN models. Therefore, a summary of some major features of QN models will be given here.

Chapter 3 has two parts. The first gives the formal definition and the notation for an SRVN model, and reviews the principle of the existing heuristic MVA solution algorithm for SRVN. The second part defines and studies the properties of TDA-1 and TDA-2, then 
introduces an approach based on TDA for the development of an iterative decomposition algorithm for a tasking system with synchronous communication, dealing specifically with the problem of obtaining the parameters for a submodel from results imported from other submodels, and from initial parameters.

Chapter 4 studies the Markov Chain model of a simple client/server SRVN, applies TDA to this MC proving that the size of the aggregated MC grows linearly with the number of clients $\boldsymbol{n}$ and that the diagonal transition rate submatrices of the aggregated MCs have the same form for any number of clients $n$, derives the equations for arrival instant probabilities, develops the algorithm TDA/MVA(1) and assesses its accuracy and convergence through experimental results.

Chapter 5 gives the derivation of a new set of arrival instant probabilities and introduces an extended algorithm TDA/MVA(2) for the case of a simple client/server SRVN where the clients are allowed to request several server entries (i.e. the clients can change the requests class). Assessment of the accuracy and convergence of TDA/MVA(2) is done through experimental results.

Chapter 6 presents a new iterative decomposition algorithm TDA/DEC for complex SRVN models with any number of servers and multi-layered service. The algorithm is then applied to an SRVN model of an hierarchical database, and for the study of an example of software bottleneck.

Chapter 7 presents the conclusions and directions for future research. 


\section{Chapter 2}

\section{Literature review}

\subsection{Queueing Network models and solution methods}

Up to the present, the most widely used analytical modelling techniques for performance models of computer systems have been the queueing networks (QNs). Although not directly used in the present research, the QNs provide a conceptual framework for the approximate SRVN solution method proposed in this thesis that is based on model decomposition and iterative solution, and is taking an approach similar to the Mean Value Analysis principle for the solution of a submodel.

As described in section 1.1.3, a QN consists of a collection of service centers (or nodes), each with its own queue and servers, and customers, that move from one service center to the other, waiting for and receiving service. The customers may be divided into several classes, such that all customers within a class are statistically identical. A class may be closed, if the customers cannot arrive from outside nor leave the system, or open, if arrivals and departures are allowed.

An QN with $n$ queues can be modelled as a stochastic process whose states are defined as a collection $\left(s_{1}, s_{2}, \ldots, s_{n}\right)$ of the states of each individual queue in the system. This stochastic process is Markovian if every service center in the network has a service distribution with rational Laplace transform (see, eg. [Mol89] p. 204).

The direct solution of a Markovian QN implies finding and solving the linear set of global balance equations, in order to obtain the steady-state probability distribution that represents a complete performance measure of the systern. From the steady-state probability distribution we can obtain any other performance measure, as utilizations, throughputs, 
response times, sojourn times, etc. Unfortunately, the direct solution is difficult or even impossible to be used for the majority of practical problems, due to a very rapid growth in the number of states and consequently in the linear system size for a small increase in model complexity (problem known as state explosion). Even for simple models, the state space may be very large, and the direct solution computationally intense [Ste78]. For bigger models, the direct solution becomes intractable.

\subsubsection{QNs with product form solution}

There is a subset of Markovian QNs with special properties that can be solved by fast conputational algorithms, avoiding the direct solution; they are called separable QNs or product form QNs. Their solution has the important property, discovered by Jackson [Jac:57, Jac63], that the steady-state probability distribution for the entire system is obtained from the product of the steady-state probability distributions for the individual queues. More formally, the joint probability density function of the number of customers in each queue in equilibrium state is the product of the marginal probability density functions.

The product form property for systems with a single class of customers may be expressed as follows [SC81]:

$$
P\left(n_{1}, n_{2}, \ldots, n_{M}\right)=\frac{X_{1}\left(n_{1}\right) X_{2}\left(n_{2}\right) \ldots X_{M}\left(n_{M}\right)}{G}
$$

where:

$P\left(n_{1}, n_{2}, \ldots, n_{M}\right)$ is the steady-state probability of a network state in a network with $M$ queue, $n_{i}$ being the number of customers in queue $i$.

$X_{m}\left(n_{m}\right)$ is a factor obtained from the marginal queue length distribution of queue $m$ in isolation.

$G$ is a normalizing constant that forces the sum of network state probabilities to 1 .

The product form QNs have been extensively studied. Jackson's result has been extended to encompa $s$ networks with heterogeneous classes of customers, with both closed and open routing chains, with various queueing disciplines, with general service time distributions for some of these disciplines, and with some state-dependent behaviour. (The customers of a 
class have a identical stochastic behaviour and workload requirements). Baskett, Chandy, Muntz, and Palacios have established in [BCMP75] the following set of assumptions for a basic group of networks with product form solution:

1. Scheduling disciplines allowed:

- FIFO:First In First Out

- PS:Processor Sharing

- LCFS-PR:Last Come First Served Preemptive Resume

- IS:Infinite Server or delay service

2. Service time distribution:

- The service time at an FIFO server should be exponentially distributed with the same mean for all customer classes;

- The service time at a PS, LCFS-PR, and IS server can have any distribution with rational Laplace transform (in practice, this means a general distribution, since almost every distribution can be approximated this way). The mean service time for different classes may also be different.

3. State dependent service rates:

- The service rate at a FIFO server can depend only on the total queue length of that server;

- The service rate for a class at PS, LCFS-PR and IS servers can depend on the queue length for that class, but not on the queue length of other classes;

- The overall service rate of a subnetwork can depend on the total number of customers in the subnetwork.

4. Arrival Process: In open networks, the arrival process should be Poissson (no bulk arrivals are permitted).

5. Routing: each class has its own Markovian routing specifications for transitions from one queue to another. It is also possible to change class, which gives rise to group of classes called chains; this possibility will be ignored here. 
These assumption lead to the property of "local balance" in product form QNs [CHTT7], i.e., the flow rate into a state due to an arrival of a class $r$ customer at a center $j$ equals the flow rate out of that state due to a departure of a class $r$ customer from the same center.

\subsubsection{Exact computational methods for product form QNs}

\section{Convolution and Mean Value Analysis algorithms}

The advantage of product form solution is the existence of fast computational algorithms that avoid the use of the direct method. Two major algorithm which exploit in different ways the recursive structure of the product form solution are the Convolution algorithin and the Mean Value Analysis algorithm.

The Convolution algorithm was first proposed by Buzen in [Buz71] for exponential networks, and has been extended since then for all kinds of product form QNs [SC81, Sau83]. From historical point of view, the reporting of the convolution algorithm marked a turning point in the field of queueing network research. Though the concept of product form solution has been known since late fifties, the QN modelling applications did not really take off before fast solution methods were available. The convolution algorithm computes the normalization constant $G(N)$ by using some recurrence relations in function of queue populations, and then determines performance measures as probability distributions, utilizations, throughputs, etc., in function of $G(N)$. There is a problem in the implementation of the convolution method, due to the fact that relative utilizations are used during the computation instead of actual utilizations. This can cause a numerical overflow or underflow, since the relative values may be either very large or very small. A way of dealing with this problem is to dynamically rescale the relative utilizations as the computation progresses.

Another algorithm that never computes the normalizing constant, but simply uses the averages values of different performance measures is the Mean Value Analysis (MVA). MVA, proposed by Reiser and Lavenberg in [RL80], is $\mathrm{r}$ ed on two fundamental theorems. First, it uses Little's result applied to each queue in the network and to the network as a whole. Second, it uses the arrival theorem formulated by Lavenberg and Reiser [Lav80], which statess that the stationary state probabilities at instants at which customers of a particular class arrive at a particular service center are equal to the stationary probabilities at arthitrary 
times for the network with one less customer of that class.

The MVA equations for a product form network consisting of only single server fixed rate (SSFR) and infinite server(IS) service center are given below for reference. The following notations are used:

$J:$ the number of service centers in the network

$K:$ the number of chains

$N_{k}:$ the population of class $k$

$\mathrm{N}=\left(N_{1}, \ldots, N_{K}\right):$ the network state vector

$e_{k}: a$-dimensional vector with the $k$-th element equal to one and the rest of elements zero

$S_{k j}$ :the mean service time for a class $k$ customer at center $j$

$\theta_{k j}:$ the visit ratio for a class $k$ customer at center $j$ (scaled to be unity at a designated center visited by class $k$ )

$L_{k j}(\mathrm{~N}):$ the mean queue size for class $k$, center $j$ (including the customer in service)

$W_{k j}(N)$ : the mean waiting time for class $k$ at center $j$ per visit (including the service time)

$\lambda_{k}(\mathbf{N})$ :the class $k$ throughput (at the designated center for the class).

The MVA equations for $k=1, \ldots, K$ are:

$$
\begin{gathered}
\lambda_{k}(\mathbf{N})=N_{k} / \sum_{j=1}^{J} \theta_{k j} W_{k j}(\mathbf{N}) \\
L_{k j}(\mathbf{N})=\lambda_{k}(\mathbf{N}) \theta_{k j} W_{k j}(\mathbf{N}) \quad j=1, \ldots, J \\
W_{k j}(\mathbf{N})= \begin{cases}S_{k j}\left[1+\sum_{l=1}^{K} L_{l j}\left(\mathbf{N}-\mathbf{e}_{k}\right)\right] & \text { if center } j \text { is SSFR } \\
S_{k j} & \text { if center } j \text { is IS }\end{cases}
\end{gathered}
$$

These equations express an exact relationship between the performance parameters and can be used recursively for an exact solution of the network. Besides the advantage of totally avoiding the problem of overflow or underflow, MVA has also the advantage of directly computing all the performance measures for each population in the network. MVA has the 
disadvantage of computing only average values, so it is impossible to construct the complete steady-state probability distribution directly from the results of MVA.

The convolution and MVA algorithms have comparable cost in terms of anount of computation and memory required [Agr85]. Although they are more efficient than the direct solution, their cost rises exponentially with the number of classes in the network. Recently, Conway and Georganas [CG86] developed a new algorithm called RECAL (Recursion by Chain Algorithm) for the computation of performance measures for closed multichain prod. uct form QNs, which involves a different recursion than the previous convolution and MVA algorithms. A new algorithm MVAC (Mean Value Analysis by Chain), using a similar recursion as RECAL but avoiding the computation of the normalizing constant, is presented in [CdL89].

Exact aggregation (decomposition) for closed product form QNs - Norton's theorem

A different approach to the exact solution of closed product form QNs is represented by the: aggregation (decomposition) method known as Norton's theorem. This is a non-iterative method, first proved in [CHW75] and extended in [SI82], that replaces a part of a network by a "flow equivalent server" with an appropriate service rate function (dependent on the number of customers), such that the equilibrium queue length and waiting time distributions in the rest of the system are the same as in the original system.

The efficiency of this method may be superior to the solution obtained by convolution or MVA if the size of the subnetwork $\sigma$ is chosen appropriately. A comparative cost analysis for networks with one class of customers found in [SI82] shows that the maximum gain by applying the Norton's theorem is obtained when the number of queues replaced by the "flow equivalent server" is about $40 \%$ of the total number of queues.

\subsubsection{Approximate solution methods for product form QNs}

As shown in the previous section, the cost of the exact algorithms for product form $Q N i$ can become prohibitive for sufficiently large networks, and the cost is associated with the recursions used in the solution. 
A possible approach to reduce the cost is to avoid the recursion in computation by using approximate instead of exact relations. A very simple approximation, due to Bard [Bar79], which breaks the recursive nature of the equation 2.4 is:

$$
L_{l j}\left(\mathbf{N}-\mathbf{e}_{k}\right)=L_{l j}(\mathbf{N})
$$

The accuracy of this approximation is not very good, especially for small populations [dLM84].

A better approximation proposed by Schweitzer in [Sch79], consiciers that the queue length for a population $\mathbf{N}$ reduced with one class $k$ job is proportional with the queue length for the complete population:

$$
L_{l j}\left(\mathbf{N}-\mathbf{e}_{k}\right)= \begin{cases}L_{l j}(\mathbf{N} ; & l \neq k \\ \frac{N_{k}-1}{N_{k}} L_{k j}(\mathbf{N}) & l=k\end{cases}
$$

The rationale for Schweitzer's approximation is that in case of large populations one would not expect to see significant changes in mean queue length by reducing a class population by one customer. This approximation has the disadvantage that, for small populations, the accuracy is not as good as for large populations.

A very accurate but more costly approximation, called Linearizer, was proposed by Chandy and Neuse in [CN82]. This is a refinment of Schweitzez's approach. The queue length for population $\mathbf{N}-\mathbf{e}_{k}$ is estimated as follows:

$$
L_{l j}\left(\mathbf{N}-\mathbf{e}_{k}\right)=\left(\mathbf{N}-\mathbf{e}_{k}\right)_{l}\left(F_{l j}(\mathbf{N})+D_{l j k}(\mathbf{N})\right)
$$

where:

$\left(\mathbf{N}-\mathbf{e}_{k}\right)_{l}$ is the population of class $l$ after the removal of one class $k$ customer from population $\mathbf{N}$;

$F_{i j}(\mathbf{N})=L_{l j}(\mathbf{N}) / N_{l}$ is the fraction of class $l$ customers at queue $j$ for population $\mathbf{N}$;

$D_{l j k}(\mathrm{~N})$ is the change in the fraction of class $l$ customers at queue $j$ resulting from the removal from population $\mathbf{N}$ of one class $k$ customer .

The proportionality assumption in Schweitzer's approximat:ion ' 3 equivalent to assuming that $D_{l j k}(\mathbf{N})=0$. The Linearizer algorithm estimates $\Omega$ t terating between populations $\mathbf{N}$ and 
$\mathbf{N}-\mathbf{e}_{k}$, for $k=1, \ldots, K$, and by assuming that $D_{l k j}\left(\mathbf{N}-\mathbf{e}_{k}\right)=D_{l k j}(\mathbf{N})$ (which implies that $F$ is a linear function of population).

The Schweitzer's and Linearizer approximations are by now standard approximations for product form QNs consisting of only SSFR and IS service centers.

A different approach to approximate solution of product form QNs is represented by a class of iterative methods applied to large multiclass networks, where the exact and approximate techniques previously discussed become prohibitive. Such an iterative method divides the network into a set of interrelated subnetworks, and then iterates betwren successive solutions for these subnetworks, until some convergence criterion is satisfied. In the iterative process, each subnetwork is analysed with the remainder of the network (the subnetwork's complement) represented in a simplified way. The complement's parameters for one submodel are obtained from the results of the other submodel. Every submodel has a product form solution, and is solved by one of the exact or approximate methods previously presented in this section. This type of iterative method has been used by de Souza e Silva, Lavenberg and Muntz in [dLM84, dLM86] to analyse a distributed system with many sit.es and many customer chains. A similar iterative techniques discussed in the next section, is also applicable to noa-product form QNs [dLM86, dM87]. Section 2.1.5 presents a general discussion about the principles and problems of using iterative methods to solve product form and non-product for QNs.

\subsubsection{Non-product form QNs}

Product form QNs naturally represent systems with independent customer behaviour and autonomous and fair servers [Agr85]. Independent customer behaviour means that the interaction among customers is limited to queueing for servers, and no other form of dependency between customers (as synchronization) is permitted. An autonomous serier uses only local information (such as local queue length) in its schaduling and service decisions, and dors not dipend on the state of other servers. A fair server does not discriminate against somcustomer classes depending on the queue length of other classes.

In software modelling we must be able to describe systems with nonautonomous and/or unfair servers or with dependency between customer behaviour, leading to queueing network models which do not have a product form solution. Unfortunately, the only exact inethord 
available to solve general networks with non-product form characteristics is the direct one: to determine and solve the corresponding Markov process. For the majority of practical problems, the direct method is either too costly, or intractable.

A lot of effort has been spent in developing approximate solution methods for a large variety of non-product form. QNs. These ad hoc methods are usually aimed to specific aspects and characteristics of different systems under consideration, making it difficult to give a general classification. The following list indicates some characteristics and behaviours found in computer systems that lead to non-product form QN models:

1. FIFO servers having service time with general distributions, or with exponential distributions but different means for multiple classes.

2. Priority scheduling. Lower priority class jobs have to suffer additional delay due to higher priority class customers arriving after them at the server.

3. Simultaneous resource possession. A job has to acquire and hold a certain resource (server) while using other resources . Examples are memory constrained systems (where memory should be acquired before the starting the execuition) and I/O systems (where both a channel as well as a device have to be simultaneously held).

4. Critical sections. A program has to acquire a semaphore or a lock before it can enter a critical section. While inside the critical section, it competes for the same resources as the other jobs.

5. Internal program concurrency. A customer (process) can spawn children processes (fork operation). The parent process may continue in parallel with its children or it may wait for them to finish before continuing its work (join operation).

6. Blocking. In system with limited queue size, a job will block a server if the next destination queue is full.

Since these problems are relevant to the goal of the present work, a brief review of some of the approximate methods for non-product form QN modelling systems with these characteristics is given in the following. Most methods are iterative techniques, thus section 2.1.5 gives a general discussion about the approximate iterative methods for QNs. 


\section{FIFO servers in non-product form QNs}

As shown in section 2.1.1, a FIFO server in a product form QN must rave an exponential service time distribution with the same mean for all customer classes. Normal extension for FIFO servers, as general service time distribution or different service time per class lead to non-product form QNs.

Marie developed in [Mar79] an iterative method for a single chain network of FIFO service centers with general service time distributions. The original network is decomposed in $h$ subnetworks, one for each such server. Each subnetwork is treated as a $M / G / 1$ queue with service time distribution having a rational Laplace transform. The effect of the rest of the network (subnetwork's complement) is represented as a state dependent arrival rate $t_{i}$ ii), that equals the throughput of the rest of the network with $(N-n)$ customers. In order to obtain $\lambda(n)$, Norton': theorem is applied to a product form QN that approximate the complement, by assuming that it has service centers with exponential service time and state: depender:r service rates. Marie's method has been extended to multiple chain networks by Neuve and Chandy [NC82], but it is clear that the computational cost becomes prohibitive, unless the number of chains, the population, and the number of non-product form servire: centers are very small.

Reiser presented in [Rei79] an MVA based approximation for FIFO service centers with chain dependent general service time distributions. The key assumption of this approxima-

tion is that the arrival theorem (as discussed in section 2.1.2) is still valid, i.e., an arriving customer observes the en "ibri $m$ solution for a reduced population having one less customer in the respective clas. Thus ine ipproximate algorithm is based on the MVA equations 2.2, 2.3, 2.4, where the lasi eruison that gives the waiting time is modified according to the followng heuris: ... The mea. raiting time $W_{k} j(\mathbf{N})$ of a class $k$ customer at FIFO server $j$ has three components:

1) The mean service time for the arrivir:g customer, $S_{k} j$.

2) The mean service time for the backlog of customers waiting (but not in service) at cinusu: $j$, approximated as:

$$
\sum_{i=1}^{K} S_{i j}\left[L_{i j}\left(\mathbf{N}-\mathbf{e}_{\mathbf{k}}\right)-\rho_{i j}\left(\mathbf{N}-\mathbf{e}_{\mathbf{k}}\right)\right]
$$


where $\rho_{i j}$ is the utilization of the server $j$ by class $i$ customers.

3) The residual service time of the customer in service at the arrival moment approximated as:

$$
\sum_{i=1}^{K} f_{i j}\left(\mathbf{N}-\mathbf{e}_{\mathbf{k}}\right) V_{i j}
$$

where $f_{i j}$ is the fraction of class $i$ throughput at queue $j$, and $V_{i j}$ is the mean residual service time of a class $i$ customer at center $j$ found from the renewal theory.

In order to break the recursive nature of MVA equations, [Rei79] also introduces an approximation for the change in mean queue size $L_{i j}$ when the population is reduced with one customer, that depends on the strength of the bottleneck for each class. (This is more complex than Schweitzer's approximation).

Another approach is proposed by Agrawal, Buzen and Schum in [ABS84], based on the response time preservation (RTP). RTP is introduced as a general technique for developing approximate analysis procedures, and is applied to some examples of non-product form ONs. Among them are QNs containing FIFO servers with general service time, or FIFO scrvers with different service times for multiple classes. In the first case, the general FIFO server in a single class QN is replaced by an equivalent server with exponentially distributed service time which insure the same response time for a job in the network as the original server. As an approximation to this condition, the response time for the equivalent server in isolation with Poisson arrivals is taken to be equal to the response time for the original server in isolation under the same arrival process. The accuracy is found to be reasonably for moderate coefficients of variations (ur to 5). In the case of multiple classes, the arrival process is different by class. The equival 'st server is considered to have PS discipline and different service time per class, determined from the condition that the response time for every class is preserved.

\section{Priority scheduling}

Computer systems containing devices at which some job classes have priority over others violate the fair service assumption necessary for product form solution. The priority policy may be Preemptive Resume (PR) or nonpreemptive Head Of Line (HOL). 
An early approximation that deals with PR servers is the reduced work rate approxinnation, used by Reiser in [Rei76]. This is based on the observation that at a PR server, lower priority customers "see" the server with a reduced average capacity for work because of the service on behalf of higher priority customers. Thus, the service rate for a class $j$ lower priority job is adjusted by multiplying the original rate by a factor $\left(1-\sum_{k=1}^{j-1} \rho_{k}\right)$, where $\rho_{k}$ is the utilization of the PR server by a class $k$ with higher priority than class $j$. The approach can be extended to networks consisting of several PR and IS centers, provided that the priorities of customer classes are at each PR are identical.

The shadow approximation introduced by Sevcik in [Sev77] can be seen as an algorithm to apply the reduced work rate approximation to a more general class of QNs. Each priority center is replaced by $J$ shadow centers, where $J$ is the number of priority classes. The service rate $\mu_{s j}$ of a class $j$ at its dedicated shadow center $s$ is adjusted to represent the average service rate for that class after the demand from the higher priority classes has been met:

$$
\mu_{s j}=\mu_{p j}\left(1-\sum_{k=1}^{j-1} \rho_{p k}\right)
$$

where $\mu_{p j}$ is the original rate for class $j$, and $\rho_{p k}$ is the utilization of the server by a higher priority class $k$. The equivalent $Q N$ with shadow servers substituted for every $P R$ server has a product form solution. An iterative process is necessary because, the exact values for utilizations $\rho_{p k}$ are not available when computing the adjusted rates. The accuracy of the shadow approximation has been assessed only for QNs with small number of centers and small populations; even so, the reported errors go up to more than 20 percent.

The method with the best accuracy for priority scheduling, dealing with both PR and HOL centers, has been proposed by Bryant et al. in [BKLC84]. This is an extension to the mean value analysis, that modify the formula for the waiting time at a priority server, in order to accommodate for the delay suffered by a lower priority class due to a higher priority class. Consider the arrival of a class $j$ customer (the tagged customer) to the priority center, That will have to wait for the completion of service for all higher and equal priority customers that are already in the queue, for the duration $\sum_{k=1}^{j} L_{k} s_{k}$, where $L_{k}$ is the class $k$ queise length. In addition, there is a delay due to the service of higher priority customers of class $k<j$ which arrive while the tagged customer is waiting and complete ahead of it; the mean value of this delay is $\sum_{k=1}^{j-1} W_{j} \lambda_{k} s_{k}=\sum_{k=1}^{j-1} W_{j} \rho_{k}$, where $W_{j}$ is the time spent by the tagged 
customer at the server, $\lambda_{k}$ is the class $k$ throughput, and $\rho_{k}$ is the server utilization by class $k$. Thus, the average waiting time spent by the tagged customer at a PR center is given by:

$$
W_{j}=s_{j}+\sum_{k=1}^{j} L_{k} s_{k}+\sum_{k=1}^{j-1} W_{j} \rho_{k}
$$

Equation 2.11 solved for $W_{j}$ provides the exact value for the waiting time spent by a class $j$ customer at a single $M / M / 1$ preemptive server with Poisson arrivals. However, in the case of PR server connected into a $Q N$, this only gives an approximate value for $W_{j}$, that is used for an approximate MVA analysis of such a net. [RKLC84] analyses the errors of the method: the largest errors (greater than 10\%) are found in wait time estimates for classes with lower priority, when the priority center utilization is high (more than 0.9 ).

In [EL88] is shown that the priority MVA method from [BKLC84] can be modified so that it utilizes approximate rather than exact MVA, without significant loss of accuracy.

\section{Simultaneous resource possession}

Simultaneous or overlapped resource possession in QN models for computer systems has most frequently arisen in two contexts:

a) limited multiprogramming level models in which a customer must gain admission to the multiprogramming set before competing for other resources as processors and $1 / 0$ devices;

b) I/O subsystem models in which a customer requires the services of a disk drive while seeking, searching and transferring data, and the additional services of a channel during the latter one or two operations.

Jacobson and Lazowska present in [JL82] an approximation method called surrogate delay applicable to single chain non-product form QNs consisting of several nonoverlapping instances oi simultaneous resource possession and any number of "normal" service centers (the rest of the system). An instance of simultaneous resource possession involves a customer who obtains a primary resource and holds it both for a preliminary service time $S_{P}$, and while queueing for and using some other resources from a secondary subsystem. (The total service time inside the secondary subsystem is $S_{S}$ ). The surrogate delay method iterates 
between two submodels. The first submodel, used to determine the delay due to primary resource congestion, contains a representation of the primary resource as a server with service time $\left(S_{P}+S_{S}\right)$, an approximation of the queueing delay caused by the secondary subsystem as a "surrogate delay", and a complete representation of the rest of the system. The second submodel, used to determine the queueing delay due to secondary subsystem, represents the secondary subsystem as a flow-equivalent server, and the primary resource as a "surrogate delay". The flow-equivalent server is obtained by analysing in isolation the secondary subsystem for a load $n=1, \ldots, N$, where $N$ is the population constraint imposed by the primary resource. The delay value for the surrogate in each submodel is estimated from the results obtained by solving the other submodel, and iteration is used.

Another iterative algorithm able to handle more general forms of simultaneous resource possession for QNs with passive resources is reported in [dM87]. A passive resource has a limited number of instances (or tokens), and is represented in the network by an allocate node (which has a queue for the jobs making a request), and a deallocate node. While: holding a passive resource, a job may visit any number of other passive or active resources. The method is applicable to multichain QNs, where jobs from different chains share the same passive resources. Furthermore, the use of passive resources can be nested. The technique contains FIFO/ an approximation for FIFO centers with multiple servers and different service requirements for jobs from different chains, needed to solve some submodels during the iteration. This method follows the general principles for approximate iterative methods shown in section 2.1.5, dividing the network in submodels and solving by iteration a set of equations having the form given in 2.13. The method clusters a network with passive resources into subnetworks as follows:

a) Form one subnetwork which contains all the resources (passive or active) visited by jolss while they are not holding any passive resource.

b) Form one subnetwork for each set of resources (servers) visited by jobs while they are holding a passive resource.

c) If a subnetwork $S$ found in step b) still contains a passive resource, go to step a), using $\mathrm{S}$ as a new network. 
The method represents the complement of a subnetwork in the following manner:

- The complement of a subnetwork found in step a) is represented for each passive resource by an IS center, which, combined with the allocate node, collapses to a FIFO multiple server center (where the number of servers is equal to the number of token for the corresponding passive resource). If multiple chains share the same passive resource and these chains have different load requirements in the complement, the mean service time will be different for each chain.

- The complement of a subnetwork found in step b) visited by $k$ chains that use dedicated passive resources is represented by $k$ closed chairs with limited population, and one IS server visited by all chains. However, if the $k$ chains use a shared passive resource, the complement is represented by a single closed chain (with limited population) with $k$ classes, and an IS service center.

- Due to the possibility of nested use of passive resources, a subnetwork on an intermediate level of nesting will have the complement represented differently for the higher and for the lower level.

The method has been applied to a variety of models; empirical results reported in [dM87] show that the algorithm has accuracy comparable with other approaches proposed in literature.

\section{Critical sections - serialization}

Critical sections and data base access are two examples of single-threaded or serialized processing. An QN model of a system with such behaviour does not have product form solution because the jobs simultaneously hold a "serialization token" and other servers. Although serialization is a particular case of simultaneous resource possession, the methods presented in the previous subsection are not directly applicable, because the resources are not used in a nested fashion: the jobs holding the serialization token may compete for the same resources as the other jobs.

Agrawal and Buzen present in [AB83] the aggregate server method to analyse serialization delays for closed QNs with a single job class and load-independent servers. The method 
builds an approximate product form model that uses an "aggregate" server to represent both the service time and queueing delay spent by jobs in each serialization phase (critical section). When a job is in a critical section, it delays other jobs because it still contends for other servers. Thus, the transformed model also replaces the original servers by slower shadow servers. Both the service time for the aggregate servers and the adjustment factors for the other servers are iteratively determined by successive solution of the approxinlate model. The aggregate server method is extended for multi-class mixed networks, in which the critical sections are not shared between jobs in different classes.

Another approach presented by Jacobson and Lazowska in [JL83] is intended to solve multiple class QNs with serialization delays by using transformations that represent population constraints. The method iteratively uses a two level model for the system. The low level submodel includes only the serializable servers (that may be visited by jobs in serialized phase), and it is used to compute critical section residency time given a fixed population equal to the average number of serialized and non-serialized customers using these servers. The high level submodel includes the non-serializable servers plus some new servers representing the time spent in critical sections and the time spent at serializable servers by non-serialized jobs. This submodel is used to determine the average aumber of customers inside each critical section and outside all critical sections. The algorithm uses non-integer values for mean population sizes, allowed by the Schweitzer's approximation to the MVA technique that is used to solve the product-form submodels during the iteration.

\section{Internal concurrency}

As seen in the previous sections, the QN models for computer systems usually represent the software load as customers and the hardware resources as service centers. If we want to capture in QN models the characteristics of the software, we often have to represent some kind of dependency between customers, such as the splitting of a job(process) in tasks(children processes), that will be executed concurrently, and may wait for each other at the end. 'This kind of dependent behaviour also leads to non-product form QNs.

Heidelberger and Trivedi have developed in [HT82] an approximate model for analysing asynchronous concurrency (i.e. fork but no join). The processing system is composed of a fixed set of servers. A job consists of one primary task and zero or more statistically identical 
secondary tasks, spawned by the primary task at some points of its execution, when it visits a special server. Except for queueing effects, the primary and secondary tasks execute independently of each other and do not require synchronization. The model represent the behaviour of primary tasks as an closed chain and of secondary tasks as an open chain. The approximation proposed in [HT82] assumes that the arrival process for the open chain is a Poisson process independent of the state of the network. A nonlinear equation relates the closed chain throughput and the open chain arrival rate, whose solution is found by iterating through a sequence of product form QNs. The conditions for a stable solution and throughput bounds are also determined. The model is used for two type of systems: a central server system with overlapped $\mathrm{I} / \mathrm{O}$ and a terminal oriented timesharing system where batch tasks are forked from time to time.

A more complex type of task dependency is modelled by th: same authors in [HT83]. The program behaviour is such that a job (or primary task) can fork into two or more concurrent secondary tasks at some point during its execution. Except for queueing effects, the secondary tasks execute independently, and at the end of their exerution they wait for for all of their siblings to finish before merging with their parent (join operation). The model consists of a finite number of servers and a pseudoserver(node 0 ) where the split and join operations take place. (After a split, the primary task remains inactive at pseudoserver until all its children finish and join). Two approxin nation techniques to solve such models are proposed in [HT83].

The first is a decomposition approximation. Assuming that the primary and secondary tasks visit many servers before being implicated in a split or join operation that change the number of tasks in the system, it is reasonable to assume that the steady-state is reached for a given population before a population change will occur. This gives the rationale for a hierarchical decomposition into a two-level model. The lower level model is a productform QN with fixed population, that will be solved for all feasible combinations of primary and secondary task populations. The higher level model is a finite state Markov chain having a state for every feasible combination of primary and secondary task population. B: aggregating togetiner all the states of the complete Markov chain for the whole system that have the same population vector, it results a set that represent one state of the higher level model. The transition rate for this Markov chain is approximately determined by assuming 
that the secondary tasks complete in random order and that the set of waiting tasks is uniformly distributed over the state space. The stationary distribution for the Markov chain is obtained by directly solving the balance equations. The mean performance values for the whole model are obtained from the performance values for the lower level models, by weighting them with the aggregated state probabilities.

The second approximation is similar to the method of surrogate delays proposed in [JLi2]. The primary tasks may be in one of two macrostate: active or waiting; the secondary tasks may be active, waiting or dorment. The approximation uses fictitious delay servers to represent the waiting and dormant states for every type of tasks. The transformed model has a product form solution. This approximation neglects the correlation between different task macrostates (for example, a primary task is waiting while its secondary tasks are artive and/or waiting, etc). The approximate solution is obtained by iteratively solving a sequence of product form QNs. Comparing the two approaches used in [HT83], the decomposition approximation was found to be more accurate than the complementary delay approximation. However, the first method has the disadvantage that the space state of the Markov chain (high level model) grows rapidly with the population, and the approximate procedure to build it is difficult to be generalized.

Another example of an hierarchical decomposition approach applied to the analysis of a QN model for a system running non- independent tasks is presented by Thomasian and Bay in [TB85]. The workload is a batch system composed of a set of tasks with precedence. relationships, defined by an acyclic graph. A task visits many devices before it completes, thus it is assumed that a steady state is reached before any task ends. Applying the deconnposition principle, a "high level" model is constructed as an acyclic Markov chain which has as states all possible combinations of simultaneously active tasks. For each of these stattes, in "low level" product form QN model is analysed in steady-state, using its results to detrrminc" the transition rates for the high level Markov chain model. The overall completion time for the tasking system is found from the high level model as a last step. This solution appristch has been used to study task allocation problems in distributed system. The accuracy of the algorithm is claimed to be good. 


\section{QNs with blocking}

In recent years, queueing networks with blocking have become an important research topic in performance evaluation. This is mainly due to the fact that in actual computer systems the resources (such as message buffers) have finite capacity, so they are appropriately modelled as service centers with a finite length queue. An important feature of systems with finite buffers is that a server may become blocked when the capacity limitation of the destination queue is reached. Several types of blocking have been studied. According to [Aky89] there are three classes of blocking.

Type 1 - Transfer Blocking. Upon completion of its service, a job at station $i$ attempts to enter destination station $j$. If station $j$ is full at that moment, the job is forced to wait at server $i$, blocking it until station $j$ can be entered. This type of blocking has been used to model flexible manufacturing systems and disc $1 / 0$ subsystems.

Type 2 - Service Blucking: A job in station $i$ declares its destination $j$ before its service starts at $i$. If station $j$ is full, server $i$ is blocked until a departure from $j$ occurs. At this moment, $i$ becomes unblocked and starts its service. This blocking type has been used to model production lines and telecommunication networks.

Type 3 - Rejection Blocking: Upon service completion at station $i$, a job attempts to join destination station $j$. If station $j$ is full at that moment, the job is refused. The rejected job goes with a certin probability back to ser"er $i$ and immediately receives a new service. This is repeated until station $j$ becomes available. This blocking type has been used to model communication networks.

Comparisons between these types of blocking is given in [OP89]. A bibliography concerning QNs with blocking is given in [Per84]. Under some very restrictive conditions, a closed QN with type 1 of blocking has product form solution. These conditions are:

- the network has exactly two nodes (shown by Akyildiz in [Aky87])

- the number of customers in the network is equal to the capacity of the smallest buffer plus one (shown by Onvural and Perros in [OP89]). 
Among the approximation algorithms for different types of QNs with blocking are the following: Suri and Diehl [SD84], Onvural and Perros [OP88]- approximation for cyclic QNs with type 1 and 2 of blocking; Akyildyz [Aky88, Aky89] - mean value analysis approximation for QNs with type 1 of blocking ; Perros, Nilsson, Liu [PNL88] - approximation for closed QNs with infinite and finite queues using a variant of Norton's theorem.

\section{Software servers modelled by QN approach}

There are two different approaches in literature that we are aware of (beside the work on SRVN) that model software servers.

In the first article, [Rol88], Rolia models a hierarchy of processes in which a process may only request service from processes on the level immediate below it. A client process remains blocked until the service is completed. The processes at the top level play only the role of clients, while all the other play a dual role of "client/server" as described in section 1.1.2. However, there is no "post-service" phase, (i.e. no service given by the server for witich the client does not have to wait), and the servers do not offer a range of services with different workload requirements, such that each client can request several of this services. The model takes into consideration the competition for processors, whose scheduling may be with or without priorities. The solution method is an iterative one: the model is decomposed into interrelated submodels, such that each submodel is a QN and can be solved by by MVA. The delays due to nested service are transferred as surrogate values between submodels. Rolia's work represents the closest approach to SRVN models, the basic differences being the absence of a "post RV" phase and of server entries.

In the second article, [Fon89], Fontenot extends the QN notion of a service station by introducing the "mobile server" that represents software servers playing the dual role of "client/server", as described in section 1.1.2. The work in [Fon89] was motivated by practical problems of "software congestion" (described in section 1.1.2 as "software bottleneck") observed in a messaging-server prototype. However, the solution approach proposed in [Fon89] for an open system with a single mobile server is based on heuristic observations, and thus very difficult to generalize for more complex systems. 


\subsubsection{General discussion about approximate iterative methods for QNs}

As seen in the previous sections, a variety of iterative methods have been described in literature for both product form and non-product form QNs. This section presents a general discussion, based on [dLM84, dM87, Agr85], about the principles of using iterative techniques to find approximate solutions for QNs, and about the problems implied by these methods.

The approach common in all iterative approximation techniques for QNs is to decompose the original system into a set of interrelated subnetworks. For each subnetwork a submodel is built, containing that subnetwork and a simplified representation of the rest of the system (the subnetwork's complement). The iterative nature of the solution comes from the fact that the parameters of the simplified complement of a subnetwork are modified as a function of the results obtained for other submodels . More formally, we solve by iteration a set of (generally nonlinear) equations:

$$
\begin{gathered}
\text { Sol }_{i}=f\left(\text { Par }_{i}, \mathrm{CPar}_{i}\right) \quad i=1, \ldots, M \\
\text { CPar }_{i}=g\left(\text { Sol }_{j}, \ldots, \text { Sol }_{k}\right) \quad i=1 \ldots, M ; j, \ldots, k \neq i
\end{gathered}
$$

where:

$M$ is the number of submodels.

$S o l_{i}$ is the solution of submodel $i$

Par $_{i}$ is the set of parameters for the subnetwork ${ }_{i}$ taken from the original set of parameters

$\mathrm{CPar}_{i}$ is the set of parameters for the simplified complement of subnetwork

Within this general framework, the modeller has a rich set of choices (usually made heuristically):

a) The choice of subnetworks: they may be disjoint or may overlap. Also, a subnetwork may be a single server or a cluster of servers.

b) The choice of simplified representation of the complement of a subnetwork for eact. chain. Some possibilities are: 
- queue size dependent arrival rates [Mar79]

- infinite server [JL82, Zah83, dLM84, dLM86, dM87]

- reduced customer population [JL83]

- state dependent Poisson arrivals [dM87]

- center with queue dependent service rate [JL82]

- FIFO single server [Zah83]

- FIFO multiple strver [dM87]

For multiple chain networks, the complement may be represented differently for different chains [dLM84, dI.M86].

c) The choice of method to determine the parameters for the complement for a given representation. For example, for a Poisson arrival representation the arrival rate may be chosen in two ways:

- equal to the throughput of that chain computed in a previous iteration

- equal to the value that yields the same population in the subnetwork for that chain (what needs some additional computation).

d) The choice of how the final estimates of the results for the whole network are obtained. This choice arises because the same performance result may be represented in morr than one subnetwork, and we may get more than one value for it.

The criteria for making these choices are:

- the accuracy of the results

- the computational cost

- the generality of the method.

Usually, the accuracy must be evaluated empirically, since so far error bounds have wot been obtained for the vast majority of approximate iterative networks. For cost reasons, 
the submodels should have product form . Also, the parameters of a complement should be easily obtainable from the results of the other submodels.

Another problem is the difficulty to establish the existence and uniqueness of solutions for simultaneous nonlinear equations, and to prove the convergence of a particular iterative solution method. A useful theorem for establishing existence is the Brouwer fixed point theorem, whicin has beer: used in [HT83, dLM84, ES84, Agr85]. Uniqueness and convergence can be proved by using the Contraction Mapping theorem and the Newton theorem (see [dLM84, Agr85] for a discussion). A study of the accuracy, speed, and convergence behaviour for approximate MVA algorithms for product form QN is conducted in [ZES88], that also suggests a new stopping criterion and a simple extrapolation techniques to speed up the convergence. The conclusion is that, in general, these properties are very difficult to prove, and therefore, most iterative methods used to solve QN models are empirically assessed by applying them to a large number of cases.

\subsection{Solution methods at the Markov chain level}

Previous methods for software modelling in [Rol88], [WNPM89] were based on some of the QN approximations described in the previous section. In search of better accuracy, the present research is based on solution at the Markov Chain level.

\subsubsection{Markov chain aggregation-disaggregation}

The QN solution methods presented in the previous sections avoid the construction of the complete underlying stochastic process for the QN models that are analysed. Another approach to find the solution of QN models is to build the corresponding Markov chain model, and then to reduce by some methods the computational burden of solving the linear set of global balance equations. A possibility to achieve this has been to "decompose" the Markov chain by aggregating groups of states into smaller chains, solving the smaller chains, and then recombining these solutions to obtain the overall solution [Sch84], [FC87].

The main advantage of this method, named aggregation-disaggregation, is the reduction of the computational intensity and of the memory requirements, since the solution for one large system is found by solving a certain number of small systems. 
Another advantage of the method is a theoretic one: the aggregation can provide a rigorous justification of decomposition as a tool for analysing systems, giving insight into the choice of the aggregate parameters, as opposed to other heuristic simplifications. It also gives a more rigorous base for abstraction, making it possible to analyse a system from different perspectives and to integrate the different views into a global one.

The aggregation-disaggregation algorithm introduced by Takahashi in [Tak75] and discussed in [Sch84], is briefly presented in this section. Although the algorithm is applicable. for both discrete and continuous time $\mathrm{MC}$, we will consider here only the latter case.

The Kolmogoroff equations for a Markov chain in continuous time are:

$$
p_{i} \sum_{j \in \Omega i, j ! !} \lambda_{i j}=\sum_{j \in \Omega, j i} p_{j} \lambda_{j i}, \quad \sum_{i=1}^{N} p_{i}=1
$$

where $\Omega=\{1, \ldots, N\}$ is the finite state space with $N>>1, \mathbf{p}=\left\{p_{1}, \ldots, p_{N}\right\}$ is the steady state probability vector, and $\lambda_{i j}$ are the transition rates.

By aggregation, the $N$ states of the initial $M C$ are grouped into $M$ blocks $\Omega_{\alpha}, \alpha=$ $1, \ldots, M$ where $M \ll N$, such 'h hat $\cup_{\alpha=1}^{M} \Omega_{\alpha}=\Omega$ and $\bigcap_{\alpha=1}^{M} \Omega_{\alpha}=0$ (see Figure 2.1). The algorithm seeks to compute the aggregate probability $P_{\alpha}$ for each block $\Omega_{\alpha}$ such that:

$$
P_{s}=\sum_{i \in \Omega_{a}} p_{i}
$$

The aggregate transition rate $\xi(\mathbf{p})_{\beta \alpha}$ between two aggregated states $\Omega_{\beta}$ and $\Omega_{\alpha}$ is a weighted sum of the original rates $\lambda_{j i}$ for $j \in \Omega_{\beta}$ and $i \in \Omega_{\alpha}$, conditioned on being in block $\Omega_{\beta}$ :

$$
\xi(\mathbf{p})_{\beta \alpha}=\sum_{j \in \Omega_{\beta}} \sum_{i \in \Omega_{\alpha}} p_{j} \lambda_{j i} / P_{\beta}
$$

There is also defined an aggregated rate $\xi(p)_{\beta i}^{*}$ between the aggregated state $\Omega_{\beta}$ and the original state $i$ :

$$
\xi(\mathbf{p})_{\beta i}^{*}=\sum_{j \in \Omega_{\beta}} p_{j} \lambda_{j i} / P_{\beta}
$$

Takahashi's algorithm replaces the balance equations 2.14 of the original MC with the following two sets of equations:

$$
P_{\alpha} \sum_{\beta \neq \alpha} \xi(\mathrm{p})_{\alpha \beta}=\sum_{\beta \neq \alpha} P_{\beta} \xi(\mathrm{p})_{\beta \alpha} \quad \sum_{\alpha=1}^{M} P_{\alpha}=1
$$




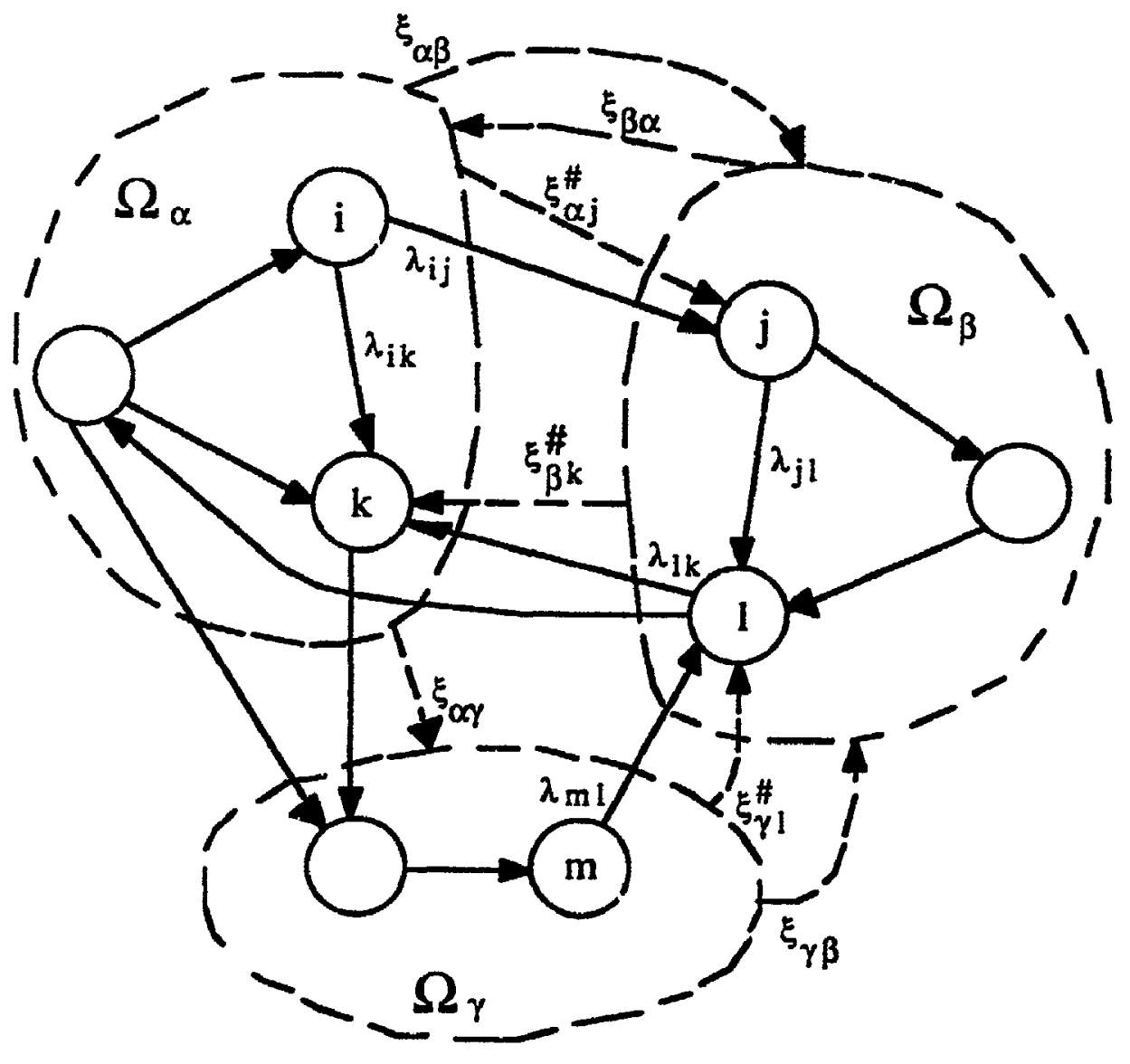

Figure 2.1: Markov Chain aggregation 


$$
p_{i} \sum_{j \in \Omega, j i} \lambda_{i j}=\sum_{j \in \Omega_{\alpha} / i} p_{j} \lambda_{j i}+\sum_{\beta \neq \alpha} P_{\beta} \xi(\mathrm{p})_{\beta i}^{\#}, \quad i \in \Omega_{\alpha}, \quad 1 \leq \alpha \leq M
$$

The first set of equations 2.18 represent the aggregation aspect, showing that $\mathbf{P}$ is the equilibrium probability distribution for the aggregated MC. The second set 2.19 represents the disaggregation aspect, giving the equilibrium probabilities $p_{i}$ for the states $i \in \Omega_{a}$ in terms of aggregated rates and probabilities for states $\Omega_{\beta}, \beta \neq \alpha$, and nonaggregated rates and probabilities for states $j \in \boldsymbol{\Omega}_{\alpha}$. An iterative method alternating betwcen these two equations produces the solution $\mathbf{P}$ for the original $\mathrm{MC}$, and $\mathbf{P}$ for the aggregated $M C$.

\subsubsection{Nearly decomposable systems}

Another approach to solve a special class of complex Markovian models is based on the concept of near complete decomposability proposed by Simon and Ando in 1961 for general systems [SA61], and applied later to queueing systems by Courtois [Cou7i].

The concept of nearly decomposable systems is based on two fundamental observations [Cou85]:

- Frequently, complexity takes the form of hierarchy, whereby a complex system is composed of interrelated subsystems that have in turn their own subsystems, etc.

- In general, interactions inside subsystems are stronger and/or more frequent than interactions among subsystems.

Because of this difference between strong and weak coupling, a nearly decomposalile structure behaves differently in short-term and long-term periods, as shown by Simon and Ando in two theorems that can be summed up as follows:

$\therefore$ In short-term period, as a result of stronger internal bonds, subsystems tend to reach an internal equilibrium "approximately" independently of one another.

2. In a long-term period, when a whole system evolves toward a global equilibrium st.at.. under the influence of weak interactions among subsystems, the internal equilibriun reached at the end of the short-term period are approximately maintained in relativ. value. 
The Simon-Ando theorems provide a rigorous foundation for decomposing a system, considering the components in isolation, and then studying the interaction between systems in a simplified way.

More formally, the finite non-negative stochastic transition matrix of a nearly decomposable system, after appropriate row and column permutations, can be put under the following form that shows the decomposition into $N$ subsystems:

$$
\mathbf{Q}=\left|\begin{array}{cccc}
\mathbf{Q}_{11} & \mathbf{Q}_{12} & \cdots & \mathbf{Q}_{1 N} \\
\mathbf{Q}_{21} & \mathbf{Q}_{22} & \cdots & \mathbf{Q}_{2 N} \\
\vdots & \vdots & \ddots & \vdots \\
\mathbf{Q}_{N 1} & \mathbf{Q}_{N 2} & \cdots & \mathbf{Q}_{N N}
\end{array}\right|
$$

where

$Q_{1 I}$ is a square diagonal submatrix representing the internal transitions inside the subsystem $I$, where $I=1, \ldots, N$;

Q1J, where $I \neq J$ is an off-diagonal submatrix whose elements are small compared to those of the diagonal submatrices $Q_{\mathbf{n}}$.

The stochastic matrix $\mathbf{Q}$ can be written as:

$$
\mathbf{Q}=\mathbf{Q}^{*}+\epsilon \mathbf{C}
$$

where:

$\mathbf{Q}^{*}=\operatorname{diag}\left(\mathbf{Q}_{\mathbf{1}}^{*}, \mathbf{Q}_{2}^{*}, \ldots, \mathbf{Q}_{\mathbf{N}}^{*}\right)$ is a diagonal stochastic matrix partitioned in the same way as $Q$ with zero off-diagonal submatrices;

$\mathbf{C}$ is a square matrix of the same order as $\mathbf{Q}$ which has the property of keeping both $\mathbf{Q}$ and Q* stochastic;

$\epsilon$ is a real positive number, small compared with the elements of $Q^{*}$, called degree of coupling.

The aggregation procedure finds approximate values for the vector of steady-state probabilities of the Markov chain defined by $\mathbf{Q}$ as follows: 
a) Find the short-term equilibrium probability vector $\gamma_{i}^{*}$ for every subsystem described by a diagonal submatrix $Q_{I}^{*}, I=1, N$ analysed in isolation.

b) Determine an aggregate matrix of transition probabilities between subsystems, by using the off-diagonal submatrices $Q_{I J}, I \neq J$ and the results from the first step.

c) Find the steady-state vector $\mathbf{X}=\left(X_{1}, X_{2}, \ldots, X_{N}\right)$ for the aggregate matrix built in sten b). The vector $\pi=\left(X_{1} \gamma_{1}^{*}, X_{2} \gamma_{2}^{*}, \ldots, X_{N} \gamma_{N}^{*}\right)$ represents the approximate steady-stat. probability vector for the whole system.

The accuracy of the aggregation method has been studied and error bounds have le'" established. Courtois has shown that in general, the error remains small, of the same orcler. of magnitude as the degree of coupling $\varepsilon$. Vantilborgh has found an improved approximation, reducing the error at $O\left(\epsilon^{2}\right)$ [Van85]. For product form queueing networks the method yields exact results. Vantilborgh has shown in [Van78] the conditions for exact aggregation in exponential QNs.

\subsection{Petri Net models and solution methods}

Basic Petri net (PN) models have been developed with the specific purpose of representing in a natural way synchronization and cooperation among concurrent processes. The formal definition of a PN is the following [AMBC84]:

$$
\begin{aligned}
P N & =(P, T, A, M) \\
P & =p_{1}, p_{2}, \ldots, p_{n} \\
T & =t_{1}, t_{2}, \ldots, t_{n} \\
A & \subseteq P \times T \cup T \times P \\
M^{\prime} & =m_{1}, m_{2}^{\prime}, \ldots, m_{n}^{\prime}
\end{aligned}
$$

where $P$ is a set of places, $T$ is a set of transitions, $A$ is a set of directed arcs and $1{ }^{\prime \prime}$ is the initial marking (initial state). Arcs connect places to transitions and transitions $1 .$. iacess. Places may contain tokens which are moving through the net. The state of a PN $(a, a l i c .1$ its marking) is defined by the number of tokens contained in each place. A transition is t mabled 
when all of its input places contain at least one token. Enabled transitions can fire, thus removing one token from each input place, and placing one token in each output place. Each firing of a transition modifies the distribution of tokens on places, and thus produces a new marking '. $\mathrm{PN}$. The reachability set in a PN with a given initial marking $M^{\prime}$ is defined as the set of all markings that can be reached from $M^{\prime}$.

The basic PN models can be analysed for logical (qualitative) properties such as liveness (absence of deadlock and livelock), $k$-boundedness (the maximum number of token in any place is less than or equal to $k$ ), safeness (maximum one token in any place), etc. No quantitative perfornance measures can be obtained from these models since time is not considered in basic PNs.

\subsubsection{Stochastic and Timed Petri Nets}

Several authors have proposed augmented PN models which include temporal specifications, so that performance evaluation becomes possible. Time has been added to PN models in two ways:

1. a) Stochastic PN (SPN) uses exponentially distributed time intervals

2. b) Timed PN (TPN) uses deterministic delays having a fixed number of discrete time intervals.

The following subsections present briefly different developments in the domain of stochastic and timed PNs.

We should also mention here the work on Stochastic Activity Networks (SAN) led by Meyer [MMS85], [SM87]. SAN are similar to SPN, in the sense that all PN basic constructs have equivalents in SAN. The difference is that SAN allow also the definition of predicates and functions (i.e. enabling predicate, input function, reactivation function) that have an important role in the behaviour of the net, which makes the model description more compact and cryptic.

\section{Stochastic Petri Nets}

The Stochastic Petri Nets (SPN) have been independently introduced by Natkin [Nat80] and Molloy [Mol82], and extended by Ajmone Marsan et al. in [AMBC84] (GSPN), and 
in [AMC86] (DSPN). All these PN classes (SPN, GSPN, DSPN) belong to a category of PNs that associates time with transitions. The transition firing operation is atomic, i.e., the removal of tokens from input places and the addition of tokens to output places is a single indivisible operation. The delay associated with a transition represents the time int erval for which the transition must remain enabled before it can fire. The property of atomic firing brings some advantages to SPNs compared with other PN classes where the firing is not atomic [AMBCC87]:

- the state definition is identical to that of untimed PNs, which facilitates the use of the results of classical PNs theory in the field of stochastic PNs;

- a broader class of conflicts may be represented, and the resolution of conflicts can be. based on temporal specifications.

The Stochastic Petri Nets (SPN) described in [Mol82] are obtained by associating with each transition an exponentially distributed delay named firing time. The formal definition of SPN is the following:

$$
S P N=\left(P, T, A, M^{\prime}, R\right)
$$

where $P, T, A$, and $M^{\prime}$ are the same as in 2.22 , and

$$
R=\left(r_{1}, r_{2}, \ldots, r_{m}\right)
$$

is the set of firing rates (possible marking dependent) associated with the iransitions.

Molloy has shown that SPNs are isomorphic to continuous time Markov Chains, duce to the memoryless property of the exponential distributions of firing times. SPN markings correspond to MC states. The semantics of SPN can be described by means of a race model. When a marking simultaneously enables several (conflicting or concurrent) transitions, the. next marking change will be due to the transition whose firing delay is minimum, i.e., that wins the race with other transitions. The sojourn time in a marking (state) 11 is an exponential distributed random variable with average $\left(\sum_{i \in H} r_{i}\right)^{-1}$, where $U$ is the set of transitions enabled by marking $M$. If $H$ contains more than one element, the probability that a certain transition $t_{k} \in H$ will actually fire (win the race) can be obtained as:

$$
P\left(t_{k} \mid M\right)=r_{k} / \sum_{i \in H} r_{i}
$$


The evaluation of the steady-state probability distribution is performed by using classical techniques for the analysis of Markovian processes.

Generalized Stochastic Petri Nets (GSPN) [AMBC84] try to cope with the problem of state space explosion by defining two types of transitions: timed and immediate. An exponentially distributed firing time is associated with timed transitions, while the immediate transitions fire in zero time with priority over timed transitions. GSPN is still equivalent to a Markov chain model, and their solution is less expensive than an topologically identical SPN . The stochastic process generated by the execution of a GSPN spends a non-null time in markings enabling timed transition only (tangible markings), while it transits in zero time through markings enabling at least one immediate transition (vanishing markings). The latter class of markings can be removed from the state space, generating a reduced Markov chain, whose transition rate matrix can be easily oblained, and whose solution is less expensive.

Deterministic and stochastic PN (DSPN) proposed in [AMC86] by Ajmone Marsan and Chiola is a further extension to GSPN. DSPN integrate in the same model timed transitions with deterministic and exponentially distributed firing delays. Under the restriction that no more than one deterministic transition is enabled in any marking, it is possible to map the DSPN on a semi-Markov process, which will be analysed using an approach quite similar to the embedded Markov chain technique. A discrete-time Markov chain is defined by sampling the co:atinuous time process at the instants of deterministic transition firing (if such a transition is enabled in a marking), and at the instants of firing of exponential transitions. The embedded Markov chain states do not match exactly the markings of the nets. After finding the steady-state probability distribution for the Markov chain, this will be mapped into a probability distribution for the marking set.

\section{Timed Petri Nets}

Timed PNs associate with the elements of the net deterministic delays that have a given number of discrete time intervals. This types of PNs have been introduced with the declared purpose to model computer hardware.

Different versions of Timed PNs have been introduced in literature: some of them associate a deterministic delay with places (as proposed by Sifakis in [Sif79], others associate a 
delay with transitions (as proposed by Zuberek in [Zub80], by Razouk and 'ielps in [RP8.4], by Molloy in [Mol85], or by Holliday and Vernon in [HV87]).

The timing distributions and/or the structure of the net state space are generally restricted so that a discrete-time Markov chain model can be built (that unfortunately has a bigger space state then the marking space of the net). Standard analysis techniques for Markovian models can be applied to analyse the net performance.

\subsubsection{Approximate solution methods for Stochastic Petri Nets}

As shown in the previous section, SPN and their generalized versions are equivalent with underlying stochastic processes described by Markov or semi-Markov chains, which are pro. duced automatically from the net model definition. Thus, the standard analysis technicuues for this class of models can be applied to solve the Markovian model and compute performance measures of interest.

Unfortunately, the direct solution (that implies building the Markov chain and solving the Kolmogoroft equations) is difficult for two reasons:

a) the state space grows exponentially with the complexity of the model, becoming very large (or worse, intractable) even for models of moderate size;

b) when the Markov chain can be obtained, solving it is a computationally intense process that makes the direct solution expensive.

The field of PN with temporal properties is much younger than the field of queueing, networks, which partially explains the lack of efficient techniques for temporal PN, while: QNs enjoy such a rich assortment of fast solution techniques [VZL87]. Another reason" is that, due to the low level of abstraction of the PN basic building blocks, the underlying MC model is less regular than in the $Q N$ case, and thus it is more difficult to find and take: advantage of such regularities in the solution techniques. In the following subsections in few approximate Inethods for SPN found in literature are briefly presented.

\section{State-space decomposition}

This approach can be applied to speed up the process of solving the Markov chain, once the state transition rate matrix has been -onstructed. 
Giglmayr presents in [Gig87] a hierarchical decomposition method based on the concept of nearly decomposable systems [Cou77], that is used to find the stationary marking probabilities for stochastic PN. The paper presents a series of decomposition rules based on the sparseness of the transition rate matrix, that save computation effort. The method is claimed to give exact results for any type of SPN. The effort to find appropriate decompositions does not seem to be taken into consideration when analysing the efficiency of the algorithm.

\section{Model level decomposition}

Because the cost of model analysis grows exponentially with the size of the model, an exponential improvement can be obtained by partitioning the model into submodels, if each can be analysed independently of the others.

Ammar and Islarn propose in [AI89] a time scale decomposition technique for a class of GSPN models comprising activities whose duration differs by orders of mag cude (that generate "stiff" Markov chains). The decomposition is based on the theory of nearly decomposable systems [Cou77], and is equivalent to applying the state aggregation process to the underlying Markov chain that represents the behaviour of the GSPN. The advantage of the proposed algorithm is that the decomposition is automatically operated at model level, and not at transition matrix level. Thus, the construction of the whole state space is avoided . The given system is decomposed into a hierarchical sequence of aggregated subnets each of which is valid at a certain time scale. The aggregation at each level is done by assuming that the transitions included in the lower ler al are immediate. The proposed algorithm has a good accuracy, depending on the degree of coupling, that can be determined at model level. The time scale decomposition method has been applied for performance analysis of systems with random failures and repairs.

Another approach for decomposition of GSPN at model level, taken by Ciardo in [Cia89], is based on the concept of near-independence of the networks, which is orthogonal to the concept of near-decomposability [Cou77]. Although Ciardo does not attempt a formal characterization of the level of independence (which seems to be more difficult than the characterization of the level of decomposability), he considers different types of dependence between GSPN subnetworks, studies their "near-independence" property at Markov chain level, and then gives rules to exploit the near-independence at GSPN model level. The examples 
of "real-life" interactions among GSPN subnets for which near-independence decomposition algorithms are proposed are the following: one-way condition testing, processor-sharing, rendezvous, parbegin-parend, and producer-consumer. The accuracy is empirically determined by applying the decomposition method to analyse different models (i.e., the availability of a distributed file system, the throughput of an Ada tasking system, and the productivity of a flexible manufacturing system).

\subsection{Literature Review Conclusion}

The goal of this chapter was to review the work in the field of analytical performance modils relevant to software modelling in general, and to SRVN models in particular. The two main modelling techniques, QNs and PNs, have been studied from two viewpoints: the ability to capture important features of the concurrent software, and the efficiency of their solution methods. Also, a section was devoted to solution techniques at the Markov chain level, that. can be applied to analytical models that are iso torphic to a Markov chain.

QNs have been extensively used for analytical computer performance modelling, their incontestable advantage being the combination of expressive power (due to the high level of abstraction of the basic building blocks) and solution efficiency (that avoids the exponential explosion of the state space). However, QNs fail to capture in a direct way important f(atures of the concurrent software, that lead iu non-product form models. A lot of effort has been spent in developing approximate solution methods for a large variety of non-product form QNs. These ad hoc methods are usualiy aimed to specific aspects and characteristics and are difficult to generalize. It is very difficult to capture in a $Q N$ model the following characteristics of a software server, presented in section 1.1.2: client/server duality of software objects due to multiple layers of service, internal concurrency due to "post-service" phases, multi-class service combined with FIFO scheduling.

- The client/server duality of software servers was modeled in [Rol88] and [Fon89]. [36t, h use surrogate delays to include in the service time of a server the delays due to nestred services. The same approaches is taken in all SRVN algorithms.

- The "post-service" phase offered by a software server, increasing the internal cuncur. rency, could be assimilated to the model of asynchronous concurrency (i.e. fork but. 
no join) modelled in [HT82], where secondary jobs are "spawned" by a primary job. This model can represent the extra processing load due to the parallel execution of the software server and client (that are both jobs in the QN model), but does not capture two important elements: the extra queueing suffered by the client for the software server, and the synchronization effect between a client and the software server, that makes possible that a new request from a client has to wait until the servers finishes the "post-service" phase for the previous request from the same client.

- The combination of multi-class service and FIFO service leads to non-product QN, as shown in subsection 2.1.4. There are several approximations dealing with this problem, as reviewed in 2.1.4. The heuristic SRVN method uses the approximation proposed in [Rei79].

The QN approximations for QN with blocking, reviewed in subsection 2.1.4, and these for priorities, reviewed in subsection 2.1.4, could be also used for software modelling. For example, the SRVN algorithm presented in [MWNP89] and [WNPM89] use the priority approximation from [BKLC84].

The SRVN model integrates all these features together. The heuristic MVA method for SRVN ([Woo89], [WNPM89]) uses approxirnations based on QN theory (MVA framework, surrogate delays as in [JL82] for nested service, FIFO approximation techniques from [Rei79], priority approximation from [BKLC84]) plus some ad hoc treatment to deal with the synchronization effect between client and server, that makes possible that a new request from the same client has to wait until the servers finishes the "post-service" phase for the previous request from itself. It was impossible, however, to use directly a $\mathrm{QN}$ solver for the solution of SRVN submodels (in the way [Rol88] does, for example), because the number of customers is variable due to second phases. Another problem that makes a QN solver inappropriate for SRVN is the fact that, in a QN, the custorsers are independent, while in SRVN there are strong synchronizations effects due to RV communication.

The second main modelling technique, $\mathrm{PN}$, has modelling abilities to represent concurrent software at almost any level of detail, from a very coarse to a very fine grain, but has a poor ability to model contention for resources. However, the main disadvantage of PNs is the lack of efficient approximate solution methods. The exact solution suffers from exponential 
explosion of the state space, and thus severely restricts the size of tractable models.

In conclusion, the bibliographical review has provided a conceptual framework for the solution of SRVN models, inspired in special from the literature on QN approximate solution methods, but also from the techniques at the Markov Chain level. The principles on which is based the new approximate solution for SRVN proposed in this thesis are:

- decomposition into submodels that are solved ite:=-tively

- Mean Value Analysis approach for the solution of a submodel, expressing the solmion in terms of mean throughputs, delays of the components, and arrival instant probabilitic's

- Markov Chain aggregation to obtain a set of simultaneous equations for the arrival instant probabilities. 


\section{Chapter 3}

\section{Markov Chain Task-Directed Aggregation}

\subsection{Formal Definition of an SRVN Model}

As shown in section 1.1.4, the Stochastic Rendezvous Network (SRVN) model has been introduced in [Wo086, Woo89] as a performance model for tasking systems with rendezvous (RV) type of communication. This section defines formally an SRVN model, and then reviews the principle of the heuristic approximate MVA solution algorithm for SRVN models proposed in [WNPM89].

An SRVN model containing a collection of communicating tasks is described by an acyclical communication graph that shows the pattern of interactions between tasks. A model with $N$ tasks is driven by a set of $R$ reference tasks (indexed $l$ to $R$ ) that do not accept RV requests, but rather cycle endlessly generating RV requests for the other tasks. An example wus given in Figure 1.4, where a tasking system with 9 tasks is driven by 6 reference tasks which cycle endlessly sending RV requests to other tasks, and waiting for a reply that marks the end of RV. The other three tasks are able to accept RV requests, and to "serve" them as follows:

: All RV requests for a server are queued in a single queue per server. The server starts its cycle with an $R V$ accept, taking the first request from the queue. If the queue is empty, the server is idle. After accepting a request, the server executes a first phase "in $\mathrm{RV}$ ", and then sends a reply to the client. 
- The client is released from RV by the reply and may resume. The server also continutes and executes an "after RV" part called second phase, that is concurrent with the client.

The class of SRVN considered here allows for different behaviours of a task depending on the entry point for which a request has arrived. An entry (the term is from Ada) may also be called a port or socket. Since a RV request from a client is sent to a specific server entry, we. will use the expression "a client requests an entry". The workload parameters characterizing the tasks are given by entry and phase as follows:

$s_{\text {iep }}=$ total execution time of task $i$, entry $e$ during phase $p$ (this does not include the waiting for RV requests made in this phase);

$y_{(i e p)(j d)}=$ mean number of RV requests made by task $i$, entry $e$, phase $p$ to task $j$, cutry $d$.

Figure 3.1 shows the state machine describing the behaviour of an accepting task $T_{1}$. The "idle state" is $e_{i 0}$. After accepting a request for an entry $e$, the task starts executing the first phase, then the second phase for the respective entry. Each phase is a secquence of identical exponentially distributed stochastic execution periods (when the task is in state $\left.u_{i e p}\right)$, separated by RV requests to other tasks. There are on average $Y_{\text {iep }}=\sum_{j} \sum_{d} y_{(i e p)(j d)}$ requests made by task $i$, entry $e$ in phase $p$, so that the mean duration of an execution period is $\mu_{\text {iep }}^{-1}=\frac{s_{\text {iep }}}{\left(Y_{\text {iep }}+1\right)}$. After every execution period the next event is selected randomly: rithers a RV request to task $j$ entry $d$ with probability $\frac{y_{(\text {tep })(2 d)}}{\left(Y_{\text {iep }}+1\right)}$, or end of phase with probability $\frac{1}{\left(Y_{\text {iep }}+1\right)}$. The queueing for RV is represented by state $q_{(i e p)(j d)}$, and the waiting for service by

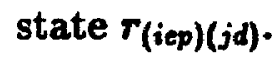

The state machine for a reference task is a particular case of Figure 3.1: there are IIO different entries and no idle state, due to the fact that such a task never accepts. For the same reason there is no "in RV" phase. All that remains is an execution state and a couple. of queueing and waiting for service states corresponding to the RV requests generated ly the respective task. Although a reference task does not have entry points for RV regurests, for notational consistency we consider that it has one entry with a "post RV" phase? (task $r$, entry 1, phase 2).

An SRVN task can represent either a software task or a hardware server ( $C P U, 1 / O$ device, etc.) A hardware server can be modelled by an SRVN task with "in RV" phase only, that 


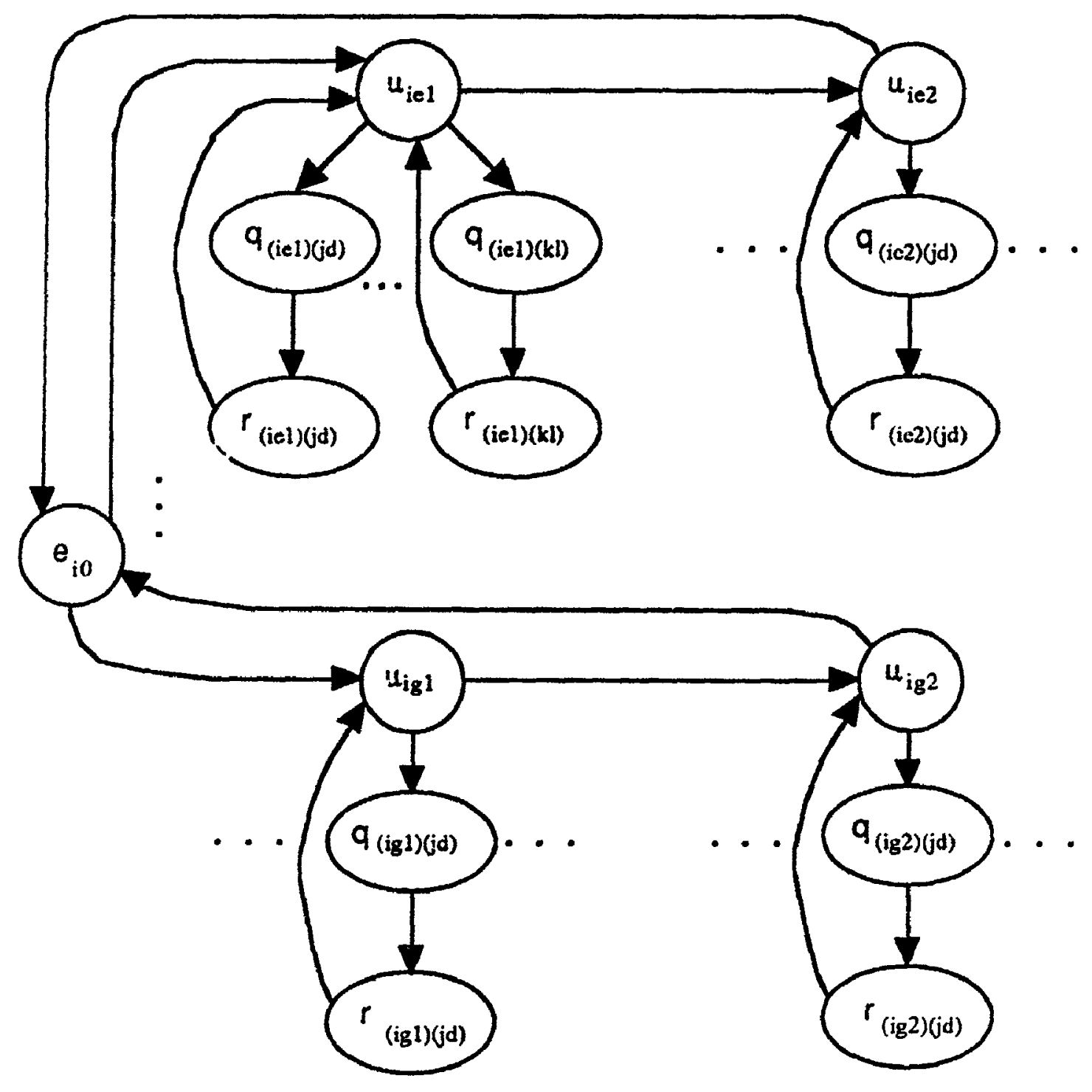

Figure 3.1: State machine for an accepting task $T_{i}$ with entries 
does not make any nested RV requests. It is implicitly assumed that the execution periods of a software task specified through its execution time parameters are done in parallel, as if the task had its own processor. However, we can model the processor contention by transferring to the processor the execution time of the software tasks coallocated on it, such that all "execution slice" of the task between two RV requests will give the service time for the processor, and the number of "slices" will give the number of RV requests from task to processor. Since each task phase may have a different execution "slice" between two RV: requests, the SRVN task representing the processor will have several entries, one for cach slice value. In [WNPM89] there is described an automatic procedure that extends an SRN N model by adding a task with multiple entries and first phase only to represent each shatril processor.

\subsubsection{Heuristic MVA solution method for SRVN}

The heuristic approximate MVA algorithm introduced in [Woo89] for SRVN witlout entries and in [WNPM89] for SRVN with entries solve iteratively a set of nonlinear equations relating task (entry) throughputs, mean duration of phases and mean waitings for queueing and for service of the RV requests. With the exception of the queueing delays that are determined by heuristic approximations (not shown here), the equations for the other quantities are exart, and they are summarized as follows.

The mean duration of phase $p$ of task $i$, entry $e$, including the waiting for RV requests, is given exactly by:

$$
x_{i e p}=s_{i e p}+\sum_{j} \sum_{d \in E(j)} y_{(i e p)(j d)}\left(w_{(i e p)(j d)}+x_{j d 1}\right)
$$

where $E(j)$ is the set of entries of task $j$, and $w_{(i e p)(j d)}$ is the queueing delay suffered by it RV request.

Reference task throughputs represent the main performance measures for the SRVN systems, from which other performance measure, as utilizations, can be obtained. 'Jhe' throughputs $f_{r}$ for reference task $r(r=1, \ldots R)$ are given by the following exact relat is m. $h_{1}$.

$$
f_{r}=\frac{1}{x_{r 12}}, \quad r=1, R
$$


The throughput $f_{j}$ of a non-reference task is given as the sum of its entry throughputs $\varphi_{j d}:$

$$
f_{j}=\sum_{d \in E(j)} \varphi_{j d}
$$

The entry throughput $\varphi_{j d}$ of a non-reference task $j$ is related to the throughputs of its clients by the synchronization imposed by RV, as shown in the following traffic equations:

$$
\varphi_{j d}=\sum_{i} \sum_{e \in E(i)} \sum_{p} y_{(i e p)(j d)} \varphi_{i e}, \quad j=R+1, N ; \quad d \in E(j)
$$

By solving the set of equations (3.4), the non-reference throughputs can be obtained directly as a linear combination of the reference throughputs, in the form:

$$
\varphi_{j d}=\sum_{r=1}^{R} a_{j d r} f_{r} \quad j=R+1, N
$$

The waiting $w_{(i e p)(j d)}$ is found by a heuristic approximation, analysing an open submodel comprising task $j$ and the effect of the rest of the system as follows:

- the stream of requests coming to $j$ from other tasks $i$ is assumed to be Poissonian, conditioned on $i$ not being already involved in phase 1 of an RV with $j$ (i.e. the submodel is assumed to have open arrivals)

- the delays due to RVs requested by task $j$, entry $d$, phase $p$ are incorporated in phase duration $x_{j d p}$ for all $p$, as given by (3.1).

Given that the RV requests are queued into a FIFO queue, the queueing time spent by a request from task $i$ to task $j$ is approximated in a way similar to that proposed in [Rei79] for FIFO queue, by determining the following components:

- delay due to the completion of the service being executed by $j$ when the request from $i$ arrives;

- delay due to other tasks queued at $j$ ahead of $i$.

In order to compute these delays, the probabilities of the following events are determined by heuristic approximation:

- event that a RV request from $i$ arriving at $j$ finds $j$ busy in phase $p$, serving task $k$; 
- event that a RV request from $i$ arriving at $j$ finds $k$ waiting in the queue.

The SRVN solution is obtained by an MVA-like algorithm based on two views that are applied iteratively to every task. In the first view, $w$ is considered to be known and $x$ is found by (3.1); in the second view $x$ is assumed known and $w$ is found by heuristic approximations. At every iteration, the reference throughputs are obtained by using (3.2), from which the other throughput are determined by (3.5) and (3.3).

A different heuristic approach for an approximate solution for the basic SRVN is take' $n$ in [NWMP87], where the RV queueing delay is obtained from a closed submodel built around

each accepting task. The representation of a client in the new algorithm proposed in chapter 4 is based on this approach.

As shown in section 1.1.4, a number of extensions have been proposed for SRVN models, modifying accordingly the solution algorithm. However, the solution principle presented in this section remains the same.

\section{Evaluation of the Heuristic Approach}

Practical experience with the heuristic SRVN algorithms shows that the errors for task throughputs are, usually, less than $10 \%$. However, in some cases the maximum error can rise to $15 \%$ or even $20 \%$. The causes for big errors are sometimes difficult to explain. One of the: cases with poor accuracy is for servers with unbalanced entries having a difference in service times of one order of magnitude or more. This is due to the fact that, for FIFO servers with very different service times for different clients, the $\mathrm{QN}$ based approaches do not work very well. (As mentioned in section 2.1.4, multi-class QN with FIFO servers having different service time per class are not product form).

The present research was motivated by two main factors:

- The need for a more rigorous theoretical foundation to replace the ad hoc collectisn of heuristic approximation used in the previous SRVN solutions;

- The search for SRVN solution methods with a better accuracy (average relative throughtput error under $5 \%$, and maximum error under $10 \%$ ). 


\subsection{Task-Directed Aggregation}

The new SRVN algorithm developed in this thesis decomposes an SRVN model into a number of interrelated submodels that are solved iteratively, similarly to the heuristic SRVN method discussed in the previous section. The new algorithm also uses the exact MVA relationships (3.2), (3.1), and the traffic equations (3.5) and (3.3). The differences are the following:

- the new algorithm solves closed submodels

- the arrival instant probabilities are determined from a set of simultaneous equations derived rigorously from a set of aggregated Markov Chains obtained by task-directed aggregation;

- the translation of results obtained for a submodel into parameters for other submodels is also based on task-directed aggregation.

The principle of task-directed aggregation is first defined and its properties analysed in the present section, and then it is applied to SRVN models in the following chapters.

\subsubsection{State Vector of a Tasking System}

A tasking system composed of a collection of single-threaded inter-communicating tasks can be modelled as a set of communicating state machines, as shown in section 1.1.1. The tasks may communicate synchronously (blocking send or rendezvous) or asynchronously (nonblocking send). A task can have periods of independent work (executing an activity), can be involved in communication with its peers by sending or receiving messages, and can require, wait for and use common resources. In order to give an uniform treatment to all system components, we consider that the resources are also modelled as finite state machines, and treat the signals for request, grant and release of resources as normal messages. In the following we understand by "task" any system component described by a finite state machine.

The state of the entire system is a vector $\sigma$ with $(n+m)$ components, containing the states of all tasks and all message queues:

$$
\sigma=\left(\sigma_{1} \ldots \sigma_{n} \sigma_{n+1} \ldots \sigma_{n+m}\right)
$$

where: 
$n$ is the number of tasks

$m$ is the number of message queues

$\sigma_{i},($ for $i=1, n)$ is the state of task $i$

$\sigma_{n+j},($ for $j=1, m)$ is the state of queue $j$

Task states may be classified into two categories: active and passive. A task in an active: state is executing one of its activities, while in a passive states it waits for some erent to happen. The passive state are of two types: idle state when a task is doing nothing but. waiting for an activation message, and waiting state when a task in the middle of doing some work has to wait for an event or a condition that will allow it to continue its work (for example: a resource to be free, a reply to come). The condition waited for is a part of the state description for the waiting state. A passive state ends when the task is awakened by an event due to another task (which obviously has to be active), or by an external event. The time spent by a task in an active state is described directly by model parameters, as opposed to the time spent in a passive state that is a result of the behaviour of the whole system.

Message queue states have to specify the number and type of messages waiting in the queue. The identity of the message sender is also important when the sender waits for an acknowledgement or a reply. Depending on the the queueing policy, the order in which the messages arrive at the queue may also be important. The queue states are passive, being similar to task waiting states.

Transitions from a system state to other system states are due to events marking the end of active task states. (If all elements of a system state are passive, then the state indicatess a deadlock). In Markovian systems, the events producing transitions are Poissonian.

If the communications between tasks are entirely synchronous as in the systems considered here, the system state can be completely specified by the subvector containing task states only:

$$
\sigma=\left(\sigma_{1} \sigma_{2} \ldots \sigma_{n}\right)
$$

This is true because the state of a queued message can be completely described by the wate of its sender, that is blocked waiting for a reply for the whole period the message stays in 
queue.

\subsubsection{TDA-1 and TDA-2: definitions and properties}

Task-directed aggregation (TDA) represents the theoretical foundation on which are based the new algorithms for SRVN models proposed in this thesis. TDA is the aggregation of a MC model for a tasking system with synchronous communication with the state vector as in (3.7); the argregation process is conducted in function of the states of some chosen task(s), named designated tasks. such that all MC staies containing the same values for the designated task(s) state are grouped together.

There are two types of task-direcied aggregation, TDA-1 and TDA-2, which will be used in different combinations for the derivation of the algorithms. TDA-1 aggregates across tasks, so the identity of non-designated tasks disappears in the new state vector. On the other hand, TDA-2 aggregates states within tasks.

Definition of TDA-1. All state vectors containing a given state subvector for the designated tasks are lumped together, disreg:rding the states of the non-designated task. Denote by $D$ the set of designated task indices, by $N$ the set of non-designated task indices, and by $\mathcal{S}_{i}$ the state set of task $i$. The system state vector can be written as $\sigma=\left(\sigma_{D} \sigma_{N}\right)$, where the subvector $\sigma_{D}$ contains the states $\sigma_{i}, i \in D$ of the designated tasks, and the subvector $\sigma_{N}$ contains the states $\sigma_{j}, j \in N$ of the non-designated tasks. By TDA-1 we obtain a new aggregated state vector $\sigma^{\prime}$ that indicates only designated task states, and is equal to $\sigma_{D}$ :

$$
\sigma^{\prime}=\bigcup_{\sigma_{i} \in S_{i}, i \in N}\left(\sigma_{D} \sigma_{N}\right)=\sigma_{D}
$$

Definition of TDA-2. The aggregation reduces the number of possible values for states of designated tasks, while keeping the same number of components in the state vector. Thus, every state set $\mathcal{S}_{i}, i \in D$ is replaced by a smaller set $\mathcal{S}_{i}^{*}$ containing aggregated task states $\sigma_{i}^{*}$. These are obtained by partitioning $\mathcal{S}_{i}$ into disjoint subsets $\zeta_{i}=\left\{\ldots, \sigma_{i}^{k}, \ldots\right\}$ and then aggregating the task states within subsets:

$$
\sigma_{i}^{*}=\bigcup_{\sigma_{i}^{k} \in \zeta_{i}} \sigma_{i}^{k}, \quad \text { for } \zeta_{i} \in \mathcal{S}_{i}, \quad i \in D
$$


As a result of TDA-2, the aggregated state vector $\sigma^{\prime \prime}$ has the form:

$$
\sigma^{\prime \prime}=\left(\sigma_{D}^{*} \sigma_{N}\right)
$$

where the subvector $\sigma_{D}^{*}$ contains states $\sigma_{i}^{*}, i \in D$, and the subvector $\sigma_{N}$ is unchanger.

\section{Properties of TDA-1}

As shown in subsection 3.2.1, the task states are divided into two classes: active and passive. The state vector $\sigma$ of the complete MC has an element for every task, a given system state being a combination of active and passive task state values. A system state has at least one outgoing transition for every active component, but none for the passive components. (A passive state change is always due to the change of an active state in someother task).

It is known that, in general, the aggregated transition rates of an aggregated M dopend on the probability vector $p(\sigma)$ of the complete MC . However, by applying THA-1 L, a complete MC for any set of designated tasks, the aggregated transition rates caue to all active state of a designated task have an independence property, as shown by the following lemma.

Lemma 1 Consider an aggregated MC obtained by TDA-1 from the complete MC: of a tosking system with $n$ tasks, where the designated task set $D$ contains a given task T, $(1 \leq|D|<n)$. Let $\Omega_{\alpha}$ be an aggregated state where $T_{i}$ is in an active state $\alpha_{i}$. If the duration of state $\alpha_{i}$ is exponentially distributed with rate $\mu_{i}$, then the sum of all aggregated rates $\lambda_{\alpha \beta}$ corres:ionding to all transitions leaving $\Omega_{\alpha}$ due to a change in the state of $T_{1}$ is independent of $M C$ probability vector, and equals $\mu_{i}$ :

$$
\sum_{\beta \neq \alpha} \lambda_{\alpha \beta \beta}=\mu_{i}
$$

As a particular case, if there is only one destination state $\Omega_{\beta}$ then: $\lambda_{\alpha \beta}=\mu_{i}$.

Proof. Figure 3.2 illustrates an aggregation obtained by TDA-1, with $T_{i}$ is one of the designated tasks.

Following the rules of $\mathrm{MC}$ aggregation, the transition rate $\lambda_{\alpha \beta}$ between two aggregated states $\Omega_{\alpha}$ and $\Omega_{\beta}$ is the weighted sum of the original rates $\lambda_{h g}$ for $h \in \Omega_{\alpha}$ and $g \in \Omega_{k j}$, 


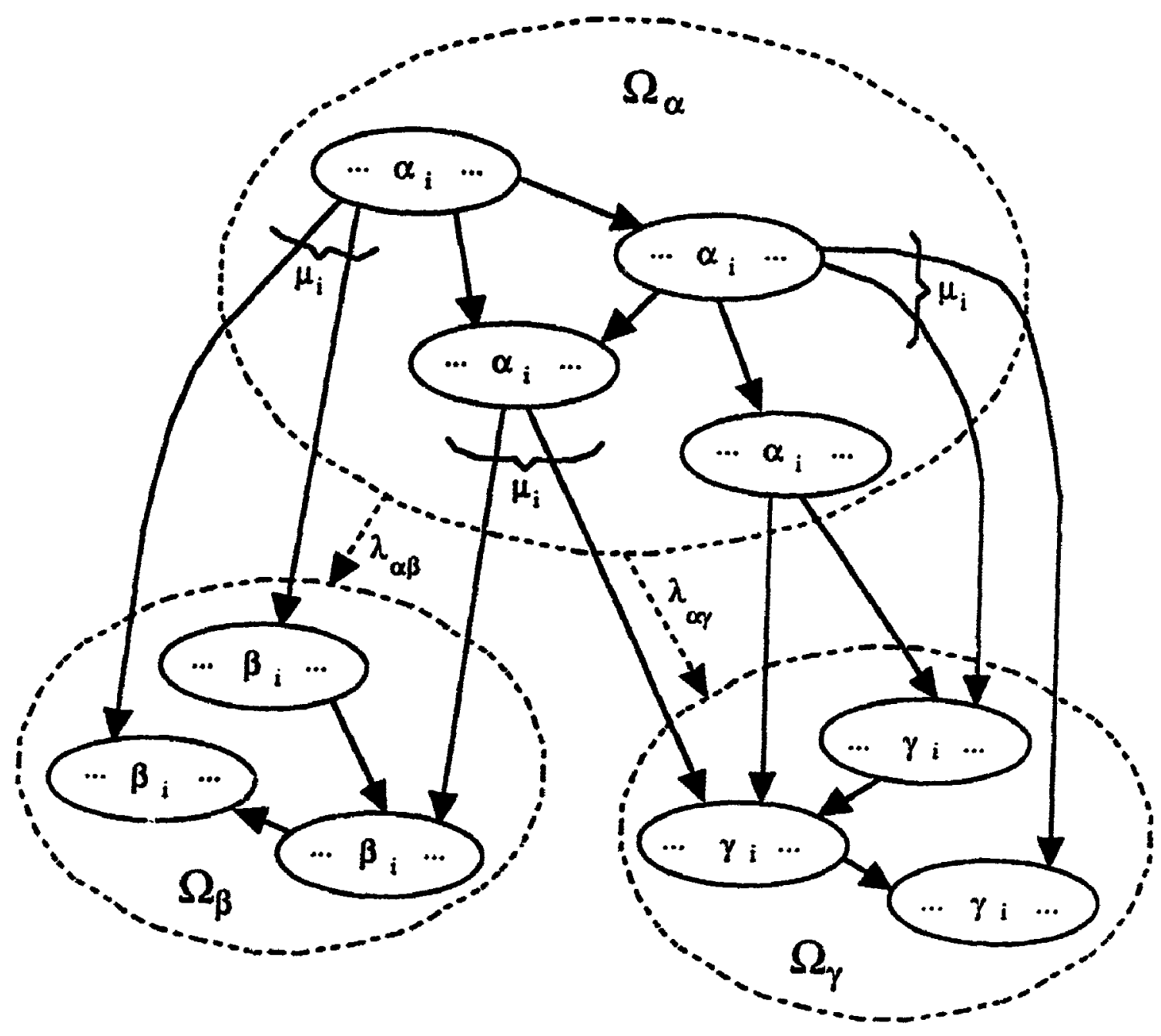

Figure 3.2: T'DA-1 aggregation where state $\alpha_{i}$ of $T_{i}$ is active $\left(T_{i} \in D\right.$, and $\left.|D|>1\right)$ 
conditioned on being in state $\Omega_{\alpha}$ :

$$
\lambda_{\alpha \beta}=\sum_{h \in \Omega_{a}} \sum_{g \in \Omega_{\rho}} p_{h} \lambda_{h g} / \sum_{h \in \Omega_{a}} p_{h}
$$

By the definition of TDA-1, every state $h \in \Omega_{\alpha}$ has the same subvector $\sigma_{D}$ with $\sigma_{i}=\alpha_{i}$. Any transition due to a designated task state change (meaning a change in $\sigma_{D}$ ) will leave $\boldsymbol{\Omega}_{\alpha}$. (The transitions inside the boundary of $\boldsymbol{\Omega}_{\alpha}$ are due exclusively to non-designated tasks).

Since every non-aggregated state $h \in \Omega_{\alpha}$ has one or more outgoing transitions due to a change of $T_{i}$ state with total rate $\mu_{i}$, and since all these transitions leave $\Omega_{\alpha}$ (crossing its boundaries), we can write:

$$
\sum_{\beta \neq \alpha} \sum_{g \in \Omega_{a}} \lambda_{h g}=\mu_{i v} \quad h \in \Omega_{\alpha}
$$

where the destination states $g$ belong to other aggregated states $\Omega_{\beta}, \beta \neq \alpha$.

By summing over all $h \in \Omega_{\alpha}$ and all aggregated destination states $\Omega_{\beta}$, and by using 3.12 and 3.13 we get:

$$
\sum_{\beta \neq \alpha} \lambda_{\alpha \beta}=\sum_{h \in \Omega_{\alpha}} \sum_{\beta \neq \alpha} \sum_{g \in \Omega_{\beta}} p_{h} \lambda_{h g} / \sum_{h \in \Omega_{\alpha}} p_{h}=\sum_{h \in \Omega_{\alpha}} p_{h} \mu_{i} / \sum_{h \in \Omega_{\alpha}} p_{h}=\mu_{i} \quad \text { QED }
$$

The particular case where there is only one destination state follows immediately.

Lemma 1 shows that the sum of outgoing transition rates due to an active state change: is independent of $\mathbf{p}(\sigma)$ (in the specified conditions), but does not say anything about the probabilities to choose between different destination states. In general, these choice probabilities depend on $p(\sigma)$, but there is a special case where they are independent, as shown by the following lemma.

Lemma 2 Let $T_{i}$ be a task with the state set $\mathcal{S}_{i}$, and $\alpha_{i} \in \mathcal{S}_{i}$ be an exponentially dis/ribuldrd active state with rate $\mu_{i}$. After executing $\alpha_{i}, T_{i}$ chooses with probability $\pi_{\alpha \beta \beta}$ to mont to a state $\beta_{i} \in \zeta_{i}$, where $\zeta_{i}$ is a given subset of $\mathcal{S}_{i}$, and $\sum_{\beta} \pi_{\alpha \beta}=1$. (All $\pi_{\alpha \beta}$ and $\mu_{i}$ are slate independent). Consider an aggregated MC obtained by TDA-1 from the complet: $M(:$, such that $T_{i}$ is one of the designated tasks, where $1 \leq|D|<n$. For every aggregated stalc: $\Omega_{\text {, }}$ in which $\sigma_{i}=\alpha_{i}$ there are exactly $\left|\zeta_{i}\right|$ groups of aggregated states $\Gamma_{\beta}=\left\{\ldots, \Omega_{\beta}, \ldots\right\}$. sucth that $\sigma_{i}=\beta_{i}$ for all $\Omega_{\beta} \in \Gamma_{\beta}$. (The difference between $\Omega_{\beta}$ states is due to $\sigma_{\text {, for another }}$ 
designated task $T_{j}, j \neq i$ ). The sum of aggregated transition rates $\lambda_{\alpha \beta}$ from $\Omega_{\alpha}$ to all $\Omega_{\beta} \in \Gamma_{\beta}$ is independent of MC probability vector, and equals $\pi_{\alpha \beta} \mu_{i}$.

$$
\sum_{\Omega_{\beta} \in \Gamma_{\beta}} \lambda_{\alpha \beta}=\pi_{\alpha \beta} \mu_{i}
$$

\section{Proof.}

Figure 3.3.a illustrates the state machine transitions of interest for $T_{i}$, and Figure 3.3.b the aggregated MC. By TDA-1 task $T_{i}$ is in state $\alpha_{i}$ for every state $h \in \Omega_{\alpha}$. For every state $h \in \Omega_{\alpha}$ there are at least $\left|\zeta_{i}\right|$ outgoing transitions due to the change of $T_{i}$ state from $\alpha_{i}$ to $\beta_{i} \in \zeta_{i}$, with rate $\lambda_{h g}$ such that:

$$
\sum_{\Omega_{\rho} \in \Gamma_{\beta}} \sum_{g \in \Omega_{\beta}} \lambda_{h g}=\pi_{\alpha \beta} \mu_{i}
$$

By summing over all $h \in \Omega_{\alpha}$ and all destination states, and by using the formula 3.12 for aggregated rates and 3.15 we get:

$$
\sum_{\boldsymbol{\Omega}_{\beta} \in \Gamma_{\beta}} \lambda_{\alpha \beta}=\sum h \in \Omega_{\alpha} \sum_{\boldsymbol{\Omega}_{\beta} \in \Gamma_{\beta}} \sum_{g \in S_{i}} p_{h} \lambda_{h g} / \sum_{h \in \Omega_{\alpha}} p_{h}=\sum_{h \in \Omega_{\alpha}} p_{h} \pi_{\alpha \beta} \mu_{i} / \sum_{h \in \Omega_{\alpha}} p_{h}=\pi_{\alpha \beta} \mu_{i}
$$

While lemma 1 and 2 refer to active task states, the following lemma is concerned with passive task states.

Lemma 3 Consider the aggregated MC obtained by TDA-1 from the complete MC of a tasking system with $n$ tasks, such that a given task $T_{i}$ is the unique designated task. Let $\Omega_{r}$ be the aggregated state where $T_{i}$ is in a passive state $\tau_{i}$. The mean sojourn time $d\left(\tau_{i}\right)$ spent by $T_{i}$ in the passive state $\tau_{i}$ equals the inverse of the sum of rates for all transitions leaving $\Omega_{\top}$ :

$$
d\left(\tau_{i}\right)=1 / \sum_{\beta \neq \tau} \lambda_{\tau \beta}
$$

Proof. Figure 3.4 illustrates an aggregation obtained by TDA-1 with $T_{i}$ as unique designated task. $\Omega_{r}$ contains all MC states $\sigma$ such that $\sigma_{i}=\tau_{i}$. The mean sojourn time $d_{h}$ of a state $h \in \Omega_{r}$ is given by:

$$
d_{h}=1 / \sum_{j=1}^{n} \mu_{j}
$$




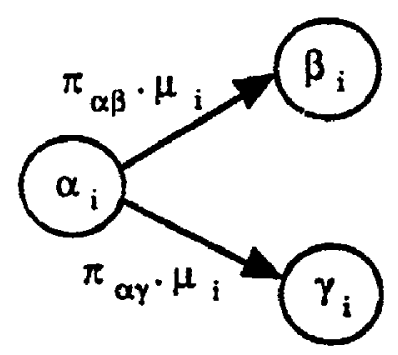

(a)
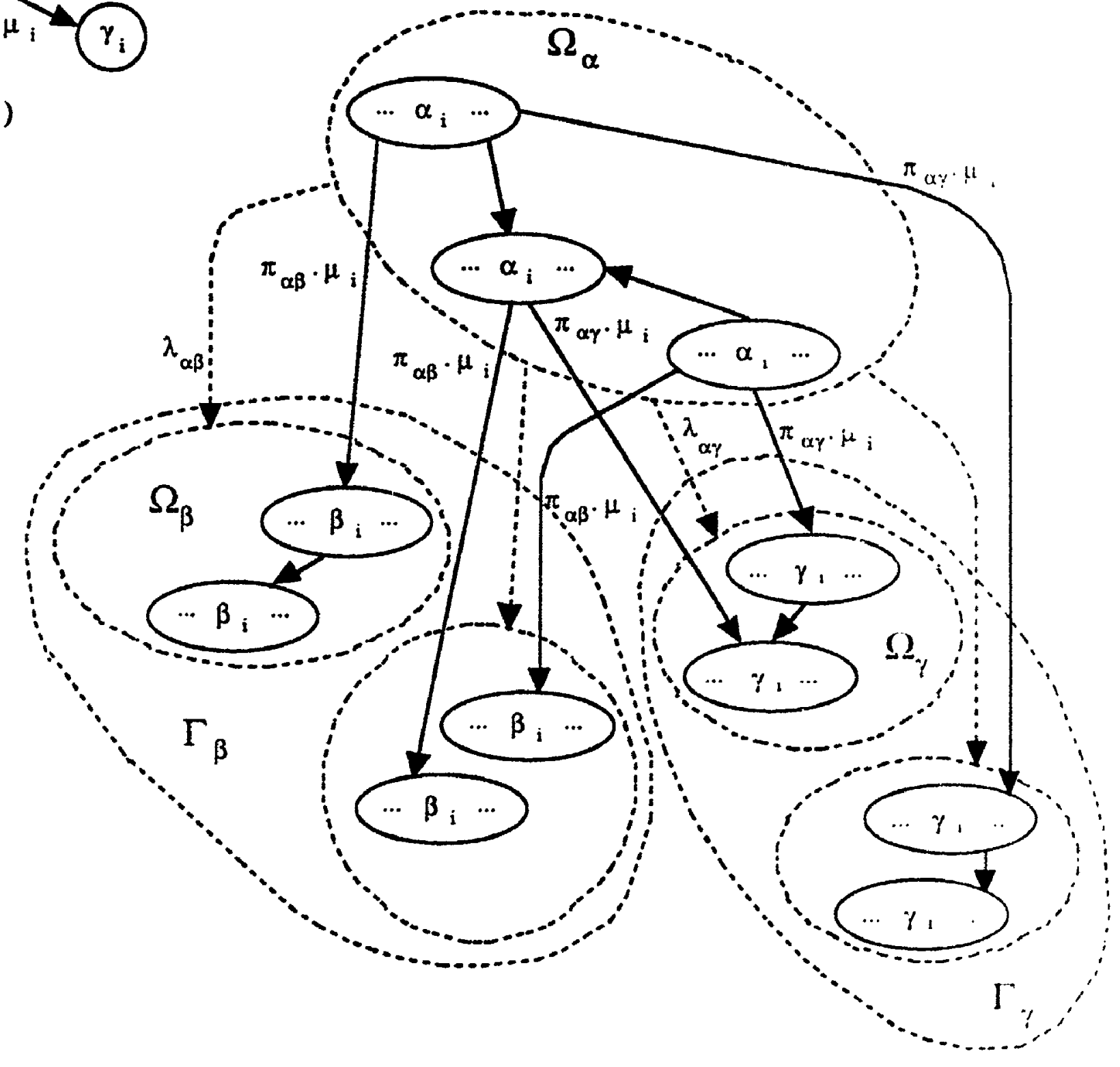

(b)

Figure 3.3: (a) Task state machine and (b) TDA-1 aggregation where $T$, in state $c_{1},+1, \ldots$ the next state with given probabilities $\left(T_{i} \in D\right.$, and $\left.|D|>1\right)$ 


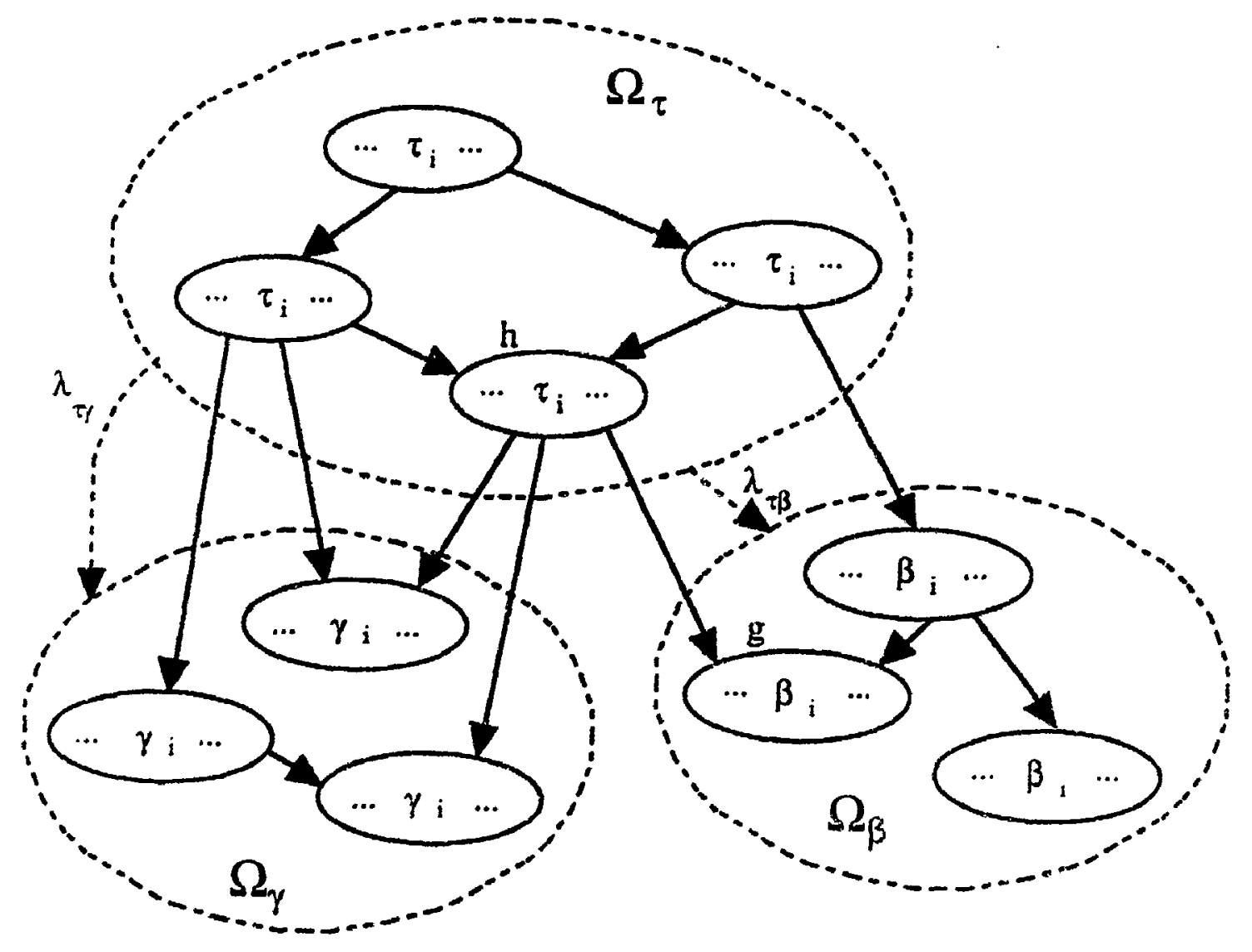

Figure 3.4: TDA-1 where state $\tau_{i}$ of $T_{i}$ is passive, and $T_{i}$ is the unique designated task 
where $\mu_{j}$ is the output rate for the $j$-th element of $\sigma$ (positive if $\sigma_{j}$ is active, and zero if $\sigma_{,}$ is passive) .

The mean sojuurn time $d\left(\tau_{i}\right)$ spent by $T_{i}$ in state $\tau_{i}$ is the weighted sum of $d_{h}$. for all $h \in \Omega_{r}$, obtained by Little's result applied first to the aggregated state $\Omega_{r}$, and then to every state $h \in \Omega_{\tau}$ :

$$
d\left(\tau_{i}\right)=\frac{\sum_{h \in \Omega_{r}} p_{h}}{\varphi\left(\tau_{i}\right)}=\frac{\sum_{h \in \Omega_{r}} \varphi_{h} d_{h}}{\varphi\left(\tau_{i}\right)}=\sum_{h \in \Omega_{\tau}} \frac{\varphi_{h}}{\varphi\left(\tau_{i}\right)} d_{h}
$$

where $\varphi_{h}$ and $\varphi\left(\tau_{i}\right)$ are throughputs of system state $h$ and task state $\tau_{i}$ respectively. The last. expression shows that the weighting probabilities for sojourn times are throughput ratios.

The transitions leaving $\Omega_{r}$ (meaning a change of $T_{i}$ from $\tau_{i}$ to some other state) are actually due to a change of active state for another task. Therefor the transition rates $\lambda_{h g}$ from $h \in \Omega_{\tau}$ to $g \in \Omega_{\beta}$ are different depending on $g$ and $h$. The sum of aggregated rat.'s for all transitions leaving state $\Omega_{\tau}$ is obtained by applying 3.12 and by summing over all destinations $\Omega_{\beta}$ :

$$
\sum_{\beta \neq \tau} \lambda_{\tau \beta}=\sum_{h \in \Omega_{\tau}} \sum_{\beta \neq \tau} \sum_{g \in \Omega_{\beta}} p_{h} \lambda_{h g} / \sum_{h \in \Omega_{\tau}} p_{h}
$$

The numerator represents the sum of throughputs for all transitions leaving state $\Omega_{r}$, which equals $\varphi\left(\tau_{i}\right)$. Therefore:

$$
\sum_{\beta \neq \tau} \lambda_{\tau \beta}=\varphi\left(r_{i}\right) / \sum_{h \subset \Omega_{r}} p_{h}
$$

3.16 results immediately from 3.18 and 3.19 .

By applying lemma 1 and 2 to active states and lemma 3 to passive states, we can determine immediately the mean performance measures for a task $T_{i}$ (i.e. mean state sojourn times, throughputs, state probabilities, etc.) from the aggregated MC obtained by TDA-1 with $T_{i}$ as unique designated task.

Consider now a case where the set of designated tasks $D$ contains more than one task. Let $\tau_{i}$ be a passive state of a task $i \in D$, and $\Omega_{\tau}$ the group of all MC states where $T_{2}$ is in state $\tau_{i}$ (as in lemma 3). By TDA-1 with $D>1$ we obtain several aggregated states $\Omega_{r}$ with different state subvertors $\sigma_{D}$ that have, however, the same value $\tau_{i}$ for the element $\sigma_{1}$. This means that all $\omega_{\tau}$ are subsets of $\Omega_{\tau}$, as shown in Figure 3.5. A similar partition takes place: for any other group of states $\Omega_{\beta}$. Due to the fact that the transition rate $\lambda_{h g}$ from a state $h \in \omega_{\tau}$ to $g \in \omega_{\beta}$ is dependent on the state vector $\sigma$ (as shown in the proof for lemma 3), 


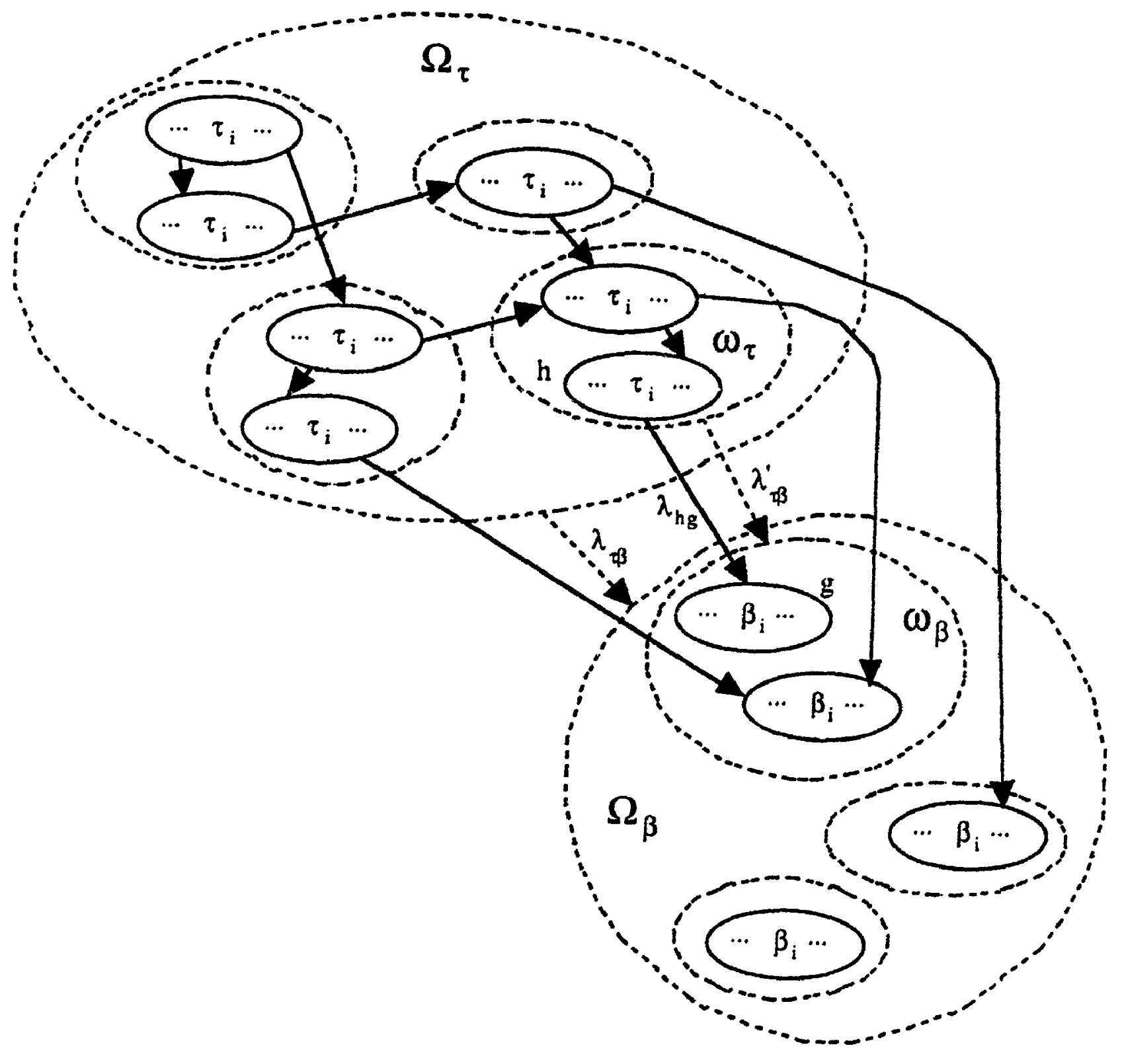

Figure 3.5: TDA-1 aggregation where state $\tau_{i}$ of $T_{i}$ is passive $\left(T_{i} \in D\right.$, and $\left.|D|>1\right)$ 
the aggregated transition rate $\lambda_{\tau \beta}^{\prime}$ from $\omega_{\tau}$ to $\omega_{\beta}$ is also dependent on $\sigma$. Also, the sojourn time of a state $\omega_{\tau}$ is dependent on $\sigma$.

A solution by decomposition for a tasking system assumes that, for a given sulmodel, the transition rates for passive states corresponding to interactions with the rest of the system are known from the results of other submodels. Although we have just shown liat liee rates for passive states are state dependent, we will approximate them by state inde'pe'nclent surrogate rates determined from mean sojourn times (as in lemma 3) that are import col from other submodels:

$$
\sum_{\beta \neq \tau} \lambda_{\tau \beta}^{\prime}=\sum_{\beta \neq \tau} \lambda_{\tau \beta}=1 / d\left(\tau_{i}\right)
$$

where:

$\lambda_{\tau \beta}^{\prime}$ is the transition rate from $\omega_{\tau}$ to $\omega_{\beta}$;

$\lambda_{\tau \beta}$ is the transition rate from $\Omega_{\tau}$ to $\Omega_{\beta}$;

$d\left(\tau_{i}\right)$ is the mean sojourn time spent by $T_{i}$ in state $\tau_{i}$.

This approximation is treating in fact external passive states as active states, and is inspired from the independence property given in lemma 1 for active states.

\section{Properties of TDA-2}

TDA-2 aggregates states at task level to represent tasks in a more abstract way, in order to reduce the complexity of MCs and the cost of their solution. Figure 3.6.a shows a state machine fragment for a task $T_{i}$ where a set of active states $\tau_{i}^{1}, \ldots, \tau_{i}^{m}$ are aggregated into a state $\tau_{i}^{*}$ :

$$
\tau_{i}^{*}=\bigcup_{u=1}^{m} \tau_{i}^{u}
$$

Let assume that there is a transition with rate $\mu_{i}^{u}$ from any state $\tau_{i}^{u}$ to another state $\beta_{i}$ such that $\beta_{i} \neq \tau_{i}^{v}$ for any $v$.

Figure 3.6.b shows the effect at $M C$ level of the aggregation done by TDA.-2 at task level, where the MC itself is aggregated by TDA-1 with $T_{i}$ as unique designated task $(|D|=1)$. The states $\Omega_{\tau}^{u}$ (where $T_{i}$ is in state $\tau_{i}^{u}$ ) are aggregated into $\Omega_{\tau}^{\#}$ :

$$
\Omega_{\tau}^{*}=\bigcup_{u=1}^{m} \Omega_{\tau}^{u}
$$



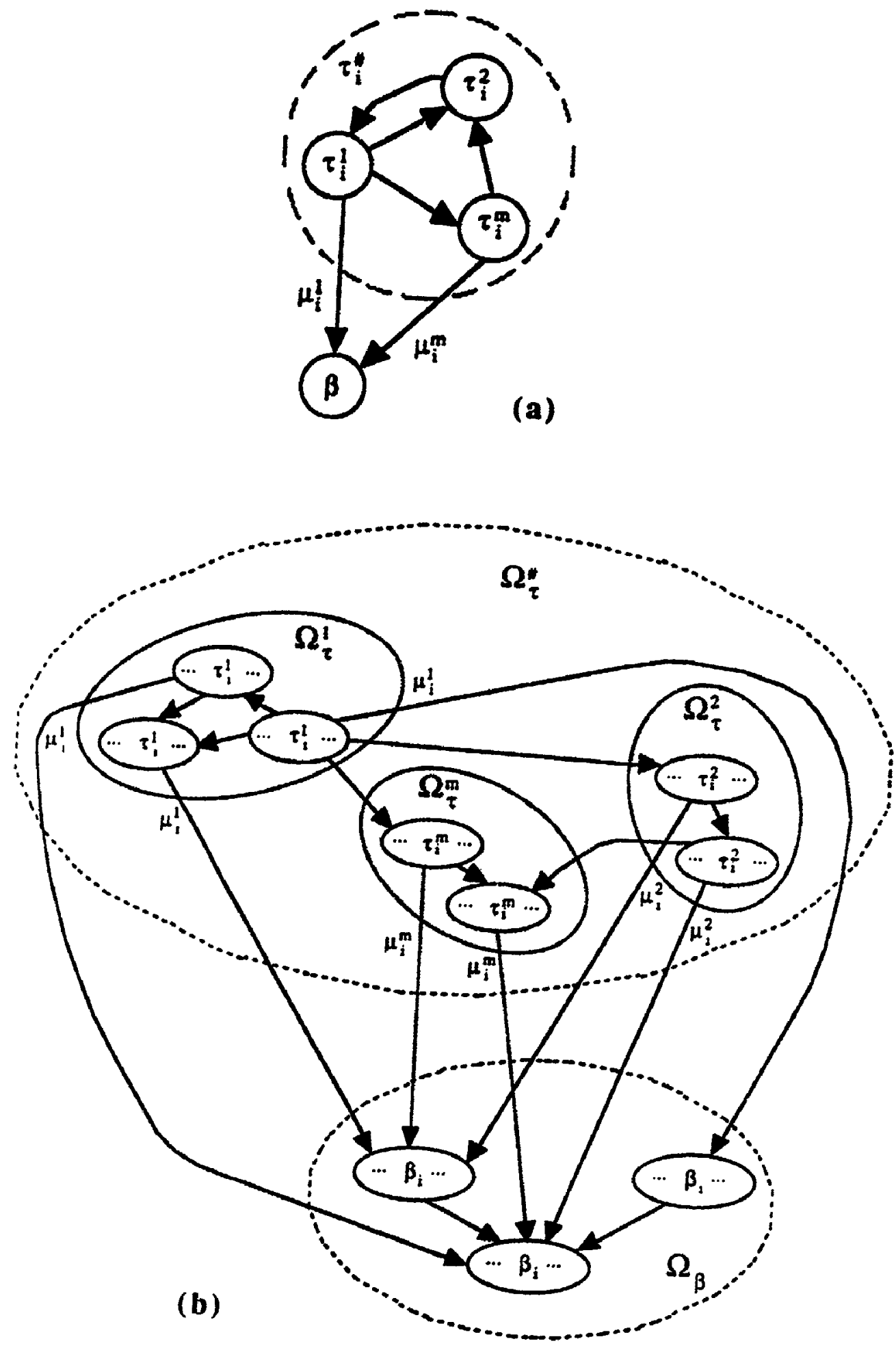

Figure 3.6: TDA-2 aggregation for $T_{i}$ : (a) at state machine level, and (b) at $\mathrm{MC}$ level, where the MC is aggregated by TDA-1 with $T_{i}$ as unique designated task 


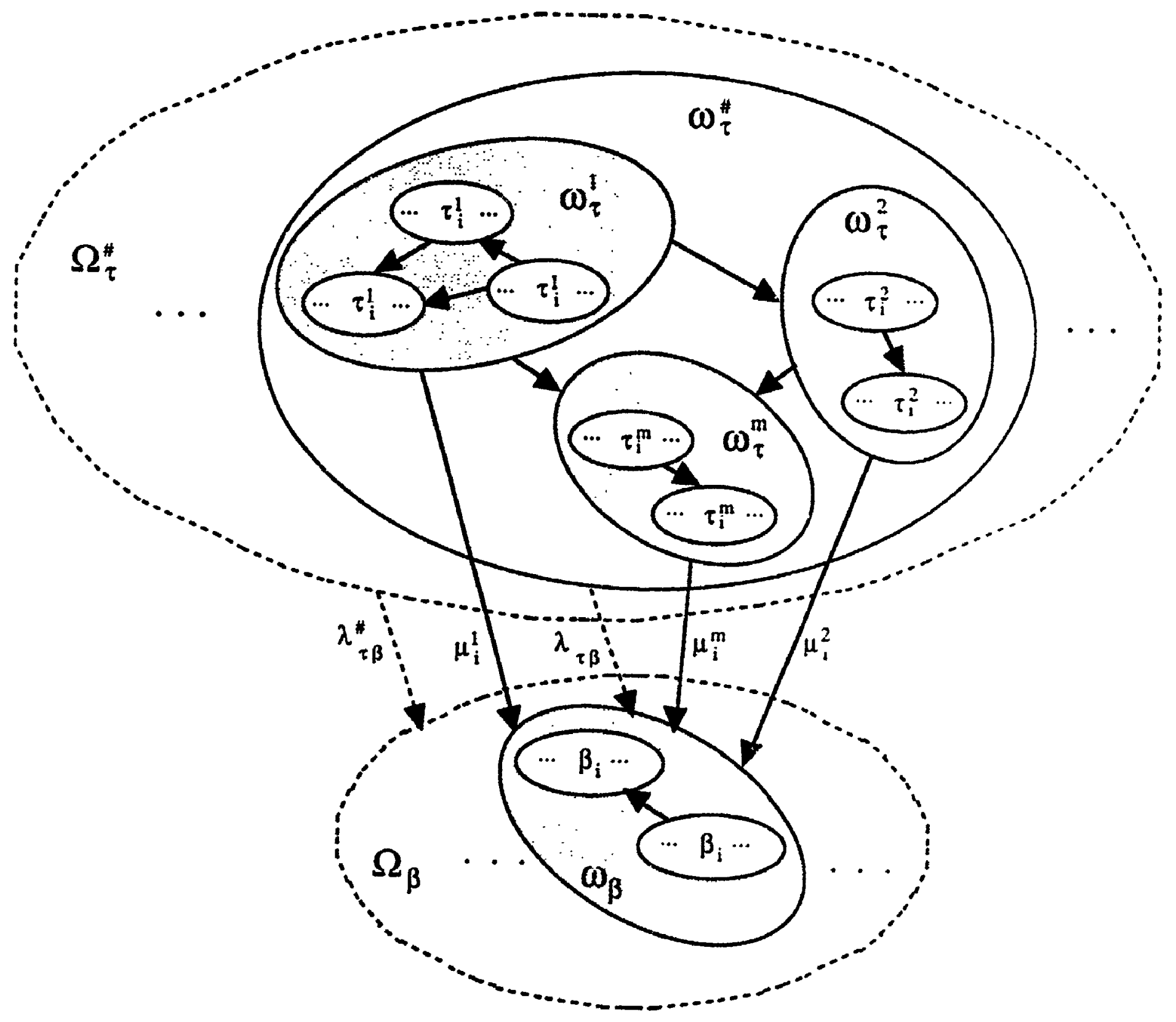

Figure 3.7: TDA-2 aggregation for $T_{i}$ : effect at $\mathrm{MC}$ level, where the $\mathrm{MC}$ is aggregated by TDA-1, with $T_{i} \in D$ and $|D|>1$ 
By lemma 1 , the aggregated transition rate from $\Omega_{\tau}^{u}$ to $\Omega_{\beta}$ equals the initial rate $\mu_{i}^{u}$. The aggregated rate $\lambda_{\tau \beta}^{*}$ from $\Omega_{\tau}^{*}$ to $\Omega_{\beta}$ is given by:

$$
\lambda_{\tau \beta}^{*}=\sum_{u=1}^{m} \mu_{i}^{u} p\left(\tau_{i}^{u}\right) / \sum_{u=1}^{m} p\left(\tau_{i}^{u}\right)
$$

where $p\left(\tau_{i}^{u}\right)=p\left(\Omega_{\tau}^{u}\right)$ is the probability of $T_{i}$ being in state $\tau_{i}^{u}$.

Consider now the aggregated MC obtained by TDA-1 for $|D|>1$ (see Figure 3.7). Every $\Omega_{\tau}^{u}$ from the previous figure is divided now into several aggregated states $\omega_{\tau}^{u}$ with different state subvectors $\sigma_{D}$ that differ by elements $\sigma_{j}$ for designated tasks $j \neq i$, but have the same value $\sigma_{i}=\tau_{i}$. The aggregation 3.21 by TDA-2 at task level has as effect the following aggregation at $\mathrm{MC}$ level:

$$
\omega_{\tau}^{*}=\bigcup_{u=1}^{m} \omega_{\tau}^{u}
$$

where the state subvectors $\sigma_{D}$ for all $\omega_{\tau}^{u}$ and $\omega_{\tau}^{*}$ differ only by the element $\sigma_{i}$. The aggregated rate $\xi_{\tau \beta}$ from $\omega_{r}^{*}$ to $\omega_{\beta}$ depends on the probabilities of $\omega_{\tau}^{u}$ as follows:

$$
\xi_{\tau \beta}=\sum_{u=1}^{m} \mu_{i}^{u} p\left(\omega_{\tau}^{u}\right) / \sum_{u=1}^{m} p\left(\omega_{\tau}^{u}\right)
$$

There are two possibilities to deal with state dependent rates as $\xi_{\ulcorner\beta}(\mathbf{p})$ :

- approximating it by a state independent rate, using the value obtained at task level:

$$
\xi_{\tau \beta}=\lambda_{\tau \beta}^{*} \text { for all } \omega_{\tau}^{*} \in \Omega_{\tau}^{*}
$$

- obtaining state dependent values by solving the MC by aggregation/disaggregation (using for example Takahashi's algorithm [Tak75] presented in the bibliographical review in section 2.2.1)

In the first case, TDA-2 offers a tradeoff of complexity against accuracy, by reducing the size of the MC at the price of getting less accurate results. In the second case, TDA-2 gives a criterion for state aggregation; the MC size is not affected, but the computation cost is reduced by solving several smaller MCs instead of a single big one.

TDA-2 can also be applied to aggregate passive states, in which case the mean performance measures obtained as results will be less detailed (and less accurate) than in the non-aggregated case.

In conclusion, TDA-2 can simplify the solution, but introduces appro:-imations with a negative impact on accuracy, that have to be estimated from case to case. 


\subsubsection{Task system decomposition based on TDA}

The state vector of a submodel represents only the states of its tasks, ignoring the rest of the system. The MC of a submodel looks like the result of TDA-1 applied to the complete MC, where the set of designated tasks $D$ contains all submodel tasks. However, in a solution where the decomposition takes place at the tasking system level, the MC of a submolld is not obtained by aggregation, but is rather built directly from components, as the Cartesian product of the state sets of its tasks. Due to the fact that some task states describing interaction with the rest of the system are approximate, it results that the MC for a sulmondel is also approximate. This approach avoids the construction and the solution of the MIC: for the whole system (that suffers from exponential explosion of the state space) at the price of decreasing the solution accuracy.

The task states within a submodel are of three types:

a) active states for independent execution;

b) external passive states for interactions with the rest of the system.

c) internal passive states for interactions inside the submodel.

The active states are described exactly by initial parameters of the model, the external passive states are approximated from the results of other submodels, while the performance measures obtained for the third class of states (throughputs and sojourn times) represent the results of the submodel.

In order to identify the points where approximations are made in the decomposition method, we compare the approximate MC of a submodel built directly from the states of its components with the exact MC that would be obtained from the complete MC of the system by TDA-1. The first observation is that both MCs have the same state vector $\sigma_{D}$ showing the designated task states (by the definition of TDA-1 in section 3.2.2), and thus they have the same state space. The transitions between states in both MCs are due to active and external passive task states.

Active states. By construction of the approximate $\mathrm{MC}$ and by applying lemma 1 to the exact MC, it results that the sum of outgoing transition rates due to the active task 
states $\sigma_{i} \in \sigma_{D}$ is the same for the corresponding states (with the same vector value) in both MCs.

If a task $T_{i}$ can choose a destination state $\beta_{i}$ with probability $\pi_{\alpha \beta}$ after the execution of an active state $\alpha_{i}$, (as shown in Figure 3.3.a), and if the choice probabilities are independent of the state vector, then the individual rates $\lambda_{\alpha \beta}$ for all $\beta$ are the same in both MCs (by lemma 2). If, on the other hand, the choice probabilities depend on the system state $\sigma=\left(\sigma_{D} \sigma_{N}\right)$ then it results that the individual rates $\lambda_{\alpha \beta}$ are also state dependent, even if the sum $\sum_{\theta} \lambda_{\alpha \beta}$ is state independent (by lem:.sa 1). Two cases can be distinguished:

- if the probabilities $\pi_{\alpha \beta}$ depend exclusively on $\sigma_{D}$ (only on designated task states), then the rates $\lambda_{\alpha \beta}$ depend on submodel states in a similar manner in both MCs;

- if the probabilities $\pi_{\alpha \beta}$ depend also on $\sigma_{N}$, then $\pi_{\alpha \beta}$ (and consequently $\lambda_{\alpha \beta}$ ) are different in the two MC, since the dependence on non-designated tasks is lost in the MC built directly from designated task states.

Passive states. The transition rates for external passive states (corresponding to interactions outside the submodel) are different in the two MCs. In the exact MC, the rates are state dependent as shown in subsection 3.2.2, depending on nondesignated tasks that are involved in the external interactions. In the approximate $\mathrm{MC}$, the state dependent rates are approximated by surrogate rates computed from the results of other submodels, that cannot capture the dependence on nondesignated tasks. A very straightforward approximation is to treat the external passive states as exponentially distributed active states with state independent rates determined, as in 3.20 , from mean sojourn times imported from other submodels.

In conclusion, the exact MC of a submodel (obtained by aggregation from the complete MC of the system) and the approximate MC (built directly from its components, with exact parameters for the active states, and approximate parameters for the external passive states) have exactly the same state space. However, their differencies are due to the dependency of some transition rates on nondesignated task states. This dependency is not captured in the second MC, that is built only from designated task states. 
An optional operation that can be conducted after identifying the classes of states for every task, with the purpose of reducing the solution complexity, is the aggregation of task states at task state machine level by TDA-2 (as shown in section 3.2.2). The aggregation must retain the distinction between the internal passive states giving the resulis of the submodel, and the other two classes of states that become the new "active" states of the. submodel, described by parameters or imported results. New aggregated surrogate rat $\cdots$; have to be determined at this stage for the new "active" states, either directly from the model parameters and imported results, or by using the surrogate rates determined previously.

In conclusion, a solution method by decomposition for a tasking system with syuchrumuns: communication will be obtained by following the steps:

A) Divide the model into submodels at tasking system level, such that every submotrl is solvable;

B) For each submodel

For each task

- identify external passive states corresponding to external interactions;

- treat them as active states with imported surrogate rates;

- apply TDA-2 at task state machine level, if possible;

- determine the aggregated surrogate rates for the new "active" states;

At the end of this step we know for every task of every submodel the set of (aggrcgated) states, and the formulas giving the transition rates for the "new active slutrs" as functions of model parameters and imported results.

C) For each submodel, build an approximate MC directly from the aggregated states of the submodel tasks, and obtain the balance equations or an equivalent system of equations that will be used in step D to solve the submodel;

D) Solve iteratively the system by solving each submodel in turn, (applying the mathod(s) from step C), and use at every iteration step new imported submodel parametcrs (obfained as in step B), until a desired precision is reached.

The first three steps are preliminary, their purpose being to guide the developmu'nt of an algorithm for the last step, that represents the actual iterative process producing the whition. 
This spproach can be applied to any tasking system with synchronous communication that can be described by a Markov chain. The algorithm(s) for submodel solution established in step $C$ may involve the construction and direct solution of a MC for every submodel, or may use approximate methods.

The rest of the thesis is organized as follows: the next two chapters will be dedicated to the development of two new algorithms for "client-server" SRVN submodels, first for clients with single-class requests, and then for clients with multi-class requests. Chapter 6 will follow the approach described above to propose a decomposition method for SRVN models, that will integrate into an iterative framework the new algorithms proposed in chapters 4 and 5. 


\section{Chapter 4}

\section{Arrival Instant Probabilities for Simple Client/Server SRVN}

\subsection{Introduction}

The goal of the present chapter is to develop an algorithm, called TDA/MVA(1), for the solution of a simple client/server SRVN presented in Figure 4.1, that contains a single server and a set of $n$ clients. The approach resembles the MVA corceptual framework for QNs, but has different arrival instant probabilities that are obtained by a rigorous analysis of the underlying MC model or simple client/server SRVN. The MVA solution technique for QNs (as reviewed in section 2.1.2) expresses the solution in terms of mean throughputs and delays of the components, using arrival instant probabilities, which are in turn expressed in terms of mean values for throughputs and delays. Although similar in spirit to queueing network MVA, the SRVN arrival instant probabilities are different. They are expressed by a set of simultaneous equations (4.41-4.48) in terms of mean values for throughputs and transition rates, and are derived in section 4.4 from the balance equations of a set of aggregated MCs obtained hy task-directed aggregation. Together with the other MVA equations for queuring delay, service time, and throughputs, they form a set of simultaneous nonlinear equations that, under the assumption of w. ponentially distributed execution times, give exact results for two clients, and approximate results for $n>2$ clients. A preliminary version of the algorithm was presented in [PWN89].

An SRVN client/server model, shown in Figure $a, 1$, has $n$ client tasks $T_{i}, i=1, n$ and one server task $T_{s .}$ In this chapter, we make the simplifying assumplion that a client may 

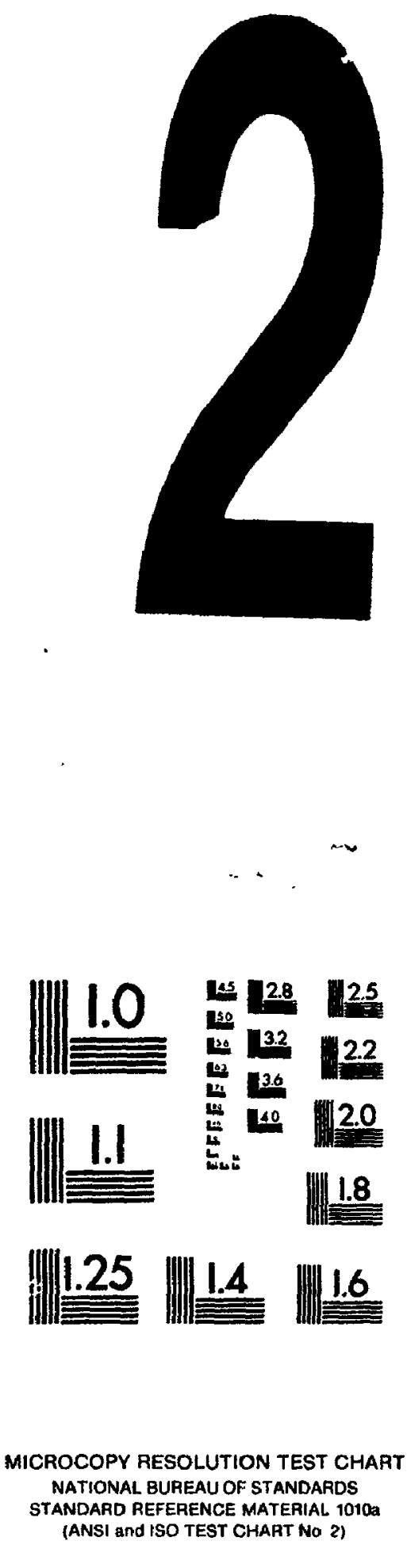


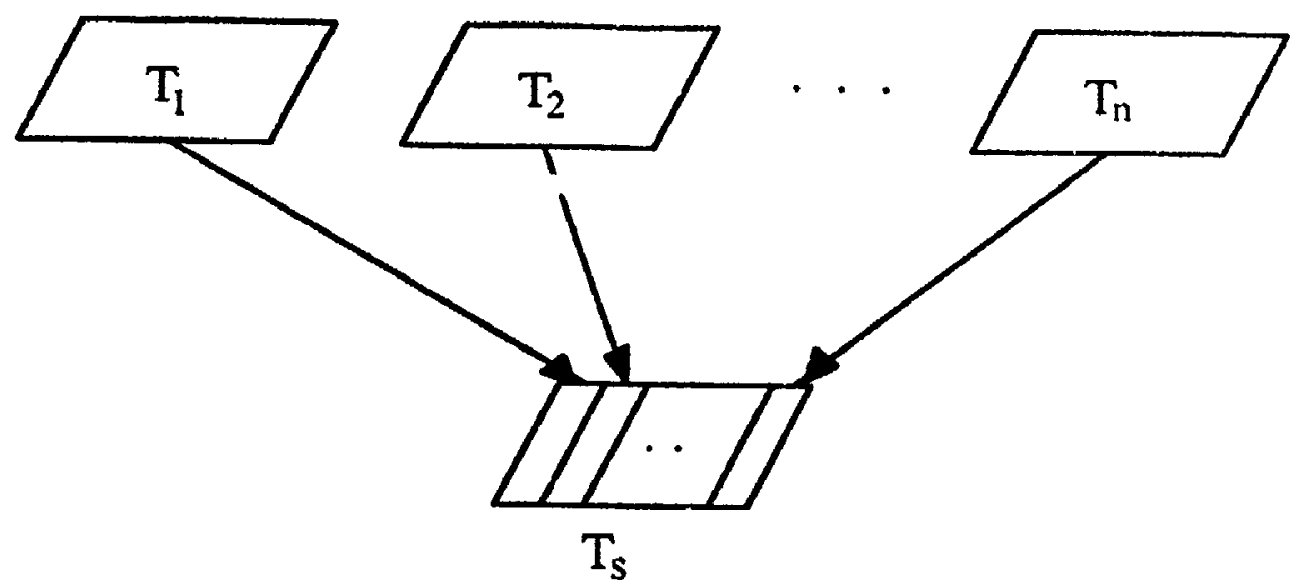

Figure 4.1: Simple client/server SRVN with $n$ clients and one server

request only one entry of the server. This restriction will be removed in chapter 5 . In order to simplify the notation, we assume here that the server has exactly $n$ entries, one for every client, such that entry $i$ is receiving RV requests only from client $T_{i}$. Each entry $k$ of $T_{\text {s }}$ has two phases ("in RV" and "after RV") that are assumed to have exponentially distributed service times $x_{s k 1}$ and $x_{s k 2}$ with given rates $\mu_{s k 1}$ and $\mu_{s k 2}$, respectively. A client $T_{i}$ is a task that never receives, but only sends RV requests to $T_{s}$ with frequency $F_{i s}$. The execution period of $T_{i}$ between a RV reply and the next $R V$ request is assumed to be exponentially distributed with rate $\xi_{i s}$. It is easy to see that a client has no "in RV" phase.

The present chapter is organized as follows. The Markov Chain of a simple client/server SRVN is studied in section 4.2, showing that its size grows exponentially with the number of clients $n$. Then, the "task-directed aggregation" technique is applied to the $\mathrm{MC}$ of a simple client/server SRVN in section 4.3, proving that the size of the aggregated MC grows linearly with the number of clients $n$, and that transition rate submatrices of the aggregated MCs have the same form for any number of clients $n$. Based on these results, the equations for arrival instant probabilities are obtained in section 4.4. The algorithm TDA/MV $(1)$ is presented in section 4.5, followed by assessment of its accuracy and convergence through experimental results. 


\subsection{Markov Chain model of a simple client/server SRVN}

\subsubsection{MC of a simple client/server SRVN with two clients}

In the present section we consider the simple case of a client/server SRVN model with a server $T$, and $n=2$ clients, that are denoted for generality by $T_{i}$ and $T_{j}(i=1$ and $j=2)$. The state vector $\sigma=\left(\sigma_{i} \sigma_{j} \sigma_{s}\right)$ has three components, one for every task.

The set of states $\mathcal{S}_{k}$ of a client $T_{k}$ is:

$$
\mathcal{S}_{k}=\left\{a_{k s}, r_{k s}, q_{k s}^{(m)}\right\} \quad \text { for } m=1, n
$$

where:

$a_{k s}=$ executing independently (exponentially distributed with rate $\xi_{k s}$ )

$r_{k s}=$ being served "in RV" phase by the server

$q_{k s}^{(m)}=$ waiting in queue at the server on the $m$-th position

When deriving some arrival instant probabilities, we want to emphasize the relative position in queue of a client $T_{i}$ with respect to a given competitor $T_{j}$, in which case the notation $q_{i s}^{(a)}$ is used for " $T_{i}$ ahead of $T_{j}$ ", and $q_{i s}^{(b)}$ for " $T_{i}$ behind $T_{j}$ ", respectively. For the case with two clients, $q_{i s}^{(a)}$ is equivalent to $q_{i s}^{(1)}$, and $q_{i s}^{(b)}$ to $q_{i s}^{(2)}$.

The set of states $\mathcal{S}_{s}$ for the server is:

$$
\mathcal{S}_{s}=\left\{e_{s 0}, e_{s 11}, \ldots, e_{s k p}, \ldots e_{s n 2}\right\}, \quad \text { for } k=1, n ; p=1,2
$$

where.

$e_{s 0}=$ idle server

$e_{s k p}=$ executing entry $k$, phase $p$ (exponentially distributed with rate $\mu_{s k p}$ ).

Figure 4.2 shows the complete MC model in continuous time for a client/server SRVN with two clients and a server. The transitions between states are due to three types of events: arrival of a request from $T_{i}$ (drawn in solid heavy lines), arrival of a request from $T_{j}$ (in heavy dashed lines), and end of server phases (in thin lines). 


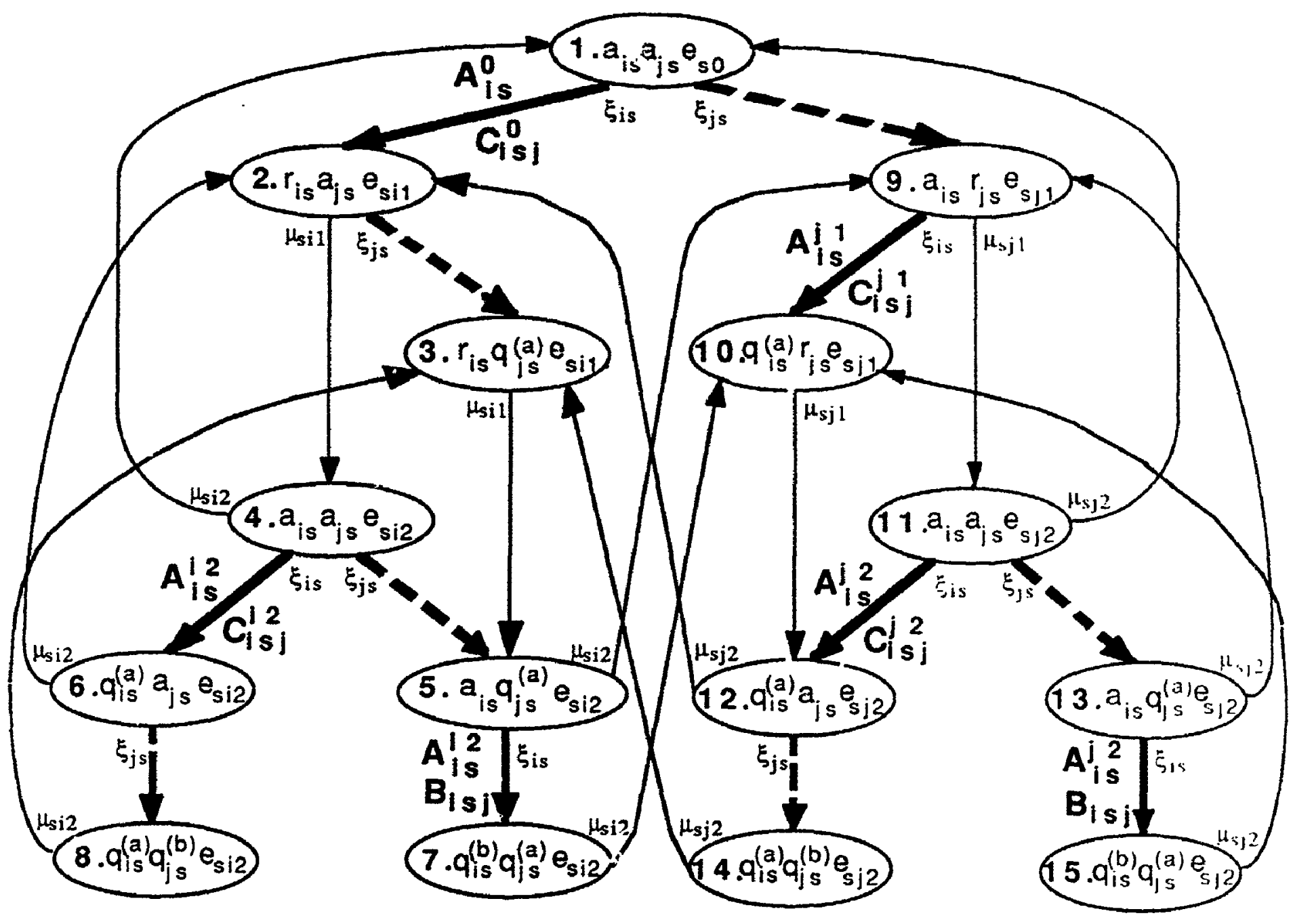

Figure 4.2: Markov Chain for a simple client/server SRVN with two clients 


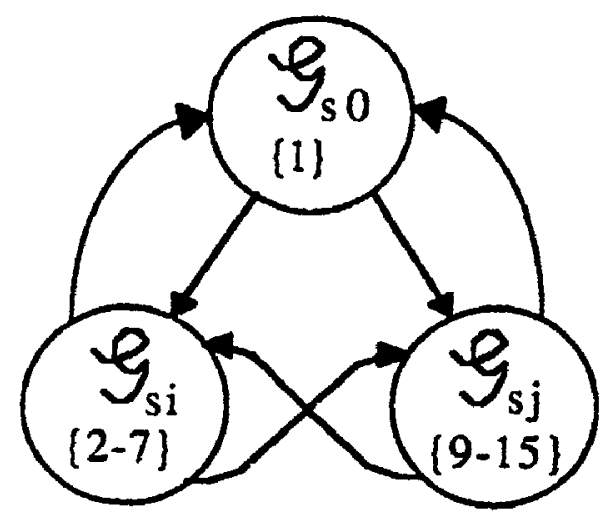

Figure 4.3: Groups of states of the MC for a simple client/server SRVN with two clients

We will find it useful to see that the MC of Figure 4.2 can be aggregated into the MC of Figure 4.3, by identifying three groups of states. Group $\mathcal{G}_{s 0}=\{1\}$ contains the state in which the server is idle, $\mathcal{G}_{s i}=\{2, \ldots, 8\}$ contains states in which $T$, is executing entry $i$, and $\mathcal{G}_{s,}=\{9, \ldots, 15\}$ contains states in which $T_{s}$ is executing entry $j$.

The transition rate matrix of the continuous time MC from Figure 4.2 for the simple client/server SRVN with 2 clients has the following form:

$$
\mathbf{Q}(2)=\left|\begin{array}{lll}
\mathbf{G}_{00} & \mathbf{H}_{01} & \mathbf{H}_{02} \\
\mathbf{H}_{10} & \mathbf{G}_{11} & \mathbf{H}_{12} \\
\mathbf{H}_{20} & \mathbf{H}_{21} & \mathbf{G}_{22}
\end{array}\right|
$$

where:

$\mathbf{G}_{\mathbf{i i}}$ is a diagonal submatrix whose nondiagonal elements represent transition rates between states inside group $\mathcal{G}_{s i}$, and whose diagonal elements are the sums (with negative sign) of all the rates for changing from the current state to any other state

$\mathbf{H}_{\mathbf{i j}}$ is a nondiagonal submatrix containing transition rates from states in $\mathcal{G}_{s i}$ to states in $\mathcal{G}_{s j} \cdot$

A submatrix $G_{i i}$ of size $(7 \times 7)$ has the form: 


$$
\mathbf{G}_{\mathrm{ii}}=\left|\begin{array}{ccccccc}
-\xi_{j,}-\mu_{s i 2} & \xi_{j 2} & \mu_{s i 1} & 0 & 0 & 0 & 0 \\
0 & -\mu_{z i 1} & 0 & \mu_{z i 1} & 0 & 0 & 0 \\
0 & 0 & -\xi_{i 2}-\xi_{j s}-\mu_{s i 2} & \xi_{j,} & \xi_{i,} & 0 & 0 \\
0 & 0 & 0 & -\xi_{i s}-\mu_{z i 2} & 0 & \xi_{i s} & 0 \\
\mu_{z i 2} & 0 & 0 & 0 & -\xi_{j,}-\mu_{s i 2} & 0 & \xi_{j,} \\
0 & 0 & 0 & 0 & 0 & -\mu_{s i 2} & 0 \\
0 & \mu_{z i 2} & 0 & 0 & 0 & 0 & -\mu_{s i 2}
\end{array}\right|
$$

Submatrix $G_{00}$ of size $(1 \times 1)$ has only one element with value $-\sum_{i=1}^{2} \xi_{i s}$.

Submatrices $\mathbf{H}_{\mathbf{i j}}$ are sparse, containing at most one non-null element per row. The'y have: different forms as follows:

$$
\begin{aligned}
& \mathbf{H}_{\mathbf{i j}}=\left|\begin{array}{ccccccc}
0 & 0 & 0 & 0 & 0 & 0 & 0 \\
0 & 0 & 0 & 0 & 0 & 0 & 0 \\
0 & 0 & 0 & 0 & 0 & 0 & 0 \\
\mu_{s i 2} & 0 & 0 & 0 & 0 & 0 & 0 \\
0 & 0 & 0 & 0 & 0 & 0 & 0 \\
0 & \mu_{s i 2} & 0 & 0 & 0 & 0 & 0 \\
0 & 0 & 0 & 0 & 0 & 0 & 0
\end{array}\right| \quad \text { for } i, j>0 \\
& \mathbf{H}_{\mathbf{i 0}}=\left|\begin{array}{c}
0 \\
0 \\
\mu_{\text {si2 }} \\
0 \\
0 \\
0 \\
0
\end{array}\right|
\end{aligned}
$$

\subsubsection{MC of a simple client/server SRVN with $n>2$ clients}

The state vector $\sigma=\left(\sigma_{1} \sigma_{2} \ldots \sigma_{n} \sigma_{s}\right)$ of a simple client/server SRVN with $n$ clients and onc server has $n+1$ components, as shown in section 3.2.1. The sets of task states are the same as in the previous section (see 4.1 and 4.2), with the observation that the number of client. and server states grows linearly with $n$. The server has two more states $e_{s k 1}, e_{s k 2}$ for every client $T_{k}$ added to the model, and a clieat $T_{i}$ has one more state $q_{i s}^{(m)}$ for every new pusition in the queue. The queue states are implicitly represented by task states, being thus scattercod over the whole vector $\sigma$. In order to emphasize the state of the queue, we introduce the following $\delta$-notation as a short hand for a state vector $\sigma$ where the server excentes entry $k$, phase $p$ :

$$
\delta_{s k p}^{Q}=\left(\sigma_{1} \ldots \sigma_{k} \ldots \sigma_{n} e_{s k p}\right)
$$




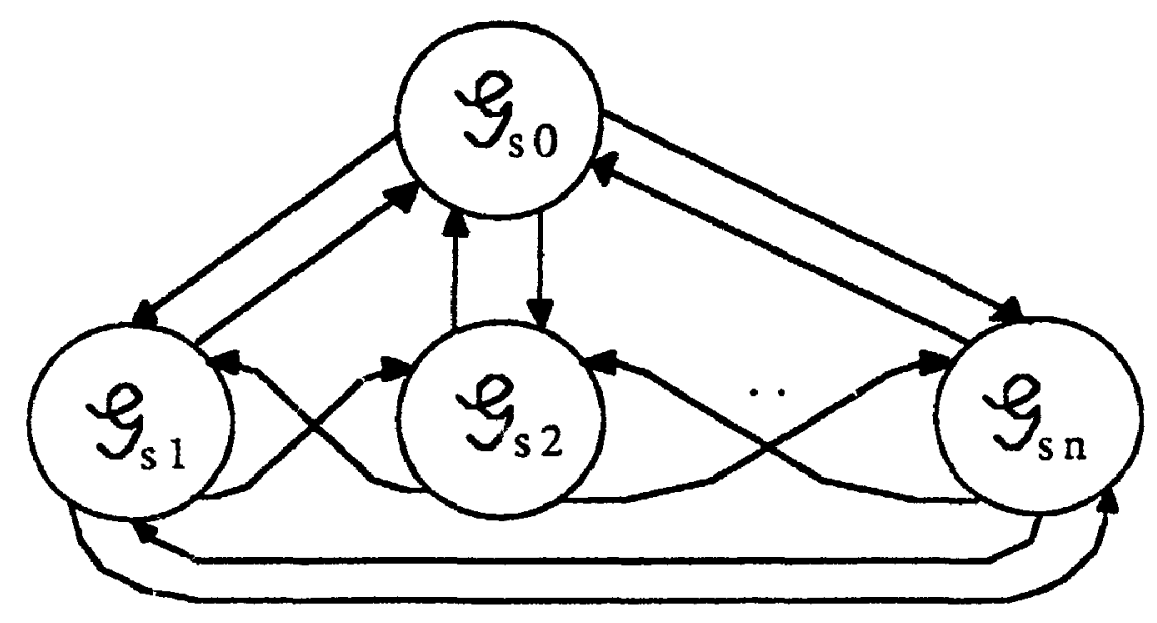

Figure 4.4: Groups of states of the MC for a simple client/server SRVN with $n$ clients

$Q$ is an ordered set of task indices containing the clients that are queued at the server in order of their arrival (this information is implicit in $\sigma_{1}, \ldots, \sigma_{n}$ ). There are two functions, first $(Q)$ and last $(Q)$, showing the first and the last task in queue, respectively.

While the state vector notation (that indicates explicitly all task states) is appropriate to describe the $\mathrm{MC}$ aggregation, the $\delta$-notation (that puts the emphasis on queue configuration and server state) is more appropriate to write the balance equations.

The state of the client in service $T_{k}$ takes one of the following values in state $\delta_{s k p}^{Q}$ :

$$
\sigma_{k}= \begin{cases}r_{k s} & \text { if } p=1 \\ a_{i s} & \text { if } p \neq 1 \text { and } k \notin Q \\ q_{i s}^{(m)} & \text { if } p \neq 1 \text { and } k \in Q\end{cases}
$$

The state $\sigma_{i}$ of a client $T_{i} \neq T_{k}$ takes one of the following values:

$$
\sigma_{i}= \begin{cases}a_{i s} & \text { if } i \notin Q \\ q_{i \varepsilon}^{(m)} & \text { if } i \in Q\end{cases}
$$

The notation for the "idle" state in which the server is idle and the queue is empty is:

$$
\delta_{s 0}^{\theta}=\left(a_{1} \ldots a_{k s} \ldots a_{n s} e_{s 0}\right)
$$

Just as the complete MC model for a system with $n=2$ clients shown in Figure 4.2 can be aggregated to Figure 4.3 by identifying three groups of states $\mathcal{G}_{s k}$ (for $k=0,2$ ), so can the complete MC model for $n>2$ be aggregated to the form in Figure 4.4 by identifying 
$n+1$ groups of states $\mathcal{G}_{\text {sk }}$ (for $k=0, n$ ). Group $\mathcal{G}_{\mathrm{so}}$ contains the idle state $\delta_{\mathrm{s} 0}(\mathcal{H})$ where the server is idle and the queue is empty, and a group $\mathcal{G}_{s k}$, for $k>0$ contains all state's in which the server is executing entry $k$. Figure 4.5 shows as an example the group $\vec{G}_{41}$ of $t_{14}$ MC for a simple client/server model with 3 clients.

Any group $\mathcal{G}_{\text {ok }}(k>0)$ can be divided into two subgroups $\mathcal{G}_{s k 1}$ and $\mathcal{G}_{s k 2}$, dipending (nn

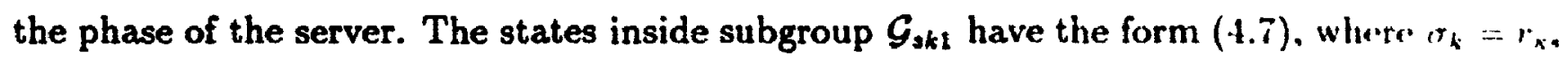
and $e_{s k p}=e_{s k 1}$. The queue length $|Q|$ varies between zero and $(n-1)$. The number of stitu's in $\mathcal{G}_{\text {ak1 }}$ having the queue length $|Q|=i$ equals $\frac{(n-1) !}{(n-i-1) !}$.

The total number of states $N_{1}$ in a group $\mathcal{G}_{s k 1}$ is given by:

$$
N_{1}=\sum_{i=0}^{n-1} \frac{(n-1) !}{(n-i-1) !}
$$

Subgroup $\mathcal{G}_{s k 2}$ corresponds to the second phase of service for $T_{k}$, containing states of the' form (4.7) where $\sigma_{k} \neq r_{i s,}$ and $e_{s k p}=e_{s k 2}$. The queue length varies between 0 and $n$. 'Th'rr" are $\frac{n !}{(n-i) !}$ states in $\mathcal{G}_{\text {sk2 }}$ with th: queue length $Q=i$.

The total number of states $N_{2}$ in subgroup $\mathcal{G}_{s k 2}$ is given by:

$$
N_{2}=1+n N_{1}=\sum_{i=0}^{n} \frac{n !}{(n-i) !}
$$

The size $M$ of a MC model for a simple client/server SRVN with $n$ clients grows exponentially with $n$, as shown in the following expression:

$$
M=1+n\left(N_{1}+N_{2}\right)=\sum_{i=0}^{n} \frac{(n+1) !}{(n-i) !}
$$

Table 4.1 gives the values for $N_{1}, N_{2}$ and $M$ for up to 6 clients.

The balance equations for the MC model of a client/server SRVN with $n$ clients are giv' $\|$ by (4.12)-(4.17).

$$
\begin{aligned}
& \mathcal{P}\left(\delta_{s k 1}^{Q}\right)\left(\sum_{i \notin Q} \xi_{i \varepsilon}+\mu_{s k 1}\right)=\mathcal{P}\left(\delta_{s k 1}^{Q-\{l\}}\right) \xi_{l,}+\sum_{i=1}^{n} \mathcal{P}\left(\delta_{s i 2}^{\{k\}+Q}\right) \mu_{s i 2} \\
& \text { where } l=\operatorname{last}(Q), \quad k=\operatorname{first}(\{k\}+Q) \\
& \mathcal{P}\left(\delta_{s k 1}^{s}\right)\left(\sum_{i=1}^{n} \xi_{i s}+\mu_{s k 1}\right)=\mathcal{P}\left(\delta_{s 0}^{\bullet}\right) \xi_{k s}+\sum_{i=1}^{n} \mathcal{P}\left(\delta_{s 12}^{\{k\}}\right) \mu_{s i 2}
\end{aligned}
$$




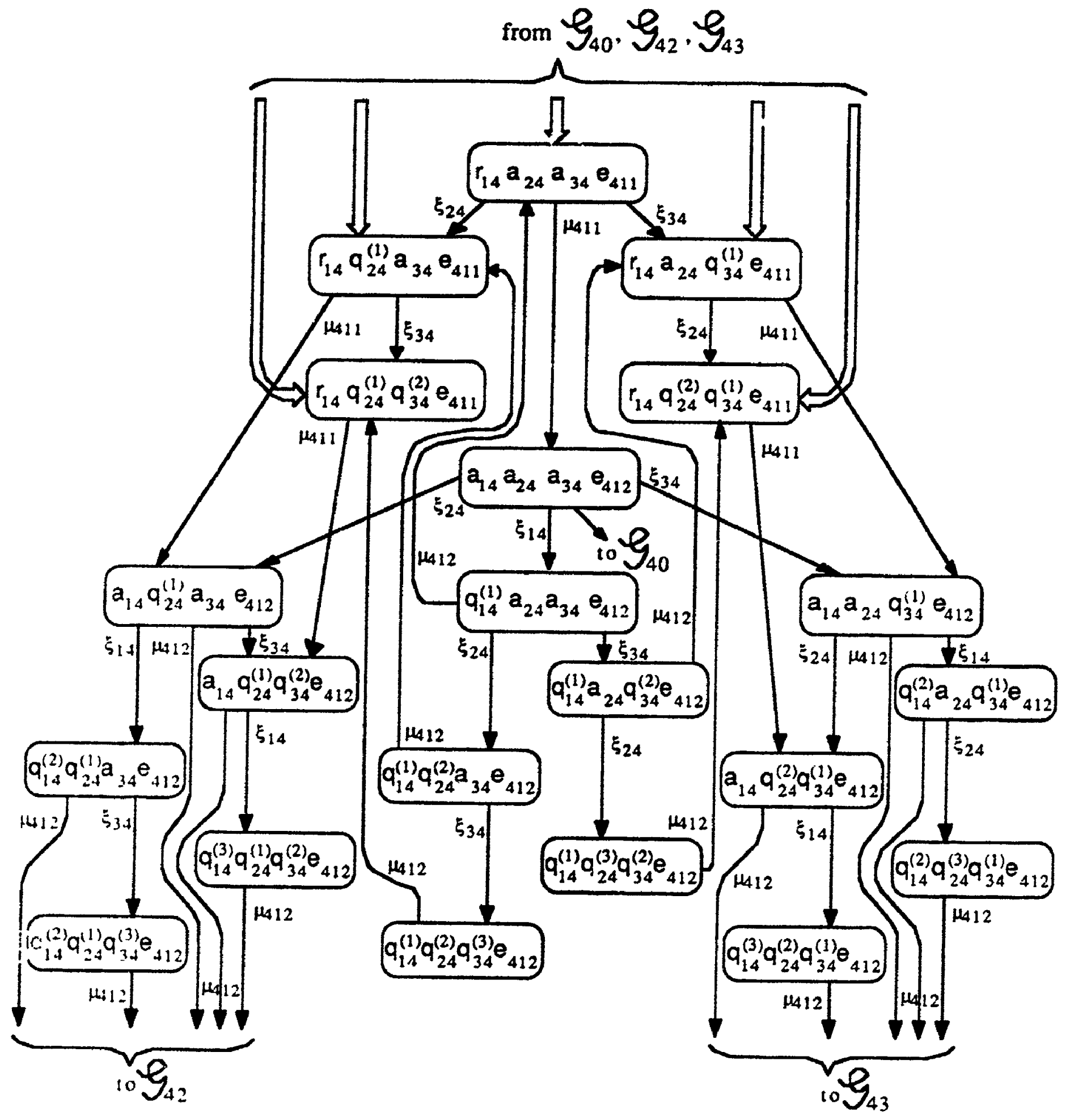

Figure 4.5: Group $\mathcal{G}_{41}$ of the MC for a simple client/server SRVN with 3 clients 
Table 4.1: Markov Chain size of a client/server model

\begin{tabular}{||c|rrr||}
\hline Number of clients & \multicolumn{3}{|c||}{ Number of states } \\
$n$ & $N_{1}$ & $N_{2}$ & MI \\
\hline 2 & 2 & 5 & 15 \\
3 & 5 & 16 & 64 \\
4 & 16 & 65 & 325 \\
5 & 65 & 326 & 1956 \\
6 & 326 & 1957 & 13699 \\
\hline
\end{tabular}

$$
\mathcal{P}\left(\delta_{s k 2}^{Q}\right)\left(\sum_{i \in Q} \xi_{i \varepsilon}+\mu_{s k 2}\right)=\mathcal{P}\left(\delta_{s k 1}^{Q}\right) \mu_{s k 1}+\mathcal{P}\left(\delta_{s k 2}^{Q-\{l\}}\right) \xi_{l s} \quad \text { for } k \notin Q, l=\operatorname{last}(Q)
$$

$$
\begin{gathered}
\mathcal{P}\left(\delta_{s k 2}^{Q}\right)\left(\sum_{i \notin Q} \xi_{i s}+\mu_{s k 2}\right)=\mathcal{P}\left(\delta_{s k 2}^{Q-\{l\}}\right) \xi_{l s} \quad \text { for } k \in Q, l=\operatorname{last}(Q) \\
\mathcal{P}\left(\delta_{s k 2}^{\bullet}\right)\left(\sum_{i=1}^{n}\left(\xi_{i s}+\mu_{s k 2}\right)=\mathcal{P}\left(\delta_{s k 1}^{\bullet}\right) \mu_{s k 1}\right. \\
\mathcal{P}\left(\delta_{s 0}^{\bullet}\right) \sum_{i=1}^{n} \xi_{i s}=\sum_{i=1}^{n} \mathcal{P}\left(\delta_{s+2}^{\bullet}\right) \mu_{s t 2}
\end{gathered}
$$

Equations (4.12, (4.13) apply to a state $\delta_{s k 1}^{Q} \in G_{s k 1}$ for the cases where the quelue is nonempty and empty respectively. Equations (4.14)-(4.16) apply to a state $\delta_{s k 2}^{Q} \in\left(i_{s k 2}\right.$ ats follows: (4.14) in the case with nonempty queue containing other clients but not $T_{k},(4.15)$ in the case with nonempty queue containing also $T_{k}$, and (4.16) in the case with enipty gurerue. The last equation (4.17) applies to the idle state $\delta_{s 0}^{\bullet} \in \mathcal{G}_{s 0}$.

The transition rate matrix of the continuous time MC for the simple client/server SRVN with $\mathbf{n}$ clients has the following form:

$$
\mathbf{Q}(n)=\left|\begin{array}{ccccc}
\mathbf{G}_{00} & \mathbf{H}_{01} & \mathbf{H}_{02} & \cdots & \mathbf{H}_{0 n} \\
\mathbf{H}_{10} & \mathbf{G}_{11} & \mathbf{H}_{12} & \cdots & \mathbf{H}_{1 n} \\
\mathbf{H}_{20} & \mathbf{H}_{21} & \mathbf{G}_{22} & \cdots & \mathbf{H}_{2 n} \\
\vdots & \vdots & \vdots & \ddots & \vdots \\
\mathbf{H}_{n 0} & \mathbf{H}_{n 1} & \mathbf{H}_{22} & \cdots & \mathbf{G}_{n n}
\end{array}\right|
$$

where: 
$\mathbf{G}_{\mathrm{ii}}$ is a diagonal submatrix whose nondiagonal elements represent transition rates between states inside group $\mathcal{G}_{a i}$, and whose diagonal elements are the sums (with negative sign) of all the rates for changing from the current state to any other state

$H_{i j}$ is a nondiagonal submatrix containing transition rates from states in $\mathcal{G}_{x i}$ to states in $\mathcal{G}_{s j}$.

The size of a diagonal submatrix $\mathrm{G}_{\mathrm{ij}}$ is $\left(L_{\mathrm{i}} \times L_{i}\right)$, and the size of a nondiagonal submatrix $\mathrm{H}_{\mathrm{ij}}$ is $\left(L_{i} \times L_{j}\right)$, where $L_{k}$ for $k=i, j$ is :

$$
L_{k}=\left\{\begin{array}{ll}
1 & \text { if } k=0 \\
N=N_{1}+N_{2} & \text { if } 1 \leq k \leq n
\end{array} \quad \text { for } k=i, j\right.
$$

where $N=\because+N_{2}$ is the number of states in group $\mathcal{G}_{s k}\left(N_{1}, N_{2}\right.$ are given by $(4.9),(4.10)$ respectively).

The size of $Q(n)$ is $(M \times M)$, where $M$ is given by (4.11).

A nondiagonal non-null element of a submatrix $G_{i i}$ has one of the following values: $\mu_{\text {oip }}$, $p=1,2$, if it corresponds to the end of phase $p$ of service for $T_{i}$, or $\xi_{k}, k=1, n$ if it corresponds to a request arrival from $T_{k}$. Submatrices $\mathbf{H}_{i j}$ are sparse, containing at most one non-null element per row. All non-null elements of a submatrix $\mathbf{H}_{\mathbf{i j}}$, for $i>0$ have the value $\mu_{s i 2}$, since they correspond to the end of phase 2 of service for task $T_{i}$.

\subsection{Task-directed aggregation of the $\mathrm{MC}$ for a simple client/server SRVN}

The arrival instant probabilities used for the queueing delay computation in an MVA-like algorithm for SRVN represent the probabilities of the following events:

- a RV request arriving from a client $T_{i}$ finds the server $T$, executing entry $k$, phase $p$

- a RV request from $T_{i}$ finds another client $T_{j}$ already in the queue.

These probabilities will be determined in section 4.4 from aggregated Markov Chain models of a client/server SRVN that show only the states of the following tasks:

a) the requesting client (whose arrival is studied) and the server 
b) the requesting client, one of its competitors, and the server.

The aggregation done by TDA-1 and TDA-2 (as defined in section 3.2.2) is described in the following subsections.

\subsubsection{MC aggregation for two designated tasks}

The "task-directed aggregation" of the MC for a simple client/server SRIN molld is discussed here, first for the simple case where the model has only two clients, and the'll for the. extended case with any number of clients. The set of designated tasks $D=\{i, s\}$ consid. ered in this subsection contains the client $T_{i}$ for which the arrival instant probabilities are. determined, and the server $T_{2}$.

By TDA-1 aggregation (as defined in section 3.2.2) all the states of the complet.e MC having some given combination of values $\sigma_{i} \sigma_{2}$ for the designated task states arc gromped together, disregarding the state of the other tasks. The new state vector indicates only the states of $T_{i}$ and $T_{s}$. The TDA-1 aggregation rule for the simple case with two clients is:

$$
\left(\sigma_{i} \sigma_{s}\right)=\bigcup_{\sigma, \in \mathcal{S}}\left(\sigma, \sigma_{3} \sigma_{s}\right)
$$

The information about the other client(s) being hidden by TDA-1 aggregation, we want also to raise the level of abstraction in the state set of $T_{n}$, such that it reflects only the relationship with the server. The queueing states showing the exact position of $T$ in quense are too detailed now, because they not only express the fact that $T_{i}$ is waiting for the server, but also the relative position with respect to other clients. Therefore, all queneing states of $T_{i}$ are aggregated by TDA-2 into a single state $q_{i s}$ :

$$
q_{i s}=\bigcup_{m=1, n} q_{i s}^{(m)}
$$

As a result, the state set of client $T_{i}$ becomes:

$$
\mathcal{S}_{i}^{\prime}=\left\{a_{i s}, q_{i s}, r_{i s}\right\}
$$

The state set $\mathcal{S}$, of the server given by (4.2) remains unchanged.

Figure 4.6 shows the exact aggregated MC obtained from the complete MC from Figur. 4.2, where the set of designated tasks is $D=\{i, s\}$. The aggregated MC is described liy the. following proposition. 


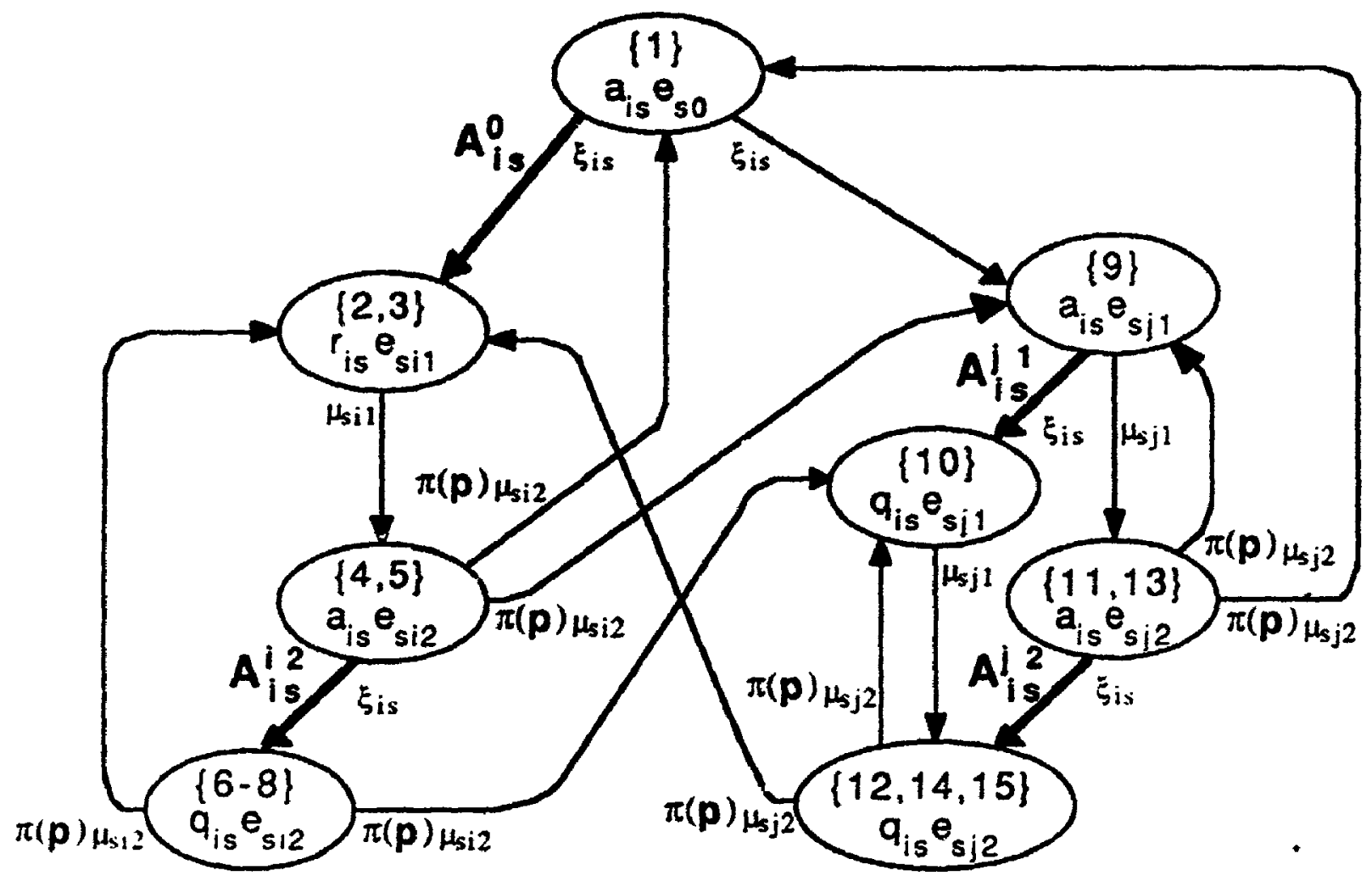

Figure 4.6: Aggregated MC of a simple client/server SRVN with two clients, for $D=\{i, s\}$ 
Proposition 1 The aggregated MC for $D=\{i, s\}$ obtained from the complete Markov chain model for a client/server SRVN with $n=2$ clients has three groups of aggregated stutes $G_{s k}^{\prime}$ for $k=0,2$, with the following composition:

$$
\mathcal{G}_{s k}^{\prime}= \begin{cases}\left\{\left(a_{i,} e_{s 0}\right)\right\} & \text { if } k=0 \\ \left\{\left(r_{i,} e_{s i 1}\right),\left(a_{i s} e_{s i 2}\right),\left(q_{i s} e_{s i 2}\right)\right\} & \text { if } k=i \\ \left\{\left(a_{i s} e_{s j 1}\right),\left(q_{i s} e_{s j 1}\right),\left(a_{i s} e_{s j 2}\right),\left(q_{i s} e_{s j 2}\right)\right\} & \text { if } k \neq i\end{cases}
$$

The size $L_{k}^{\prime}$ of a group $\mathcal{G}_{s k}^{\prime}$ is given by:

$$
L_{k}^{\prime}= \begin{cases}1 & \text { if } k=0 \\ 3 & \text { if } k=i \\ 4 & \text { if } k \neq i\end{cases}
$$

The transition rate matrix of the aggregated MC has the following form:

$$
\mathbf{Q}^{\prime}(2)=\left|\begin{array}{lll}
\mathbf{G}_{00}^{\prime} & \mathbf{H}_{01}^{\prime} & \mathbf{H}_{02}^{\prime} \\
\mathbf{H}_{10}^{\prime} & \mathbf{G}_{11}^{\prime} & \mathbf{H}_{12}^{\prime} \\
\mathbf{H}_{20}^{\prime} & \mathbf{H}_{21}^{\prime} & \mathbf{G}_{22}^{\prime}
\end{array}\right|
$$

where a submatrix $\mathbf{G}_{k \mathbf{k}}^{\prime}$ (for $k>0$ ) of size $\left(L_{k}^{\prime} \times L_{k}^{\prime}\right)$ contains aggregated transition rales between states inside group $\mathcal{G}_{s k}^{\prime}$, and a submatrix $\mathbf{H}_{\mathrm{km}}^{\prime}$ of size $\left(L_{k}^{\prime} \times L_{m}^{\prime}\right)$ contains aggrcgated transition rates from states in $\mathcal{G}_{s k}^{\prime}$ to states in $\mathcal{G}_{s m}^{\prime}$. The diagonal elements of $\mathbf{G}_{k k k}$ are the sums (with negative sign) of all the rates for changing from the current state to any oller state (including $\mathbf{G}_{00}^{\prime}$ that has a single element).

The size of matrix $Q^{\prime}(2)$ is $\left(M^{\prime} \times M^{\prime}\right)$, where $M^{\prime}=\sum_{k=0}^{n} L_{k}^{\prime}$.

The form of submatrices $\mathbf{G}^{\prime}$ and $\mathbf{H}^{\prime}$ is different for designated and nondesignated clients (assume $i \in D$ and $j \notin D$ ):

$$
\mathbf{G}_{\mathrm{ii}}=\left|\begin{array}{ccc}
-\mu_{i j 1} & \mu_{s i 1} & 0 \\
0 & -\xi_{i,-\mu s i 2} & \xi_{i s} \\
\pi_{31} \mu_{s i 2} & 0 & -\mu_{s i 2}
\end{array}\right| \quad G_{j i}^{\prime}=\left|\begin{array}{cccc}
-\xi_{i s}-\mu_{s j 1} & \xi_{i s} & \mu_{s j 1} & 0 \\
0 & -\mu_{s j 1} & 0 & \mu_{s j 1} \\
\pi_{31} \mu_{s j 2} & 0 & -\xi_{i s}-\mu_{s j 2} & \xi_{i s} \\
0 & \pi_{42} \mu_{s j 2} & 0 & -\mu_{s j 2}
\end{array}\right|
$$

Submatrices $\mathrm{H}_{\mathrm{ij}}^{\prime}$ are sparse, having at most one nonnull element per row :

$$
\begin{aligned}
& \mathbf{H}_{\mathbf{i j}}^{\prime}=\left|\begin{array}{cccc}
0 & 0 & 0 & 0 \\
\pi_{21} \mu_{, i 2} & 0 & 0 & 0 \\
0 & \pi_{32} \mu_{, i 2} & 0 & 0
\end{array}\right| \quad \mathbf{H}_{j i}^{\prime}=\left|\begin{array}{ccc}
0 & 0 & 0 \\
0 & 0 & 0 \\
0 & 0 & 0 \\
\pi_{41} \mu_{, j 2} & 0 & 0
\end{array}\right| \quad \text { for } i, j>0 \\
& \mathbf{H}_{\mathbf{i} 0}^{\prime}=\left|\begin{array}{c}
0 \\
\pi_{21} \mu_{s i 2} \\
0
\end{array}\right| \quad \mathbf{H}_{j 0}^{\prime}=\left|\begin{array}{c}
0 \\
0 \\
\pi_{31} \mu_{s i 2} \\
0
\end{array}\right| \quad \mathbf{H}_{\mathbf{0 i}}^{\prime}=\left|\begin{array}{llll}
\xi_{i s} & 0 & 0
\end{array}\right| \quad \mathbf{H}_{\mathbf{0 j}}^{\prime}=\left|\begin{array}{llll}
\xi_{j,} & 0 & 0 & 0
\end{array}\right|
\end{aligned}
$$


The factors $\pi$ on each row $r$ of $\mathbf{Q}^{\prime}(2)$, along all corresponding $\mathrm{G}^{\prime}$ and $\mathbf{H}^{\prime}$ submatrices, represent probabilities to choose the next destination group, and thus their sum equals one:

$$
\sum_{k=0}^{n} \sum_{h=1}^{L_{k}^{\prime}} \pi_{g h}=1 \text { where } g=r-\sum_{u=0}^{k-1} L_{u}^{\prime} \quad \text { for } r=1, M^{\prime}
$$

Proof. The aggregated space state is obtained directly by applying the aggregation rule (4.19) to the non-aggregated MC from Figure 4.2. The aggregated transition rates are obtained by applying Lemma 1 (section 3.2.2), which shows that the sum of aggregated transition rates from a given aggregated state due to a change of an active task state is independent of the non-aggregated $\mathrm{MC}$ probability vector.

Let us take as an example the aggregated state $\left(a_{i s} e_{s j 2}\right)$, that has outgoing transitions due to a change of either $a_{i s}$ or $e_{s j 2}$. By Lemma 1, these transitions have the following aggregated rates:

- transition from $\left(a_{i s} e_{s j 2}\right)$ to $\left(q_{i s} e_{s j 2}\right)$ with rate $\xi_{i s}$

- transitions from $\left(a_{i s} e_{s j 2}\right)$ to $\left(a_{i s} e_{s 0}\right)$ and to $\left(a_{i s} e_{s j 1}\right)$ with total rate $\mu_{s j 2}$.

All the other aggregated rates are obtained in a similar way.

The relation (4.26) is also obtained by applying Lemma 1 to every aggregated state in which the server is executing a second phase. Let $r$ be the row of the matrix $\mathbf{Q}^{\prime}(2)$ corresponding to such a state, and let $\mathcal{G}_{s l}^{\prime}$ be the group containing it. Thus, row $r$ lies along the following submatrices:

$$
\mathbf{G}_{1 \mathbf{k}}^{\prime} \text { for } k=l \text {, and } \mathbf{H}_{\mathbf{l k}}^{\prime} \text { for all } k \neq l
$$

The local row number $g$ corresponding to the global row number $r$ in any of the above submatrices is:

$$
g=r-\sum_{u=0}^{k-1} L_{u}^{\prime}
$$

By Lemma 1, the sum of all aggregated transition rates due to the end of the second phase is:

$$
\sum_{k=0}^{n} \sum_{h=1}^{L_{k}^{\prime}} \pi_{g h} \mu_{s j 2}=\mu_{s j 2}
$$


Relation 4.26 results immediately. It results also that the factors $\pi$ situated on row $r$ represent actual probabilities to choose between different possible transitions, and they depend on the probability vector of the non-aggregated MC.

The values of the diagonal elements on submatrices $G_{i j}^{\prime}$ which equals the sums (with negative sign) of all the rates for changing from ohe current state to any other state are simply obtained by adding the corresponding rates and by using relation 4.26 .

The derivation of the arrival instant probabilities equations from section 4.4 below uses the independence property of the sums of aggregated rates, but avoids the computation of choice probabilities $\pi$ that depend on the probability vector of the non-aggregated MC. 'Thus, the transition rates appearing in nondiagonal submatrices $\mathbf{H}^{\prime}$ are not used for the derivation of arrival instant probabilities equations. The nondiagonal submatrices have been given only for completeness.

\section{Aggregation of the MC with $n>2$ clients for $D=\{i, s\}$}

The exact aggregated version of the MC for $n$ clients contains $n+1$ groups of states $\mathcal{G}_{s k}^{\prime}$ for $k=0, n$ that have the same structure as the groups in the aggregated MC for two clients, described by Proposition 1 .

As in the case for two clients, the aggregation by TDA-2 given in (4.20) is applied to the queueing states of $T_{i}$. The aggregation by TDA-1 of the complete MC given by the balance equations (4.12)-(4.17) is conducted in such a way that all the states having a feasible combination of values $\sigma_{i}, \sigma_{s}$ for the designated task states are grouped together, disregarding the other tasks. An example of such an aggregation, where $D=\{2,4\}$, is given in Figure 4.7, that shows how are lumped together the states of the group $G_{41}$ from Figure 4.5 that is a part of the $\mathrm{MC}$ for a simple client/server SRVN model with $n=3$ clients.

Figure 4.8 illustrates the structure of the aggregated MC for $n>2$ clients, where the designated tasks are $D=\{i, s\}$. The similarity with the aggregated MC for two clients is given by the following theorem.

Theorem 1 The aggregated $M C$ obtained for $D=\{i, s\}$ from the $M C$ of a client/server $S R V N$ with $n>2$ clients has $(n+1)$ groups of aggregated states $\mathcal{G}_{s k}^{\prime}$, for $k=0, n$. The size. 


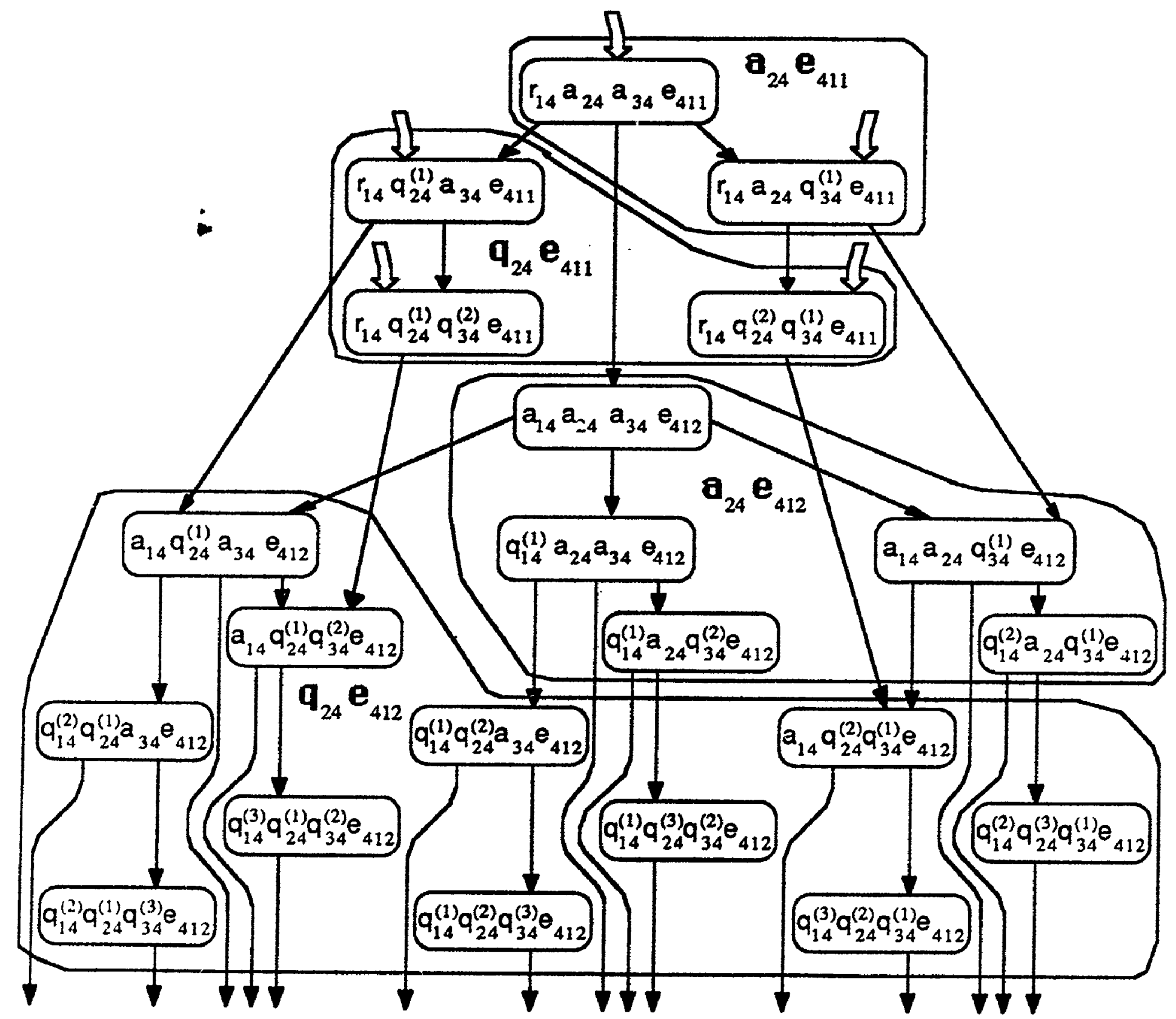

Figure 4.7: Aggregation for $D=\{2,4\}$ of group $G_{41}$ from Figure 4.5

95 


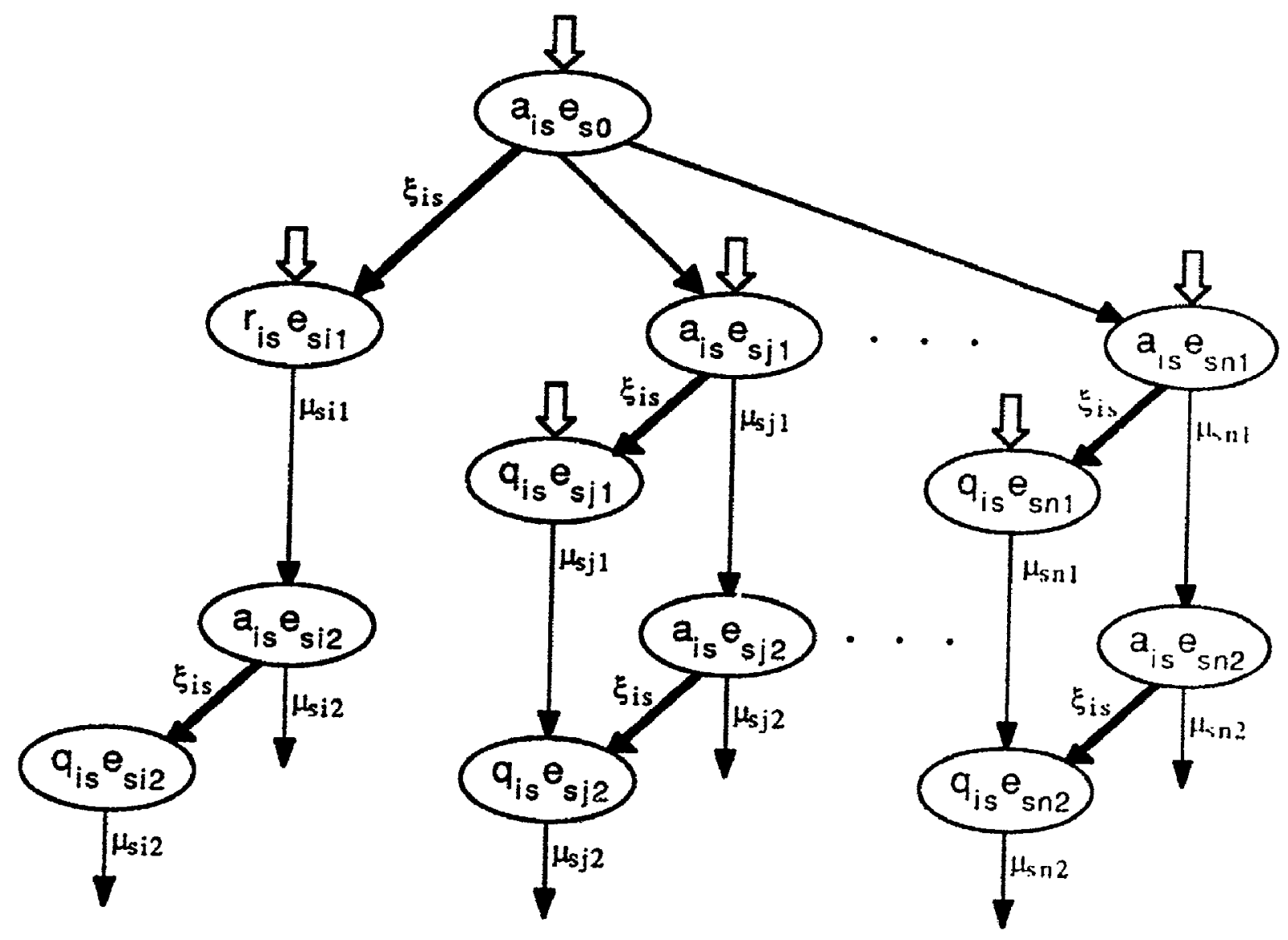

Figure 4.8: Aggregated MC of a simple client/server SRVN with $n$ clients, for $D=\{i, \diamond\}$ 
$L_{k}^{\prime}$ and composition of a group $\mathcal{G}_{s k}^{\prime}$ is the same as in the case for $n=2$, given by Proposition 1. The size $M^{\prime}$ of the aggregated $M C$ grows linearly with the number of clients n:

$$
M^{\prime}=\sum_{k=0}^{n} L_{k}^{\prime}
$$

The transition rate matrix of size $\left(M^{\prime} \times M^{\prime}\right)$ of the aggregated $M C$ has the following form:

$$
\mathbf{Q}^{\prime}(n)=\left|\begin{array}{ccccc}
\mathbf{G}_{00}^{\prime} & \mathbf{H}_{01}^{\prime} & \mathbf{H}_{02}^{\prime} & \cdots & \mathbf{H}_{0 \mathrm{n}}^{\prime} \\
\mathbf{H}_{10}^{\prime} & \mathbf{G}_{11}^{\prime} & \mathbf{H}_{12}^{\prime} & \cdots & \mathbf{H}_{1 \mathrm{n}}^{\prime} \\
\mathbf{H}_{20}^{\prime} & \mathbf{H}_{21}^{\prime} & \mathbf{G}_{22}^{\prime} & \cdots & \mathbf{H}_{2 \mathrm{n}}^{\prime} \\
\vdots & \vdots & \vdots & \ddots & \vdots \\
\mathbf{H}_{\mathrm{n} 0}^{\prime} & \mathbf{H}_{\mathrm{n} 1}^{\prime} & \mathbf{H}_{\mathrm{n2}}^{\prime} & \cdots & \mathbf{G}_{\mathrm{nn}}^{\prime}
\end{array}\right|
$$

where a submatrix $\mathrm{G}_{\mathrm{kk}}^{\prime}$ (for $\left.k>0\right)$ of size $\left(L_{k}^{\prime} \times L_{k}^{\prime}\right)$ contains aggregated transition rates between states inside group $\mathcal{G}_{s k}^{\prime}$, and a submatrix $\mathbf{H}_{\mathbf{k m}}^{\prime}$ of size $\left(L_{k}^{\prime} \times L_{m}^{\prime}\right)$ contains aggregated transition rates from states in $\mathcal{G}_{s k}^{\prime}$ to states in $\mathcal{G}_{s m}^{\prime}$. The diagonal elements of $\mathbf{G}_{k k}^{\prime}$ are the sums (with negative sign) of all the rates for changing from the current state to any other state (including $\mathbf{G}_{00}^{\prime}$ that has a single element).

The submatrices $\mathbf{G}^{\prime}$ and $\mathbf{H}^{\prime}$ have the same form as in Proposition 1, with the exception of a submatrix $\mathbf{H}_{\mathrm{jk}}^{\prime}$ where $k \notin D$ and $j \notin D$, that can appear only for $n>2$, and has the following form:

$$
\mathbf{H}_{\mathrm{jk}}^{\prime}=\left|\begin{array}{cccc}
0 & 0 & 0 & 0 \\
0 & 0 & 0 & 0 \\
\pi_{31} \mu_{s j 2} & 0 & 0 & 0 \\
0 & \pi_{42} \mu_{s j 2} & 0 & 0
\end{array}\right| \quad \text { for } j, k \neq i
$$

The factors $\pi$ on each row $r$ of $\mathbf{Q}^{\prime}(n)$, along all corresponding $\mathbf{G}^{\prime}$ and $\mathbf{H}^{\prime}$ submatrices, represent probabilities to choose the next destination group, and thus their sum equals one:

$$
\sum_{k=0}^{n} \sum_{h=1}^{L_{k}^{\prime}} \pi_{g h}=1 \quad \text { where } g=r-\sum_{u=0}^{k-1} L_{u}^{\prime} \quad \text { for } r=1, M^{\prime} \square
$$

Proof. By construction, the aggregated state vector of a client/server SRVN with $n>2$ clients shows only the designated task states $\left(\sigma_{i} \sigma_{s}\right)$, which take values from the same task states sets $\mathcal{S}_{i}^{\prime}(4.21)$ and $\mathcal{S}_{s}(4.2)$ as in the case with $n=2$ clients. The number of client states in $\mathcal{S}_{i}^{\prime}$ does not change with $n$, but the number of server states in $\mathcal{S}_{z}$ increases linearly with $n$. The aggregated state space, containing all feasible combinations from the product 
$\left(\mathcal{S}_{i}^{\prime} \times \mathcal{S}_{s}\right)$, is divided into $(n+1)$ groups $\mathcal{G}_{s k}^{\prime}$ of aggregated states (for $\left.k=0, n\right)$. The group $\mathcal{G}_{s 0}^{\prime}$, where $T_{s}$ is idle, contains all feasible combinations from the product $\left(\mathcal{S}_{i}^{\prime} \times\left\{\epsilon_{s o}\right\}\right)$, and a group $\mathcal{G}_{s k}^{\prime}$, for $k=1, n$, where $T_{s}$ is executing entry $k$, contains all feasible combinations from the product $\left(\mathcal{S}_{i}^{\prime} \times\left\{e_{s k 1}, e_{s k 2}\right\}\right)$. It results immediately that the composition of a group $\mathcal{G}_{s k}^{\prime}$ is exactly as in Proposition 1 , distinguishing three patterns: for server idle $(k=0)$, for designated task in service $(k=i)$, and for non-designated task in service $(k \neq i)$.

From the similarity of the groups of aggregated states it results the similarity of transition rate submatrices $\mathbf{G}^{\prime}$ and $\mathbf{H}^{\prime}$, that correspond to transitions inside and between groups.

The values for transition rates and the relation (4.31) are obtained as in Proposition I, by applying Lemma 1 to all aggregated states.

An important result of the previous theorem is (4.28), that shows that the size of the aggregated MC for $D=\{i, s\}$ grows linearly with the number of clients $n$, in spite of the fact that the size of the complete MC grows exponentially with $n$ as shown by (4.11).

\subsubsection{MC aggregation for three designated tasks}

In order to study the probabilities that an arriving request from $T_{i}$ finds ancther client $T_{j}$ in queue or in service, we aggregate the MC such that the set of designated tasks is $D=\{i, j, s\}$. For the simple case of a client/server SRVN with only two clients no aggregation is performed, since all the tasks are designated tasks. For the case with more than two clients, the MC is aggregated by TDA-1 and by TDA-2. An example of such an aggregation, where $D=\{1,2,4\}$, is given in Figure 4.9, that illustrates how are lumped together the states of the group $G_{41}$ from Figure 4.5, that is a part of the MC for a simple client/server SRVN model with $n=3$ clients.

Since only the relative position in queue of the designated clients is important here, thr. aggregated state set for $T_{i}$ is:

$$
\mathcal{S}_{i}^{\prime \prime}=\left\{a_{i s}, q_{i s}^{(a)}, q_{i s}^{(b)}, r_{i s}\right\}
$$

The aggregated state $q_{i s}^{(a)}$, obtained by TDA-2, means that $T_{i}$ is ahead of the other designat, $;$ client $T_{j}$, and $q_{i:}^{(b)}$ means that $T_{i}$ is behind $T_{j}$ :

$$
q_{i s}^{(a)}=\bigcup_{u=1}^{m-1} q_{i s}^{(u)} \quad q_{i s}^{(b)}=\bigcup_{u=m+1}^{n} q_{i s}^{(u)}
$$




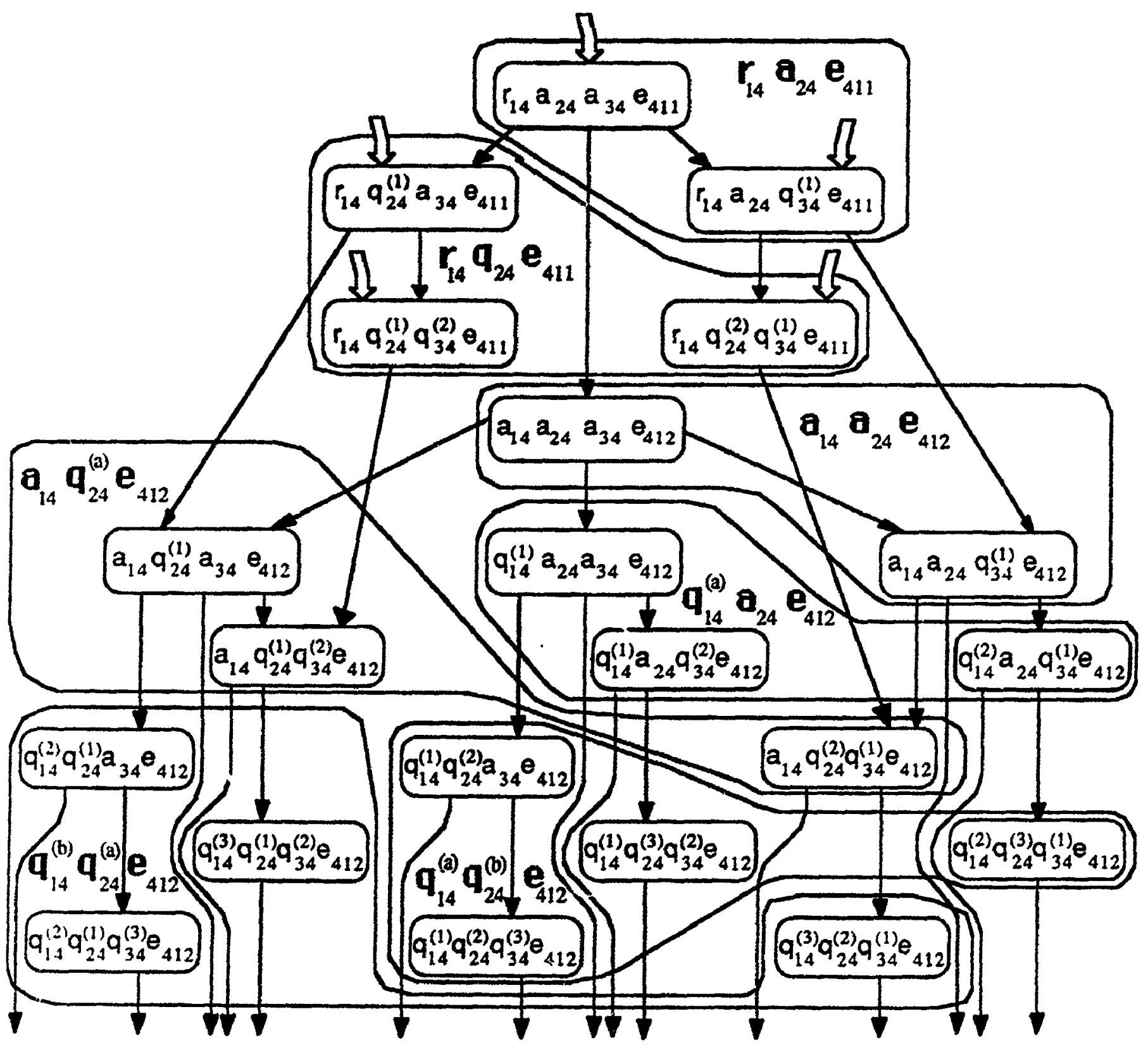

Figure 4.9: Aggregation for $D=\{1,2,4\}$ of group $G_{41}$ from Figure 4.5 
where $m$ is the exact position of $T_{j}$ in queue in a given state $\sigma$ (if $T$, is not in quenc. then $m=n$ ). The aggregated queueing states of $T_{j}$ are similarly described in function of the position of $T_{i}$. The state set for the server, given in (4.2), remains unchanged. The aggregated $\mathrm{MC}$ is described by the following theorem.

Theorem 2 The aggregated $M C$ for $D=\{i, j, s\}$ obtained from the complete Markou chain model for a client/server SRVN with $n>2$ clients has $(n+1)$ groups of aggregatrd stules $\mathcal{G}_{s k}^{\prime \prime}$ for $k=0, n$, with the following composition:

$$
\mathcal{G}_{a k}^{\prime \prime}= \begin{cases}\left\{\left(a_{i s} a_{j s} e_{s 0}\right)\right\} & \text { if } k=0 \\ \left\{\left(r_{i s} a_{j s} e_{s i 1}\right),\left(r_{i s} g\right.\right. \\ \left(a_{i s} a_{j s} e_{s i 2}\right),\left(q_{i s}^{(a)} a_{j s} e_{s i 1}\right), & \\ \left\{\left(a_{s i 2} a_{j s} e_{s k 1}\right),\left(q_{i s}^{(a)} q_{j s}^{(b)} e_{s i 2}\right),\left(a_{i s} q_{j s}^{(a)} e_{s i 2}\right),\left(q_{j s}^{(b)} e_{s k 1}^{(a)} e_{s i 2}\right)\right\},\left(q_{i s}^{(a)} q_{j s}^{(b)} e_{s k 1}\right),\left(a_{i s} q_{j s}^{(a)} e_{s k 1}\right),\left(q_{i s}^{(b)} q_{j s}^{(a)} e_{s k 1}\right) & \text { if } k=i \text { and } i \in l) \\ \left.\left(a_{i s} a_{j s} e_{s k 2}\right),\left(q_{i s}^{(a)} a_{j s} e_{s k 2}\right),\left(q_{i s}^{(a)} q_{j s}^{(b)} e_{s k 2}\right),\left(a_{i s} q_{j s}^{(a)} e_{s k 2}\right),\left(q_{i s}^{(b)} q_{j s}^{(a)} e_{s k 2}\right)\right\} & \text { if } k \notin D)\end{cases}
$$

The size $L_{k}^{\prime \prime}$ of a group $\mathcal{G}_{s k}^{\prime \prime}$ is given by:

$$
L_{k}^{\prime \prime}= \begin{cases}1 & \text { if } k=0 \\ 7 & \text { if } k \in D \\ 10 & \text { if } k \notin D\end{cases}
$$

The size $M^{\prime \prime}$ of the aggregated $M C$ grows linearly with the number of clients $n$ :

$$
M^{\prime \prime}=\sum_{k=0}^{n} L_{k}^{\prime \prime}
$$

The transition rate matrix of the aggregated $M C$ of size $\left(M^{\prime \prime} \times M^{\prime \prime}\right)$ has the following form:

$$
\mathbf{Q}^{\prime \prime}(n)=\left|\begin{array}{ccccc}
\mathbf{G}_{00}^{\prime \prime} & \mathbf{H}_{01}^{\prime \prime} & \mathbf{H}_{02}^{\prime \prime} & \cdots & \mathbf{H}_{0 \mathrm{n}}^{\prime \prime} \\
\mathbf{H}_{10}^{\prime \prime} & \mathbf{G}_{11}^{\prime \prime} & \mathbf{H}_{12}^{\prime \prime} & \cdots & \mathbf{H}_{1 \mathrm{n}}^{\prime \prime} \\
\mathbf{H}_{20}^{\prime \prime} & \mathbf{H}_{21}^{\prime \prime} & \mathbf{G}_{22}^{\prime \prime} & \cdots & \mathbf{H}_{2 \mathrm{n}}^{\prime \prime} \\
\vdots & \vdots & \vdots & \ddots & \vdots \\
\mathbf{H}_{\mathrm{n} 0}^{\prime \prime} & \mathbf{H}_{\mathrm{n} 1}^{\prime \prime} & \mathbf{H}_{\mathrm{n} 2}^{\prime \prime} & \cdots & \mathbf{G}_{\mathrm{nn}}^{\prime \prime}
\end{array}\right|
$$

where a submatrix $\mathrm{G}_{k k}^{\prime \prime}($ for $k>0)$ of size $\left(L_{k}^{\prime \prime} \times L_{k}^{\prime \prime}\right)$ contains aggregated transition ratrs between states inside group $\mathcal{G}_{s k}^{\prime \prime}$, and a submatrix $\mathbf{H}_{\mathbf{k m}}^{\prime \prime}$ of size $\left(L_{k}^{\prime \prime} \times L_{m}^{\prime \prime}\right)$ contains aljgrygald transition rates from states in $\mathcal{G}_{\text {sk }}^{\prime \prime}$ to states in $\mathcal{G}_{\text {sm }}^{\prime \prime}$. The diagonal elements of $\mathrm{G}_{\mathrm{kk}}^{\prime \prime}$ are the sums (with negative sign) of all the rutes for changing from the current state to any othr. $r$ state (including $\mathbf{G}_{00}^{\prime \prime}$ that has a single element). 
A diagonal submatrix $\mathbf{G}_{\mathrm{ii}}^{\prime \prime}$, where $i \in D$, is similar to a submatrix $\mathbf{G}_{\mathrm{in}}$ (4.4) from the transition rate matrix $\mathrm{Q} 2$ (4.3) of the non-aggregated $\mathrm{MC}$ with two clients :

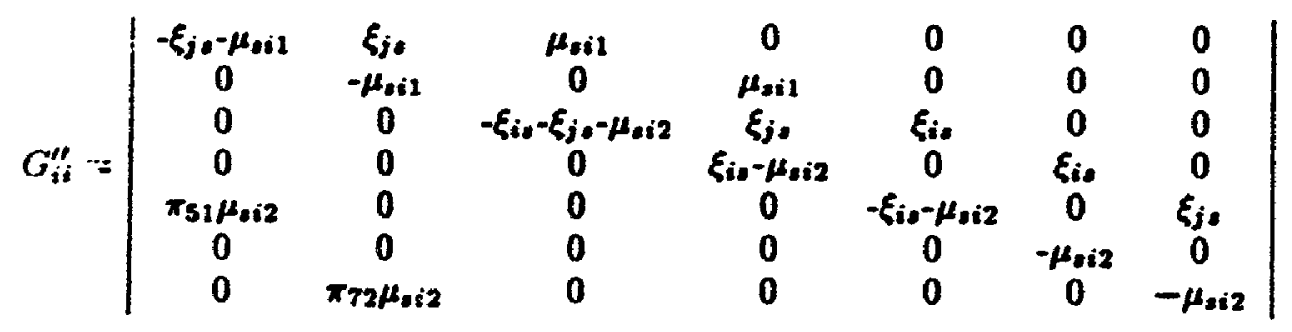

A diagonal submatrix $\mathrm{G}_{\mathrm{kk}}^{\prime \prime}$ has the following form for $k \notin D$ :

\begin{tabular}{|c|c|c|c|c|c|c|c|c|c|}
\hline$-\tilde{\xi}_{t,}-\xi_{j,-\mu_{2 k 1}}$ & $\xi_{j:}$ & $\xi_{i:}$ & 0 & 0 & $\mu_{3,1}$ & $\boldsymbol{0}$ & 0 & 0 & 0 \\
\hline 0 & $-\xi_{i, j}-\mu_{s k 1}$ & $\mathbf{0}$ & $\xi_{i \star}$ & 0 & 0 & $\mu_{s k 1}$ & 0 & 0 & 0 \\
\hline 0 & 0 & $-\xi_{j,}-\mu_{s k 1}$ & 0 & $\xi_{j,}$ & 0 & 0 & $\mu_{2 k 1}$ & 0 & 0 \\
\hline 0 & 0 & 0 & $-\mu_{8 k 1}$ & 0 & 0 & 0 & 0 & $\mu_{2 k 1}$ & 0 \\
\hline 0 & 0 & 0 & 0 & $-\mu_{s k 1}$ & 0 & 0 & 0 & 0 & $\mu_{2 k 1}$ \\
\hline$\pi_{61} \mu_{* k 2}$ & 0 & 0 & 0 & 0 & $-\xi_{i 0}-\xi_{j,}-\mu_{i k 2}$ & $\xi_{j=}$ & $\xi_{i o}$ & 0 & 0 \\
\hline 0 & $\pi_{72} \mu_{2 k 2}$ & 0 & 0 & 0 & 0 & $-\xi_{i t}-\mu_{k+2}$ & 0 & $\xi_{i s}$ & 0 \\
\hline 0 & 0 & $\pi_{83} \mu_{8 k 2}$ & 0 & 0 & 0 & 0 & $-\xi_{j a}-\mu_{s k 2}$ & 0 & $\xi_{j,}$ \\
\hline 0 & 0 & 0 & $\pi_{94} \mu_{d k 2}$ & 0 & 0 & 0 & 0 & $-\mu_{s k 2}$ & 0 \\
\hline 0 & 0 & 0 & 0 & $x_{10,5 \mu_{a k 2}}$ & 0 & $\mathbf{0}$ & 0 & 0 & $\begin{array}{l}-\mu_{x k 2} \\
4.377\end{array}$ \\
\hline
\end{tabular}

All submatrices $\mathrm{H}_{\mathrm{v}}^{\prime \prime}$ are sparse, containing at most one non-null element per row, and are given for completeness in appendix $A$.

The factors $\pi$ on each row $r$ of $\mathbf{Q}^{\prime \prime}(n)$, along all corresponding $\mathrm{G}^{\prime \prime}$ and $\mathbf{H}^{\prime \prime}$ submatrices, represent probabilities to choose the next destination group, and thus their sum equals one:

$$
\sum_{k=0}^{n} \sum_{h=1}^{L_{k}^{\prime \prime}} \pi_{g h}=1 \text { where } g=r-\sum_{u=0}^{k-1} L_{u}^{\prime \prime} \quad \text { for } r=1, M^{\prime \prime} \square
$$

Proof. By construction, the aggregated state vector of a client/server SRVN with $\boldsymbol{n}>\mathbf{2}$ clients shows only the designated task states $\left(\sigma_{i} \sigma_{j} \sigma_{s}\right)$, where $\sigma_{i}$ and $\sigma_{j}$ take values from the

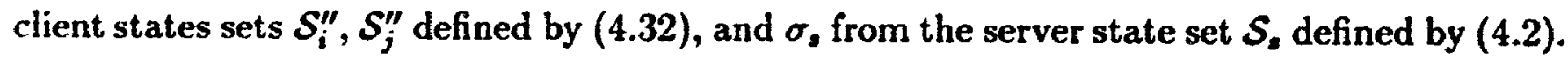
The number of clients states does not change with $n$, but the number of server states in $\mathcal{S}$, increases linearly with $\pi$. The aggregated state space, containing all feasible combinations from the product $\left(\mathcal{S}_{i}^{\prime \prime} \times \mathcal{S}_{j}^{\prime \prime} \times \mathcal{S}_{*}\right)$, is divided into $(n+1)$ groups $\mathcal{G}_{s k}^{\prime \prime}$ of aggregated states (for $\left.h^{\prime}=\mathbf{0}, \ldots, n\right)$.

The group $\mathcal{G}_{s 0}^{\prime \prime}$, where $T$, is idle, contains all feasible combinations from the product $\left(\mathcal{S}_{s}^{\prime \prime} \times \mathcal{S}_{j}^{\prime \prime} \times\left\{e_{s 0}\right\}\right)$. 
A group $\mathcal{G}_{a_{k}}^{\prime}$, for $k=1, n$, where $T$, is executing entry $k$, contains all feasible combinations from the product $\left(\mathcal{S}_{i}^{\prime \prime} \times \mathcal{S}_{j}^{\prime \prime} \times\left\{e_{s k 1}, \epsilon_{s k 2}\right\}\right)$.

From this it results immediately that the composition of a groap $\mathcal{G}_{s k}^{\prime \prime}$ is given by (1.3:3). We distinguish three patterns: for server idle $(k=0)$, for designated task in service $(k \in l)$ ). and for non-designated task in service $(k \notin D)$. It is easy to see that the groups $\mathcal{G}_{s i}^{\prime \prime}$ and $\mathcal{G}_{s j}^{\prime \prime}$, where the designated clients are in service, contain states with the same vectors $\sigma$ isi the groups $\mathcal{G}_{s i}$ and $G_{s j}$, respectively, from the non-aggregated MC of simple SRLN mudvI with two clients only (Figure 4.2). From the similarity of the groups of states it results the similarity of transition rate submatrices corresponding to transitions inside the groups: $\mathrm{C}_{\mathrm{ii}}^{\prime \prime}$ and $G_{j j}^{\prime \prime}$ (described by 4.36 , are similar to $G_{i j}$ and $G_{j j}$ (described by 4.4 ), respectively: The. only non-identical rates in 4.4 and 4.36 are the off-diagonal elements corresponding to everits.s. "end of second phase", affected by the choice probabilities $\pi$ (that are all 1 for the casce with 2 clients). The values for transition rates and the relation (4.38) are obtained as in Theormm 1 and Proposition 1, by applying Lemma 1 to the aggregated states giving the independeruce property of the sum of aggregated rates .

An important result of Theorem 2 is relation (4.34) showing that the size of the aggregated MC for $D=\{i, j, s\}$ grows linearly with the number of clients $n$, in spite of the fact that the size of the complete MC grows exponentially with $n$ as shown by (4.11).

\subsection{Derivation of equations for arrival instant proba- bilities}

The arrival instant probabilities equations for a client/server model with $n$ clients and sm. server represented in Figure 4.1. are derived in this section from the balance equations of a set of aggregated MCs obtained by task-directed aggregation, as described in section 4.3. Due to the fact that the transition rate submatrices of the aggregated MC's used for derivation have the same form for any number of clients $n$ (as proven by Theorems 1 and 2) in spite of the strong dependency on $n$ of the non-aggregated MCs (as shown in sertion 4.2), the arrival instant probabilities equations will have the same form for any $n$. However, their number grows with $n^{3}$, as will be shown in section 4.5 . 
The exact MVA equations (3.1)-(3.5) for phase durations and throughputs for an SRVN model presented in section 3.1.1 take a simplified form in this case, being condensed to the following equation:

$$
F_{i s}=\frac{1}{\xi_{i s}^{-1}+w_{i s}+x_{s i 1}} \quad \text { for } i=1, n
$$

where:

$F_{i s}=$ throughput of $\mathrm{RV}$ requests from $T_{i}$ to $T_{\text {. }}$

$w_{i,}=$ mean queueing delay for a $\mathrm{RV}$ request from $T_{i}$ to $T_{2}$ (without service)

$\xi_{i,}=$ rate of the exponentially distributed period spent by $T_{i}$ in state $a_{i s}$

$x_{s i 1}=$ service time of $T_{v}$, phase 1 , when serving $T_{i}$ (exponentially distributed with rate $\mu_{s i 1}$ ).

The queueing delay $w_{i}$ suffered by a request from $T_{i}$ at the server $T_{3}$ is computed as:

$$
w_{i s}=\sum_{j=1}^{n}\left[A_{i s}^{j 1}\left(x_{s j 1}+x_{s j 2}\right)+A_{i s}^{j 2} x_{s j 2}+B_{i s j}\left(x_{s j 1}+x_{s j 2}\right)\right]
$$

where $A$ and $B$ are the arrival instant probabilities defined as follows:

$A_{i,}^{J p}=$ the probability that a $\mathrm{RV}$ request from $T_{i}$ arriving to $T$, finds the server busy in phase $p$ serving a request from another client $T_{j}$

$B_{i s,}=$ the probability that a RV request from $T_{i}$ arriving to $T$, finds $T_{j}$ already in the queue (not in service).

Another arrival instant probability also used in the derivation process is:

$C_{i s}=$ the probability that a $\mathrm{RV}$ request from $T_{i}$ arriving to $T_{0}$ does not see $T_{j}$ either in queue or in service.

Since the arrival of RV requests is studied in function of the state of the server, it is useful to define the following components of $B$ and $C$ :

$B_{i s j}^{k p}=$ the probability that a $\mathrm{RV}$ request from $T_{i}$ arriving when $T$, is executing entry $k$, phase $p$, finds $T_{j}$ in the queue

$C_{i s,}^{k p}=$ the probability that a RV request from $T_{i}$ arriving when $T$, is executing entry $k$, phase $p$, does not see $T_{j}$ either in queue or in service. 


\section{Arrival instant probabilities equations}

For a global view, we list first the set of simultaneous equations for the arrival instant. probabilities, continuing with their derivation in the rest of the section.

$$
\begin{gathered}
A_{i s}^{i 2}=\frac{\xi_{i s}}{\xi_{i s}+\mu_{s i 2}} \quad \text { for } i=1, n \\
A_{i s}^{j 2}=\frac{\mu_{s j 1}}{\xi_{i s}+\mu_{s j 2}} A_{i s}^{j 1} \quad \text { for } i, j=1, n ; i \neq j \\
\left(\frac{\mu_{s j 1}}{\xi_{i s}}+1\right) A_{i s}^{j 1}+B_{i s j}=\frac{F_{j s}}{F_{i s}} \quad \text { for } i, j=1, n ; i \neq j \\
B_{i s j}=B_{i s j}^{i 2}+B_{i s j}^{j 2}+\sum_{k \neq i, j} \sum_{p}\left(A_{i s}^{k p}-C_{i s j}^{k p}\right) \quad \text { for } i, j, k=1, n ; i \neq j ; k \neq i, j \\
B_{i s j}^{i 2}=\frac{\xi_{i s}}{\xi_{i s}+\mu_{s i 2}} \cdot \frac{F_{j s}}{F_{i s}}\left[B_{j s i}+\left(1+\frac{\mu_{s i 1}}{\xi_{i s}+\xi_{j s}+\mu_{s i 2}}\right) A_{j s}^{i 1}\right] \quad \text { for } i, j=1, n ; i \neq j \\
B_{i s j}^{j 2}=\frac{\xi_{j s}}{\left(\xi_{i s}+\xi_{j s}+\mu_{s j 2}\right)} A_{i s}^{j 2} \quad \text { for } i, j=1, n ; i \neq j \\
C_{i s j}^{k 1}=\frac{F_{j s} \xi_{j s}^{-1}}{F_{k s} \mu_{s k 1}^{-1}} A_{i s}^{k 1} A_{j s}^{k 1} \quad \text { for } i, j, k=1, n ; i \neq j ; k \neq i, j \\
C_{i s j}^{k 2}=\frac{\mu_{s k 1}}{\xi_{i s}+\xi_{j s}+\mu_{s k 2}} C_{i s j}^{k 1} \quad \text { for } i, j=1, n ; i \neq j ; k \neq i, j
\end{gathered}
$$

Equations (4.41)-(4.46), (4.48) are exact, the only approximation being equation (4.17). Equations (4.47) and (4.48) are used only when there are more than two clients.

The equations for arrival instant probabilities are derived from the balance equations of a set of aggregated MCs: (4.41) and (4.42) from aggregated MCs showing only the statces of two tasks, and (4.43)-(4.46) from aggregated MCs showing the states of three tasks. 


\section{Arrival instant probabilities defined as conditional probabilities}

We begin by deriving some useful relationships. Let us select a client $T_{i}$, and determine the arrival instant probabilities from the $\mathrm{MC}$ in Figure 4.2, where $n=2$. First, the throughput $F_{i}$ of RV requests from $T_{i}$ equals the sum of transition flows corresponding to all "arrival from $T_{i}$ " events, as follows:

$$
F_{i 1}=\phi_{1,2}+\phi_{1,6}+\phi_{5,7}+\phi_{9,10}+\phi_{11,12}+\phi_{13,15}
$$

where $\phi_{g h}$ is the transition flow from state $g$ to state $h$ (computed as transition_rate $x$ state_probability).

The probability that an arrival from $T_{i}$ happens through a given transition is computed as the ratio of the transition flow over the request throughput: $\phi_{g h} / F_{i s}$. For example, the probabilities $A, B$ and $C$ for client $T_{i}$ in the case with two clients are determined as follows:

$$
\begin{gathered}
A_{i s}^{i 2}=\frac{\phi_{4,6}+\phi_{5,7}}{F_{i s}} \quad A_{i s}^{j 1}=\frac{\phi_{9,10}}{F_{i,}} \quad A_{i s}^{j 2}=\frac{\phi_{11,12}+\phi_{13,15}}{F_{i s}} \\
A_{i s}^{0}=\frac{\phi_{1,2}}{F_{i s}} \\
B_{i, j}=\frac{\phi_{5,7}+\phi_{13,15}}{F_{i s}} \\
C_{i s j}=\frac{\phi_{1,2}+\phi_{4,6}+\phi_{11,12}}{F_{i s}}
\end{gathered}
$$

The arrival instant probabilities can be also defined as conditional probabilities. Thus, $A_{i s}^{k p}$ and $A_{i}^{0}$ are defined as conditional probabilities of different server states conditioned on the fact that $T$, is in state $a_{i s}$ :

$$
A_{i s}^{k p}=P\left(e_{s k p} \mid a_{i s}\right)=\frac{P\left(e_{s k p} \cap a_{i s}\right)}{P\left(a_{i s}\right)} \quad \text { for } k=i, j
$$

The probability that $T_{i}$ finds the server in idle state is:

$$
A_{i s}^{0}=P\left(\varepsilon_{s 0} \mid a_{i s}\right)=\frac{P\left(a_{i s} \cap e_{s 0}\right)}{P\left(a_{i s}\right)}
$$

It is easy to show that definitions (4.54) and (4.55) are equivalent to (4.50) and (4.51), respectively. 
$B_{i n j}$ and $C_{i, j}$ can also be defined as conditional probabilities of different states of $T$; conditioned on the fact that $T_{i}$ is in state $a_{i s}$, obtaining relations equivalent to (4.52) and (4.53).

$$
\begin{aligned}
& B_{i, j}=P\left(a_{j s} \mid a_{i s}\right)=\frac{P\left(a_{i,} \cap q_{j s}\right)}{P\left(a_{i s}\right)} \quad \text { for } i, j=1, n ; i \neq j \\
& C_{i s j}=P\left(a_{j s} \mid a_{i s}\right)=\frac{P\left(a_{i s} \cap a_{j s}\right)}{P\left(a_{i s}\right)} \quad \text { for } i, j=1, n ; i \neq j
\end{aligned}
$$

The components of $B$ and $C$ probabilities are defined as conditional probabilities as follows:

$$
\begin{aligned}
& B_{i s j}^{k p p}=P\left(q_{j s}^{(a)} \cap e_{s k p} \mid a_{i s}\right)=\frac{P\left(a_{i s} \cap q_{j s}^{(a)} \cap e_{s k p}\right)}{P\left(a_{i s}\right)} \\
& C_{i s j}^{k p}=P\left(a_{j s} \cap e_{s k p} \mid a_{i s}\right)=\frac{P\left(a_{i s} \cap a_{j s} \cap e_{s k p}\right)}{P\left(a_{i s}\right)}
\end{aligned}
$$

Due to RV characteristics, when $T_{j}$ is in state $r_{j s}, T_{s}$ is serving $T_{j}$ in state $e_{s, 1}$. Therefore we have:

$$
P\left(r_{j s} \mid a_{i s}\right)=P\left(e_{s j 1} \mid a_{i s}\right)=A_{i s}^{j 1}
$$

Since the states $a_{j s}, q_{j s}$ and $r_{j s}$ of $T_{j}$ are mutually exclusive and exhaustive, we have the following relation:

$$
A_{i s}^{j 1}+B_{i s j}+C_{i s j}=1
$$

The same reasoning applied to $T$, states gives:

$$
\sum_{k=i, j} \sum_{p} A_{i s}^{k p}+A_{i s}^{0}=1
$$

\section{Derivation of equations (4.41), (4.42)}

The MC of Figure 4.8, aggregated for $D=\{i, s\}$, is used to derive equations (4.41) and (4.42). As a consequence of Theorem 1 showing that the size and composition of a grom, of aggregated states $\mathcal{G}_{s k}^{\prime}(k=1, n)$ is the same for any number of clicats $n$, the cquations derived are valid for any $n$. In fact, the balance equations used in the derivation process involve only state independent transition rates contained in the diagonal submatrices ( ${ }^{\prime}$ (4.23; of the transition rate matrix $Q^{\prime}$ (4.29).

The following notations are used for MC states:

$\mathcal{P}($ vector $)=$ probability of the state indicated by vector 
$\Phi($ vector $)=$ total input (output) flow of the indicated state.

It is known that a request from $T_{i}$ cannot arrive when $T_{s}$ is serving $T_{i}$ in first phase, thus $A_{i s}^{i 1}$ is always zero. However, a request from $T_{i}$ may see the server busy in phase 2 due to a previous request from $T_{i}$ itself with probability $A_{i s}^{i 2}$ (called also "overtaking" robability):

$$
A_{i s}^{i 2}=\frac{\mathcal{P}\left(a_{i s} e_{s i 2}\right)}{\xi_{i s}^{-1} F_{i s}}
$$

$\Lambda$ state probability can be expressed as the flow over the rate:

$$
\mathcal{P}\left(a_{i s} e_{s i 2}\right)=\frac{\Phi\left(a_{i s} e_{s i 2}\right)}{\mu_{s i 2}+\xi_{i s}}
$$

Due to the fact that $\left(r_{i s} e_{s i 1}\right)$ represents phase 1 of service for $T_{i}$ we have :

$$
\Phi\left(a_{i s} e_{s i 2}\right)=\Phi\left(r_{i s} e_{s i 1}\right)=F_{i s}
$$

From (4.63), (4.64) and (4.65) we get (4.41).

To obtain equation (4.42) we begin with definition (4.54), which gives:

$$
A_{i s}^{j 1}=\frac{\mathcal{P}\left(a_{i s} e_{s j 1}\right)}{\xi_{i s}^{-1} F_{i s}} \quad A_{i s}^{j 2}=\frac{\mathcal{P}\left(a_{i s} e_{s j 2}\right)}{\xi_{i s}^{-1} F_{i s}}
$$

From the balance equations of the aggregated MC in Figure 4.8 we get:

$$
\mathcal{P}\left(a_{i s} e_{s j 2}\right)=\frac{\mu_{s j 1} \mathcal{P}\left(a_{i s} e_{s j 1}\right)}{\xi_{i s}+\mu_{s j 2}}
$$

Equation (4.42) results from (4.66) and (4.67).

The utilizations of server phases in which the arrivals occur are $U_{s j 1}=F_{j s} \mu_{s j 1}^{-1}$ and $U_{s, 2}=F_{j s} \mu_{s j 2}^{-1}$ respectively. It results from the previous relation that the arrival probabilities $A$ are not proportional with the utilization of the server phases in which the arrivals occur:

$$
\frac{A_{i s}^{j 1}}{A_{i s}^{j 2}} \neq \frac{U_{s j 1}}{U_{s j 2}}
$$

\section{Derivation of equations (4.43) $-(\mathbf{4 . 4 8 )}$}

The arrival instant probability equations (4.43)-(4.48) will be derived from a $\mathrm{MC}$ showing only the states of the server $T_{s}$ and of two contenders $T_{i}$ and $T_{j}$. For the simple case with $n=2$ clients this is the complete MC of the client/server SRVN represented in Figure 4.2, 
while for $n>2$ this is an aggregation of the complete $\mathrm{MC}$, whose properties are given by Theorem 2. As a consequence of Theorem 2 showing that the size and composition of a group of aggregated states $\mathcal{G}_{s k}^{\prime \prime}(k=1, n)$ is the same for any number $n$ of clients, the arrival instant probabilities equations are valid for any $n$. In the derivation are used, in fact, only balance equations involving state independent transition rates, contained in the diagonal submatrices (given by (4.4) for $n=2$ and by (4.36) and (4.37) for $n>2$ ) of the transition rate matrix. The state dependent transition rates (affected by $\pi$ choice probabilities, as described by Theorem 2) are not used in derivation; the corresponding transition flows are expressed instead in function of the arrival probabilities and request throughputs (as shown in relation (4.68) below).

Equation (4.43) relating the arrival probabilities $A_{i s}^{j 1}$ and $B_{i s j}$ is obtained by analysing the group of states $\mathcal{G}_{s j}$ for $n=2$ or $\mathcal{G}_{s j}^{\prime \prime}$ for $n>2$, where task $T_{j}$ is in service. Since groupss $\mathcal{G}_{s j}$ and $\mathcal{G}_{s j}^{\prime \prime}$ have a similar structure, we use for illustration Figure 4.2, where the group $\mathcal{G}_{s j}$ contains the states 9 to 15 .

The presence of $T_{i}$ in queue at the moment when the service for task $T$, begins implies that the request from $T_{i}$ found $T_{j}$ already in queue when it arrived. In terms of MC states and transitions, this means that the input flow to state $\left(q_{i s}^{(a)} r_{j s} e_{s j 1}\right)$ from all other states except $\left(a_{i s} r_{j}, e_{s j 1}\right)$ is equal to the fraction of $T_{i}$ requests finding $T_{j}$ in the queue, i.e. $F_{i s} B_{i s j}$. (This flow is coming to $\left(q_{i s}^{(a)} r_{j s} e_{s j 1}\right)$ from states of the form $\left(q_{i s}^{(b)} q_{j s}^{(a)} e_{s k 2}\right)$, for all $\left.k=1, n\right)$. On the other hand, the input flow to state $\left(q_{i s}^{(a)} r_{j s} e_{s j 1}\right)$ from state $\left(a_{i s} r_{j s} e_{s j 1}\right)\left(\phi_{9,10}\right.$ in Figure 4.2) represents the fraction of $T_{i}$ requests arriving when $T_{i}$ is served in the first phase, i.e. $F_{i s} A_{i s}^{11}$. Thus, we can exptess the input flow to $\left(q_{i s}^{(a)} r_{j s} e_{s j 1}\right)$ as a function of arrival probabilities and throughput requests, avoiding the use of balance equations that imply, for $n>2$, state dependent transition rates (see Theorem 2).

$$
\Phi\left(q_{i s}^{(a)} r_{j s} e_{s j 1}\right)=F_{i s}\left(A_{i s}^{j 1}+B_{i s j}\right)
$$

We consider now the transition flows that correspond to the end of phase 1 of service for $T_{J}$ ( $\phi_{9,11}$ and $\phi_{10,12}$ in Figure 4.2), whose sum equals the throughput $F_{j s}$ of requests from $T$;

$$
\phi_{9,11}+\phi_{10,12}=F_{\jmath s}
$$


Transition flow $\phi_{9,11}$ can be written as:

$$
\phi_{9,11}=\mu_{s j 1} \mathcal{P}\left(a_{i s} r_{j s} e_{s j 1}\right)=\frac{\mu_{s j 1}}{\xi_{i s}} A_{i s}^{j 1} F_{i s}
$$

Transition flow $\phi_{10,12}$ equals the output flow from $\left(q_{i s}^{(a)} r_{j s} e_{s j 1}\right)$ :

$$
\phi_{10,12}=\Phi\left(\left(q_{i s} r_{j s} e_{s j 1}\right)\right.
$$

Equation (4.43) results immediately from (4.69), (4.70), (4.71) and (4.68).

Equation (4.44) is obtained by decomposing $B_{i s j}$ in function of the server states as follows:

$$
B_{i s j}=\sum_{k} \sum_{p} B_{i s j}^{k p}+B_{i s j}^{0}
$$

where a component is defined by (4.58). Three of the components, i.e. $B_{i s j}^{0}, B_{i s j}^{i 1}$, and $B_{i s j}^{j 1}$, are always zero for the following reasons:

- the queue is empty when the server is idle, thus an arrival from $T_{i}$ cannot see $T_{j}$ in queue

- a request from $T_{i}$ cannot arrive when $T_{i}$ is "in $\mathrm{RV}^{\text {" with the server }}$

- when $T_{j}$ is served in phase 1 , it cannot be also in queue to be seen by $T_{i}$.

Thus, relation 4.72 becomes

$$
B_{i s j}=B_{i s j}^{i 2}+B_{i s j}^{j 2}+\sum_{k \neq i, j} \sum_{p} B_{i s j}^{k p}
$$

From the definitions of $A_{i s}^{k p},(4.54), B_{i s j}^{k p}(4.58)$, and $C_{i s j}^{k p}(4.59)$ it results:

$$
A_{i s}^{k p p}=B_{i s j}^{k p}+C_{i s j}^{k p}
$$

Equation (4.44) results immediately from (4.73) and (4.74).

Equation (4.45) is derived from the group of states $\mathcal{G}_{s i}$ for $n=2$ (containing states 2-7 in Figure 4.2) or from $\mathcal{G}_{s i}^{\prime \prime}$ for $n>2$. By definition, $B_{i j j}^{i 2}$ is given by the following ratio of flows:

$$
B_{i s j}^{i 2}=\frac{\xi_{i s} \mathcal{P}\left(a_{i s} q_{j s}^{(a)} e_{s i 2}\right)}{F_{i s}}
$$


From the MC balance equations we get:

$$
\mathcal{P}\left(a_{i s} q_{j s}^{(a)} e_{s i 2}\right)=\frac{\xi_{j s} \mathcal{P}\left(a_{i s} a_{j s} e_{s i 2}\right)+\mu_{s i 1} \mathcal{P}\left(r_{i s} q_{j s}^{(a)} e_{s i 1}\right)}{\xi_{i s}+\mu_{s i 2}}
$$

The first state probability on the right side of 4.76 is used in the definition of probability $C_{i s j}^{i 2}$ as follows:

$$
C_{i s j}^{i 2}=\frac{\xi_{i s} \mathcal{P}\left(a_{i s} a_{j s} e_{s i 2}\right)}{F_{i s}}
$$

The second state probability on the right side of 4.76 is obtained from a relation similar to (4.68) as:

$$
\mathcal{P}\left(r_{i s} q_{j s}^{(a)} e_{s j 1}\right)=\frac{F_{j s}\left(A_{j s}^{i 1}+B_{j s i}\right)}{\mu_{s i 1}}
$$

By using the relations $(4.75)-(4.78)$, it results:

$$
B_{i s j}^{i 2}=\frac{\xi_{j s}}{\xi_{i s}+\beta_{i s i 2}} C_{i s j}^{i 2}+\frac{\xi_{i s}}{\xi_{i s}+\mu s i 2} \frac{F_{j s}}{F_{i s}}\left(A_{j s}^{i 1}+B_{j s i}\right)
$$

We determine now the probability $C_{i s j}^{i 2}$ by using (4.77). From the MC balance equations we have:

$$
\mathcal{P}\left(a_{i s} a_{j s} e_{s i 2}\right)=\frac{\mu_{s i 1}}{\xi_{i s}+\xi_{j s}+\mu_{s i 2}} \mathcal{P}\left(r_{i s} a_{j s} e_{s i 1}\right)
$$

From the definition of $A_{j s}^{i 1}$ as a ratio of flows we have:

$$
\mathcal{P}\left(r_{i s} a_{j s} e_{s i 1}\right)=\frac{A_{j s}^{i 1} F_{j s}}{\xi_{j s}}
$$

By replacing 4.81 into $4.80 C_{i s j}^{i 2}$ results as:

$$
C_{i s j}^{i 2}=\frac{\mu_{s i 1}}{\xi_{i s}+\xi_{j s}+\mu_{s i 2}} \cdot \frac{F_{j s} \xi_{j s}^{-1}}{F_{i s} \xi_{i s}^{-1}} A_{j s}^{i 1}
$$

Equation (4.45) results immediately from (4.79) and 4.82).

A similar, but much simpler derivation produces equation (4.46) for $B_{i g j}^{j 2}$ from the group of states $\mathcal{G}_{s j}$ for $n=2$ (containing states $9-15$ in Figure 4.2) or from $\mathcal{G}_{s i}^{\prime \prime}$ for $n>2$, where: task $T_{j}$ is in service. Similar to $(4.75), B_{i s j}^{j 2}$ is given by:

$$
B_{i s j}^{j 2}=\frac{\xi_{i s} \mathcal{P}\left(a_{i s} q_{j s}^{(\alpha)} e_{s j 2}\right)}{F_{i s}}
$$


From the MC balance equations we get:

$$
\mathcal{P}\left(a_{i,} q_{j s}^{(a)} e_{s j 2}\right)=\frac{\xi_{j s} \mathcal{P}\left(a_{i s} a_{j s} e_{s j 2}\right)}{\xi_{i s}+\mu_{s j 2}}
$$

The state probability on the right side of 4.84 is used in the definition of probability $C_{i * j}^{j 2}$ as follows:

$$
C_{i s j}^{j 2}=\frac{\xi_{i s} \mathcal{P}\left(a_{i s} a_{j s} e_{s j 2}\right)}{F_{i s}}
$$

By using the relations (4.83) and (4.85) we obtain:

$$
B_{i s j}^{j 2}=\frac{\xi_{j,}}{\xi_{i s}+\mu s j 2} C_{i s j}^{j 2}
$$

We determine now the probability $C_{i s j}^{j 2}$ by using (4.85). From the $\mathrm{MC}$ balance equations we have:

$$
\mathcal{P}\left(a_{i s} a_{j s} e_{s j 2}\right)=\frac{\mu_{s j 1}}{\xi_{i s}+\xi_{j s}+\mu_{s j 2}} \mathcal{P}\left(a_{i s} r_{j s} e_{s j 1}\right)
$$

Similar to (4.81) we can write:

$$
\mathcal{P}\left(a_{i s} r_{j *} e_{s j 1}\right)=\frac{A_{i s}^{j 1} F_{i s}}{\xi_{i s}}
$$

By replacing 4.88 into $4.87 C_{i s j}^{j 2}$ results as:

$$
C_{i s j}^{j 2}=\frac{\mu_{s j 1}}{\xi_{i s}+\xi_{j \psi}+\mu_{s j 2}} A_{i s}^{j 1}
$$

Equation (4.46) results from (4.79), (4.89) and (4.43).

The equations (4.47) and (4.48) for $C_{i \neq j}^{k 1}$ and $C_{i s j}^{k 2}$ are used only for $n>2$. Figure 4.10 shows the group of aggregated states $\mathcal{G}_{\jmath k}^{\prime \prime}$ where a non-designated task $T_{k}$ is in service.

$C_{i s j}^{k p}$ is the probability that an arrival from $T_{i}$ finds the server in phase $p$, entry $k$ and does not find $T_{j}$ at the server, being defined similarly to (4.77):

$$
C_{i, j}^{k p p}=\frac{\xi_{i,} \mathcal{P}\left(a_{i s} a_{j, s} e_{s k p}\right)}{F_{i s}}
$$

The approximation introduced in equation (4.47) is based on the assumption that the arrival to $T_{8}$ from $T_{i}$ is independent from the arrival to $T_{\text {s }}$ from $T_{j}$ for the period of time when $T_{3}$

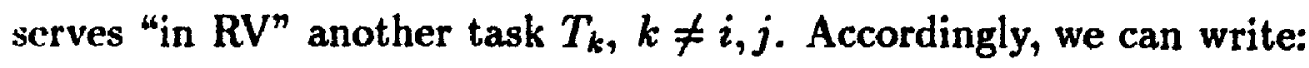

$$
P\left(a_{i s} \mid e_{s k 1}\right)=P\left(a_{i s} \mid\left(a_{j s} \cap e_{s k 1}\right)\right)
$$




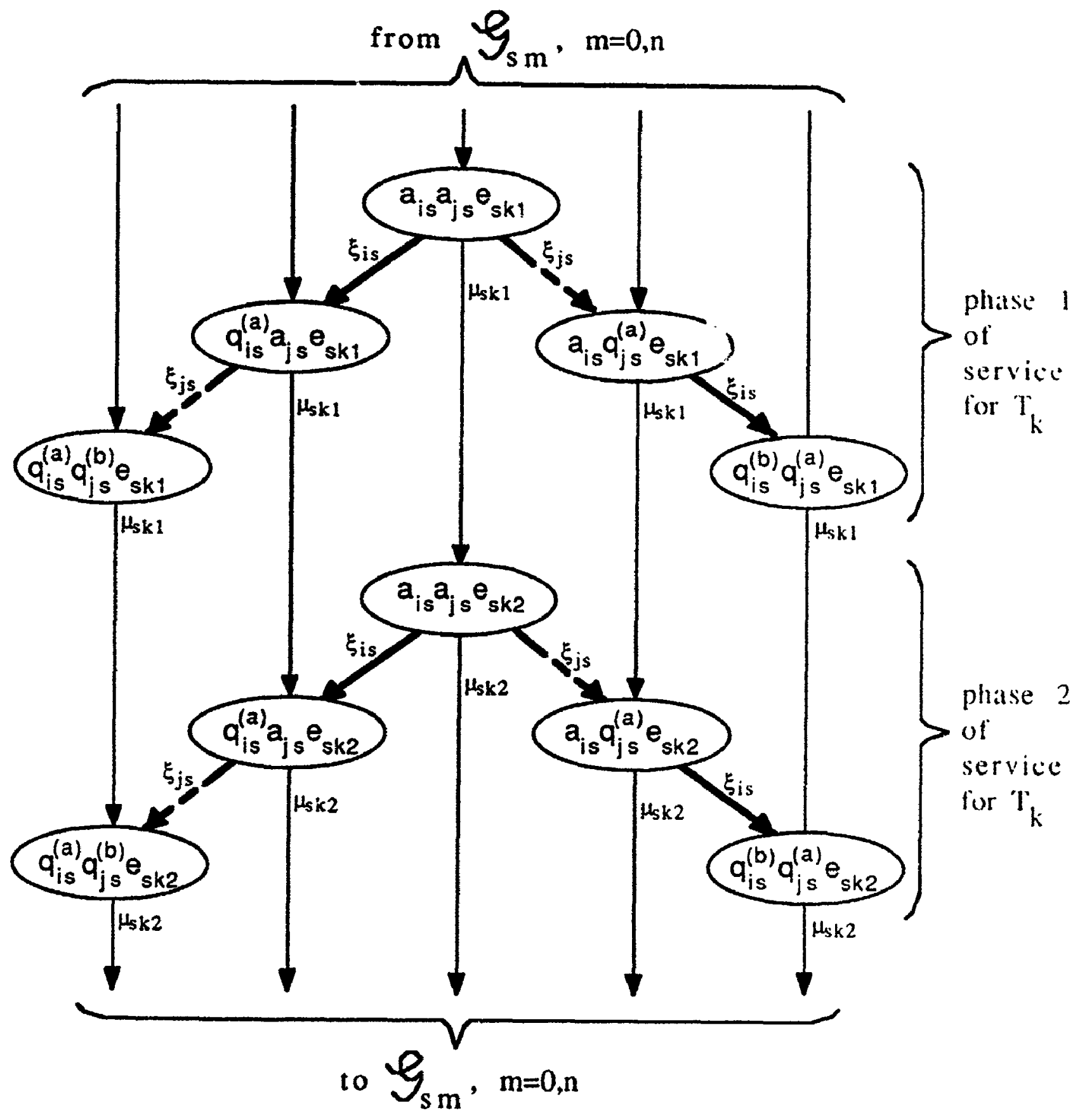

Figure 4.10: Group of aggregated states $\mathcal{G}_{s k}^{\prime \prime}$ where a non-designated task $T_{k}$ is in servicu. 
which is equivalent to:

$$
\frac{\mathcal{P}\left(a_{i,} e_{s k 1}\right)}{F_{k s,} \mu_{s k 1}^{-1}}=\frac{\mathcal{P}\left(a_{i s} a_{j,} e_{s k 1}\right)}{\mathcal{P}\left(a_{j,} e_{s k 1}\right)}
$$

From definition (4.54) for $A_{i \ell}^{k 1}$ and $A_{j,}^{k 1}$, we have:

$$
\mathcal{P}\left(a_{i,} e_{s k 1}\right)=\frac{F_{i s}}{\xi_{i s}} A_{i s}^{k 1}, \quad \mathcal{P}\left(a_{j,} e_{s k 1}\right)=\frac{F_{j s}}{\xi_{j s}} A_{j s}^{k 1}
$$

From (4.92) and (4.93) we can write the following expression:

$$
\mathcal{P}\left(a_{i s} a_{j s} e_{s k 1}\right)=\frac{\left(F_{i s} \xi_{i s}^{-1}\right)\left(F_{j s} \xi_{j s}^{-1}\right)}{F_{k s} \mu_{s k l}^{-1}} A_{i s}^{k 1} A_{j s}^{k 1}
$$

Equation (4.47) results from definition (4.90) for $C_{i s j}^{k 1}$ and (4.94).

The last equation (4.48) for $C_{i s j}^{k 2}$ is obtained from definition (4.90) and the following balance equation for state $\left(a_{i s} a_{j s} e_{s k 2}\right)$ in Figure 4.10:

$$
\mu_{s k 1}=\mathcal{P}\left(a_{i s} a_{j s} e_{s k 1}\right)=\left(\xi_{i s}+\xi_{j s}+\mu_{s k 2}\right) \mathcal{P}\left(a_{i s} a_{j s} e_{s k 2}\right) \square
$$

\subsection{Algorithm and experimental results}

\subsubsection{TDA/MVA(1) Algorithm}

Equations (4.39)-(4.48) represent a set of nonlinear equations that can be solved iteratively as follows:

Algorithm 1 TDA/MVA(1) algorithm for a simple client/server SRVN.

a) Initialize all $F_{i s}, B_{i s j}$ with some feasible values;

b) Compute new values for $A_{i s}^{i 2}$ (eq. 4.41), $A_{i s}^{j 1}$ (eq. 4.43), $A_{i \varepsilon}^{j 2}$ (eq. 4.42), $B_{i s j}^{k p}$ (eqs. 4.45, 4.46), $C_{i s j}^{k p}$ (eqs. 4.47, 4.48), $B_{i, j}$ (eq. 4.44); Update the arrival instant probabilities using an under-relaxation strategy, by applying only half of the change for each probability, i.e. prob $_{\text {new }}=0.5\left(\right.$ prob old + prob $\left._{\text {computed }}\right)$;

c) Determine a new value $w_{i s}\left(\right.$ eq. 4.40) and $F_{i s}$ (eq. 4.39);

d) Repeat steps b), c) until the total change in the arrival instant probabilities values is less than a given tolerance. 
The number of equations for the arrival instant probabilities used in step (b) of the algorithm depends on the number of clients $n$ as follows:

- $n$ equations of form (4.41)

- $n(n-1)$ equations of each of the forms (4.42)-(4.46)

- $n(n-1)(n-2)$ equations of each of the forms $(4.47),(4.48)$

The reduction in the order of computational complexity of the TDA/MVA(1) algorithm compared to the complexity of the exact solution is a consequence of the approximation used by the algorithm, which reduces the problem of building and solving a Markov chain with $O(n !)$ states to the problem of solving iteratively a system of $O\left(n^{3}\right)$ nonlinear equations.

The accuracy and convergence of the algorithm are assessed in the following subsection through experimental results.

\subsubsection{Experimental results}

TDA/MVA(1) algorithm has a much better accuracy than the previous heuristic MVA algorithm (see section 3.1.1) applied to the same class of systems. The accuracy of the algorithm was investigated by applying it to about two hundred cases, and comparing the results with the exact GSPN solutions. There were four test suites CS2, CS3, CS4, and CS5 with two, three, four, and five clients. Since the algorithm is derived from exact conditions for two clients and one server, the results for the test suite CS2 are exact (not shown here) while the results for the other test suites with more than two clients are approximate. The parameters and the results for test suites CS3, CS4, and CS5 are given in appendix B as follows: the model parameters in tables B.1, B.2, and B.3 respectively, and the results for reference throughputs in tables B.4, B.5, and B.6 respectively. The average relative throughput errors obtained with TDA/MVA(1) algorithm are compared in table 4.2 with the average errors obtained with the heuristic MVA algorithm applied to the same test suites.

The test suites were constructed "by hand", choosing the parameters to cover a wide range of cases (balanced systems and systems with various degree of unbalance, low and high utilization, etc.). Since a preliminary analysis of the experimental results suggested 
Table 4.2: Comparison of the mean absolute percentage error obtained with TDA/MVA(1) and Heuristic MVA algorithms for test suites CS3-CS5 (results given in appendix C)

\begin{tabular}{|c|cccc||}
\hline SRVN & \multicolumn{4}{|c||}{ Experimental test suites } \\
Method & CS2 & CS3 & CS4 & CS5 \\
\hline Heuristic MVA & $2.82 \%$ & $8.00 \%$ & $9.63 \%$ & $9.29 \%$ \\
TDA/MVA(1) & $0 \%$ & $0.46 \%$ & $0.53 \%$ & $0.65 \%$ \\
\hline
\end{tabular}

that the best approximations are obtained for models with balanced parameters, a "stress test" was designed for suite CS5, considering three "unbalance factors":

$r_{c}=$ client unbalance is the ratio between the longest and the shortest client execution time; $r_{s}=$ server unbalance, obtained as $\max \left(r_{s 1}, r_{s 2}\right)$, where $r_{s p}$ is the ratio between the longest and the shortest service time for phase $p$ among all server entries;

$r_{c s}=c l i e n t / s e r v e r$ unbalance is the maximum unbalance between the execution time of a client and the service time of the required entry (consider the phase giving the highest ratio)

The service times were chosen such that the three unbalance factors ranged between 1 and 100.

TDA/MVA(1) algorithm tends to overestimate the waiting (and thus underestimate the throughput) of those clients that are requesting the shortest server entry. In order to study the algorithm accuracy in function of model unbalance, the maximum error/case ME for test suite CS5 was plotted in function of the three "unbalance factors". (The maximum error/case $M E$ is the highest relative error in absolute value among the reference throughputs obtained for a given case). The analysis shows that client unbalance $r_{c}$ has no visible effect on the distribution of the maximum error/case, while the other two factors $r_{\text {a }}$ and $r_{c s}$ have some effect on it. The effect is more evident when considering the combined effect of server and client/server unbalance together, as in Figure 4.11, where the unbalance factor is computed as $\sqrt{r_{c s} r_{n} .}$ Table 4.3 shows that, in average, the maximum error/case grows with the unbalance of the model, but the effect is not very strong. 


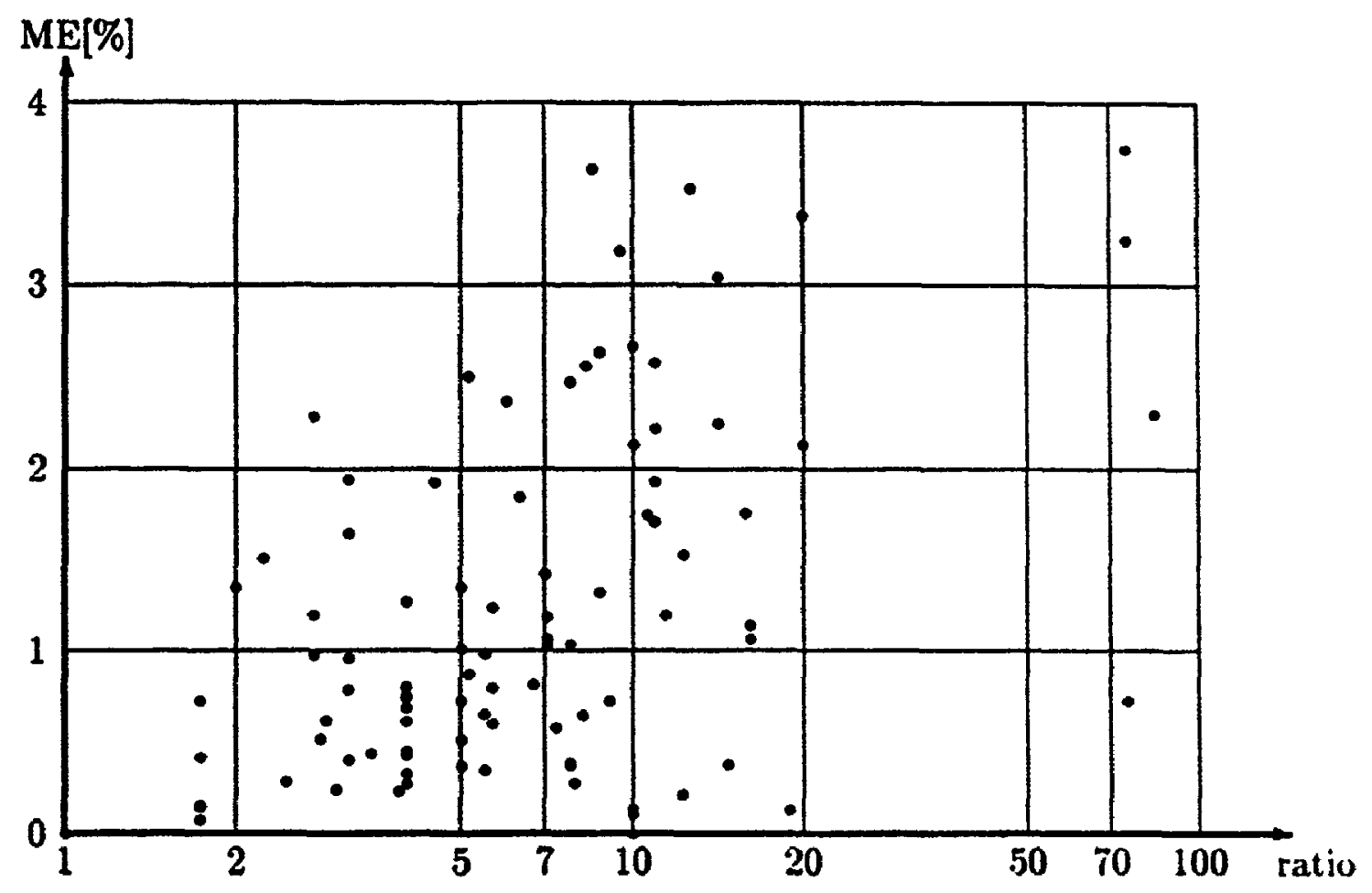

Figure 4.11: Maximum throughput error/case in function of client/server and server untal ance for test suite $\operatorname{CS5}\left(\right.$ ratio $\left.=\sqrt{r_{c s} r_{s}}\right)$

Table 4.3: TDA/MVA(1) Algorithm accuracy with respect to model unbalance

\begin{tabular}{|c||c|c|c||}
\hline & \multicolumn{3}{c|}{ Unbalance factor $r=\sqrt{r_{c a} r_{s}}$} \\
& $1 \leq r<5$ & $5 \leq r<10$ & $10 \leq r<100$ \\
\hline Number of cases & 35 & 33 & 27 \\
Avrg. of Max_err/case & $0.72 \%$ & $1.26 \%$ & $1.74 \%$ \\
\hline
\end{tabular}

Another experiment studied how the achieved server utilization affects the accuracy of the algorithm. A new test suite CS5_U was constructed from a "basic" case (number 5, , table B.6), with the worst accuracy in suite CS5 (the highest average error/case and ammon the highest $\mathrm{ME}$ ). Since the unbalance factors have also a strong impact on accuracy, thr cases from the new test suite CS5_U have the same server unbalance factor $r$. (by kereping, the same server execution times as in the basic case) and the same client unbalance fartor $r_{c}$ (by keeping the client service times proportional with the basic case, the proporticmality factor varying from 0.5 to 10 ). Figure 4.12 shows the dependency of the maximum error/cass. ME (defined above) on server utilization. There is a "peak" around 90\% utilization, where 
the worst ME is under $4 \%$.

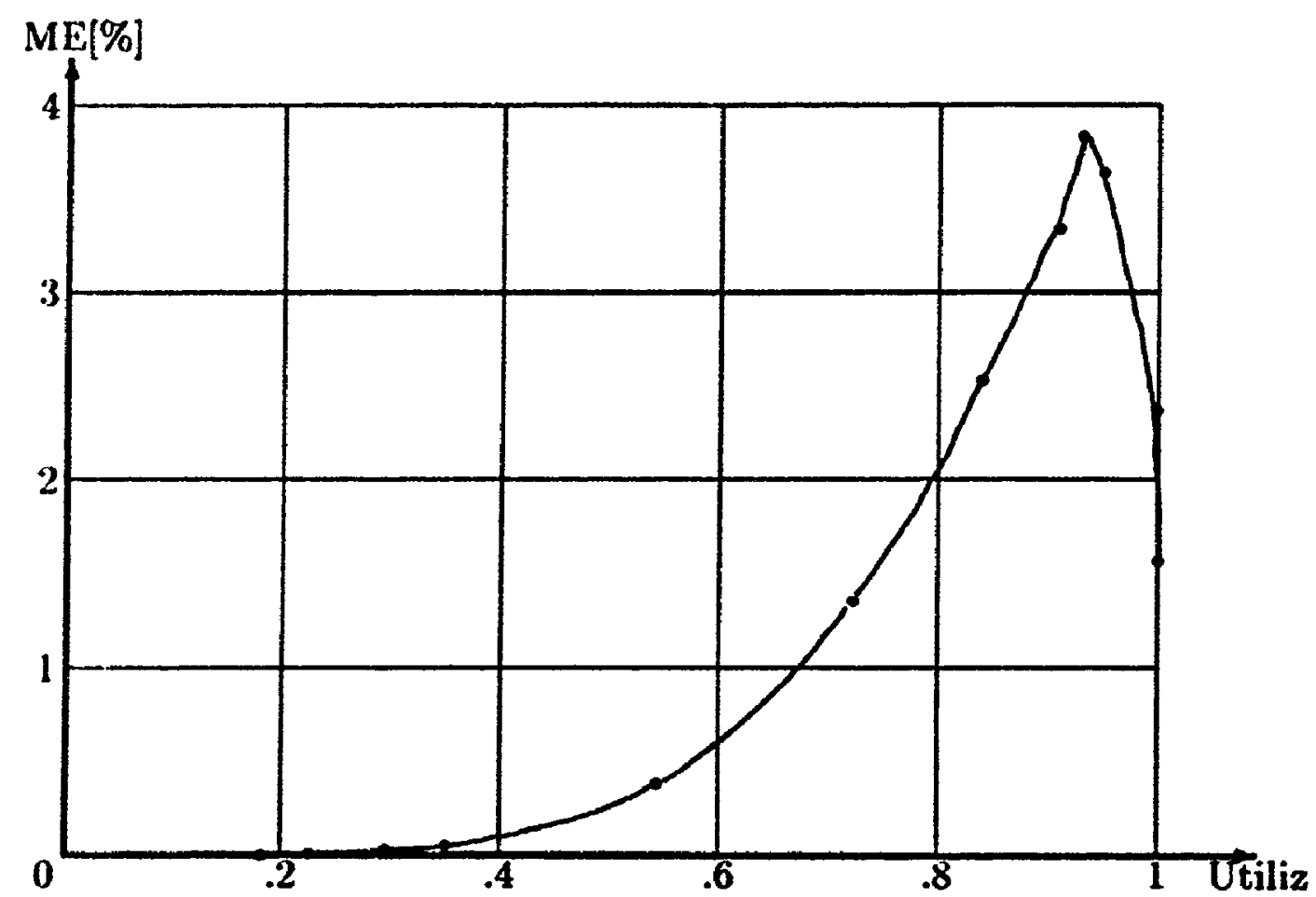

Figure 4.12: Maximum throughput error/case in function of server utilization for test suite CS5_U

The overall complexity of TDA/MVA(1) algorithm is dependent not only on the computational complexity of each iteration (which is $O\left(n^{3}\right)$ ), but also on the number of iterations required. It is quite difficult to predict the number of iterations necessary for the solution of a given SRVN model. Experiments have shown that the feasible values chosen for initialization in the first step of the algorithm do not have any impact on the final results. As the iteration progresses, the intermediate values for throughputs and arrival instant probabilities are no guaranteed to remain all the time feasible. However, the experiments have shown that they do converge toward feasible values. Some cases of oscillatory convergence have been observed for test cases with saturated server. From experience, the convergence is obtained quickly (a few dozen iterations) for the cases where the server is not saturated, but the number of iterations grows when the server approaches the saturation, and the model is unbalanced. (For balanced models, the number of iterations is low even if the utilization is very close to $100 \%$ ). 
The actual solution time experienced with TDA/MVA(1) algorithm was about 13 seconds in average for a case from test suite CS5 on a SUN 3/60 workstation. For a GSPN model of the same system on the same workstation, the time needed to obtain the exact solution with the GreatSPN package ([Chi87]) was about 30 times longer. 


\section{Chapter 5}

\section{Clients with Multi-class Requests in Simple Client/Server SRVN}

\subsection{Clients with multi-class requests}

In the previous chapter a simplifying assumption was made for simple SRVN client/server models, limiting to one the number of entries of a non-reference task that may be called directly by another task. In other words, the RV requests from a task $T_{i}$ to another task $T$, belong to a single class of requests, always seeing the same competitors, and therefore suffering the same mean RV delay (that is composed by queueing delay and service time). The fact that a task could not change the class of requests sent to another task does not restrict all customers arriving at a FIFO task queue to a single class of service. As seen in the previous chapter, every client was allowed to request a different server entry with different service time in every phase. This is similar to a multi-class (FIFO) queue in which customers cannot change class. However, the restriction is inappropriate when modelling software systems where a "software server" offers a range of services with different workload requirements to its clients, and a client needs several of this services. For example, an user of a data-base may send requests of different types (search, update, delete) to the server task that manages the access to the data-base, every of these requests being addressed to a different server entry.

In the present chapter the single-class restriction is removed allowing for client tasks with multi-class requests. The new algorithm TDA/MVA(2) proposed here accepts clients that change class by choosing randomly the server entry to which to send the next RV request. 


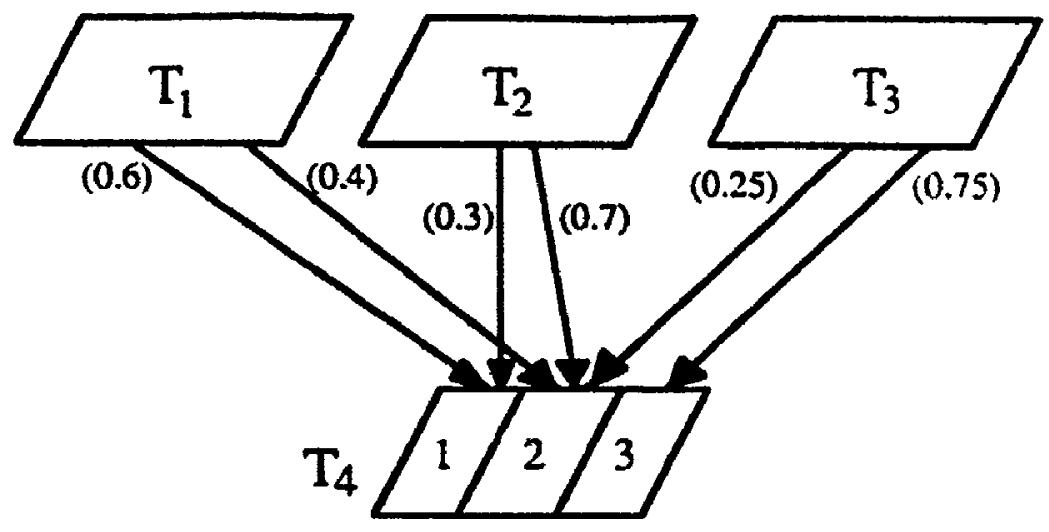

Figure 5.1: Example for test suite MR3

The requests from different classes require different service times, and thus suffer different mean RV delays. Request classes will be denoted by small letters at the beginning of the alphabet: $a, b, c, d$, etc. The set of all request classes from a task $T_{i}$ to a task $T_{s}$ is denoted by $\mathcal{R}_{i s}$, and the set of all request classes arriving at $T$, from different clients $T_{i}$ is $\mathcal{I}_{s}=U_{1} R_{t s}$.

The solution algorithm become more complex since the arrival instant probabilities are more numerous, being defined now for every request class ( $\mathcal{R}_{i s}$ ciasses for a client $T_{i}$ instead of one in the single-class case). Figures 5.1, 5.2 and 5.3 illustrate simple cases of SRVN models with one server and three, four, and five clients respectively, where some of the clients strnd multi-class requests to the server. The approximate results for this example will be discussed in section 5.3.2.

\subsubsection{Task Dependencies}

Although the most obvious reason for class changes is the choice of a different entry by the requesting task (that gives immediately a different service time, and thus a diffe, ent RV delay), it is also important to distinguish different classes even if the destination entry remains the same, because of task dependencies. In these cases the queueing component of the RV delay is different from class to class. The queueing delay depends on the state: of the requesting task (the entry and phase being executed when the request was made), and also on the state of the competing tasks. The RV communication imposes a strong intertask synchronization, and thus introduces dependencies between the competing tasks . 


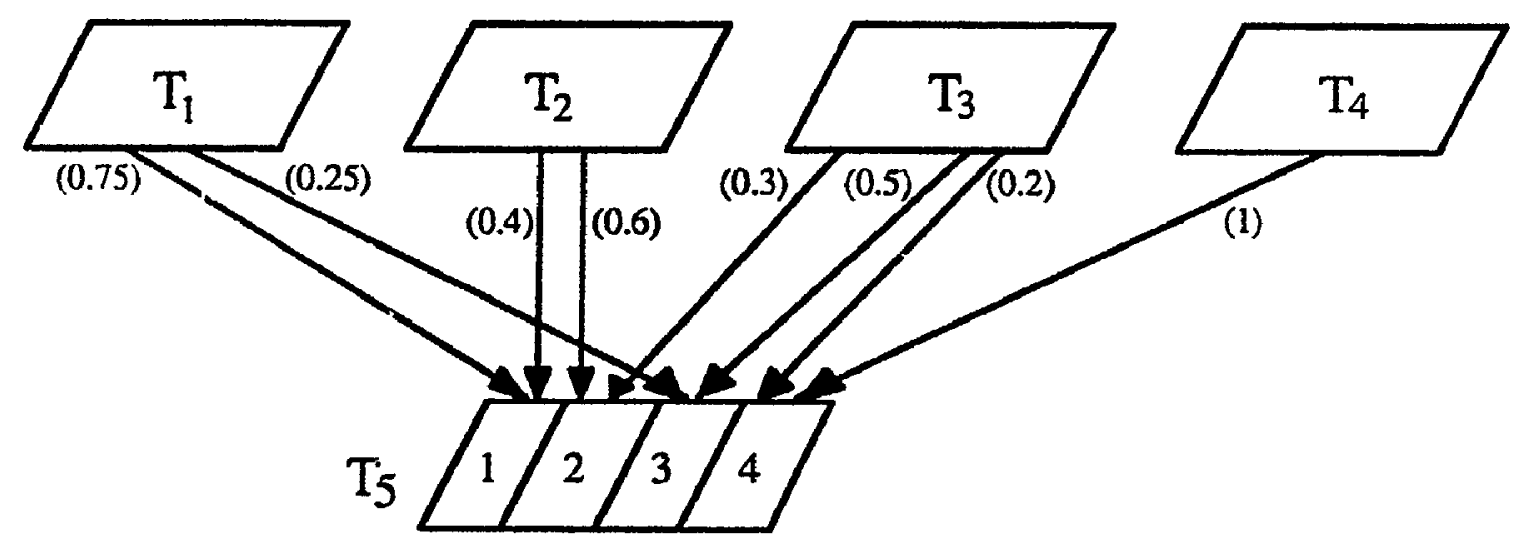

Figure 5.2: Example for test suite MR4

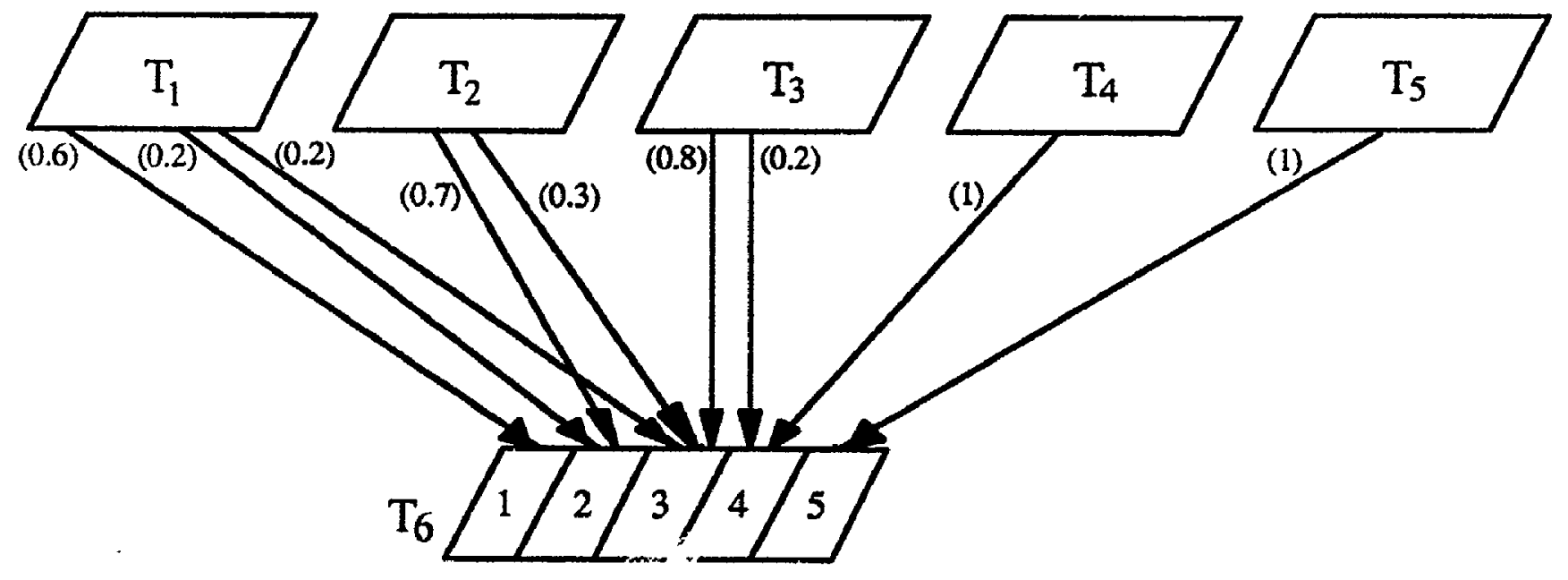

Figure 5.3: Example for test suite MR5 


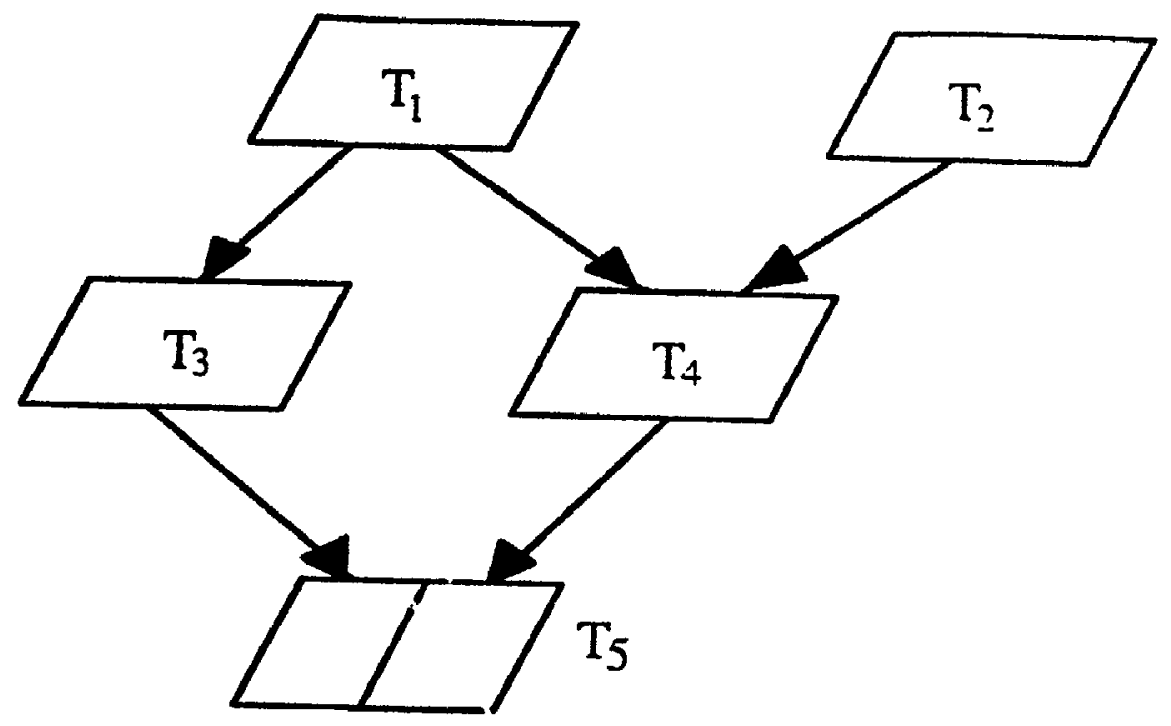

Figure 5.4: Example of an SRVN model with mutually exclusive requests

There are two main causes for task dependencies: the competing tasks share several common servers (as shown and dealt with in chapter 2), or they are activated by requests originated by a common source. The latter type of dependency has impact on the definition of request classes from one task to another. Figure 5.4 shows an example of SRVN system where two tasks $T_{3}$ and $T_{4}$ that compete for server $T_{5}$ are activated by requests generateri by a common source $T_{1}$. The requests originated by $T_{1}$ can arrive to $T_{5}$ by two different paths with nested RVs: $T_{1} \rightarrow T_{3} \rightarrow T_{5}$, or $T_{1} \rightarrow T_{4} \rightarrow T_{5}$. Due to SRVN task behaviour, the tasks on such paths can be blocked through a series of nested RVs, each of them waiting to be served by the following task on the path. The blocking process is dynamic, and may involve the whole path, or only a contiguous subset of path tasks (the intermediary tasks in a blocked subset must be in phase 1). If two or more paths have a common source, as in our example, then the source can be involved in a blocked subset only on one single path at a given moment. If, for example, $T_{1}$ is waiting for $T_{3}$, (that in turn may or may not be waiting for a nested RV with $T_{5}$ ), it is clear that $T_{1}$ cannot be waiting at the same time for a RV with $T_{4}$. Applying this property to the limit case when the blocked subset contains the whole path, two or more paths with a common source cannot be blocked simultaneously. Let us consider now the case where two $\mathrm{rr}$ more paths with a common source are interserting 
each other at a common server (as happen at $T_{5}$ in Figure 5.4). Each path contains one task competing directly for the common server, the last before the intersection $\left(T_{3}\right.$ and $T_{4}$ in our example). By the property shown above, the requests for the common server made by these tasks in phase 1 when the whole path is blocked are mutually exclusive. Thus, request for $T_{5}$ made by $T_{4}$ in phase 1 as a result of a request from $T_{1}$ cannot see in service or in queue a request for $T_{5}$ made by $T_{3}$ in phase 1 . On the other hand, a request made by $T_{4}$ in phase 1 as a result of a request from $T_{2}$ can compete with any requests from $T_{3}$ to $T_{5}$.

From this example we can see that the request class depends on the state (entry and phase) of the task making the request, on the destination entry of the server, and also on the path through which the request was originated. This gives a recursive definition for request classes as follows:

A request class from a task $T_{i}$ to a task $T_{3}$ is characterized by:

- the entry and phase of $T_{i}$ from where the request is sent to $T_{\text {. }}$

- the destination entry of $T$,

- the request class served by $T_{i}$ when the request to $T_{s}$ is sent (only if $T_{i}$ is a non-reference task).

The following section extends TDA/MVA(1) algorithm proposed in chapter 4 , to the case where the clients make independent multi-class requests to the server. Section 5.3 .2 presents test cases and discusses the experimental results.

TDA/MVA(1) algorithm introduced for clients with single-class requests, and TDA/MVA(2) algorithm for clients with multi-class requests will be integrated in chapter 6 into a decomposition solution method for complex SRVN models with any number of accepting tasks. An SRVN model will be decomposed into a set of interrelated client/server submodels, that will be solved iteratively. Although dealing with multi-class requests from a client, the decomposition algorithm proposed in this thesis will not take into consideration the path through which a request was originated in establishing the class of requests; only the choice of a different server entry by a client will produce a class change. This is a limitation that should be addressed in future research. 


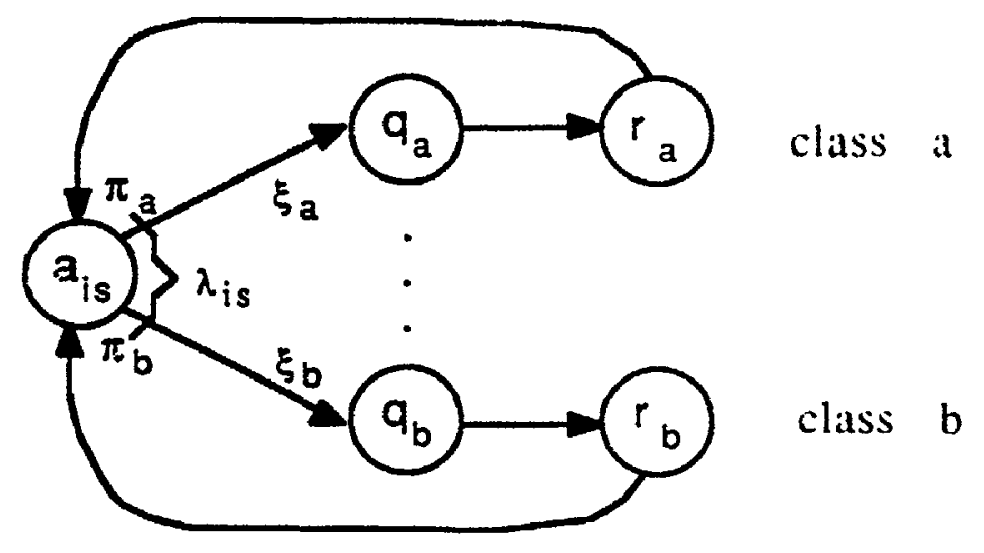

Figure 5.5: A bstract representation of a client with multi-class requests

\subsection{Arrival probabilities for clients with multi-class :equests}

This sections extends the algorithm for arrival instant probabilities introduced in the previous chapter for simple client/server SRVN where a client was allowed only single-class requests to the case where a client can send multi-class requests to the server.

The abstract representation for a client given in Figure 5.5, extends the representation used in chapter 4 for clients with single class requests, by introducing different que ueing and = srvi st st es for different request classes ( $q_{c}$ and $r_{c}$ respectively, for $\left.c \in \mathcal{R}_{i s}\right)$. As in the sing:-4as representation, $a_{i s}$ is an aggregated state of task $T_{i}$ obtained by TDA-2 including all ine :.rates where $T_{i}$ is not interacting directly with the server (for more details see section 6. ?. ' 1 time spent in state $a_{i s}$ is assumed to be exponentially distributed with known rate? $\lambda_{i s}$. Brride $\lambda_{i s}$, the parameters of the client/server model include for a client $T_{i}$ the choire probabilities $\pi_{c}$ for all request classes $c \in \mathcal{R}_{\text {is. }}$.

$i$ he mean rate $\xi_{c}$ for a given request class is determined as:

$$
\xi_{c}=\pi_{c} \lambda_{i s}
$$

Th: MVA equation (4.39) used to determine the frequency $F_{i s}$ of requests from $T_{i}$ to $T$, in the single class case becomes for the multi-class case:

$$
F_{i s}=\frac{1}{\lambda_{i s}^{-1}+\sum_{c \in \mathcal{R}_{i s}} \pi_{c}\left(w_{c s}+x_{s g 1}\right)}
$$




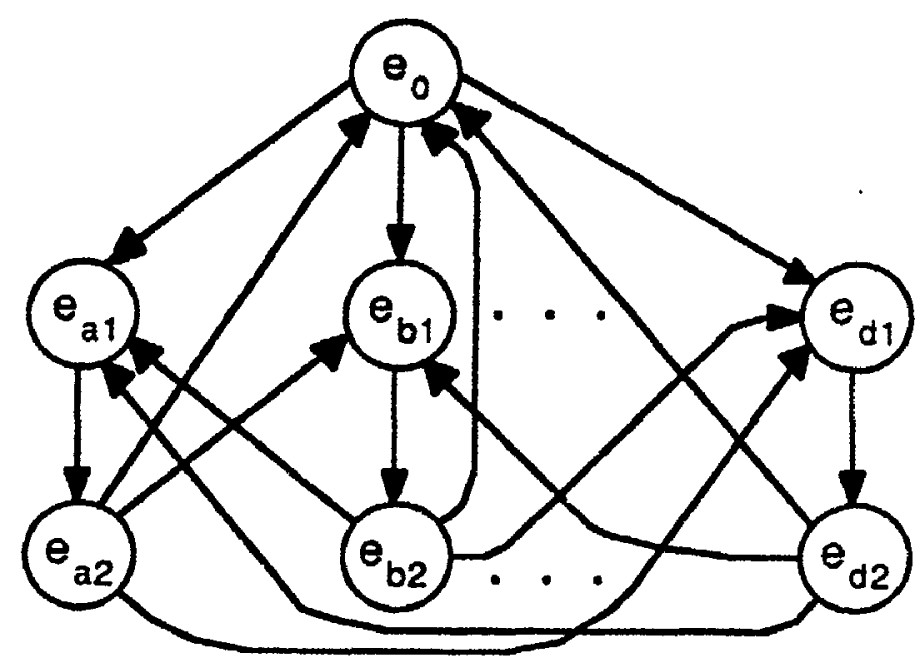

Figure 5.6: Abstract representation of a server

where:

$w_{c s}=$ the mean queueing delay for a class $c$ request

$x_{2 g 1}=$ the mean service time at server $s$ for a class $c$ request sent to entry $g$ of the server (exponentially distributed with rate $\mu_{c}$ ).

The request frequency for a class $c$ for any $c \in \mathcal{R}_{i s}$ is determined as:

$$
F_{c}=\pi_{c} F_{i s}
$$

The abstract representation for the server given in Figure 5.6 contains two states $e_{c 1}, e_{c 2}$ for the phase 1 and 2 of service given by $T_{s}$ for every request class $c \in \mathcal{I}_{s}$. State $e_{0}$ is the idle state of the server, where the input queue is empty. In the client/server SRVN, the time spent in every state $e_{c p}$ is assumed exponentially distributed with known rate $\mu_{c p}$.

The MC for a simple client/server SRVN with clients with multi-class requests is an extension of the MC for the single-class case described in section 4.2; however, the state vector has the same number of components, i.e. one for every task. The states can be lumped into $\left|\mathcal{I}_{\mathfrak{l}}\right|+1$ groups as in Figure 5.7, where a group $\mathcal{G}_{\mathrm{cs}}$ contains all the states where $T$, is serving class $c$ requests. There are several groups corresponding to each client task, while in the case with single-class requests (see Figure 4.4) there is a single group $\mathcal{G}_{i}$, per 


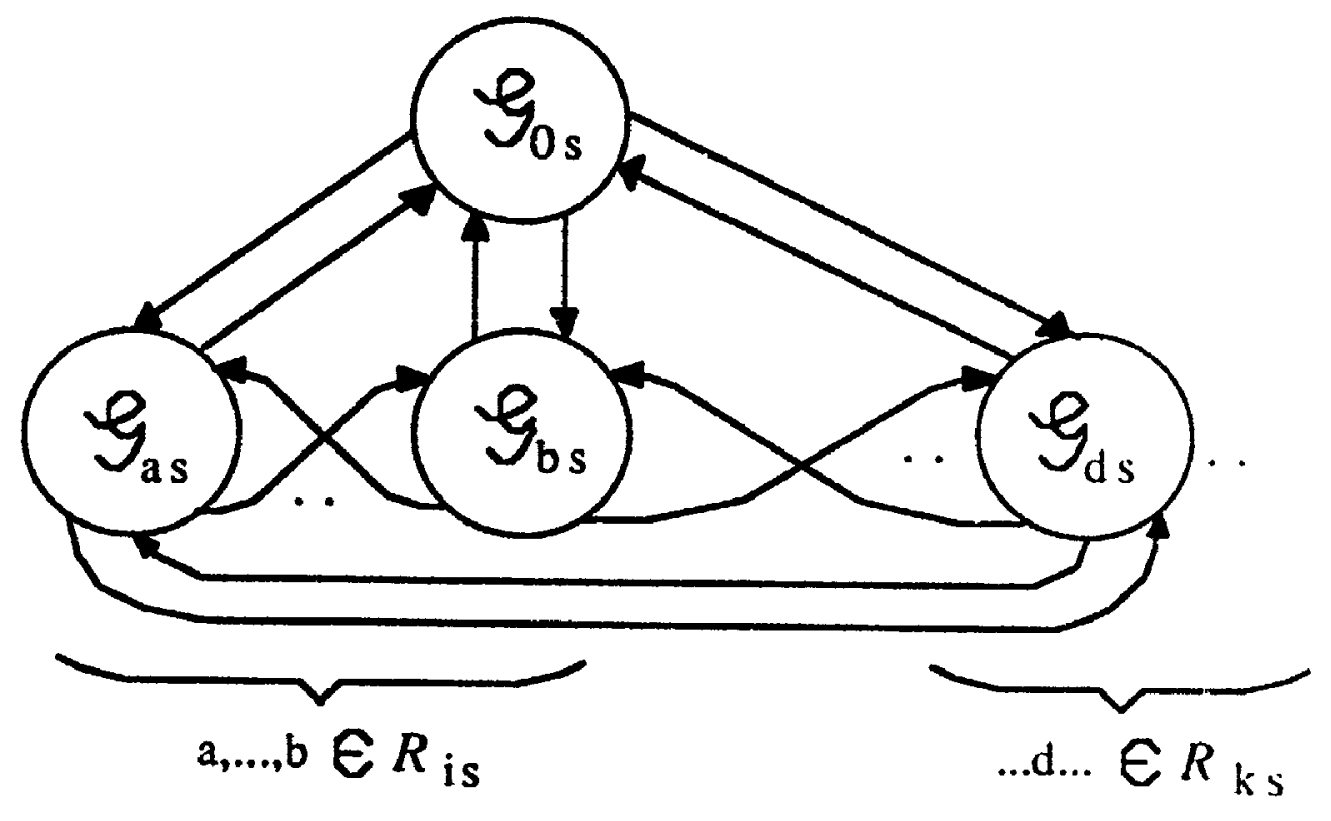

Figure 5.7: Groups of MC states corresponding to different request classes

client. There is also a difference inside the groups due to the fact that the request arrivals, queueing and service are differentiated by class. Figure 5.8 shows the structure of a group $\mathcal{G}_{c \text {, with }} c \in \mathcal{R}_{j}$ that is a part of a non-aggregated MC for $n=2$, or of an aggregated MC: for $n>2$, obtained by TDA-1 for the set of designated tasks $\{i, j, s\}$. The proofs that the aggregated MCs for a given set of designated tasks have the same structure independent of the number of clients is similar to the proofs for the single-class case given in section 4.3, and thus will not be given.

The notation for the arrival instant probabilities defined in chapter 4 is adapted to the case with multi-class requests by substituting the request class in place of the client and server identifiers, as follows:

$A_{a}^{b p}$ is the probability that a class $a$ request arriving to $T$, finds the server busy in phase $p$, serving a class $b$ request

$A_{a}^{0}$ is the probability that a class $a$ request finds the server idle

$B_{a b}$ is the probability that a class $a$ RV request arriving to $T_{s}$ finds a class $b$ request waiting 


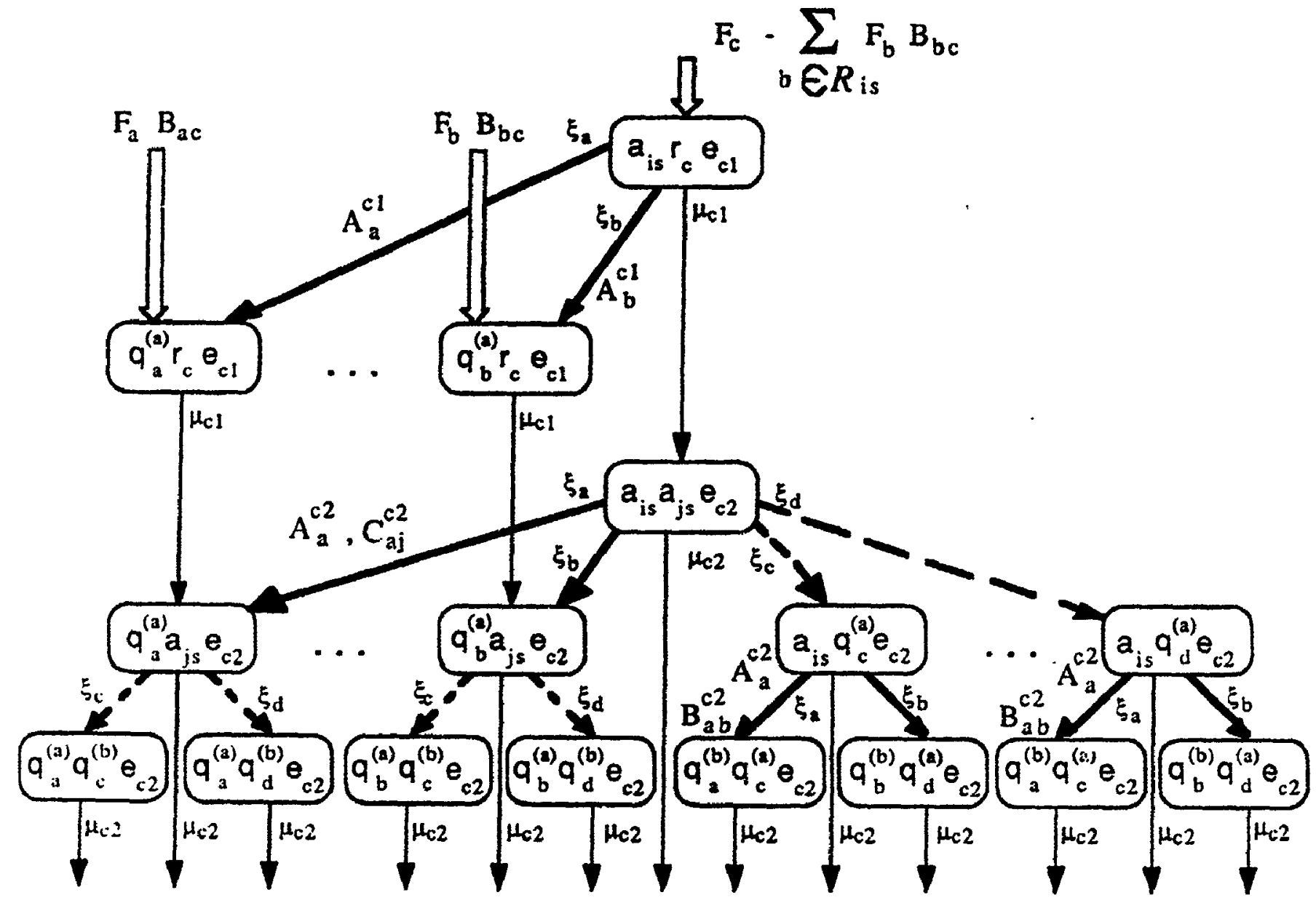

Figure 5.8: Group $\mathcal{G}_{c s}$ of $\mathrm{MC}$ states aggregated for $D=\{i, j, s\}$, where $a, b \in \mathcal{R}_{i s}, \quad c, d \in$ $\mathcal{R}_{j_{\Perp}}$, and $c$ from $T_{j}$ is in service 
in queue (not in service)

$C_{a j}$ is the probability that a class $a$ request from $T_{i}$ arriving to $T_{\mathrm{s}}$ does not see any request from $T_{j}$ in queue or in service.

From the definition of arrival probabilities, and from the fact that the request classes from the same task are mutually exclusive, we have the following relations:

$$
\begin{gathered}
\sum_{b \in \mathcal{I}_{*}} A_{a}^{b p}+A_{a}^{0}=1 \\
\sum_{b \in \mathcal{R}_{j,}} A_{a}^{b 1}+\sum_{b \in \mathcal{R}, j} B_{a b}+C_{a j}=1
\end{gathered}
$$

When obtaining the equations for arrival instant probabilities we need to decompose further the probabilities $B$ and $C$ in function of the server states:

$B_{a b}^{c p}$ is the probability that a class $a$ request arriving to $T$, when $T$, is serving $c$ in phase $p$, finds a class $b$ request waiting in queue (not in service)

$C_{a j}^{C P}$ is the probability that a class $a$ request from $T_{i}$ arriving when $T_{a}$ is serving $c$ in phase $p$ does not see any request from $T_{j}$ in queue or in service.

The queueing delay $w_{a s}$ of a class $a$ request sent to entry $g$ of the server is computed as follows:

$$
w_{a s}=\sum_{b \in \mathcal{I}_{s}}\left[A_{a}^{b 1}\left(x_{s g 1}+x_{s g 2}\right)+A_{a}^{b 2} x_{s g 2}+B_{a b}\left(x_{s g 1}+x_{s g 2}\right)\right]
$$

\section{Overtaking probabilities}

It is known that a request $a$ from $T_{i}$ may not arrive when another request from the same. task is in service in phase 1 , since $T_{i}$ is blocked for the RV duration. Thus:

$$
A_{a}^{b 1}=0 \quad \text { for } a, b \in \mathcal{R}_{\text {is }}
$$

However, a request $a$ from a task $T_{i}$ may find the server executing the second phase for a request $b$ from the same task. Similarly to the equation (4.41) from the previous chapter, the overtaking probabilities $A_{a}^{b 2}$ are determined from an aggregated MC where only $T_{i}$ and 


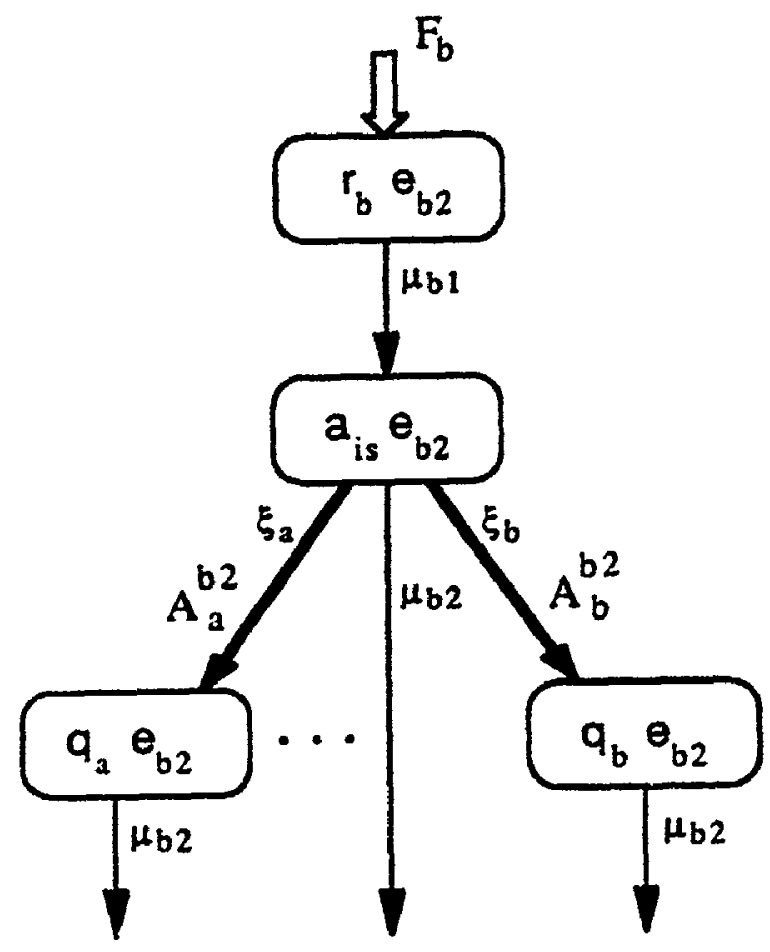

Figure 5.9: Group $\mathcal{G}_{b}$, of $\mathrm{MC}$ states aggregated for $D=\{i, s\}$, where $a, b \in \mathcal{R}_{i s}$, and $b$ from $T_{i}$ is in service

$T_{s}$ are designated tasks. Figure 5.9 shows such an aggregation for the group of states $\mathcal{G}_{b s}$ where $b \in \mathcal{R}_{i,}$ is in service. The arrival probability $A_{a}^{b 2}$ is obtained from the following ratio of flows:

$$
A_{a}^{62}=\frac{\xi_{a} \mathcal{P}\left(a_{i s} e_{b 2}\right)}{F_{a}}
$$

From the balance equations of the aggregated MC from Figure 5.9 we have:

$$
\mathcal{P}\left(a_{i,} e_{b 2}\right)=F_{b} /\left(\lambda_{i s}+\mu_{b 2}\right)
$$

From the last two relations it results:

$$
A_{a}^{b 2}=\frac{\pi_{a}}{\pi_{b}} \frac{\xi_{a}}{\lambda_{i s}+\mu_{b 2}} \quad \text { for } a, b \in \mathcal{R}_{i s}
$$

Arrival when other task is in service

As in the case for the single-class case treated in chapter 4 , there is a relation between the arrival probabilities $A_{a}^{c 1}$ and $A_{a}^{c 2}$, where classes $a$ and $c$ criginate from different tasks, that 


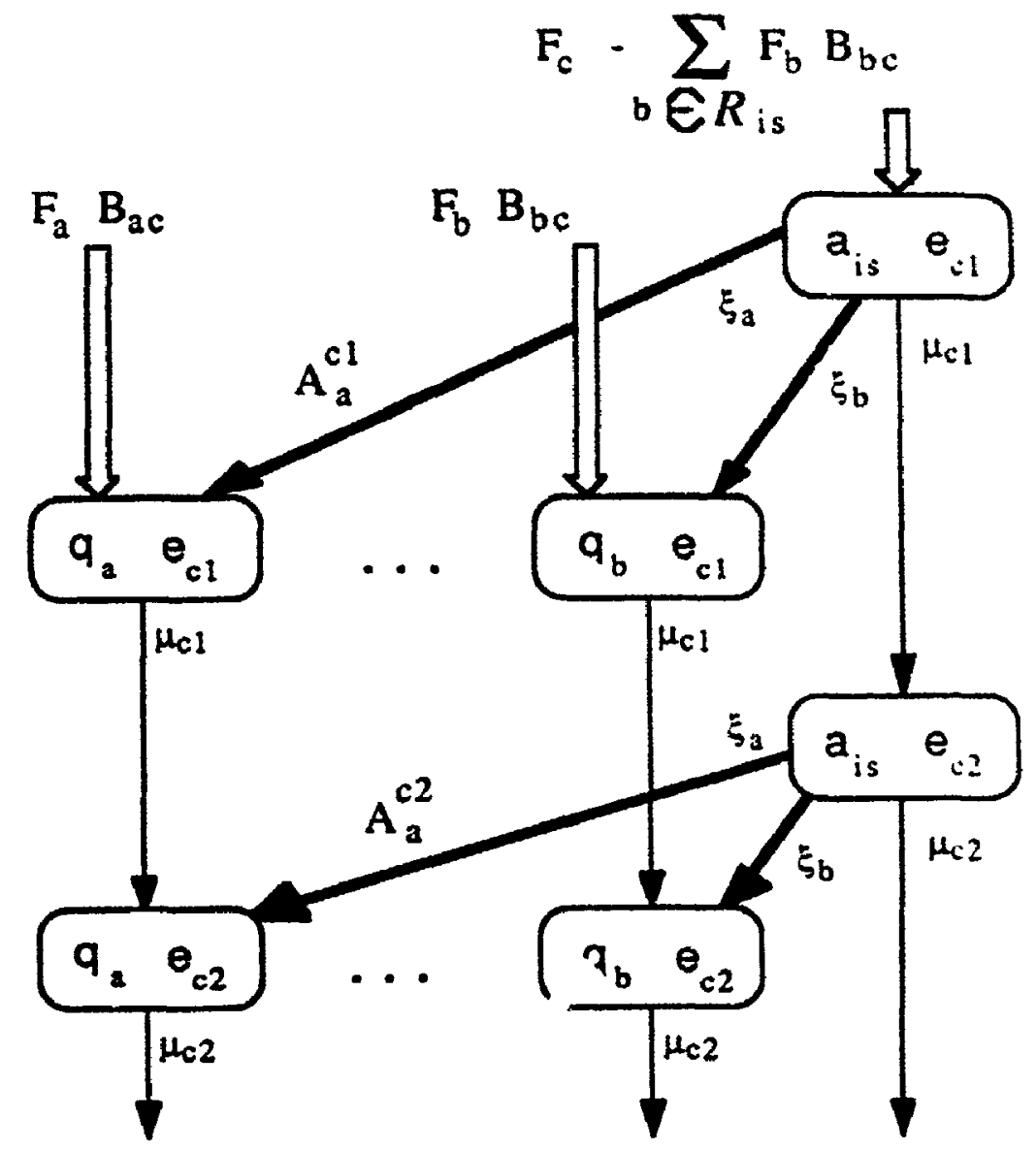

Figure 5.10: Group $\mathcal{G}_{c,}$ of $\mathrm{MC}$ states aggregated for $D=\{i, s\}$, where $a \in \mathcal{R}_{\mathrm{s}}, c \in \mathcal{R}_{\mathrm{s}}$, and $c$ from $T_{j}$ is in service

shows that these probabilities are not proportional with the utilizations of server phasess in which the arrivals occur.

The arrival probabilities of interest are determined from the aggregated MC with $T_{2}, T_{s}$ as designated tasks. Figure 5.10 shows such an aggregation for the group of states $\mathcal{G}_{c s}$ where $c$ is served. The probabilities $A_{a}^{c 1}$ and $A_{a}^{c 2}$ are obtained as:

$$
A_{a}^{c 1}=\frac{\xi_{a} \mathcal{P}\left(a_{i,} e_{c 1}\right)}{F_{a}} \quad A_{a}^{c 2}=\frac{\xi_{a} \mathcal{P}\left(a_{i,} e_{c a}\right)}{F_{a}}
$$

From the balance equations of the aggregated MC we have:

$$
\mathcal{P}\left(a_{i s} e_{c 2}\right)=\frac{\mu_{c 1} \mathcal{P}\left(a_{i s} e_{c 1}\right)}{\left(\lambda_{i s}+\mu_{c 2}\right)}
$$


From the last expression a relation similar to (4.42) is obtained:

$$
A_{a}^{c 2}=\frac{\mu_{c 1}}{\lambda_{i s}+\mu_{c 2}} A_{a}^{c 1} \quad \text { for } a \in \mathcal{R}_{i s}, c \in \mathcal{R}_{j},
$$

The utilizations of server phases in which the arrival occurs are $U_{c 1}=F_{c} \mu_{c 1}^{-1}$ and $U_{c 2}=F_{c} \mu_{c 2}^{-1}$ respectively. It results from the previous relation that:

$$
\frac{A_{a}^{c 1}}{A_{a}^{c 2}} \neq \frac{U_{c 1}}{U_{c 2}}
$$

Relation between probabilities to find another request in service or in queue

Equation (4.43) shows a simple relation between the arrival probabilities from a task that finds another task in service or in queue, and the frequency of requests from the two tasks. The corresponding relation for the multi-class case is determined here. The aggregated MC that is used represents the states of three tasks: $\{i, j, s\}$. Figure 5.8 shows such an aggregation for the group $\mathcal{G}_{c,}$ of MC states where $c \in \boldsymbol{R}_{j,}$ is in service.

The transition flow from the state $\left(a_{i s} r_{c} e_{c 1}\right)$ to $\left(q_{a} r_{c} e_{c 1}\right)$ represents the fraction of the class- $a$ flow $F_{a}$ that finds $c$ in service (i.e. $F_{a} A_{a}^{\text {cl }}$ ). Due to the FIFO scheduling policy for the server queue, all class $a$ requests that find $c$ in queue will be still in queue when the service for $c$ begins. In terms of MC states and transitions, this means that the fraction of $F_{a}$ flow that finds $c$ in queue (i.e. $\left.F_{a} B_{a c}\right)$ represents the input flow to the state $\left(q_{a} r_{c} e_{c 1}\right)$ from all the states where request $c$ is not served in first phase. Since the sum of input flows to all MC states where $c$ is served in first phase from all the other MC state where $c$ is not served in first phase is exactly the class- $c$ flow $F_{c}$, it results that the input flow to state $\left(a_{i s} r_{c} e_{c 1}\right)$ is given by:

$$
\Phi\left(a_{i s} r_{c} e_{c 1}\right)=F_{c}-\sum_{a \in \mathcal{R}_{i s}} F_{a} B_{a c}
$$

Thus we can write:

$$
\mathcal{P}\left(a_{i s} r_{c} e_{c 1}\right)=\frac{F_{c}-\sum_{a \in \mathcal{R}} \mathcal{R}_{i s} F_{a} B_{a c}}{\mu_{c 1}+\lambda_{i s}}
$$

From the MC given in Figure 5.8 we have:

$$
\Phi\left(q_{a} r_{c} e_{c 1}\right)=F_{a}\left(B_{a c}+A_{a}^{c 1}\right)
$$

The probability $A_{a}^{c 1}$ that $a$ finds $c$ in service is given by:

$$
A_{a}^{c 1}=\frac{\xi_{a}}{F_{a}} \mathcal{P}\left(a_{i s} r_{c} e_{c 1}\right)
$$


From (5.9) and (5.11) then results the following relation between the arrival probabilities that a request $a$ from one client finds the request $c$ from another client in queue or in service, and the frequencies of these requests:

$$
\frac{\mu_{c 1}+\lambda_{i s}}{\xi_{a}} A_{a}^{c 1} F_{a}+\sum_{b \in \mathcal{R}_{i s}} F_{b} B_{b c}=F_{c} \quad \text { for } a, b \in \mathcal{R}_{i s,}, c \in \mathcal{R}_{j s}
$$

\section{Probability to find another request in queue}

Let $T_{i}$ and $T_{j}$ be two clients such that a class $a$ of requests comes from $T_{i}$ and a class $d$ comes from $T_{j}$. The probability $B_{a d}$ is determined from its components that depend on server states:

$$
B_{a d}=\sum_{c \in \mathcal{I}_{a}} \sum_{p} B_{a d}^{c p} \quad \text { for } a \in \mathcal{R}_{i s}, \quad b \in \mathcal{R}_{j},
$$

We are using the aggregated $\mathrm{MC}$ with $\{i, j, s\}$ as designated tasks . A component $B_{u d i}^{c p}$ is determined differently if the request $c$ executed by the server when $a$ arrives belongs to $T$, to $T_{j}$, or to another task $T_{k}, k \neq i, j$.

Case 1: The server executes a request $c$ from the same task that generates the arriving

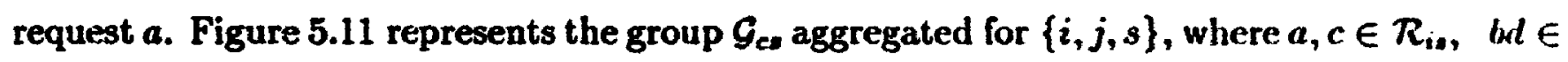
$\boldsymbol{R}_{\text {js. }}$

Any request arrival from $T_{i}$ is impossible in the first phase of service for $c$, since $T$, is biccked until the RV ends. Thus we have $B_{a d}^{c 1}=0$.

For the second phase of service, we can write:

$$
B_{a d}^{c 2}=\frac{\xi_{a} \mathcal{P}\left(a_{i s} q_{d} e_{c 2}\right)}{F_{a}}
$$

From the MC balance equations we get:

$$
\mathcal{P}\left(a_{i s} q_{d} e_{c 2}\right)=\frac{\xi_{d} \mathcal{P}\left(a_{i s} a_{j s} e_{c 2}\right)+\mu_{c 1} \mathcal{P}\left(r_{c} q_{d} e_{c 1}\right)}{\mu_{c 2}+\lambda_{i s}}
$$

The first state probability on the right side of (5.15) is used in the definition of probability $C_{a j}^{c 2}$ as follows:

$$
C_{a j}^{c 2}=\frac{\xi_{a} \mathcal{P}\left(a_{i s} a_{j,} e_{c 2}\right)}{F_{a}}
$$




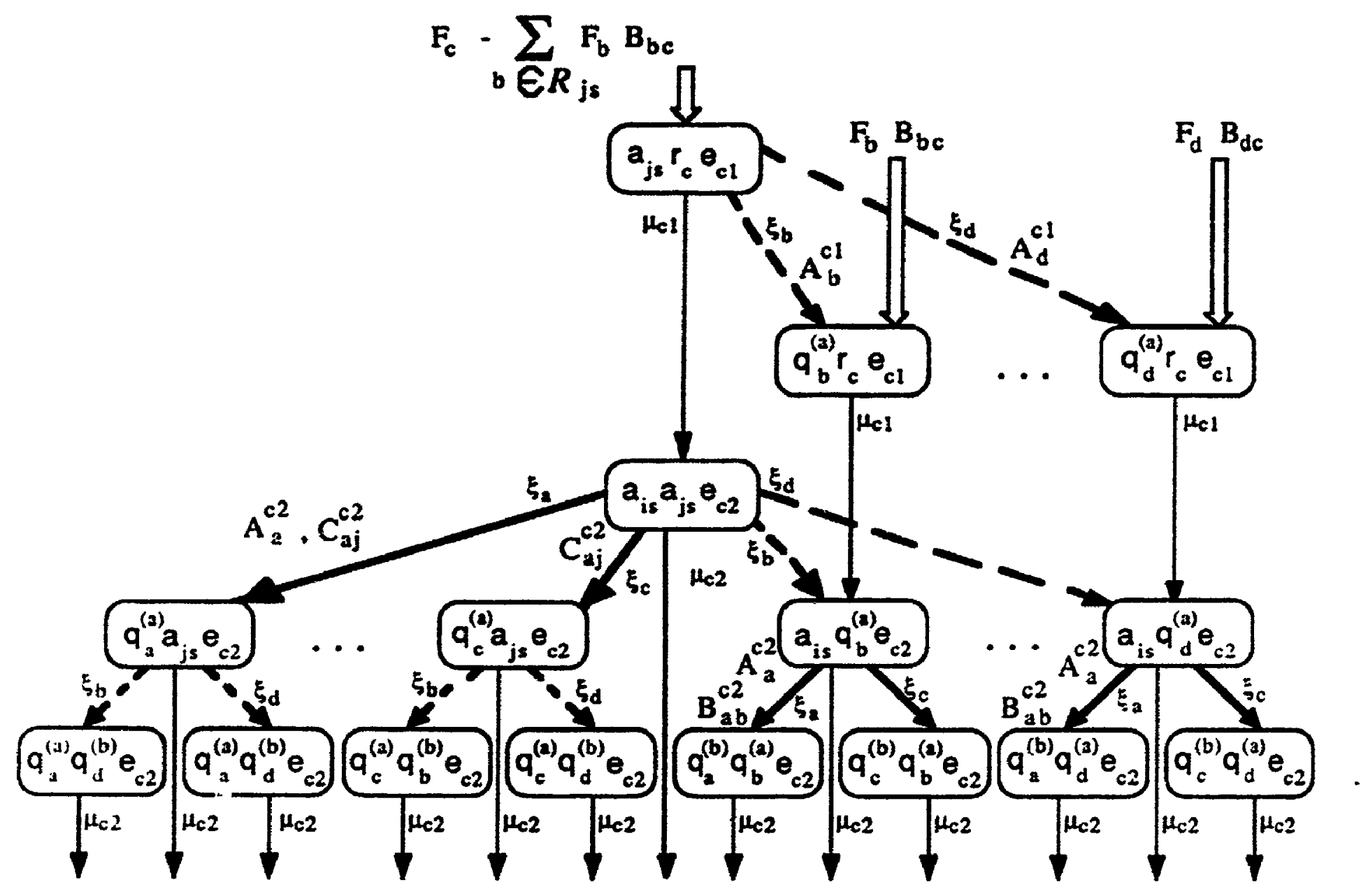

Figure 5.11: Group $\mathcal{G}_{c s}$ of MC states aggregated for $D=\{i, j, s\}$, where $a, c \in \mathcal{R}_{i s}, b, d \in$ $\mathcal{R}_{\text {ges }}$, and $c$ from $T_{i}$ is in service 
The second state probability on the right side of (5.15) can be written by using a relation similar to (5.10):

$$
\mathcal{P}\left(r_{c} q_{d} e_{c 1}\right)=\frac{F_{d}\left(B_{d c}+A_{d}^{c \mathrm{I}}\right)}{\mu_{c 1}}
$$

From (5.14) - (5.17) we get:

$$
B_{a d}^{c 2}=\frac{\xi_{c}}{\mu_{c 2}+\lambda_{i s}} C_{a j}^{c 2}+\frac{c_{a}}{\mu_{c 2}}+\lambda_{i s} \cdot \frac{F_{d}\left(B_{b c}+A_{d}^{c l}\right)}{F_{a}} \quad \text { for } a, c \in \mathcal{R}_{i s}, d \in \mathcal{R}_{, s}
$$

We determine now the probability $C_{a j}^{c 2}$ by using (5.16). From the MC balance equations we have:

$$
\mathcal{P}\left(a_{i,}, a_{j,} e_{c 2}\right)=\frac{\mu_{c 1}}{\mu_{c 2}+\lambda_{i s}+\lambda_{j,}} \mathcal{P}\left(r_{c} a_{j,} e_{c 1}\right)
$$

From $(5,16),(5.19)$, and by using a relation similar to (5.9) for $\mathcal{P}\left(r_{\mathrm{c}} a_{j,} e_{\mathrm{c} 1}\right)$ we obtain:

$$
C_{a j}^{c 2}=\frac{\xi_{a} \mu_{c 1}}{\left(\mu_{c 1}+\lambda_{j s}\right)\left(\mu_{c 2}+\lambda_{i s}+\lambda_{j a}\right)} \cdot \frac{F_{c}-\sum_{b \in \mathcal{R}_{j s}} F_{b} B_{b c}}{F_{a}} \quad \text { for } a, c \in \mathcal{R}_{s s}
$$

Case 2: The server executes a request $c$ that was sent by the same task $T$, as the request $d$ found in queue by the arriving request $a$ coming from $T_{i}$. Figure 5.8 represents the group

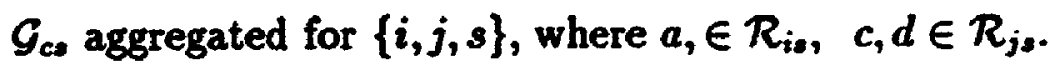

In the first phase of service for $c$ no other request from the same task $T_{j}$ can be found at the server, since $T_{j}$ is blocked until the end of RV. Thus we have $B_{a d}^{c l}=0$.

For the second phase of service, $B_{a d}^{c 2}$ is obtained from (5.14) as the ratio between the flow of requests $a$ arriving when $T_{j}$ is in state $q_{d}$ and $T_{\text {, in state }} e_{\mathrm{c} 2}$, and the total flow $F_{\mathrm{a}}$. From the $\mathrm{MC}$ balance equations we get:

$$
P\left(a_{i s} q_{d} e_{c 2}\right)=\frac{\xi_{d} \mathcal{P}\left(a_{i s} a_{j s} e_{c 2}\right)}{\mu_{c 2}+\lambda_{i s}}
$$

The state probability on the right side of $(5.21)$ is used in the definition of probability $C_{a j}^{\prime 2}$ as shown by (5.16). From (5.14), (5.21), and (5.16) we get:

$$
B_{a d}^{c 2}=\frac{\xi_{c}}{\mu_{c 2}+\lambda_{i s}} C_{a j}^{c 2} \text { for } a \in \mathcal{R}_{i s}, c, d \in \mathcal{R}_{j s}
$$

The probability $C_{a j}^{c 2}$ defined by (5.16) is determined now in function of other arrival probabilities. We use the following MC balance equations similar to (5.19):

$$
\mathcal{P}\left(a_{i s} a_{j,} e_{c 2}\right)=\frac{\mu_{c 1}}{\mu_{c 2}+\lambda_{i s}+\lambda_{j s}} \mathcal{P}\left(a_{i s} r_{c} e_{c 1}\right)
$$




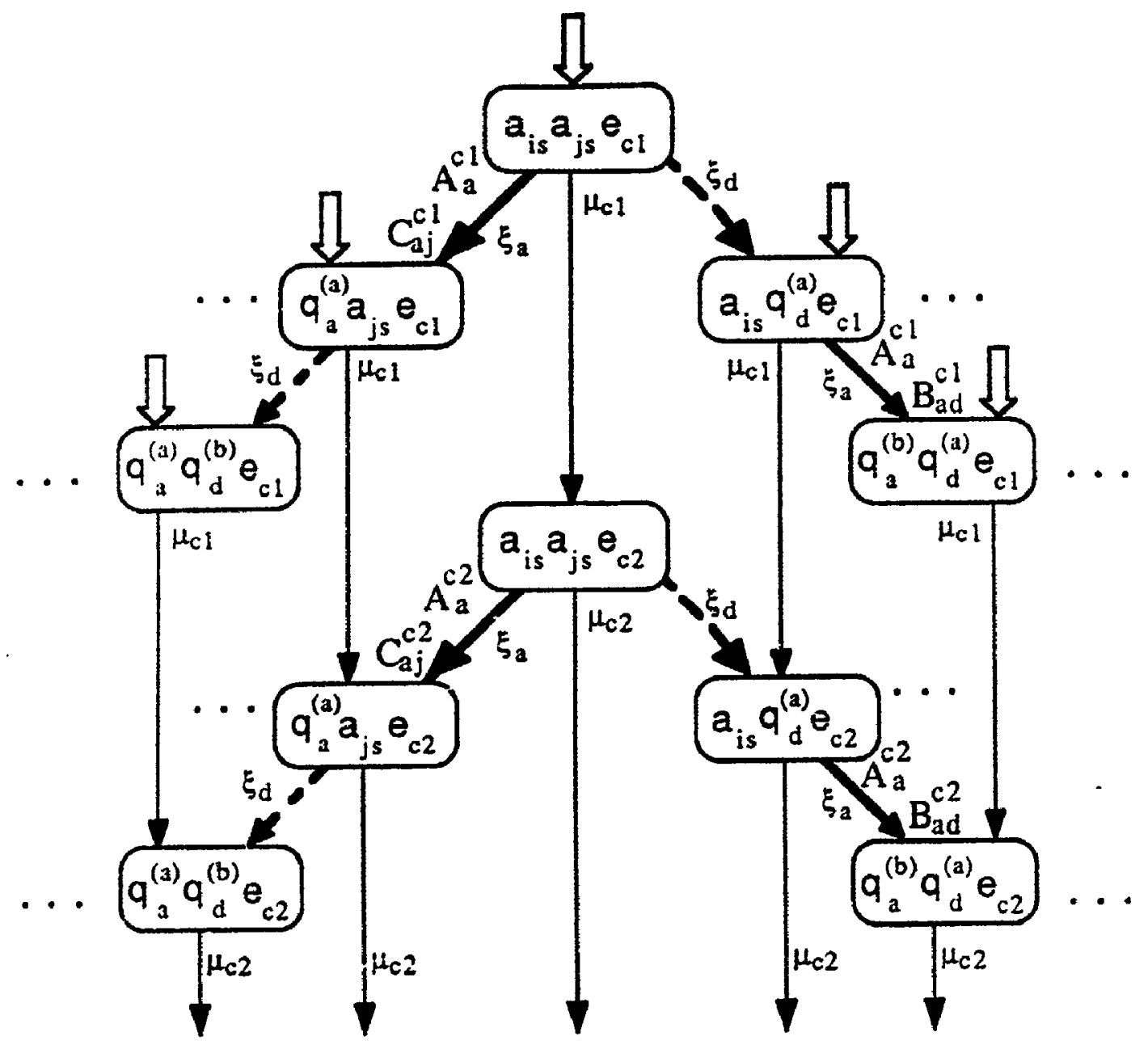

Figure 5.12: Group $\mathcal{G}_{k}$ of $\mathrm{MC}$ states aggregated for $D=\{i, j, s\}$, where $a \in \mathcal{R}_{i s}, d \in$ $\mathcal{R}_{j s}, c \in \mathcal{R}_{k s}$, and $c$ from $T_{k} k \neq i, j$ is in service

From (5.16), (5.23), and by using the definition for $A_{a}^{c 1}$ as ratio of flows we obtain:

$$
C_{a j}^{c 2}=\frac{\mu_{c 1}}{\mu_{c 2}+\lambda_{i s}+\lambda_{j s}} A_{a}^{c 1} \quad \text { for } a \in \mathcal{R}_{i s}, \quad c \in \mathcal{R}_{j s}
$$

Case 3: The server executes a request $c$ from a task $T_{k}$ that is different from $T_{i}$ and $T_{j}$. Figure 5.12 represents the group $\mathcal{G}_{c s}$ aggregated for $\{i, j, s\}$, where $a, \in \mathcal{R}_{i s}, d \in \mathcal{R}_{j s}$, and $c \in \mathcal{R}_{k s}$.

A request $a$ from $T_{i}$ may arrive to the server and find $d$ from $T_{j}$ already in queue in both phases of service for $c$. Thus both $B_{a d}^{c 1}$ and $B_{a d}^{c 2}$ are non-null. 
From the definitions of the arrival probabilities we have:

$$
A_{a}^{c p}=C_{a j}^{c p}+\sum_{b \in R, a} B_{a b}^{c p}
$$

We will use relation 5.25 to compute $\sum_{b \in \mathcal{R}_{j 8}} B_{a b}^{c p}$ as the difference between the other two probabilities. The components $B_{a d}^{c p}$ will be then approximated as follows:

$$
B_{a d}^{c p}=\pi_{d}\left(A_{a}^{c p}-C_{a j}^{c p}\right)
$$

$A_{a}^{c 1}$ and $A_{a}^{c 2}$ are known from the relations (5.8) and (5.12) applied to the aggregated MC where $T_{i}$ and $T_{k}$ are the designated clients.

The probability $C_{a j}^{c 1}$ is determinated approximately as in the single-class case from chapter 4, by making the assumption that the arrival of requests from $T_{i}$ and $T_{j}$ are independrut for the period when $T$, is serving $c$ in the first phase. This means that we can write:

$$
\frac{\mathcal{P}\left(a_{i s} a_{j s} e_{c 1}\right)}{\mathcal{P}\left(a_{j s} e_{c l}\right)}=\frac{\mathcal{P}\left(a_{i s} e_{c 1}\right)}{\mathcal{P}\left(e_{c 1}\right)}
$$

Similar with (5.16) we have:

$$
C_{a j}^{c 1}=\frac{\xi_{a} \mathcal{P}\left(a_{i s} a_{j s} e_{c 1}\right)}{F_{a}}
$$

By applying (5.7) to $\mathcal{F}\left(a_{i s} e_{c 1}\right)$ and $\mathcal{P}\left(a_{j s} e_{c 1}\right)$, and using then (5.27), (5.28) we obtain:

$$
C_{a j}^{c 1}=\frac{\mu_{c 1}}{F_{c}} \cdot \frac{\xi_{d}}{F_{d}} A_{a}^{c 1} A_{d}^{c 1}
$$

The probability $C_{a j}^{c 2}$ defined by (5.16) is determined now in function of $C_{a j}^{c 1}$ by using the balance equation (5.19):

$$
C_{a j}^{c 2}=\frac{\mu_{c 1}}{\mu_{c 2}+\lambda_{i s}+\lambda_{j s}} C_{a j}^{c 1} \quad \text { for } a \in \mathcal{R}_{i s}, \quad d \in \mathcal{R}_{j s}, \quad c \in \mathcal{R}_{j s}
$$

\subsection{Algorithm and experimental results}

\subsubsection{TDA/MVA(2) Algorithm}

The algorithm solves exactly simple SRVN models with 2 clients with multi-class recquests if the times spent by both clients in state $a_{i s}$ and by server in all states $e_{a p}$ are exponentially dist-ibuted, and the clients choose request classes randomly with constant prohatilicies $\pi_{a}, i \in \mathcal{I}_{s}$. The algorithm gives approximate results for more then two clients, due 
to equarions (5.29) and (5.26) that are approximate. All the other equations for the arrival probabilities are exact under the previous assumptions.

Equations (5.25), (5.26), (5.29), and (5.30) are used only when there are more than two clients. Equations (5.5), (5.6), (5.12), (5.8), (5.13), (5.18), (5.20), (5.22), (5.24), (5.25), (5.26), (5.29), and (5.30) represent a set of nonlinear equations that can be solved iteratively as follows:

Algorithm 2 TDA/MVA(2) algorithm for a simple client/server SRVN with clients with multi-class requests.

a) Initialize all $F_{a}, B_{a d}$ with some feasible values;

b) Compute new values for the arrival probabilities $A_{a}^{b 2}$ (eq. 5.6), $A_{a}^{c 1}$ (eq. 5.12), $A_{a}^{c 2}$ (eq. 5.8), $C_{a j}^{C p}$ (eqs. 5.20, 5.24, 5.29, 5.30), $B_{a d}$ (eqs. 5.13, 5.18, 5.22, 5.25, 5.26); Update the arrival instant probabilities using an under-relaxation strategy, by applying only half of the change for each probability, i.e. prob $_{\text {new }}=0.5\left(\right.$ prob old + prob $\left._{\text {computed }}\right)$;

c) Determine new values for all queueing delays $w_{a s}$ (eq. 5.5), and throughputs $F_{\text {is }}$ (eq. 5.1), Fs (eq. 5.2);

d) Repeat steps b), c) until the total change in the arrival instant probabilities values is less than a given tolerance.

The number of equations for the arrival instant probabilities used in step (b) of the algorithm is higher than in the algorithm from the previous chapter, since it depends here on the number of request classes for every client, not only on the number of clients as in the single class case.

Let us denote by $m=\left|\mathcal{I}_{s}\right|$ the total number of request classes, and by $m_{i}=m-\left|\mathcal{R}_{i s}\right|$ the number of request classes excluding task $T_{i}$. The order of computational complexity of the algorithm is given by the order of the highest number of probability components computed in step (b). While the number of the most arrival probabilities components is of $O\left(\max \left(m_{i}, m_{j}\right)\right)$, the the number of components of type $B_{a d}^{c p}$ given by $(5.26)$ is of $\mathcal{O}\left(\max \left(m_{i}, m_{j}, m_{k}\right)\right)$ where $T_{i}, T_{j}$ and $T_{i}$ are three different clients with the smallest number of request classes. 


\subsubsection{Experimental results}

The accuracy of TDA/MVA(2) algorithm was investigated by applying it to about two hundred cases, and comparing the results with the exact GSPN solutions. There were four test suites MR2, MR3, MR4, and MR5 with two, three, four, and five clients, similar to the test suites CS2, CS3, CS4, and CS5 used in the previous chapter for the single-class case. Figure 5.1, 5.2, and 5.3 show the communication graphs for the test suites MR3, MR4, and MR5 respectively, indicating also the values of the mean number of RV requests from clients to the server. In order to keep a close similarity between the single-class and multi-class examples, the total number of RV requests per client and the mean service times are the same in both test cases. (The service time values are given in tables B.1, B.2, and B.3 respectively).

As expected, the results for the test suite MR2 are exact; they are not shown here. The results for the other test suites with more than two clients are approximate; they are compared with exact GSPN results.

The throughput results for MR3, MRA and MR5 suites are shown in tables B.7, B.S, and B.9 respectively. The new algorithm performs much better than the heuristic MVA algorithm (see section 3.1.1) applied to the same test suites. Table 5.1 shows comparatively the average errors in client task throughputs obtained with the heuristic MVA and the new algorithm for test suites MR3, MR4 and MR5.

Table 5.1: Comparison of mean throughput error obtained with the old and new approximate SRVN algorithms

\begin{tabular}{||c|rrr||}
\hline SRVN & \multicolumn{3}{|c||}{ Experimental test suites } \\
Method & MR3 & MR4 & MR5 \\
\hline Heuristic MVA & $\mathbf{6 . 3 7 \%}$ & $8.32 \%$ & $10.60 \%$ \\
TDA MVA & $0.38 \%$ & $0.47 \%$ & $0.63 \%$ \\
\hline
\end{tabular}

Table 5.2 shows the increase, in average, of the maximum error per case with system unbalance for test suite MR5. Comparing it to Table 4.3, that gives the same kind of results for the similar single-class tesi suite CS5, we see that the multi-class algorithm performs a little better for unbalanced syst ms than the single-class algorithm from chapter 4 . This is 
due to the fact that the negative impact of very unbalanced entries on result accuracy is slightly mitigated by class changing.

Table 5.2: Algorithm accuracy for suite MR5 with respect to model unbalance

\begin{tabular}{|c||c|c|c||}
\hline & \multicolumn{3}{c||}{ Unbalance factor $r=\sqrt{r_{c s} r_{s}}$} \\
& $1 \leq r<5$ & $5 \leq r<10$ & $10 \leq r<100$ \\
\hline Number of cases & 27 & 38 & 30 \\
Avrg. of Max_err/case & $0.77 \%$ & $0.94 \%$ & $1.60 \%$ \\
\hline
\end{tabular}

Experiments have shown that the feasible values chosen for initialization in the first step of the algorithm do not have any impact on the final results. As the iteration progresses, the intermediate values for throughputs and arrival instant probabilities are no guaranteed to remain all the time feasible. However, the experiments have shown that they do converge toward feasible values. Some cases of oscillatory convergence have been observed for test cases with saturated server. From experience, the convergence is obtained quickly (a few dozen iterations) for the cases where the server is not saturated, but the number of iterations grows when the server approaches the saturation, and the model is unbalanced. 


\section{Chapter 6}

\section{TDA-based Decomposition of Complex SRVN Models}

\subsection{Decomposition of SRVN models into client/server submodels}

This chapter considers complex SRVN models with any number of servers (accepting tasks), and with multi-layered service, such that top-level servers may make requests to low-level servers. A decomposition algorithm (called TDA/DEC) is proposed for these SRVN models by following the TDA-based approach presented in section 3.2.3. A model is decomposed into a set of interrelated client/server submodels, such as those studied in the previous two chapters. The solution for the whole system is obtained by solving the submodels iteratively, using for this the algorithms TDA/MVA(1) for clients with single-class requests, and TDA/MVA(2) for clients with multi-class requests, respectively.

The present chapter has a limited goal: firstly, to show that the SRVN analysis is not restricted to 2-level models, by suggesting an iteration framework, and showing that it gives reasonably good results; and secondly, to demonstrate the power of the basic TDA approach.

The TDA-based decomposition approach presented in section 3.2.3 follows four steps:

a) divide the model into solvable subrnodels;

b) establish how to compute the parameters of a submodel (i.e. rates for new aggregated active task states) from imported results and model parameters;

c) determine the solution algorithm for every submodel; 
d) solve iteratively the model, by using the results of the previous steps.

The SRVN model will be divided into client/server submodels (that can be solved by the algorithms proposed in the previous two chapters) according to the following the rules:

- There is a submodel for each non-reference task.

- A submodel contains one non-reference task playing the role of server, and all the other (reference and non-reference) tasks that send RV requests directly to it playing the role of clients.

- An arc in the communication graph representing a direct RV communication between a requester and an acceptor appears as such in just one submodel, where the acceptor is the server and the requester is a client; the mean waiting delay for this RV is obtained as a result of the submodel.

- A task may appear in more than one submodel, playing a different role in every of them, and thus being represented differently.

Figure 6.1 illustrates the decomposition of an SRVN model with two reference and three non-reference tasks into three submodels.

We establish now how to compute new values for the parameters of each submodel in function of the results of the other submodels, by following step $B$ of the TDA-based approach presented in section 3.2.3. First we identify for each task the three classes of states defined in section 3.2.3 : active (independent execution), external passive (interaction with other submodels), and internal passive (interactions inside the submodel). After this, we apply TDA-2 to each task, aggregating the active and external passive states and computing aggregated surrogate rates from model parameters and from results imported from other submodels. The application of step $B$ to SRVN tasks playing the role of client and server, respectively, in single-class and multi-class submodels is discussed next. We use the notation for SRVN tasks with multiple entries presented in section 3.1.

\section{Submodels with single-class clients}

Client representation. As shown in chapter 4, a client with single-class requests is sending $\mathrm{RV}$ requests to a single entry of the server, while the server has different entries for its different 


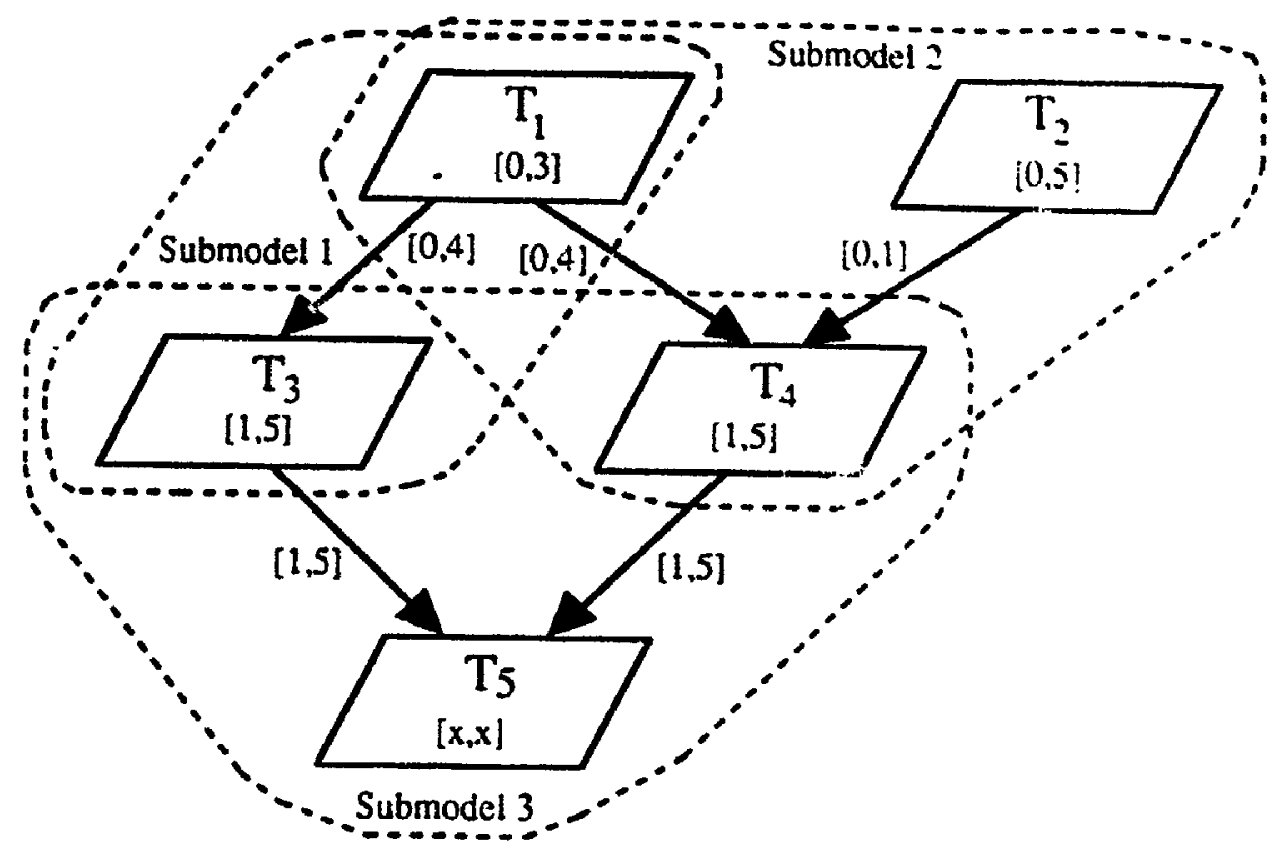

Figure 6.1: Decomposition of an SRVN model into submodels

clients. Let $T_{i}$ be an SRVN task that plays the role of a client in an SRVN submodel, sending singie-class RV requests to entry $g$ of task $T_{s}$. In the full model, $T_{t}$ can be either a reference or a non-reference task. Figure 6.2 illustrates the aggregation suffered by the state machine: of $T_{i}$ in both cases (as a reference task in (a) and a non-reference task in (b)) to produce the abstract representation of a client with single-class requests shown in $(c)$. The complete' state machine of $T_{i}$ (Figure 6.2.a or b, depending on the case) has the following classe's of task states:

- The active states of $T_{i}$ are the execution states $u_{i e p}$ for all entries $e \in E(i)$ and all phases $p$

- The external passive states of $T_{i}$ are the idle state $e_{i 0}$ (if there is one), and all the waiting states due to RV with tasks $T_{j}$ other than $T_{s}: q_{(i e p)(j d)}$ and $r_{(i e p)(j d)}$ for $e \in E(i)$, all $p$, $j \neq s$, and $d \in E(j)$

- The internal passive states of $T_{i}$ are all the waiting states due to RV with $T_{s}: q(1 ; p)(s g)$ and $r_{(i e p)(s g)}$ for $e \in E(i)$, and all $p$. 

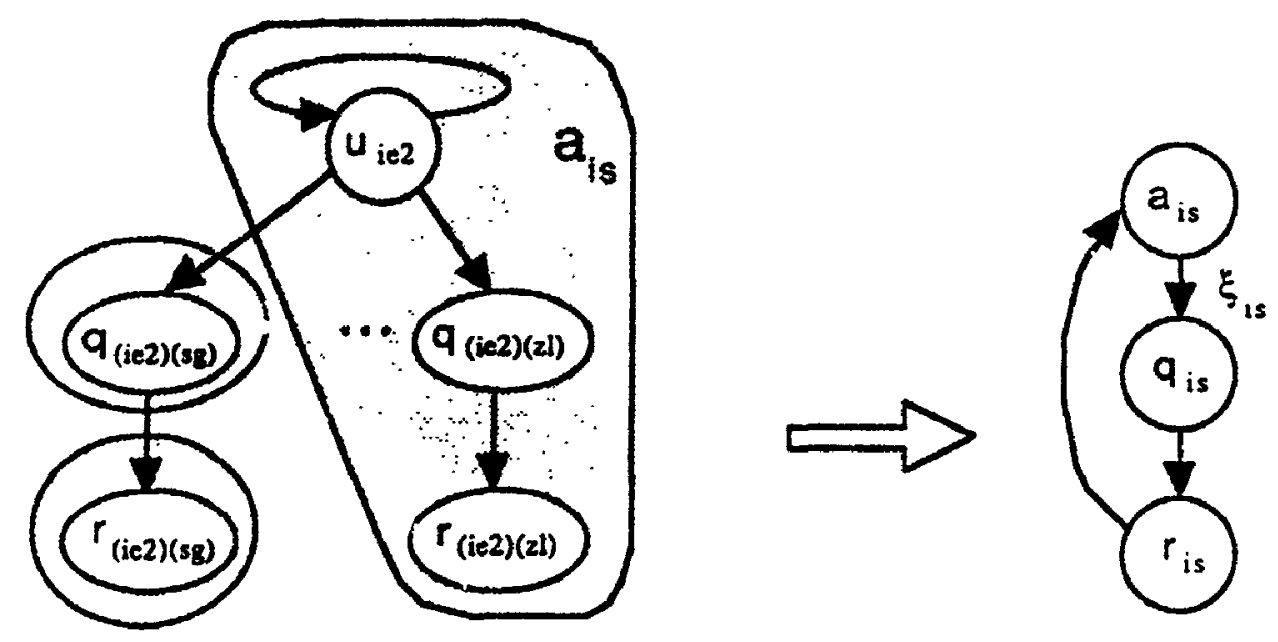

(a)

(c)

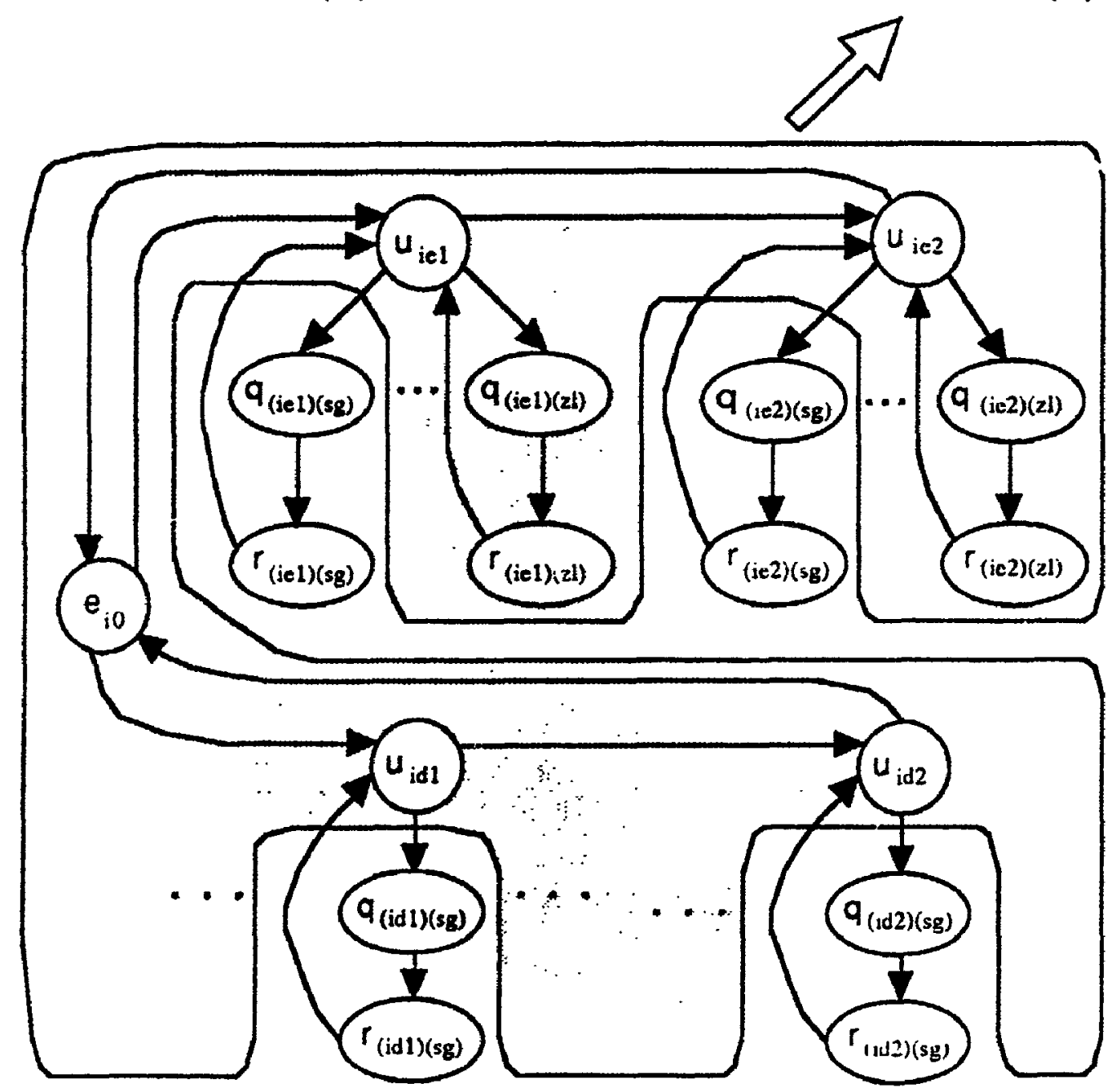

(b)

Figure 6.2: TDA-2 applied to (a) Reference task, and (b) Non-reference task to obtain (c) Abstract client with single-class requests 
The set of aggregated states for a client, shown in Figure 6.2.c has only three statess $\mathcal{S}_{\mathbf{v}}=$ $\left\{a_{i s}, q_{i,}, r_{i s}\right\}$ is obtained by TDA-2 as follows:

$a_{i s}=$ aggregated state grouping all the states where the client does not interact directly with the server (execution, idle, RV with other tasks than $T_{\bullet}$ );

$q_{i s}=U_{e} U_{p} q_{(i e p)(s g)}$, aggregated state where the client is waiting in queue at the server:

$r_{i s}=U_{e} U_{p} r_{(i e p)(s g)}$, aggregated state where the client is in RV with the server.

Since there is only one aggregated "active" state $a_{i s}$ (the other two being "passive"). ... have to determine only one aggregated surrogate rate $\xi_{i s}$ for the transition that leav's $a_{i s}$. The approach taken in this case is to determine $\xi_{\text {is }}$ directly from model parameters and imported results, instead of computing first the surrogate rates for all external passive states that are aggregated into $a_{i s}$. The results imported from other submodels for task $T_{i}$ are the throughputs $\varphi_{i e}$ for all entries $e \in E(i)$ of task $T_{i}$. As in the traffic equations 3.5. fir is expressed as a linear combination of reference throughputs. From entry throughputs wr determine the frequency $F_{i}$ of RV requests from $T_{i}$ to $T_{s}$, that in general can be written as:

$$
F_{i s}=\sum_{e \in E(i)} \varphi_{i c} \sum_{g \in E(s)} \sum_{p} y_{(i e p)(s g)}
$$

where $y_{(\text {iep })(e g)}$ is a model parameter defined in section 3.1. For the particular case of singleclass requests, the sum over $g$ can be dropped from relation 6.1 .

The rate $\xi_{i s}$ is then determined by applying relation 2.16 for aggregated rates as follows:

$$
\xi_{i s}=\frac{\sum_{e \in E(i)} \sum_{p} \eta_{i e p} \mathcal{P}\left(u_{i e p}\right)}{\mathcal{P}\left(a_{i s}\right)}
$$

where $\eta_{i e p}$ is the transition rate from state $u_{i e p}$ to $q_{(i e p)(s g)}$ in the non-aggregated state machine, determined from the initial parameters for $T_{i}$ as:

$$
\eta_{\text {iep }}=\frac{y_{(\text {iep })(s g)}}{s_{\text {iep }}}
$$

Expression 6.2 can be simplified by using additional information. The probability of the execution state $u_{\text {iep }}$ is:

$$
\mathcal{P}\left(u_{\text {iep }}\right)=\varphi_{\text {ie }} s_{\text {iep }}
$$


The probability of the aggregated state $a_{i,}$ is:

$$
\mathcal{P}\left(a_{i s}\right)=1-\left(\mathcal{P}\left(q_{i s}\right)+\mathcal{P}\left(r_{i s}\right)\right)=1-F_{i s}\left(w_{i s}+x_{s g 1}\right)
$$

where $w_{i s}$ and $x_{i g 1}$ are the queueing delay and the service time for a RV request from $T_{i}$ to $T_{s}$.

By replacing $6.3,6.4$ and 6.5 into 6.2 we obtain the aggregated rate $\xi_{i s}$ in function of imported results $\left(F_{i,}\right.$ and $\left.x_{s g 1}\right)$ and a previous result for the same submodel $\left(w_{i s}\right)$ :

$$
\xi_{i s}=\frac{1}{F_{i s}^{-1}-\left(w_{i s}+x_{s g 1}\right)}
$$

Server representation A non-reference task $T$, playing the role of server in a submodel has as external passive states all the waiting states for queueing and service due to nested RVs with other tasks:

$$
\left\{q_{(s g p)(j d)}, r_{(s g p)(j d)}\right\} \text { for } g \in E(s), \text { all } p, j \neq s
$$

Surrogate rates for these passive states are imported from other submodels, where $T$, plays the role of client and $T_{j}$ the role of server.

The set of aggregated states for a server $T_{\text {a }}$ in a submodel with single-class requests is

$$
\left\{e_{s 0}, e_{s 11}, \ldots, e_{s g p}, \ldots e_{s n 2}\right\}
$$

for $g=1, n ; p=1,2$, where:

$e_{s 0}=$ "server idle"

$e_{s g p}=$ "server executing entry $g$, phase $p$ " (active aggregated state including waiting for nested RVs)

An aggregated surrogate rate $\mu_{s g p}$ is determined for every aggregated state $e_{s g p}$ as:

$$
\mu_{s g p}=\frac{1}{x_{s g p}}
$$

where $x_{s g p}$ is the mean duration of the phase $p$ of task $s$, entry $g$, as defined in section 3.1.1, and determined by equation 3.1 , by using model parameters (execution times) and imported results (RV delays). This means that, at submodel level, we assume that the time spent in st ate $\epsilon_{s g p}$ is exponentially distributed with rate $\mu_{\text {sgp }}$. 


\section{Submodels with multi-class clients}

Client representation In the present research, a task $T_{i}$ is represented at the sulmoilel level as a client with multi-class requests when $T_{i}$ sends RV requests to more than one ent ry of the server $T_{\text {a. }}$ However, this representation neglects some characteristics of a RV rexplest. class. It was shown in section 5.1 that a request class is characterized not only by the destination entry at the server, but also by the the origin of the request (entry, pliase of the sending task, and path). These elements are not taken into consideration in the prese'lit. decomposition, since a path is "broken" into different submodels. Thus, all the request classes from a client requesting the same server entry are aggregated together, representing as separate only the classes with different destinations. This approximation, therefore, doe's not take into account the dependencies between different request classes arriving at a server. as for example the mutually exclusive requests discussed in section 5.1.

Figure 6.3 illustrates the aggregation by TDA-2 applied to a reference and to a $11 \mathrm{m-}$ reference task state machine, $(a)$ and $(b)$ respectively, in order to produce the representation (c) as a client with multi-class requests.

The client aggregated states include an active state $a_{i,}$ as in the single-class case just described, and a pair $q_{c}, r_{c}$ of aggregates states for every request class $c \in R_{i}$ sent by $T_{i}$ to Ts (see Figure 6.3.c).

In order to determine the aggregated surrogate rates $\xi_{c}$ for each $c \in R_{i s}$, we start by computing their sum $\lambda_{i s}=\sum_{c \in R_{i s}} \xi_{c}$, that is the total rate of leaving state $a_{i s}$.

As in the single-class case, we import for task $T_{i}$ the throughputs $\varphi_{i c}$ of all its entrics $e \in E(i)$. The total frequency $F_{i}$, of RV requests from $T$, to $T$, is determined as in 6.1 . The frequency $F_{c}$ of a class $c$ of requests sent by $T_{i}$ to entry $g$ of $T$, is given by:

$$
F_{c}=\sum_{e \in E(i)} \varphi_{i e} \sum_{p} y_{(i e p)(s g)}
$$

The mean probability that a RV request sent by $T_{i}$ to $T$, belongs to class $c$, denoted by $\pi_{r}$, is given by:

$$
\pi_{c}=\frac{F_{c}}{F_{i s}}
$$

The aggregated rate $\lambda_{i,}$ is obtained similarly to $\xi_{i s}$ from the single class case:

$$
\lambda_{i s}=\frac{\sum_{e \in E(i)} \sum_{p} \sum_{g \in E(s)} \eta_{(i e p)(\text { sg })} \mathcal{P}\left(u_{i e p}\right)}{\mathcal{P}\left(a_{i s}\right)}
$$



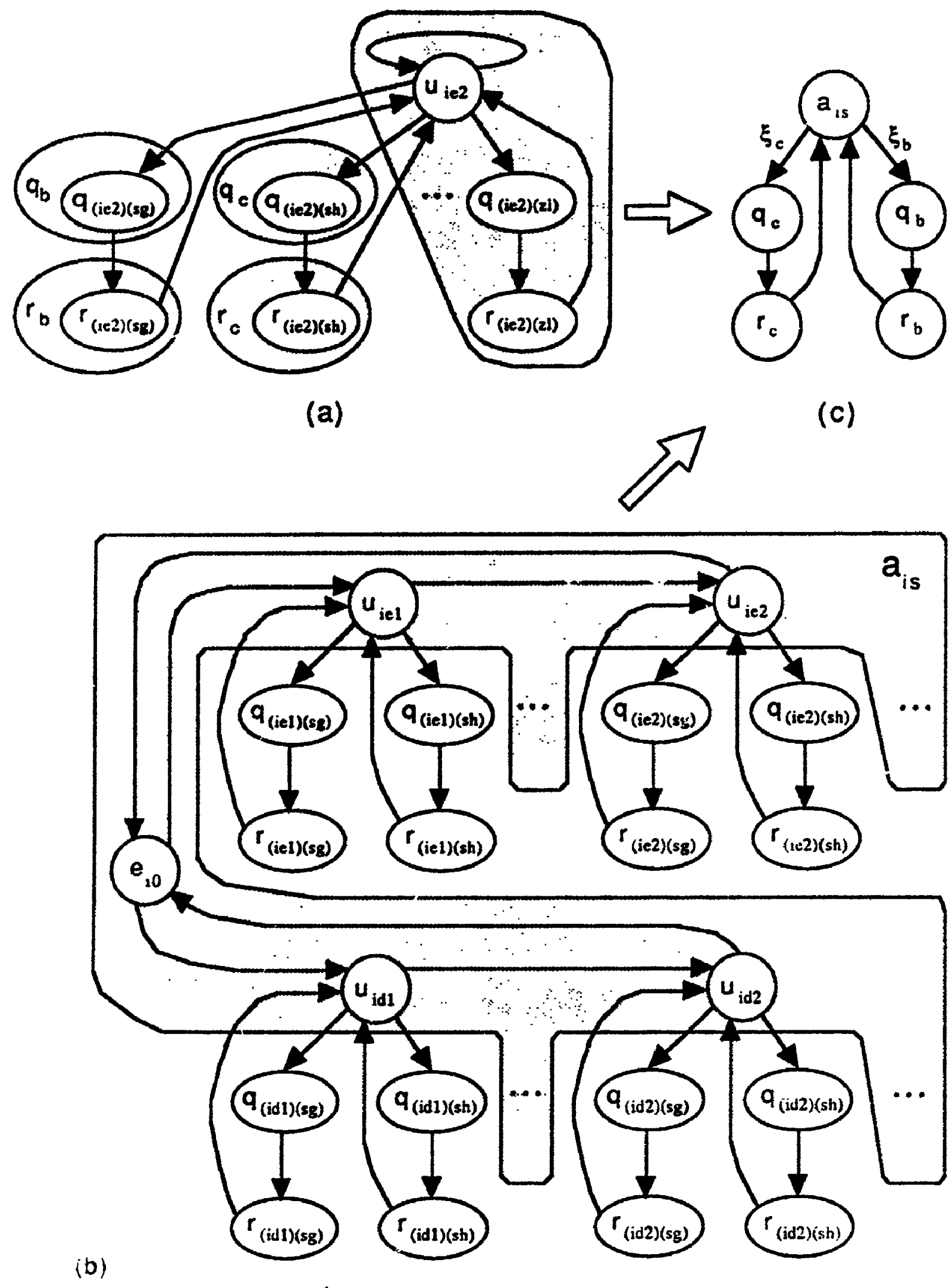

Figure 6.3: TDA-2 applied to (a) Reference task, and (b) Non-reference task to obtain (c) Abstract client with multi-class requests 
where $\eta_{(i e p)(g g)}$ is the transition rate from the execution state $u_{i e p}$ to the queueing state $q_{(\text {iep)(\&g) }}$ in the non-aggregated state machine. This is determined from the initial parameters of the model similarly to 6.3 :

$$
\eta_{(\text {iep })(\text { sg) }}=\frac{y_{(\text {iep })(s g)}}{s_{\text {iep }}}
$$

The probability of task $T_{i}$ being in the aggregated state $a_{i s}$ is determined as:

$$
\mathcal{P}\left(a_{i s}\right)=1-\sum_{c \in R_{i s}}\left(\mathcal{P}\left(q_{c}\right)+\mathcal{P}\left(r_{c}\right)\right)=1-\sum_{c \in R_{i s}} F_{c}\left(w_{c s}+x_{s g 1}\right)
$$

where $w_{c,}$ and $x_{\text {sgl }}$ are the queueing delay and the service time for a class $c$ request from $T_{i}$ to $T$.

By replacing $6.11,6.4,6.12$ and 6.9 into 6.10 we obtain:

$$
\lambda_{i s}=\frac{1}{F_{i s}^{-1}-\sum_{c \in R_{i s}} \pi_{c}\left(w_{c s}+x_{s g 1}\right)}
$$

The surrogate rates $\xi_{c}$ fo: each class $c$ are determined from $\lambda_{i s}$ as:

$$
\xi_{c}=\pi_{c} \lambda_{i}
$$

Server representation Similarly, for the case of multi-class requests, the representation of the server $T_{n}$, shown in Figure 5.5, contains two active aggregated states $e_{c 1}, e_{c 2}$ for earh request class $c$ arriving at $T_{s}$. An aggregated surrogate rate $\mu_{c p}$ is determined for every aggregated state $e_{c p}$ as:

$$
\mu_{c p}=\frac{1}{x_{s g p}}
$$

where $x_{\text {agp }}$ has the same meaning and computation as in 6.7 for the single-class case.

Step $C$ of ' 1 + TDA-based approach presented in section 3.2.3 is simply solved, since we use for submodel solution the approximate algorithms TDA/MVA(1) and TDA/MVA(2) developed in the previous two chapters.

Step $D$ of the TDA-based approach represents the iterative algorithm for solving an SRVN model by decomposition given in the following subsection.

\subsubsection{TDA/DEC Algorithm}

The following algorithm apply to complex SRVN models with any number of accepting tasks and of service layers. 
Aıgorithm 3 TDA/DEC decomposition algorithm for SRVN models.

a) Initialize queueing delays $w$ and reference throughputs $f_{r}$ with feasible values;

b) Apply the traffic equations 3.5 to determine entry throughputs $\varphi_{i}$ for all entries of nonreference tasks;

c) For each client/server a «bmodel

Compute model parameters:

for single-class case: $F_{i s}$ (eq. 6.1), $\xi_{\text {is }}$ (eq.6.6), $\mu_{\text {sgp }}$ (eq. 6.7)

for multi-class case: $F_{i s}$ (eq. 6.1), $F_{c}$ (eq. 6.8), $\xi_{c}$ (eq.6.14), $\mu_{c p}$ (eq. 6.15)

Solve submodel by:

Algorithm TDA/MVA(1) (chapter 4) for single-class case;

Algorithm TDA/MVA(2) (chapter 5) for multi-class case;

d) Compute new reference throughputs (eq. 3.2);

e) Repeat steps (b)-(e) until the total change in the arrival instant probabilities values is less than a given tolerance.

The algorithms TDA/MVA(1) and TDA/MVA(2) used to solve a submodel in step (c) are also iterative. In order to reduce the computation cost for the whole model, only one iteration of algorithm TDA/MVA(1) or TDA/MVA(2) is completed for each submodel at each step (c) repetition. The computational effort of step (c) is given by the the sum over all submodels of the effori reeded to complete one iteration step with algorithm TDA/MVA(1) or TDA/MVA(2), that depends in turn on the number of client request classes in the given submodel, as shown in sections 4.5 and 5.3 .1 , respectively. The overall complexity of the algotithm TDA/DEC is dependent not only on the computational complexity of each iteration, but also on the number of iterations required. It is quite difficult to predict the number of iterations necessary for the solution of a given SRVN model. Experiments have shown that the feasible values chosen for initialization in the first step of the algorithm do not have any impact on the final results. As the iteration progresses, the intermediate values for throughputs and arrival instant probabilities are no guarinteed to remain all the time 


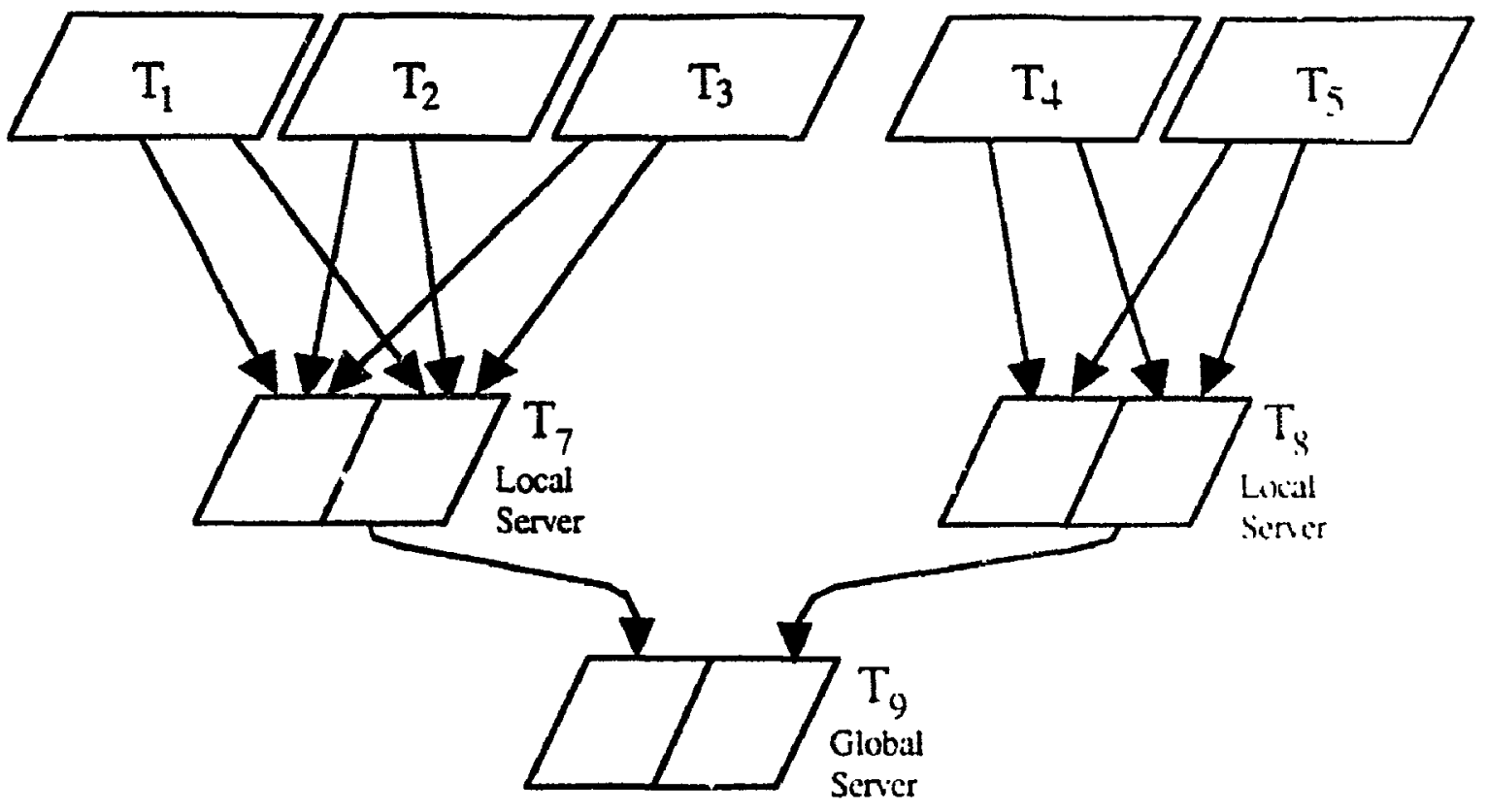

Figure 6.4: SRVN model for a hierarchical database: test suite IIDB1

feasible. However, the experiments have shown that they do converge toward feasible values. From experience it results that the convergence is obtained in a few dozen iterations for most. of the cases.

Experimental results obtained with algorithm TDA/DEC are reported in the following sections.

\subsection{Application: SRVN model for an hierarchical database}

In this section, algorithm TDA/DEC is applied to a multi-layered SRVN model representing a hierarchical database (HDB) system, that was introducer first as an example in section 1.1.4. Figures 6.4 and 6.5 show two test suite versions: the first, named HDB1, contains five users and three data servers, and the second, named HDB2, is obtained from the first by adding one more user. There are two local data servers, each with its own set of users, and a global data server receiving requests from the local servers only. Most of the user recpuests are served locally, and only a small fraction of requests (between $10 \%$ and $30 \%$ ) nered also the global server. (However, the ratio of requests in the last class is not small enmgh for 


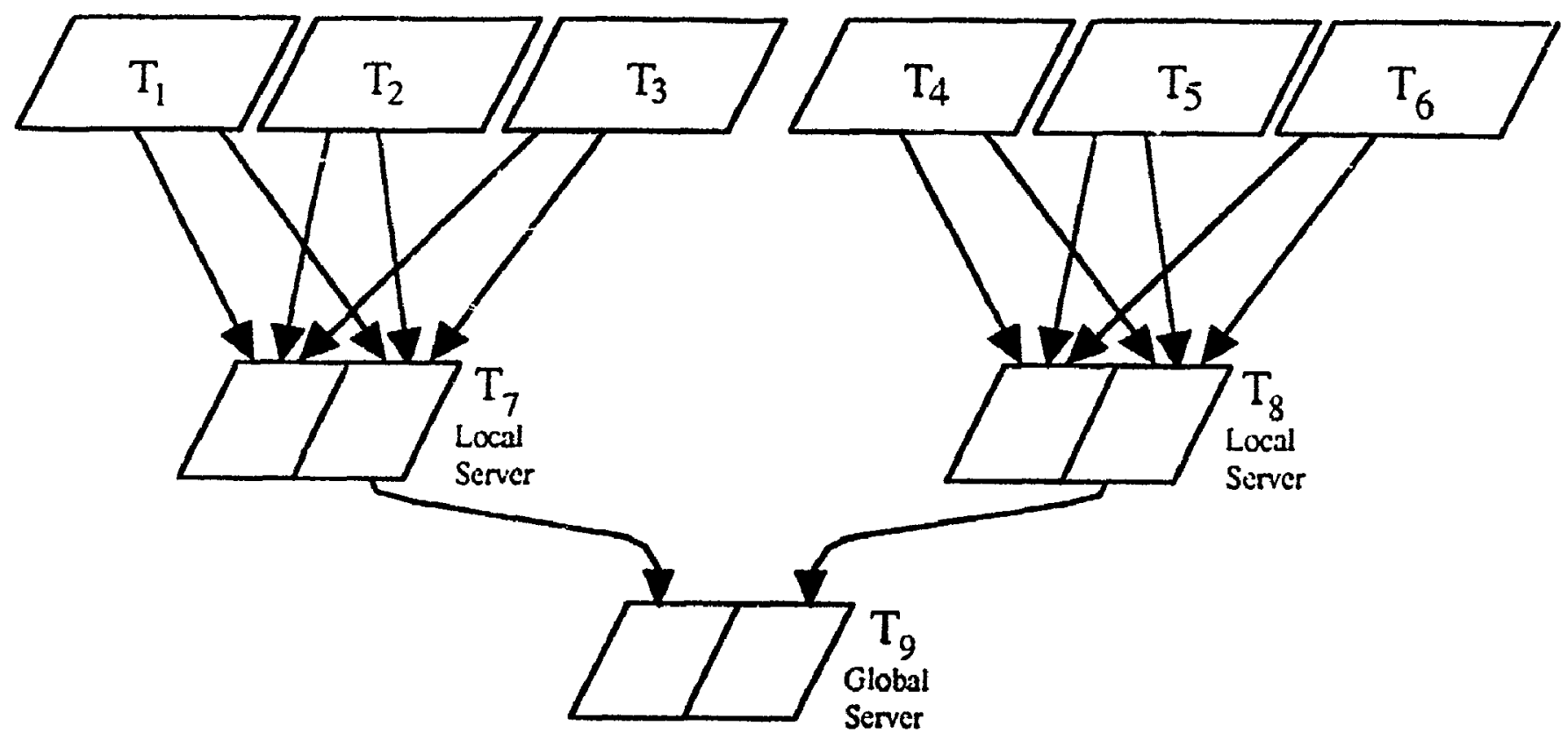

Figure 6.5: SRVN model for a hierarchical database: test suite HDB2

the the system to be considered nearly completely decomposable [Cou77]).

Each of the local servers has two entries in order to model the two classes of user requests: the first does not require any nested RVs, while the second sends RV requests to the global server. The global server also offers services with different workload for each of its clients.

Every server entry has an "in RV" phase (modelling the part of service in which the client is blocked waiting for a reply), and a "post RV" phase (modelling activities that can be executed in parallel with the client, such as writing files, sorting, or house-keeping).

As mentioned in section 1.1.4, a model with this kind of hierarchical service can be used to represent a rather wide range of "real life" systems:

- A software system implemented on a local area network, where several application processes (modelled as local servers) accept and serve requests from different users, sharing a database (or file) server (modelled as a global server). The application processes are running on different processors, and are provided with large $1 / O$ buffers in order to insure that a reasonable part of users' requests are served locally without accessing the global server. 
- A geographical distributed database, where most of the queries can be solved by a loral database server, while the rest need global information stored into a remote central server.

The SRVN model is able to capture the following features of the modelled system: mest od service offered by the hierarchy of servers, change of request classes by the users, and parallel execution between users and servers (second phases). As shown in section 3.1, SRVN does not represent directly the communication delays. The way to get around this mrob. lem is to include additional execution time in the first phase of service, corresponding 1 . communication delays for the RV request and the reply.

The purpose of this section is to assess experimentally the accuracy of the approximal.e results obtained with the decomposition algorithm TDA/DEC introduced in the previuns section, by comparing them with exact results obtained with GSPN models. Each of the $1 \mathrm{w}$, test suites HDB1 and HDB2 contains 30 cases divided into three groups $A, B$ and $C$, where $B$ and $C$ are obtained by modifying the "basic" group $A$. The execution times for servers, chosen to insure different degrees of unbalance between server entries and phase's are givan in table 6.1 for each of the 10 cases in a group. The execution times of the user tasks are the same for all cases: $s_{112}=10, s_{212}=15, s_{312}=12, s_{412}=5, s_{512}=8, s_{612}=10$ (the last) user is present only in suite HDB2).

Table 6.1: Execution Times of Servers T7, T8, T9 for a group from suite III)B1, HDIS2

\begin{tabular}{|c|c|c|c|c|c|c|c|c|c|c|c|c|}
\hline \multirow[t]{2}{*}{ Case } & \multicolumn{12}{|c|}{ DB Servers } \\
\hline & $s_{711}$ & $s_{712}$ & $s_{721}$ & $s_{722}$ & $s_{811}$ & $s_{812}$ & $s_{821}$ & $s_{822}$ & $s_{911}$ & $s_{912}$ & $s_{921}$ & $s_{922}$ \\
\hline$\overline{1}$ & 2.0 & 0.5 & 0.5 & 1.0 & 1.0 & 0.3 & 0.5 & 0.3 & 1.0 & 0.8 & 0.5 & 0.1 \\
\hline 2 & 1.0 & 0.2 & 0.2 & 0.5 & 1.0 & 0.3 & 0.5 & 0.3 & 1.0 & 0.8 & 0.5 & 0.4 \\
\hline 3 & 1.0 & 0.2 & 0.2 & 0.5 & 1.0 & 0.3 & 0.5 & 0.3 & 0.5 & 0.4 & 0.5 & 0.4 \\
\hline 4 & 1.0 & 0.2 & 0.2 & 0.5 & 1.0 & 0.3 & 0.5 & 0.3 & 0.5 & 0.4 & 0.2 & 0.2 \\
\hline 5 & 1.0 & 0.2 & 0.2 & 0.5 & 0.5 & 0.1 & 0.2 & 0.1 & 0.5 & 0.4 & 0.2 & 0.2 \\
\hline 6 & 2.0 & 0.5 & 0.5 & 1.0 & 1.0 & 0.3 & 0.2 & 0.1 & 0.5 & 0.4 & 0.2 & 0.2 \\
\hline 7 & 2.0 & 0.5 & 0.5 & 1.0 & 1.0 & 0.3 & 0.5 & 0.3 & 0.5 & 0.4 & 0.2 & 0.2 \\
\hline 8 & 4.0 & 1.0 & 1.0 & 2.0 & 1.0 & 0.3 & 0.5 & 0.3 & 1.0 & 0.8 & 0.5 & 0.4 \\
\hline 9 & 2.0 & 0.5 & 0.5 & 1.0 & 2.0 & 0.6 & 1.0 & 0.6 & 1.0 & 0.8 & 0.5 & 0.4 \\
\hline 10 & 2.0 & 0.5 & 0.5 & 1.0 & 1.0 & 0.3 & 0.5 & 0.3 & 2.0 & 1.6 & 1.0 & 0.8 \\
\hline
\end{tabular}


The mean number of $\mathrm{RV}$ requests is the same inside a group, but it is different from group to group, as shown in table 6.2. Compared to group $A$, the requests sent by the local servers to the global server in group $B$ are almost entirely moved from phase 1 to phase 2 , while the total number of requests for each server entry remains unchanged. This shortens the total server execution time in phase 1 (when the users are blocked) by reducing the number of nested RV/s, but keeps the same total workload for each server entry as in group $A$. In group $C$ compared to group $A$, the ratio of user requests that need service from the global server is increased, while the iotal number of user requests remains the same.

Table 6.2: Suite HDB1, HDB2 - Mean Number of RV requests (user T6 present only in HDB2)

\begin{tabular}{|c|cc|cc|c|c|}
\hline & \multicolumn{6}{|c|}{ From users to local server T7 } \\
Group & $y_{(112)(71)}$ & $y_{(112)(72)}$ & $y_{(212)(71)}$ & $y_{(212)(72)}$ & $y_{(312)(71)}$ & $y_{(312)(72)}$ \\
\hline A & 9.0 & 1.0 & 8.0 & 2.0 & 8.5 & 1.5 \\
B & 9.0 & 1.0 & 8.0 & 2.0 & 8.5 & 1.5 \\
C & 8.0 & 2.0 & 7.0 & 3.0 & 7.5 & 2.5 \\
\hline \multicolumn{6}{|c|}{ From users to local server T8 } \\
Group & $y_{(\mathbf{4 1 2 ) ( 8 1 )}}$ & $y_{(412)(82)}$ & $y_{(512)(81)}$ & $y_{(512)(82)}$ & $y_{(612)(81)}$ & $y_{(612)(82)}$ \\
\hline A & 8.5 & 1.5 & 9.0 & 1.0 & 8.0 & 2.0 \\
B & 8.5 & 1.5 & 9.0 & 1.0 & 8.0 & 2.0 \\
C & 7.5 & 2.5 & 8.0 & 2.0 & 7.0 & 3.0 \\
\hline
\end{tabular}

\begin{tabular}{|c|cc|cc|}
\hline & \multicolumn{4}{|c|}{ From local servers T, T8 to global server T9 } \\
Group & $y_{(721)(91)}$ & $y_{(722)(91)}$ & $y_{(821)(92)}$ & $y_{(822)(92)}$ \\
\hline A & 1.0 & 1.0 & 1.5 & 1.5 \\
B & 0.2 & 1.8 & 0.3 & 2.7 \\
C & 1.0 & 1.0 & 1.5 & 1.5 \\
\hline
\end{tabular}

Table 6.3 shows the average and the maximum relative error for reference throughputs and server utilization obtained with algorithm TDA/DEC for both test suites and all groups of cases.

The first observation is that the errors are higher than those reported in chapters 4 and 5 for client/server submodels. This is to be expected, since the algorithm TDA/DEC not only uses the approximate algorithms for client/server submodels, but also introduces supplementary approximation by decomposition and import of parameters from other submodels. However, the errors are still reasonable for the cases under consideration, as shown by table 6.3 . 
The maximum utilization errors are always due to the global server. The average utilization error is much lower than the maximum error, since it is computed over all the servers and includes also the small errors obtained for the local servers (that are close to saturation). It can be seen that there is no significant change in accuracy from suite HDB1 to suite HIDI::. The errors obtained for groups $B$ and $C$ are slightly higher than for the basic group $A(B$ has a bigger unbalance between phase 1 and phase 2 at the local servers, and $C$ has a higher competit:on for the global server).

Table 6.3: Relative throughput and utilization error for test suite HDis1, HIDI3:

\begin{tabular}{|cc|cc|cc|}
\hline & & \multicolumn{2}{|c|}{ Reference Throughput Error [\%] } & \multicolumn{2}{|c|}{ Server Utilization Error [\%] } \\
Case & Group & Average & Maximum & Average & Maximum \\
\hline \hline HDB1 & A & 2.93 & 5.80 & 1.17 & 5.08 \\
& B & 3.38 & 6.23 & 1.32 & 5.69 \\
C & 3.65 & 6.64 & 1.46 & 4.55 \\
\hline Total & 3.32 & 6.64 & 1.30 & 5.69 \\
\hline \hline HDB2 & A & 2.90 & 5.77 & 1.22 & 4.98 \\
& B & 3.45 & 6.19 & 1.40 & 5.64 \\
C & 3.40 & 6.63 & 1.33 & 4.41 \\
\hline Total & 3.25 & 6.63 & 1.32 & 5.64 \\
\hline
\end{tabular}

The accuracy of algorithm TDA/DEC for the cases considered here is better that that of the heuristic MVA algorithm . For example, the average throughput errors obtained with the heuristic MVA algorithm is of $8.06 \%$ for suite HDBI, and of $8.36 \%$ for suite III) 132 , compared to $3.32 \%$ and $3.25 \%$, respectively, as shown in table 6.3 .

The actual solution time experienced with the algorithm was of about 3.3 serconds in average for a case in test suite HDBI on a SPARC workstation. For the same model on the same workstation, the time needed to obtain the exact solution with the GroatSP.N package ([Chi87]) was about 700 times longer (98\% of the time being spent to build the. reachability graph, that has 8733 tangible and 47945 vanishing markings for HDI31). I'he biggest disadvantage of the Petri Nets approach, however, is the severe limitation in the sion. of models that can actually be solved, due to the state space explosion. The increase in the number of tangible states from HDB1 to HDB2 due to the addition of the sixth user is from 8733 to 29391 states. Furthermore, by adding to HDB2 one more user, we get a mordel that. 
was intractable, needing for the reachability graph more than the $37 \mathrm{M}$ of virtual memory that were available on the SPARC station used for experiment.

\subsection{Study of software bottleneck in multi-layered sys- tems}

This sections shows how an SRVN model can be used to identify performance "trouble spots" in software systems by studying the conditions in which a software bottleneck can appear. This phenomenon has been studied and modelled, for example, by Fontenot in [Fon89], under the name of "software congestion". It is possible to saturate a parallel system with no hardware resource being saturated due to a software server that is almost always busy, and thus throttles back the whole system. Due to the fact that the hardware resources are not saturated in this cases, the software bottleneck cannot be identified only by looking at hardware resources; but it is necessary to model and study the performance indices for soft ware components as well.

The software bottleneck can appear in any software systems where a software component (i.e. task in SRVN models) plays simultaneously the role of client and of server: the "busy" time as a server includes periods when the task itself plays the role of a client with respect to other system resources (hardware or software). Thus, even if the tas's is blocked, waiting to be served by the respective resources, it is nevertheless unable to accept another request from its input queue, being perceived as "busy" by its own clients. Therefore, the utilization of a software task is defined as the percentage of time when the task is "busy" as a server, including its own execution and the nested delays when it waits to be served by the resources required by itself.

An example of system with software bottleneck is the SRVN model shown in Figure 6.1, the possible bottleneck task being $\mathrm{T} 4$. To get the maximum parallelism possible, we assume that each task is running on its own processor. Figure 6.6 represents the utilizations of tasks $\mathrm{T} 4$ and $\mathrm{T} 5$, and that of processors $\mathrm{P} 4$ and $\mathrm{P5}$ on which the respective tasks are running, as functions of the execution time per phase at T5 (both phases being equal). (The plain lines represent the values obtained from a GSPN model, while the dotted lines represent approximate results obtained with the algorithm TDA/DEC). The utilization of task T5, 


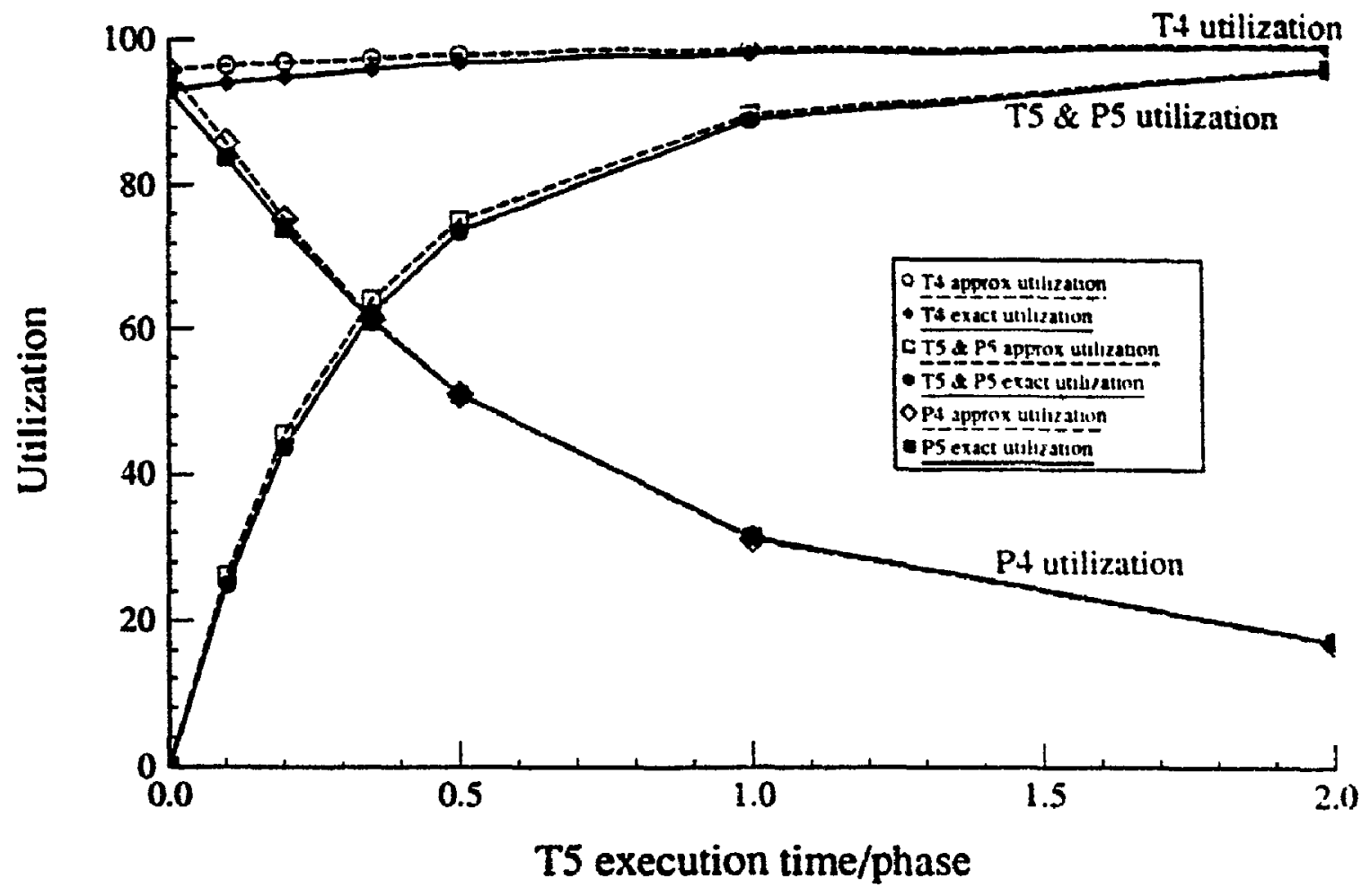

Figure 6.6: Exact and approximate utilization for tasks and processors

that does not make any nested RV requests, is identical at each moment with the utilization of processor P5. On the other hand, the utilization of task T4 contains three components, as shown in Figure 6.7: its own execution (identical to processor $\mathbf{P} 4$ utilization), in RV with ' 15 , and waiting in queue at T5. It can be seen from Figure 6.6 that the bottleneck in the system is task T4. This is especially important when neither of the processors is saturated, and the system is throttled back by $\mathrm{T} 4$. For example, in the case where $x=0.5$, T4 is the bottleneck, being busy over $98 \%$ of the time, while its own processor is utilized only about $51 \%$ and the processor P5 only $75 \%$ of the time. Understanding the behaviour of the system helps to decide what measures are necessary to improve the overall performance by alleviating the bottleneck. Separating the utilization of T4 in components, we realize that a general improvement can be obtained by changing the factors that affect any of these components. Thus, we can reduce either the execution time of T4, or that of T5 (by speeding up the processor, or by optimizing the code). The highest impact on performance is obtained for a change affecting the execution time on the processor with the highest utilization. In 'nur case, this is P4 for $x<0.35$ and P5 for $x>0.35$ (see Figure 6.6).

Anoiler interesting solution for this problem of software bottleneck does not reduce the 


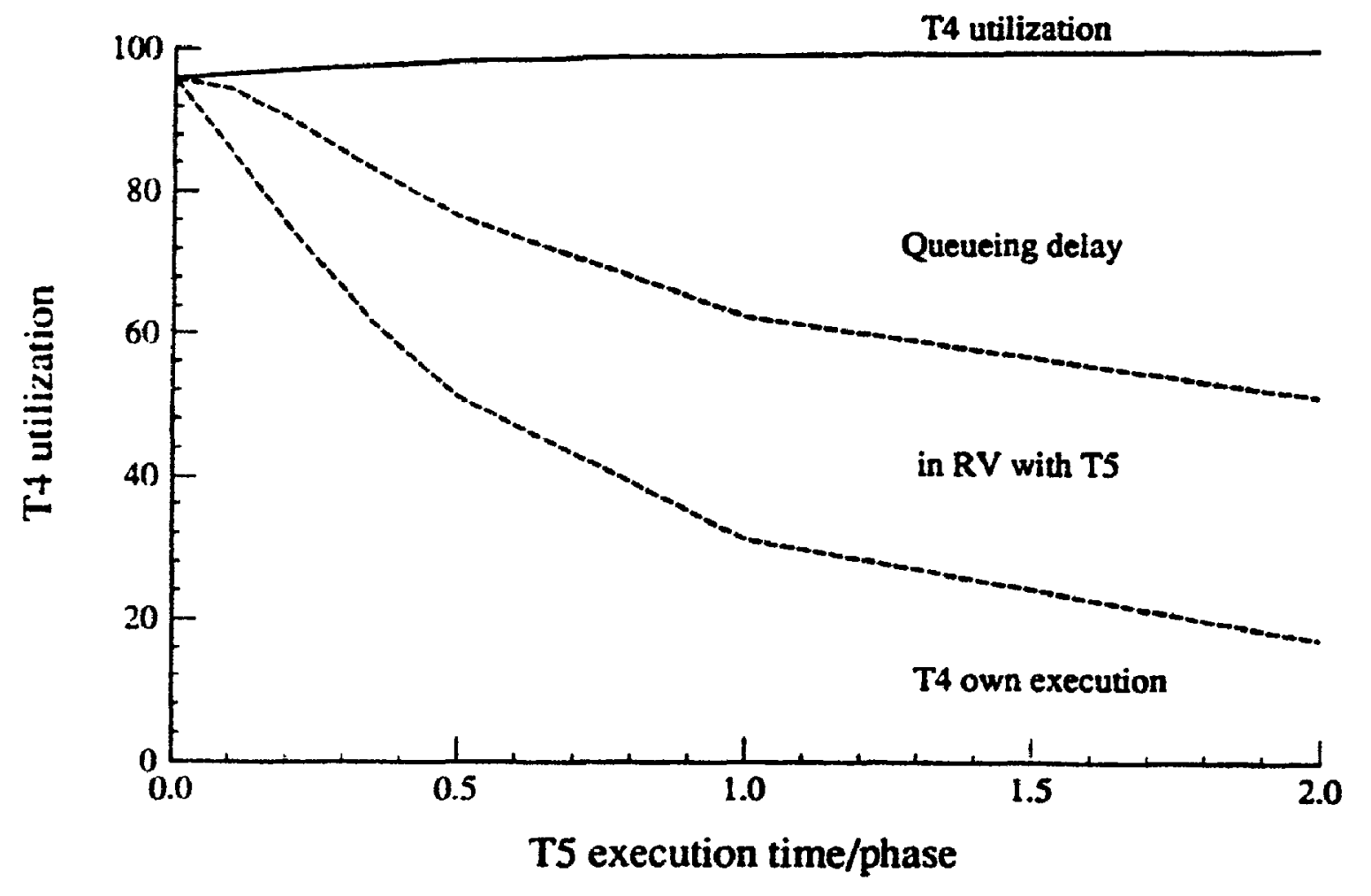

Figure 6.7: Utilization components for the bottleneck task T4

task execution times, but changes the tasking architecture by "cloning" the bottleneck server on the same processor. In this way, no hardware cost is involved, and the clones are still executed in mutual exclusion (that take care of the integrity of any common data structures). The improvement in overall performance is due to a better use of processor $\mathrm{P} 4$ capacity. Two configurations were considered: one with shared server clones as in Figure 6.8, another with dedicated clones as in Figure 6.9.

In order to solve this models with the current SRVN algorithm, we have to take into account the contention for the shared processor. Fortunately, as mentioned in section 1.1.4, the RV mechanism provides a natural way to take care of this problem, by representing a processor as a task with "in $\mathrm{RV}$ " phase only that is not making any nested RV requests. The execution time of the tasks allocated on a shared processor is transferred to the processor, such that an "execution slice" of the task between two RV requests will give the service time for the processor, and the number of "slices" will give the number of RV requests from task to procrssor. Since each task phase may have a different execution "slice" between 


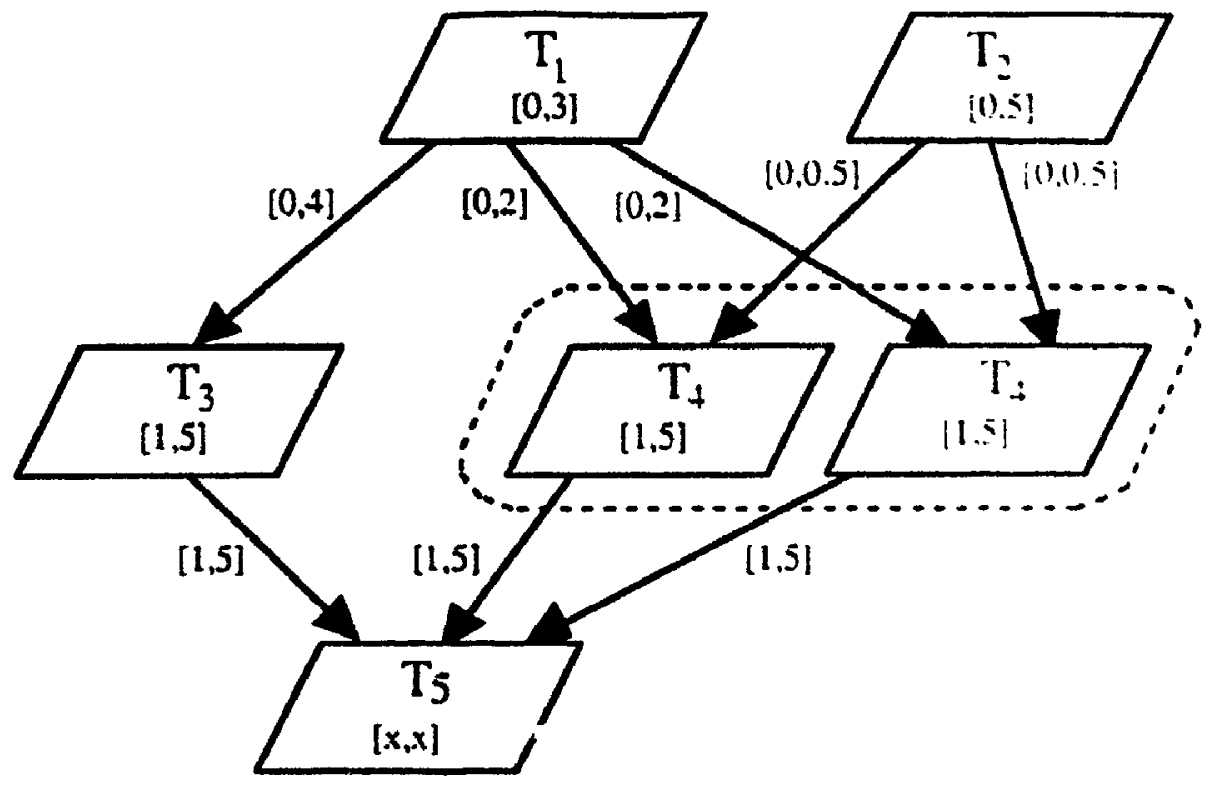

Figure 6.8: First software solution to alleviate the software bottleneck: shared server clones on the same processor

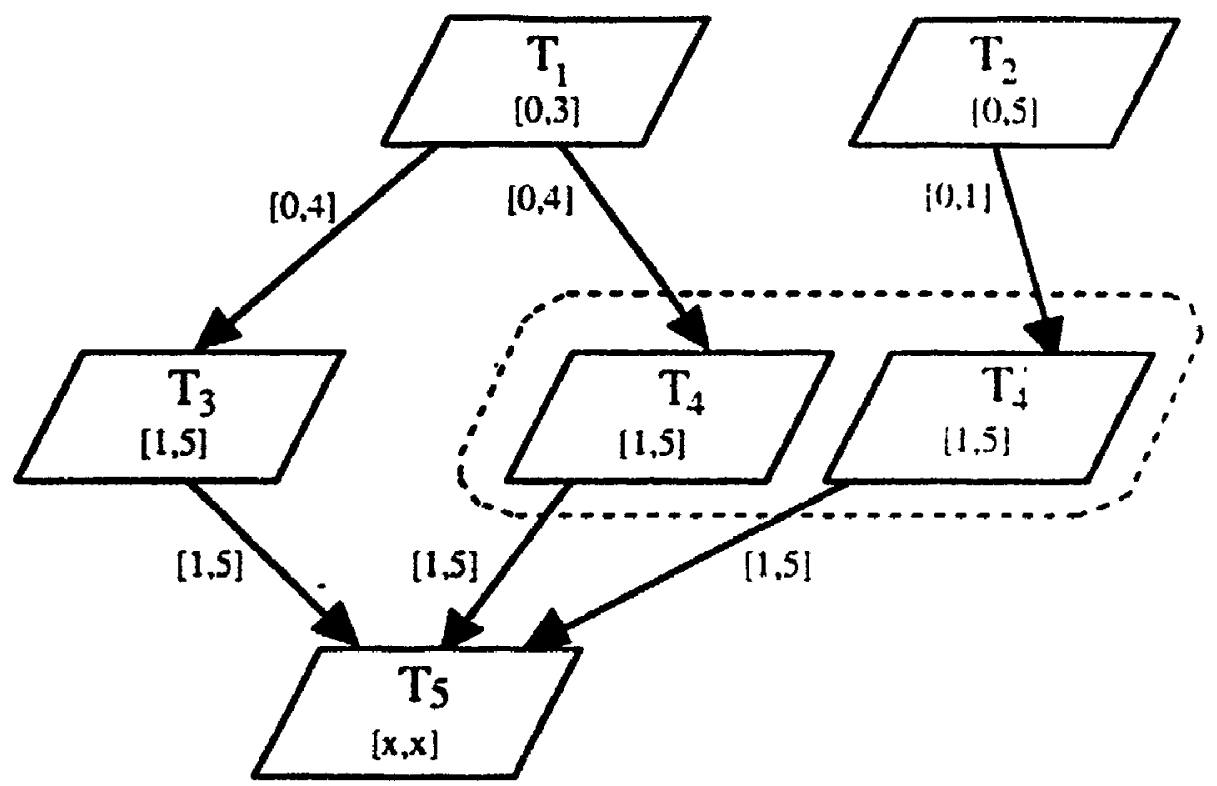

Figure 6.9: Second software solution to alleviate the software bottleneck: dedicated server clones on the same processor 


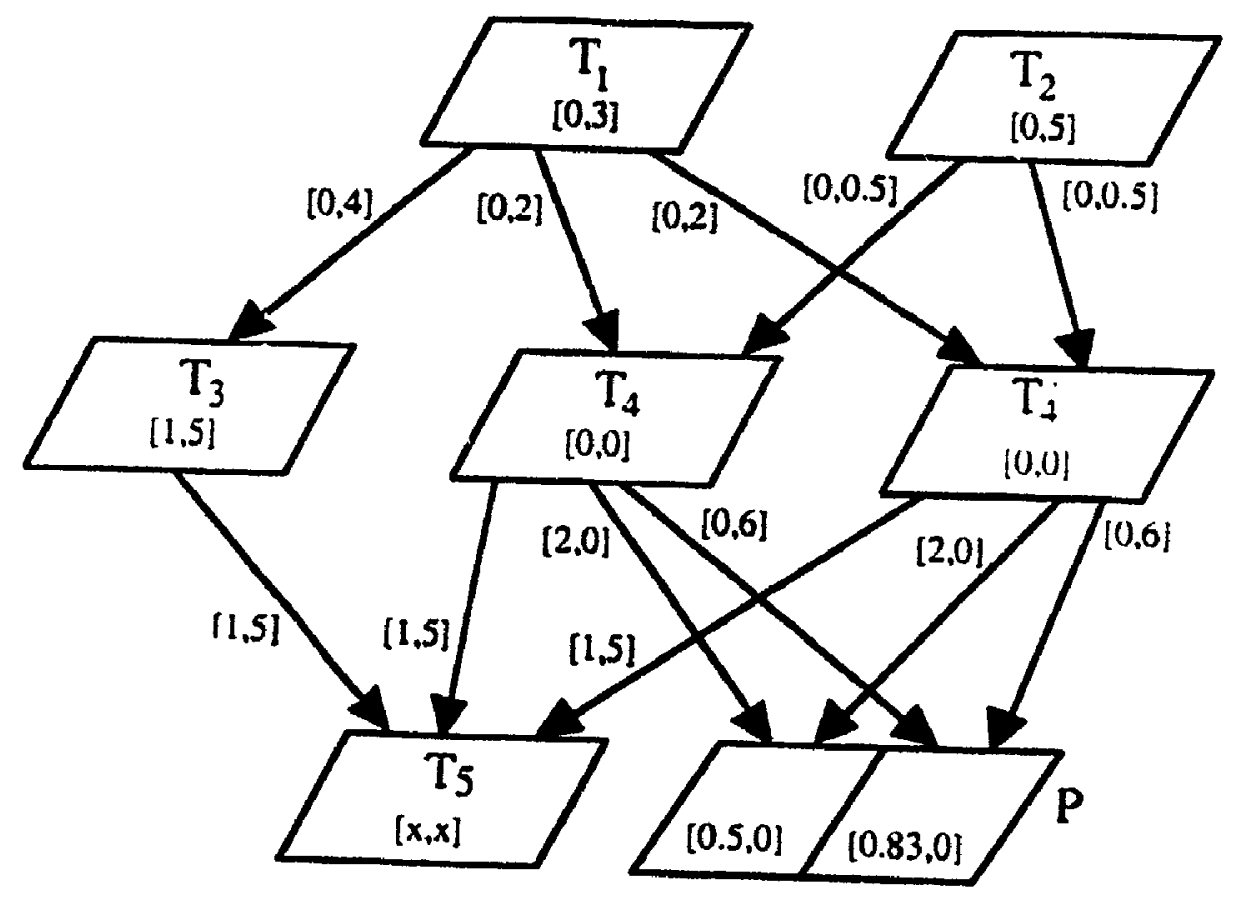

Figure 6.10: Transformation of the model from Figure 6.8 to represent the shared processor as an SRVN task

two RV requests, the SRVN task representing the processor will have several entries, one for each slice value. Figure 6.10 illustrates the transformation of the communication graph from Figure 6.8, by adding a new task for the shared processor. (A similar transformation was done for the model from Figure 6.9).

After making the necessary transformation and solving the models, we compare the results of the corresponding models without and with cloned servers. Table 6.4 shows the ratio of reference throughputs for the systems with cloned server over the throughputs for the initial system from Figure 6.1, obtained from SRVN results.

The gain in performance is more important for the case with dedicated clones. Also the improvement is higher for the middle cases, that are not strongly dominated by T4 or T5, and provide enough unused processor capacity to be exploited by the new server task.

We can see from this example that a software bottleneck is a complex phenomenon, involving more than one component, and, therefore, more difficult to understand and identify than a hardware bottleneck. While the latter can be identified directly from the model parameters, the former can be diagnosed only from the results of an appropriate performance 
Table 6.4: Improvement in reference throughput with respect to the system from Figure 6.1

\begin{tabular}{|c|cc|cc|}
\hline & \multicolumn{3}{|c|}{ Throughput improvement [\%] } \\
Shared clones & \multicolumn{2}{|c|}{ Dedicated clones } \\
\hline 0.2 & T1 & T2 & T1 & T2 \\
0.35 & 7.08 & 7.97 & 14.48 & 20.22 \\
0.5 & 4.66 & 15.88 & 16.76 & 24.42 \\
1.0 & -2.77 & 14.56 & 6.21 & 22.28 \\
\hline
\end{tabular}

model.

In conclusion, the SRVN modelling method, that captures relevant features of the soft witre systems with RV communication, helps the understanding of the system behaviour, the. identification of performance problems (such as software but leneck) and the asse'sime ont of the possible alternatives designed to improve the overall purformance. 


\section{Chapter 7}

\section{Conclusions}

The long term goals of this research are motivated by the general need for better analytical performance models for concurrent software system, able to capture relevant features that have a strong impact on the overall performance as, for example, those discussed in section 1.1.2. The problem of software modelling becomes even more important when we consider the idea that perfornance analysis should support the soltware development process throughout all its life cycle stages. The estimation of performance should begin early by assessing requirements and different design alternatives, while the widest range of options exists, and continue throughout the life cycle to implement systems that meet their performance sbjectives.

The short term goals of this research are related to the improvement of the solution methods for SRVN models of scftware with rendezvous communication, being motivated by two main factors:

- The need for a more rigorous theoretical foundation to replace the ad hoc collection of heuristic approximations used in the previous SRVN solutions;

- The search for SRVN solution methods with a better accuracy. The last requirement listed under long term goals points to approximate solution methods based on model decomposition. Given that the intended application is performance prediction in early software life cycle stages, when the software is not built yet, and the parameters for performance models are only approximate (from previous experience with similar systems or from prototyping), our accuracy goal is an average relative throughput error under $5 \%$, and a maximum error under $10 \%$. 
The goals have been achieved through the following contributions:

Theoretical foundation.

- Definition of Markov Chain "task-directed aggregation"(TDA) of two types: TDA-1 aggregates across tasks, so the identity of non-designated tasks disappears in the nuw state vector, while TDA-2 aggregates states within tasks.

- Study of TDA-1 and TDA-2 properties, by proving three lemmas on which arr hatsed the theorems stating the properties of the aggregated MC for simple client/server SRVN.

- Proposition of an approach based on TDA for the development of an iterative decomposition algorithm for a tasking system with synchronous communication, dealing specifically with the problem of obtaining submodel parameters from initial parameters and imported results from other submodels.

- Study of the Markov Chain for a simple client/server SRVN with a server and any number of clients $n$, giving its balance equations and showing that its size grows exponentially with the number of clients $n$. (Each client requests one entry of the server and gets a different service).

- Application of TDA to the MC of a simple client/server SRVN, proving two theorems that show that the size of the aggregated MCs with respect to the states of two and three designated tasks grows linearly with the number of clients $n$, and that the transition rate submatrices of the aggregated MCs have the same form for any number of clients $n$.

- Derivation of the equations for arrival instant probabilities from the balance equations of a set of aggregated MCs obtained by TDA for two cases of simple client/server SRVN, with clients with single and multi-class RV requests, respectively.

\section{SRVN solution algorithms}

- Algorithm TDA/MVA(1) for a simple client/server SRVN, where each client requests a single entry of the server (i.e. clients with single-class requests) Assessmentht 
of TDA/MVA(1) accuracy and convergence was done through experimental results, by applying it to about two hundred test cases and comparing the results with the exact GSPN solutions. The average relative throughput errors were under $1 \%$ and the maximum error under $4 \%$. The accuracy of TDA/MVA(1) is much better than the heuristic MVA algorithm, whose average error for the same test cases is just under $10 \%$. The convergence is usually obtained in a few dozen iterations, but the number of iterations grows when the server approaches the saturation, and the moael is unbalanced.

- Algorithm TDA/MVA(2) for a simple client/server SRVN with multi-class requests from each client to the server. Assessment of TDA/MVA(2) accuracy and convergence was also done through experimental results, by applying it to about two hundred test cases and comparing the results with the exact GSPN solutions. The average relative throughput errors were under $1 \%$ and the maximum error under $4 \%$. TDA/MVA(2) performs a little better for unbalanced systems than TDA/MVA(1), since the negative impact of very unbalanced entries on result accuracy is slightly mitigated by class changing. The convergence properties are similar to TDA/MVA(1).

- Iterative decomposition algorithm TDA/DEC for complex SRVN models with any number of servers and multi-layered service. The algorithm was applied to an SRVN model of an hierarchical database (60 test cases) obtaining average relative error for throughput under $4 \%$ and maximum iative error under $7 \%$. TDA/MVA(1) is more accurate than the heuristic MVA algurithm, whose average error for to the same test cases is just under $8.5 \%$. The convergence properties are similar io the previous two algorithms. TDA/DEC was also used to study a case of software bottleneck in multilayered system in order to demonstrate how SRVN helps the understanding of the system behaviour, the identification of performance problems, and the assessment of the possible alternatives designed to improve the overall performance.

\section{Limitations of the study}

Some of the most noticeable limitations of the present research are as follows:

- The study is limited to tasking systems with synchronous communication (rendezvous), excluding thus an important class of software systems with asynchronous communica- 
tion.

- The algorithms developed in the thesis cannot handle: non-exponential extrution times, other scheduling policies at the SRVN tasks than FIFO, open arrivals to the system.

- The accuracy studies presented in the thesis compare the approximate SRV $N$ results with exact Petri Net (GSPN) results. The size of the SRVN models used in te'st suiles: is therefore small, being limited by the Petri Net solution method. Further study of Ihe error behaviour in large size systems has to be done, by using for comparison simul.t iun results instead of Petri Net results. However, the simulation has two disadvantages: long execution times for simulation experiments, and the fact that the simulation results themselves are approximate.

\section{Directions for future research}

As a result of this work, several directions for future research were uncovered. They are' divided into two main categories: a) improving and extending the present SRVN solution methods, and b) increasing the modelling capabilities of SRVN.

a) Regarding the SRVN solution methods, the following directions for improvement and extension are open:

- a more thorough assessment of TDA/DEC accuracy, by applying it to a large variety of SRVN models of medium and large size.

- to take into account the dependency between classes of requests in the solution methor, by considering the mutually exclusive requests discussed in section 5.1 that are ignored in the present solution;

- to extend the algorithm to treat processors with priority scheduling (up till now, the processors are represented as SRVN tasks with "in RV" phase only, and the SRVN tasks have FIFO scheduling policy, as seen in section 6.3)

b) Some of the possible extensions of the SRVN modelling capabilities are: 
- to represent explicit communication delays and contention for bus (up till now, the communication delays have to be included explicitly in the service time of the "in RV" phase, and the contention for the communication medium is not accounted for)

- to extend the behaviour of the software tasks by introducing conditional accept of the requests arriving at different entries (in the current version, the requests are accepted indiscriminatedly in order of their arrival, one at a time). 


\section{Appendix A}

\section{Nondiagonal submatrices of $\mathbf{Q}^{\prime \prime}(n)$}

Here are given for completeness the nondiagonal submatrices $H^{\prime \prime}$ from the transition rals" matrix $Q^{\prime \prime}(n)$ (4.35) corresponding to an aggregated MC of a simple client/server SRVN, where the set of designated tasks is $D=\{i, j, s\}$.

$$
\mathbf{H}_{\mathbf{i j}}^{\prime \prime}=\left|\begin{array}{ccccccc}
0 & 0 & 0 & 0 & 0 & 0 & 0 \\
0 & 0 & 0 & 0 & 0 & 0 & 0 \\
0 & 0 & 0 & 0 & 0 & 0 & 0 \\
\pi_{41} \mu_{s i 2} & 0 & 0 & 0 & 0 & 0 & 0 \\
0 & 0 & 0 & 0 & 0 & 0 & 0 \\
0 & \pi_{62} \mu_{s i 2} & 0 & 0 & 0 & 0 & 0 \\
0 & 0 & 0 & 0 & 0 & 0 & 0
\end{array}\right| \quad \text { for } i, j \in D
$$

$$
\mathbf{H}_{\mathbf{i k}}^{\prime \prime}=\left|\begin{array}{cccccccccc}
0 & 0 & 0 & 0 & 0 & 0 & 0 & 0 & 0 & 0 \\
0 & 0 & 0 & 0 & 0 & 0 & 0 & 0 & 0 & 0 \\
\pi_{31} \mu_{s i 2} & 0 & 0 & 0 & 0 & 0 & 0 & 0 & 0 & 0 \\
0 & \pi_{42} \mu_{s i 2} & 0 & 0 & 0 & 0 & 0 & 0 & 0 & 0 \\
0 & 0 & \pi_{53} \mu_{s i 2} & 0 & 0 & 0 & 0 & 0 & 0 & 0 \\
0 & 0 & 0 & \pi_{64} \mu_{a i 2} & 0 & 0 & 0 & 0 & 0 & 0 \\
0 & 0 & 0 & 0 & \pi_{75} \mu_{s i 2} & 0 & 0 & 0 & 0 & 0
\end{array}\right| \text { for } i \in D, k \notin D
$$




$$
\mathbf{H}_{\mathbf{k i}}^{\prime \prime}=\left|\begin{array}{ccccccc}
0 & 0 & 0 & 0 & 0 & 0 & 0 \\
0 & 0 & 0 & 0 & 0 & 0 & 0 \\
0 & 0 & 0 & 0 & 0 & 0 & 0 \\
0 & 0 & 0 & 0 & 0 & 0 & 0 \\
0 & 0 & 0 & 0 & 0 & 0 & 0 \\
0 & 0 & 0 & 0 & 0 & 0 & 0 \\
0 & 0 & 0 & 0 & 0 & 0 & 0 \\
\pi_{81} \mu_{i k 2} & 0 & 0 & 0 & 0 & 0 & 0 \\
0 & 0 & 0 & 0 & 0 & 0 & 0 \\
0 & \pi_{10} 2 \mu_{i k 2} & 0 & 0 & 0 & 0 & 0
\end{array}\right| \text { for } i \in D, k \notin D
$$

$$
\mathbf{H}_{\mathbf{k l}}^{\prime \prime}=\left|\begin{array}{cccccccccc}
0 & 0 & 0 & 0 & 0 & 0 & 0 & 0 & 0 & 0 \\
0 & 0 & 0 & 0 & 0 & 0 & 0 & 0 & 0 & 0 \\
0 & 0 & 0 & 0 & 0 & 0 & 0 & 0 & 0 & 0 \\
0 & 0 & 0 & 0 & 0 & 0 & 0 & 0 & 0 & 0 \\
0 & 0 & 0 & 0 & 0 & 0 & 0 & 0 & 0 & 0 \\
\pi_{61} \mu_{s k 2} & 0 & 0 & 0 & 0 & 0 & 0 & 0 & 0 & 0 \\
0 & \pi 72 \mu_{s k 2} & 0 & 0 & 0 & 0 & 0 & 0 & 0 & 0 \\
0 & 0 & \pi_{83 \mu_{s k 2}} & 0 & 0 & 0 & 0 & 0 & 0 & 0 \\
0 & 0 & 0 & \pi_{94} \mu_{s k 2} & 0 & 0 & 0 & 0 & 0 & 0 \\
0 & 0 & 0 & 0 & \pi_{105} \mu_{s k 2} & 0 & 0 & 0 & 0 & 0
\end{array}\right| \text { for } k, l \notin D
$$

$$
\mathbf{H}_{\mathrm{i} 0}^{\prime \prime}=\left|\begin{array}{c}
0 \\
0 \\
\pi_{31} \mu_{s i 2} \\
0 \\
0 \\
0 \\
0
\end{array}\right| \text { for } i \in D \quad \mathbf{H}_{\mathbf{k} 0}^{\prime \prime}=\left|\begin{array}{c}
0 \\
0 \\
0 \\
0 \\
0 \\
\pi_{61} \mu_{s i 2} \\
0 \\
0 \\
0 \\
0
\end{array}\right| \text { for } k \notin D
$$

$$
\mathbf{H}_{\mathbf{0}}^{\prime \prime}=\left|\begin{array}{lllllll}
\xi_{i}, & 0 & 0 & 0 & 0 & 0 & 0
\end{array}\right| \quad \text { for } i \in D
$$

$$
\mathbf{H}_{\mathbf{0 k}}^{\prime \prime}=\left|\begin{array}{lllllllllll}
\xi_{i s} & 0 & 0 & 0 & 0 & 0 & 0 & 0 & 0 & 0
\end{array}\right| \text { for } k \notin D
$$




\section{Appendix B}

\section{Test suites for simple client/server SRVN}

Table B.1: Suite CS3 and MR3 parameters (3 users and onc servire)

\begin{tabular}{|c|c|c|c|c|c|c|c|c|c|}
\hline \multirow[t]{2}{*}{ Case } & \multicolumn{3}{|c|}{ Clients } & \multicolumn{6}{|c|}{ Server service times } \\
\hline & $s_{\mathrm{I}}$ & $s_{2}$ & $s_{3}$ & $s_{411}$ & $s_{412}$ & $s_{421}$ & $s_{422}$ & $s_{431}$ & $s_{4: 32}$ \\
\hline$T$ & 2.0 & 0.5 & 1.0 & 1.0 & 5.0 & 0.5 & 0.5 & 0.5 & 1.0 \\
\hline 2 & 1.0 & 0.5 & $1 / 3$ & 1.0 & 1.0 & 1.0 & 0.5 & 1.0 & $1 / 3$ \\
\hline 3 & 1.0 & 5.0 & 5.0 & 0.5 & 0.5 & 1.0 & 1.0 & 5.0 & 5.0 \\
\hline 4 & 1.0 & 10.0 & 0.5 & 1.0 & 1.0 & 1.0 & 10.0 & 0.5 & 0.5 \\
\hline 5 & 1.0 & 1.0 & 1.0 & 0.5 & 0.5 & 1.0 & 5.0 & 1.0 & 10.0 \\
\hline 6 & 1.0 & 5.0 & 0.5 & 1.0 & 1.0 & 5.0 & 5.0 & 0.5 & 0.5 \\
\hline 7 & 1.0 & 2.0 & 3.0 & 1.0 & 0.1 & 1.0 & 0.1 & 1.0 & 0.1 \\
\hline 8 & 1.0 & 2.0 & 3.0 & 0.1 & 1.0 & 0.1 & 1.0 & 0.1 & 1.0 \\
\hline 9 & 10.0 & 0.5 & 1.0 & 1.0 & 5.0 & 0.5 & 0.5 & 0.5 & 1.0 \\
\hline 10 & 100.0 & 0.5 & 1.0 & 1.0 & 5.0 & 0.5 & 0.5 & 0.5 & 1.0 \\
\hline 11 & 0.4 & 0.6 & 1.2 & 0.4 & 1.0 & 0.3 & 1.0 & 1.0 & 3.0 \\
\hline 12 & 1.0 & 2.0 & 0.5 & 1.0 & 0.2 & 2.0 & 0.5 & 1.0 & 1.0 \\
\hline 13 & 0.6 & 2.0 & 0.5 & 1.0 & 4.0 & 2.0 & 0.5 & 1.0 & 1.0 \\
\hline 14 & 0.8 & 1.0 & 0.5 & 2.0 & 4.0 & 1.0 & 0.1 & 1.0 & 1.0 \\
\hline 15 & 0.5 & 1.5 & 1.0 & 1.0 & 2.0 & 1.0 & 0.5 & 0.5 & 0.5 \\
\hline 16 & 2.0 & 0.5 & 0.8 & 5.0 & 5.0 & 0.5 & 0.5 & 0.5 & 1.0 \\
\hline 17 & 2.0 & 0.5 & 0.8 & 0.2 & 2.0 & 0.5 & 0.5 & 0.5 & 1.0 \\
\hline 18 & 2.0 & 0.5 & 0.8 & 0.2 & 2.0 & 2.0 & 3.0 & 0.5 & 1.0 \\
\hline 19 & 2.0 & 0.5 & 0.2 & 0.2 & 2.0 & 2.0 & $\mathbf{3 . 0}$ & 0.2 & 1.0 \\
\hline 20 & 2.0 & 0.5 & 0.5 & 0.2 & 2.0 & 2.0 & 3.0 & 0.5 & 0.5 \\
\hline 21 & 2.0 & 0.2 & 0.2 & 0.2 & 2.0 & 0.5 & 0.5 & 0.5 & 0.5 \\
\hline 22 & 2.0 & 0.2 & 1.0 & 0.2 & 2.0 & 0.5 & 0.5 & 0.2 & 0.2 \\
\hline 23 & 2.0 & 0.2 & 0.4 & 0.2 & 2.0 & 0.5 & 0.5 & 0.4 & 0.4 \\
\hline 24 & 2.0 & 0.2 & 1.2 & 0.5 & 2.0 & 0.5 & 0.5 & 0.4 & 0.4 \\
\hline 25 & 2.0 & 0.5 & 1.2 & 0.5 & 2.0 & 1.0 & 0.5 & 0.4 & 0.4 \\
\hline 26 & 2.0 & 0.5 & 1.2 & 0.5 & 2.0 & 1.0 & 2.0 & 0.1 & 0.4 \\
\hline 27 & 2.0 & 0.5 & 1.0 & 2.0 & 2.0 & 1.0 & 2.0 & 0.4 & 0.4 \\
\hline 28 & 1.0 & 0.5 & 1.0 & 0.5 & 2.0 & 0.5 & 2.0 & 1.0 & 1.0 \\
\hline 29 & 0.5 & 0.5 & 1.0 & 0.2 & 1.0 & 0.2 & 1.0 & 1.0 & 1.0 \\
\hline 30 & 0.5 & 0.5 & 1.0 & 1.0 & 0.2 & 2.0 & 0.2 & 1.0 & 0.2 \\
\hline 31 & 0.2 & 0.5 & 1.0 & 1.0 & 0.2 & 1.0 & 0.2 & 1.0 & 0.2 \\
\hline 32 & 2.0 & 0.5 & 1.0 & 1.0 & 0.2 & 1.0 & 0.2 & 1.0 & 0.2 \\
\hline 33 & 1.5 & 0.5 & 1.0 & 0.5 & 1.5 & 1.0 & 0.2 & 0.5 & 0.5 \\
\hline 34 & 4.0 & 0.5 & 1.0 & 1.0 & 5.0 & 2.0 & 1.0 & 0.5 & 0.5 \\
\hline 35 & 4.0 & 0.5 & 1.0 & 1.0 & 1.0 & 2.0 & 1.0 & 0.5 & 0.5 \\
\hline
\end{tabular}


Table B.2: Suite CS4 and MR4 parameters (4 users and one server)

\begin{tabular}{|c|c|c|c|c|c|c|c|c|c|c|c|c|}
\hline \multirow[t]{2}{*}{ Case } & \multicolumn{4}{|c|}{ Clients } & \multicolumn{8}{|c|}{ Server } \\
\hline & $s_{1}$ & $s_{2}$ & $s_{3}$ & $s_{4}$ & $s_{511}$ & $s_{512}$ & $s_{521}$ & $s_{522}$ & $s_{531}$ & $s_{532}$ & $s_{541}$ & \\
\hline 1 & 2.0 & $\overline{0.5}$ & 1.0 & 1.0 & 1.0 & 5.0 & 0.5 & $\overline{0.5}$ & 0.5 & 1.0 & 1.0 & 1.0 \\
\hline 2 & 2.0 & 0.5 & 1.0 & 0.5 & 1.0 & 5.0 & 0.5 & 0.5 & 0.5 & 1.0 & 1.0 & 0.1 \\
\hline 3 & 10.0 & 5.0 & 5.0 & 5.0 & 1.0 & 5.0 & 0.5 & 0.5 & 0.5 & 1.0 & 1.0 & 1.0 \\
\hline 4 & 2.0 & 2.0 & 2.0 & 2.0 & 0.2 & 1.0 & 0.2 & 1.0 & 0.2 & 1.0 & 0.2 & 1.0 \\
\hline ij & 2.0 & 2.0 & 2.0 & 0.5 & 0.2 & 1.0 & 0.2 & 1.0 & 0.2 & 1.0 & 0.2 & 0.2 \\
\hline 6 & 2.0 & 3.0 & 4.0 & 5.0 & 1.0 & 2.0 & 1.0 & 3.0 & 1.0 & 4.0 & 1.0 & 5.0 \\
\hline 7 & 2.0 & 2.0 & 1.0 & 1.0 & 1.0 & 0.5 & 0.5 & 1.0 & 0.5 & 1.0 & 1.0 & 1.0 \\
\hline 8 & 5.0 & 5.0 & 5.0 & 5.0 & 1.0 & 0.5 & 0.5 & 1.0 & 0.5 & 1.0 & 1.0 & 1.0 \\
\hline 9 & 2.0 & 0.5 & 1.0 & 0.1 & 1.0 & 1.0 & 0.5 & 0.5 & 0.5 & 1.0 & 0.1 & 1.0 \\
\hline 10 & 0.4 & 0.6 & 1.2 & 2.0 & 1.0 & 0.4 & 0.6 & 0.6 & 3.0 & 3.0 & 0.5 & 0.5 \\
\hline 11 & 0.4 & 0.6 & 1.2 & 2.0 & 0.4 & 1.0 & 0.3 & 1.0 & 1.0 & 3.0 & 1.0 & 2.0 \\
\hline 12 & 1.0 & 2.0 & 0.5 & 1.2 & 1.0 & 0.2 & 2.0 & 0.5 & 1.0 & 1.0 & 1.0 & 2.0 \\
\hline 13 & 0.6 & 2.0 & 0.5 & 1.2 & 1.0 & 4.0 & 2.0 & 0.5 & 1.0 & 1.0 & 1.0 & 2.0 \\
\hline 14 & 0.8 & 1.0 & 0.5 & 1.2 & 2.0 & 4.0 & 1.0 & 0.1 & 1.0 & 1.0 & 1.0 & 2.0 \\
\hline 15 & 0.5 & 1.5 & 1.0 & 1.2 & 1.0 & 2.0 & 1.0 & 0.5 & 0.5 & 0.5 & 1.0 & 0.5 \\
\hline 16 & 2.0 & 0.5 & 0.8 & 1.0 & 2.5 & 2.5 & 0.5 & 0.5 & 0.5 & 1.0 & 1.0 & 1.0 \\
\hline 17 & 2.0 & 0.5 & 0.8 & 1.0 & 0.2 & 2.0 & 0.5 & 0.5 & 0.5 & 1.0 & 1.0 & 1.0 \\
\hline 18 & 2.0 & 0.5 & 0.8 & 1.0 & 0.2 & 2.0 & 2.0 & 3.0 & 0.5 & 1.0 & 1.0 & 1.0 \\
\hline 19 & 2.0 & 0.5 & 0.2 & 1.0 & 0.2 & 2.0 & 2.0 & 3.0 & 0.2 & 1.0 & 1.0 & 0.5 \\
\hline 20 & 2.0 & 0.5 & 0.5 & 1.0 & 0.2 & 2.0 & 2.0 & 3.0 & 0.5 & 0.5 & 1.0 & 0.5 \\
\hline 21 & 2.0 & 0.2 & 0.2 & 1.0 & 0.2 & 2.0 & 0.5 & 0.5 & 0.5 & 0.5 & 1.0 & 0.5 \\
\hline 22 & 2.0 & 0.2 & 1.0 & 1.0 & 0.2 & 2.0 & 0.5 & 0.5 & 0.2 & 0.2 & 1.0 & 0.5 \\
\hline 23 & 2.0 & 0.2 & 0.4 & 1.0 & 0.2 & 2.0 & 0.5 & 0.5 & 0.4 & 0.4 & 1.0 & 0.5 \\
\hline 24 & 2.0 & 0.2 & 1.2 & 1.0 & 0.5 & 2.0 & 0.5 & 0.5 & 0.4 & 0.4 & 1.0 & 0.5 \\
\hline 25 & 2.0 & 0.5 & 1.2 & 1.0 & 0.5 & 2.0 & 1.0 & 0.5 & 0.4 & 0.4 & 1.0 & 0.5 \\
\hline 26 & 2.0 & 0.5 & 1.2 & 1.0 & 0.5 & 2.0 & 1.0 & 2.0 & 0.4 & 0.4 & 1.0 & 0.5 \\
\hline 27 & 2.0 & 0.5 & 1.0 & 1.0 & 2.0 & 2.0 & 1.0 & 2.0 & 0.4 & 0.4 & 1.0 & 0.5 \\
\hline 28 & 1.0 & 0.5 & 1.0 & 1.0 & 0.5 & 2.0 & 0.5 & 2.0 & 1.0 & 1.0 & 1.0 & 0.5 \\
\hline 29 & 0.5 & 0.5 & 1.0 & 1.0 & 0.2 & 1.0 & 0.2 & 1.0 & 1.0 & 1.0 & 1.0 & 0.5 \\
\hline 30 & 0.5 & 0.5 & 1.0 & $\mathbf{1 . 0}$ & 1.0 & 0.2 & 2.0 & 0.2 & 1.0 & 0.2 & 1.0 & 0.5 \\
\hline 31 & 0.2 & 0.5 & 1.0 & 1.0 & 1.0 & 0.2 & 1.0 & 0.2 & 1.0 & 0.2 & 1.0 & 0.5 \\
\hline 32 & 2.0 & 0.5 & 1.0 & 1.0 & 1.0 & 0.2 & 1.0 & 0.2 & 1.0 & 0.2 & 1.0 & 0.5 \\
\hline 33 & 1.5 & 0.5 & 1.0 & 1.0 & 0.5 & 1.5 & 1.0 & 0.2 & 0.5 & 0.5 & 1.0 & 0.5 \\
\hline 34 & 4.0 & 0.5 & 1.0 & 1.0 & 1.0 & 5.0 & 2.0 & 1.0 & 0.5 & 0.5 & 1.0 & 0.5 \\
\hline 35 & 4.0 & 0.5 & 1.0 & 1.0 & 1.0 & 1.0 & 2.0 & 1.0 & 0.5 & 0.5 & 1.0 & 0.5 \\
\hline
\end{tabular}


Table B.3: Suite CS5 and MR5 parameters ( 5 users and one server)

\begin{tabular}{|c|c|c|c|c|c|c|c|c|c|c|c|c|c|c|c|}
\hline \multirow[t]{2}{*}{ Case } & \multicolumn{5}{|c|}{ Clients } & \multicolumn{10}{|c|}{ Server } \\
\hline & $s_{1}$ & 82 & $s_{3}$ & $s_{4}$ & $s_{5}$ & $s_{611}$ & $s_{612}$ & $s_{621}$ & $s_{622}$ & $s_{631}$ & $s_{632}$ & $s_{641}$ & $s_{642}$ & $s_{651}$ & $s_{t i t}=$ \\
\hline$\overline{1}$ & 2.0 & 0.5 & 1.0 & 1.0 & 1.0 & 1.0 & 5.0 & $\overline{0.5}$ & 0.5 & 0.5 & 1.0 & 1.0 & 1.0 & 1.0 & 11 \\
\hline 2 & 2.0 & 0.5 & 1.0 & 0.5 & 0.5 & 1.0 & 5.0 & 0.5 & 0.5 & 0.5 & 1.0 & 1.0 & 0.2 & 1.0 & 07 \\
\hline 3 & 10.0 & 5.0 & 5.0 & $\mathbf{5 . 0}$ & 2.0 & 1.0 & 5.0 & 0.5 & 0.5 & 0.5 & 1.0 & 1.0 & 1.0 & 2.0 & 20 \\
\hline 4 & 1.0 & 2.0 & 3.0 & 4.0 & 5.0 & 1.0 & 5.0 & 0.5 & 0.5 & 0.5 & 1.0 & 1.0 & 1.0 & 2.0 & 211 \\
\hline 5 & 1.0 & 2.0 & 3.0 & 4.0 & 5.0 & 1.0 & 1.0 & 1.0 & 2.0 & 1.0 & 3.0 & 1.0 & 4.0 & 1.0 & 5.0 \\
\hline 6 & 1.0 & 2.0 & 3.0 & 4.0 & 5.0 & 1.0 & 5.0 & 1.0 & 4.0 & 1.0 & 3.0 & 1.0 & 2.0 & 1.0 & 1.0 \\
\hline 7 & 1.0 & 2.0 & 3.0 & 4.0 & 5.0 & 1.0 & 1.0 & 2.0 & 2.0 & 3.0 & 3.0 & 4.0 & 4.0 & 50 & 10 \\
\hline 8 & 1.0 & 2.0 & 3.0 & 4.0 & 5.0 & 1.0 & 1.0 & 2.0 & 1.0 & 3.0 & 1.0 & 4.0 & 1.0 & 5.0 & $1.1)$ \\
\hline 9 & 2.0 & 2.0 & 0.5 & 0.5 & 1.0 & 1.0 & 0.5 & 0.5 & 1.0 & 0.5 & 1.0 & 1.0 & 0.5 & 1.0 & 10 \\
\hline 10 & 5.0 & 5.0 & 5.0 & 5.0 & 5.0 & 1.0 & 0.5 & 0.5 & 1.0 & 0.5 & 1.0 & 1.0 & 0.5 & 1.0 & 111 \\
\hline 11 & 0.4 & 0.6 & 1.2 & 2.0 & 1.0 & 1.0 & 0.4 & 0.6 & 0.6 & 3.0 & 3.0 & 0.5 & 0.5 & 1.0 & 1.0 \\
\hline 12 & 0.4 & 0.6 & 1.2 & 2.0 & 1.0 & 0.4 & 1.0 & 0.3 & 1.0 & 1.0 & 3.0 & 1.0 & 2.0 & 1.0 & 2.0 \\
\hline 13 & 1.0 & 2.0 & 0.5 & 1.2 & 1.5 & 1.0 & 0.2 & 2.0 & 0.5 & 1.0 & 1.0 & 0.5 & 1.0 & 0.5 & 0.5 \\
\hline 14 & 0.6 & 2.0 & 0.5 & 1.2 & 1.5 & 1.0 & 4.0 & 2.0 & 0.5 & 1.0 & 1.0 & 0.5 & 1.0 & 0.5 & 0.5 \\
\hline 15 & 0.8 & 1.0 & 0.5 & 1.2 & 1.5 & 2.0 & 4.0 & 1.0 & 0.3 & 1.0 & 1.0 & 0.5 & 1.0 & 0.5 & 0.5 \\
\hline 16 & 0.5 & 1.5 & 1.0 & 1.2 & 1.5 & 2.0 & 1.0 & 1.0 & 0.5 & 1.0 & 0.5 & 0.5 & 0.5 & 1.0 & 0.5 \\
\hline 17 & 0.5 & 1.5 & 1.0 & 1.2 & 1. & 1.0 & 2.0 & 0. & 1.0 & 0.5 & 1.0 & 0.5 & 0.5 & 0.5 & 10 \\
\hline 18 & 2.0 & 2.0 & 2.0 & 0.5 & 0.5 & 0.2 & 1.0 & 0.2 & 1.0 & 0.2 & 1.0 & 0.2 & 0.2 & 0.2 & 02 \\
\hline 19 & 2.0 & 2.0 & 2.0 & 2.0 & 2.0 & 0.2 & 1.0 & 0.2 & 1.0 & 0.2 & 1.0 & 0.2 & 1.0 & 0.2 & 1.0 \\
\hline 20 & 1.0 & 1.0 & 1.0 & 1.0 & 1.0 & 1.0 & 1.0 & 1.0 & 1.0 & 1.0 & 1.0 & 1.0 & 1.0 & 1.0 & 1.0 \\
\hline 21 & 2.0 & 0.2 & 1.0 & 1.5 & 0.5 & 2.0 & 1.0 & 0.5 & 0.5 & 0.8 & 0.4 & 1.5 & 1.0 & 10 & 0.5 \\
\hline 22 & 2.0 & 0.2 & 1.0 & 1.5 & 0.5 & 0.5 & 0.5 & 0.5 & 0.5 & 0.8 & 0.4 & 1.5 & 1.0 & 1.0 & 0.5 \\
\hline 23 & 2.0 & 0.2 & 1.0 & 1.5 & 0.5 & 0.5 & 0.5 & 0.2 & 0.2 & 0.8 & 0.4 & 1.5 & 1.0 & 1.0 & (1) 5 \\
\hline 24 & 2.0 & 0.2 & 1.0 & 1.5 & 0.5 & 2.0 & 0.5 & 0.5 & 0.2 & 0.8 & 0.4 & 1.5 & 1.0 & 1.0 & 0.5 \\
\hline 25 & 2.0 & 0.2 & 1.0 & 1.5 & 0.5 & 1.0 & 0.2 & 1.0 & 0.5 & 0.8 & 0.4 & 1.5 & 1.0 & 10 & (0.5) \\
\hline 26 & 2.0 & 0.8 & 1.0 & 1.5 & 0.5 & 2.0 & 0.5 & 1.0 & 0.2 & 0.8 & 0.4 & 1.5 & 1.0 & 1.0 & 0.5 \\
\hline 27 & 2.0 & 0.8 & 1.0 & 1.5 & 0.5 & 0.5 & 2.0 & 0.2 & 1.0 & 0.4 & 0.8 & 1.0 & 1.5 & 0.5 & 1.0 \\
\hline 28 & 2.0 & 0.8 & 0.2 & 0.5 & 0.5 & 0.5 & 2.0 & 0.2 & 1.0 & 0.4 & 0.8 & 1.0 & 1.5) & 0.5 & 1.0 \\
\hline 29 & 2.0 & 0.8 & 0.2 & 0.5 & 0.5 & 0.5 & 0.5 & 0.2 & 0.2 & 0.4 & 0.4 & 1.0 & 1.0 & 0.5 & ر5 \\
\hline 30 & 2.0 & 0.8 & 0.2 & 0.5 & 0.5 & 1.0 & 0.5 & 0.5 & 0.2 & 0.8 & 0.4 & 2.0 & 1.0 & 10 & ر.5. \\
\hline 31 & 2.0 & 0.8 & 0.2 & 0.5 & 0.5 & 2.0 & 0.5 & 1.0 & 0.2 & 0.8 & 0.4 & 2.0 & 1.0 & 1.0 & 0.5 \\
\hline
\end{tabular}


CS5 parameters continued

\begin{tabular}{|c|c|c|c|c|c|c|c|c|c|c|c|c|c|c|c|}
\hline \multirow[t]{2}{*}{ Cise } & \multicolumn{5}{|c|}{ Clients } & \multicolumn{10}{|c|}{ Server } \\
\hline & $s_{1}$ & $s_{2}$ & $8_{3}$ & $s_{4}$ & 85 & 3611 & $s_{612}$ & $8_{621}$ & $s_{622}$ & $s_{631}$ & $s_{632}$ & $s_{641}$ & 8642 & $\delta_{651}$ & $s_{652}$ \\
\hline 32 & 2.0 & 1.5 & 1.0 & 0.5 & 1.2 & 2.0 & 2.0 & 1.5 & 1.5 & 1.0 & 1.0 & 0.5 & 0.5 & 1.2 & 1.2 \\
\hline 333 & 2.0 & 1.5 & 1.0 & 0.5 & 1.2 & 0.5 & 0.5 & 0.5 & 0.5 & 1.0 & 1.0 & 1.5 & 1.5 & 2.0 & 2.0 \\
\hline 34 & 2.0 & 1.5 & 1.0 & 0.5 & 1.2 & 0.2 & 0.5 & 0.2 & 0.5 & 0.5 & 1.0 & 0.8 & 1.5 & 1.0 & 2.0 \\
\hline 35 & 2.0 & 1.5 & 1.0 & 0.5 & 1.2 & 0.5 & 0.2 & 0.5 & 0.2 & 1.0 & 0.5 & 1.5 & 0.8 & 2.0 & 1.0 \\
\hline 36 & 10.0 & 5.0 & 5.0 & 5.0 & 2.0 & 1.0 & 10.0 & 0.5 & 0.5 & 0.5 & 1.0 & 1.0 & 1.0 & 2.0 & 2.0 \\
\hline 37 & 10.0 & 5.0 & 5.0 & 5.0 & 2.0 & 1.0 & 20.0 & 0.5 & 0.5 & 0.5 & 1.0 & 1.0 & 1.0 & 2.0 & 2.0 \\
\hline 38 & 10.0 & 5.0 & 5.0 & 5.0 & 2.0 & 1.0 & 1.0 & 0.5 & 0.5 & 0.5 & 1.0 & 1.0 & 1.0 & 2.0 & 2.0 \\
\hline 39 & 10.0 & 5.0 & 5.0 & 5.0 & 2.0 & 5.0 & 5.0 & 0.5 & 0.5 & 0.5 & 1.0 & 1.0 & 1.0 & 2.0 & 2.0 \\
\hline 40 & 10.0 & 5.0 & 5.0 & 5.0 & 2.0 & 10.0 & 5.0 & 0.5 & 0.5 & 0.5 & 1.0 & 1.0 & 1.0 & 2.0 & 2.0 \\
\hline 41 & 10.0 & 5.0 & 5.0 & 5.0 & 2.0 & 20.0 & 5.0 & 0.5 & 0.5 & 0.5 & 1.0 & 1.0 & 1.0 & 2.0 & 2.0 \\
\hline 42 & 1.0 & 1.0 & 1.0 & 1.0 & 1.0 & 1.0 & 3.0 & 1.0 & 3.0 & 1.0 & 3.0 & 1.0 & 3.0 & 1.0 & 3.0 \\
\hline 43 & 1.0 & 1.0 & 1.0 & 1.0 & 1.0 & 3.0 & 1.0 & 3.0 & 1.0 & 3.0 & 1.0 & 3.0 & 1.0 & 3.0 & 1.0 \\
\hline 44 & 1.0 & 1.0 & 1.0 & 1.0 & 1.0 & 3.0 & 3.0 & 3.0 & 3.0 & 3.0 & 3.0 & 3.0 & 3.0 & 3.0 & 3.0 \\
\hline 45 & 3.0 & 3.0 & 3.0 & 3.0 & 3.0 & 1.0 & 1.0 & 1.0 & 1.0 & 1.0 & 1.0 & 1.0 & 1.0 & 1.0 & 1.0 \\
\hline 46 & 3.0 & 3.0 & 3.0 & 3.0 & 3.0 & 1.0 & 3.0 & 1.0 & 3.0 & 1.0 & 3.0 & 1.0 & 3.0 & 1.0 & 3.0 \\
\hline $4 \bar{i}$ & 3.0 & 3.0 & 3.0 & 3.0 & 3.0 & 3.0 & 1.0 & 3.0 & 1.0 & 3.0 & 1.0 & 3.0 & 1.0 & 3.0 & 1.0 \\
\hline 18 & $\mathbf{3 . 0}$ & 3.0 & 3.0 & 3.0 & 3.0 & 3.0 & 3.0 & 3.0 & 3.0 & 3.0 & 3.0 & 3.0 & 3.0 & 3.0 & 3.0 \\
\hline 49 & 10.0 & 10.0 & 10.6 & 10.0 & 10.0 & 1.0 & 1.0 & 1.0 & 1.0 & 1.0 & 1.0 & 1.0 & 1.0 & 1.0 & 1.0 \\
\hline 50 & 10.0 & 10.0 & 10.0 & 10.0 & 10.0 & 3.0 & 1.0 & 3.0 & 1.0 & 3.0 & 1.0 & 3.0 & 1.0 & 3.0 & 1.0 \\
\hline 51 & 10.0 & 10.0 & 10.0 & 10.0 & 10.0 & 1.0 & 3.0 & 1.0 & 3.0 & 1.0 & 3.0 & 1.0 & 3.0 & 1.0 & 3.0 \\
\hline 52 & 100.0 & 100.0 & 100.0 & 100.0 & 100.0 & 1.0 & 1.0 & 1.0 & 1.0 & 1.0 & 1.0 & 1.0 & 1.0 & 1.0 & 1.0 \\
\hline 5.3 & 100.0 & 100.0 & 100.0 & 100.0 & 100.0 & 10.0 & 1.0 & 10.0 & 1.0 & 10.0 & 1.0 & 10.0 & 1.0 & 10.0 & 1.0 \\
\hline 54 & 100.0 & 100.0 & 100.0 & 100.0 & 100.0 & 1.0 & 10.0 & 1.0 & 10.0 & 1.0 & 10.0 & 1.0 & 10.0 & 1.0 & 10.0 \\
\hline 55 & 10.0 & 10.0 & 10.0 & 10.0 & 10.0 & 0.5 & 1.0 & 1.0 & 1.0 & 1.5 & 1.0 & .0 & 1.0 & 3.0 & 1.0 \\
\hline 56 & 10.0 & 10.0 & 10.0 & 10.0 & 10.0 & 0.5 & 0.5 & 1.0 & 1.0 & 1.5 & 1.5 & 2.0 & 2.0 & 3.0 & 3.0 \\
\hline 57 & 6.0 & 7.0 & 8.0 & 9.0 & 10.0 & 0.5 & 0.5 & 1.0 & 1.0 & 1.5 & 1.5 & 2.0 & 2.0 & 3.0 & 3.0 \\
\hline 58 & 10.0 & 9.0 & 8.0 & 7.0 & 6.0 & 0.5 & 0.5 & 1.0 & 1.0 & 1.5 & 1.5 & 2.0 & 2.0 & 3.0 & 3.0 \\
\hline 59 & 5.0 & 5.0 & 5.0 & 5.0 & 5.0 & 0.5 & 0.5 & 1.0 & 1.0 & 1.5 & 1.5 & 2.0 & 2.0 & 3.0 & 3.0 \\
\hline 60 & 3.0 & 3.5 & 4.0 & 4.5 & 5.0 & 0.5 & 0.5 & 1.0 & 1.0 & 1.5 & 1.5 & 2.0 & 2.0 & 3.0 & 3.0 \\
\hline 61 & 30.0 & 30.0 & 30.0 & 30.0 & 30.0 & 0.5 & 0.5 & 1.0 & 1.0 & 1.5 & 1.5 & 2.0 & 2.0 & 3.0 & 3.0 \\
\hline 62 & 18.0 & 21.0 & 24.0 & 27.0 & 30.0 & 0.5 & 0.5 & 1.0 & 1.0 & 1.5 & 1.5 & 2.0 & 2.0 & 3.0 & 3.0 \\
\hline
\end{tabular}


CS5 parameters continued

\begin{tabular}{|c|c|c|c|c|c|c|c|c|c|c|c|c|c|c|c|}
\hline \multirow[t]{2}{*}{ Case } & \multicolumn{5}{|c|}{ Clients } & \multicolumn{10}{|c|}{ Server } \\
\hline & $s_{1}$ & $\sigma_{2}$ & 83 & 84 & $s_{\mathbf{3}}$ & $s_{611}$ & $s_{612}$ & $s_{621}$ & $s_{622}$ & $s_{631}$ & $s_{632}$ & $s_{641}$ & $s_{\text {Fi2 }}$ & $s_{\text {sist }}$ & Stris: \\
\hline$\overline{63}$ & 2.8 & 4.2 & 7.0 & 9.0 & 10.0 & 0.5 & 0.8 & 3.2 & 1.6 & 1.5 & 3.5 & 2.7 & 1.0 & 3.8 & 1.0 \\
\hline 64 & 3.0 & 4.2 & 7.0 & 9.0 & 10.0 & 0.5 & 0.8 & 3.2 & 1.6 & 5.0 & 3.5 & 2.7 & 1.0 & 3.8 & 1.0 \\
\hline 65 & 5.0 & 4.2 & 7.0 & 9.0 & 10.0 & 2.0 & 0.8 & 3.2 & 1.6 & 10.0 & 10.0 & 2.7 & 1.0 & 3.8 & 4.0 \\
\hline 66 & 1.5 & 4.2 & 7.0 & 9.0 & 10.0 & 1.0 & 1.8 & 3.2 & 1.6 & 5.0 & 2.5 & 2.7 & 1.0 & 3.8 & 40 \\
\hline 67 & 1.5 & 4.2 & 7.0 & 10.0 & 20.0 & 1.0 & 1.8 & 3.2 & 1.6 & 5.0 & 2.5 & 2.7 & 1.0 & 3.8 & 111 \\
\hline 68 & 1.7 & 4.2 & 7.0 & 10.0 & 50.0 & 1.0 & 1.8 & 3.2 & 1.6 & 5.0 & 2.5 & 6.3 & 6.0 & 3.8 & 111 \\
\hline 69 & 1.7 & 4.2 & 7.0 & 10.0 & 50.0 & 2.0 & 1.8 & 3.2 & 1.6 & 5.0 & 2.5 & 6.3 & 6.0 & 20.8 & 10 \\
\hline 70 & 2.0 & 4.2 & 7.0 & 10.0 & 50.0 & 1.0 & 1.8 & 9.8 & 2.0 & 5.0 & 2.5 & 6.3 & 6.0 & 20.8 & 10 \\
\hline 71 & 2.5 & 4.2 & 7.0 & 10.0 & 49.0 & 1.0 & 1.8 & 9.8 & 2.0 & 5.0 & 2.5 & 6.3 & 6.0 & 15.0 & (i) 1 \\
\hline 72 & 2.5 & 4.2 & 7.0 & 20.0 & 49.0 & 1.0 & 1.8 & 9.8 & 2.0 & 5.0 & 2.5 & 6.3 & 6.0 & 15.0 & 150 \\
\hline 73 & 2.5 & 10.2 & 7.0 & 20.0 & 49.0 & 1.0 & 1.8 & 9.8 & 2.0 & 5.0 & 2.5 & 6.3 & 6.0 & 15.0 & $15,1)$ \\
\hline 74 & 1.0 & 10.2 & 7.0 & 10.0 & 45.0 & 0.5 & 0.8 & 3.2 & 1.6 & 5.0 & 2.5 & 2.7 & 1.0 & 3.8 & (1.1) \\
\hline 75 & 1.0 & 10.2 & 7.0 & 10.0 & 48.0 & 0.5 & 0.8 & 3.2 & 1.6 & 5.0 & 2.5 & 6. & 6.0 & $3 k$ & 1.0 \\
\hline 76 & 2.0 & 10.2 & 7.0 & 10.0 & 48.0 & 1.0 & 1.8 & 3.2 & 1.6 & 5.0 & 2.5 & 6 & 60 & $20 . x$ & 10 \\
\hline 77 & 2.0 & 10.2 & 7.0 & 10.0 & 50.0 & 1.0 & 1.8 & 9.8 & 2.0 & 5.0 & 2.5 & 6. & i. 0 & 20.8 & 10 \\
\hline 78 & 2.0 & 10.2 & 7.0 & 15.0 & 39.0 & 1.0 & 1.8 & 9.8 & 2.0 & 5.0 & 2.5 & 6. & 6.0 & 15.0 & 15.0 \\
\hline 79 & 2.0 & 6.2 & 7.0 & 10.0 & 29.0 & 1.0 & 1.8 & 3.2 & 1.6 & 5.0 & 2.5 & 6.3 & 6.0 & 20.8 & 10 \\
\hline 80 & 1.0 & 2.5 & 2.5 & 2.0 & 1.0 & 1.5 & 1.0 & 2.0 & 3.0 & 2.0 & 3.0 & 1.0 & 3.0 & 1.5 & 15 \\
\hline 81 & 1.0 & 2.5 & 2.5 & 2.0 & 1.0 & 3.0 & 2.0 & 5.0 & 6.0 & 4.0 & 6.0 & 2.0 & 5.5 & 2.9 & $2 ! 1$ \\
\hline 82 & 1.0 & 2.5 & 2.5 & 10.0 & 5.0 & 3.0 & 2.0 & 5.0 & 6.0 & 4.0 & 6.0 & 2.0 & 5.5 & 2.9 & 29 \\
\hline 83 & 1.0 & 2.5 & 2.5 & 20.0 & 10.0 & 3.0 & 2.0 & 5.0 & 6.0 & 4.0 & 6.0 & 2.0 & 5.5 & 2.9 & $2: 1$ \\
\hline 84 & 1.0 & 2.5 & 2.5 & 40.0 & 20.0 & 3.0 & 2.0 & 5.0 & 6.0 & 4.0 & 6.0 & 2.0 & 5.5 & 2.9 & $2 !$ \\
\hline 85 & 1.0 & 2.5 & 2.5 & 40.0 & 45.0 & $\mathbf{3 . 0}$ & 2.0 & 5.0 & 6.0 & 4.0 & 6.0 & 2.0 & 5.5 & 2.9 & 2.9 \\
\hline 86 & 1.0 & 2.5 & 2.5 & 100.0 & 80.0 & 3.0 & 2.0 & 5.0 & 6.0 & 4.0 & 6.0 & 2.0 & 5.5 & 2.9 & 2.9 \\
\hline 87 & 1.0 & 2.0 & 3.0 & 4.0 & 5.0 & 1.5 & 1.5 & 3.0 & 3.0 & 4.5 & 4.5 & 6.0 & 6.0 & 7.5 & 7.5 \\
\hline 88 & 1.0 & 2.0 & 3.0 & 4.0 & 5.0 & 1.5 & 1.0 & 3.0 & 2.0 & 4.5 & 3.0 & 6.0 & 4.0 & 7.5 & 5.0 \\
\hline 89 & 1.0 & 2.0 & 3.0 & 4.0 & 5.0 & 1.0 & 1.5 & 2.0 & 3.0 & 3.0 & 4.5 & 4.0 & 6.0 & 5.0 & 75 \\
\hline 90 & 1.0 & 2.0 & 5.0 & 8.0 & 10.0 & 1.5 & 1.5 & 3.0 & 3.0 & 10.0 & 10.0 & 16.0 & 16.0 & 20.0 & 20.0 \\
\hline 91 & 1.0 & 2.0 & 5.0 & 8.0 & 10.0 & 1.0 & 1.5 & 2.0 & 3.0 & 5.0 & 10.0 & 8.0 & 16.0 & 10.0 & 20.0 \\
\hline 92 & 1.0 & 20.0 & 50.0 & 80.0 & 100.0 & 50.0 & liv. 0 & 20.0 & 20.0 & 10.0 & 50.0 & 70.0 & 40.0 & 1.0 & $10 ; .11$ \\
\hline 93 & 1.0 & 20.0 & 20.0 & 70.0 & 80.0 & 5.0 & 6.0 & 20.0 & 20.0 & 50.0 & 50.0 & 70.0 & 40.0 & 1.0 & 10.11 \\
\hline 94 & 1.0 & 20.0 & 20.0 & 70.0 & 80.0 & 10.0 & 10.0 & 20.0 & 20.0 & 10.0 & 50.0 & 70.0 & 40.0 & 1.0 & 10.11 \\
\hline 95 & 1.0 & 20.0 & 20.0 & 70.0 & 80.0 & 50.0 & 10.0 & 20.0 & 20.0 & 10.0 & 50.0 & 70.0 & 40.0 & 1.0 & 10.0 \\
\hline
\end{tabular}


Table B.4: Exact and approx. results for test suite CS3 (mean error 0.46\%)

\begin{tabular}{|c|c|c|c|c|c|c|c|}
\hline \multirow{3}{*}{ Case } & \multirow{3}{*}{$\begin{array}{c}\text { Type } \\
\text { of } \\
\text { results }\end{array}$} & \multicolumn{6}{|c|}{ Reference task throughputs } \\
\hline & & \multicolumn{2}{|c|}{$T T_{1}$} & \multicolumn{2}{|c|}{$T_{2}$} & \multicolumn{2}{|c|}{$\overline{T_{3}}$} \\
\hline & & thru & err[\%] & thru & err[\%] & thru & err[\%] \\
\hline \multirow[t]{2}{*}{1} & exact & 0.1125 & & 0.1365 & & 0.1247 & \\
\hline & approx & 0.1124 & -0.11 & 0.1369 & 0.31 & 0.1239 & -0.66 \\
\hline \multirow[t]{2}{*}{$\overline{2}$} & exact & 0.1943 & & 0.2123 & & 0.2193 & \\
\hline & approx & 0.1939 & -0.18 & 0.2120 & -0.16 & 0.2195 & 0.09 \\
\hline \multirow[t]{2}{*}{$\overline{3}$} & exact & 0.1383 & & 0.0728 & & 0.0688 & \\
\hline & approx & 0.1360 & -1.66 & 0.0720 & -1.10 & 0.0686 & -0.26 \\
\hline \multirow[t]{2}{*}{4} & exact & 0.1277 & & 0.0533 & & 0.1527 & \\
\hline & approx & 0.1267 & -0.81 & 0.0532 & -0.07 & 0.1533 & 0.38 \\
\hline \multirow[t]{2}{*}{5} & exact & 0.0522 & & 0.0553 & & 0.0560 & \\
\hline & approx & 0.0520 & -0.34 & 0.0552 & -0.02 & 0.0560 & 0.04 \\
\hline \multirow[t]{2}{*}{6} & exact & 0.1087 & & 0.0654 & & 0.1257 & \\
\hline & approx & 0.1078 & -0.82 & 0.0653 & -0.05 & 0.1256 & -0.08 \\
\hline \multirow[t]{2}{*}{7} & exact & 0.3527 & & 0.2473 & & 0.1937 & \\
\hline & approx & 0.3491 & -1.02 & 0.2454 & -0.77 & 0.1925 & -0.59 \\
\hline \multirow[t]{2}{*}{8} & exact & 0.3860 & & 0.2607 & & 0.2012 & \\
\hline & approx & $\mathbf{0 . 3 8 3 4}$ & -0.67 & 0.2590 & -0.63 & 0.2001 & -0.51 \\
\hline \multirow[t]{2}{*}{9} & exact & 0.0669 & & 0.2589 & & 0.2179 & \\
\hline & approx & 0.0669 & -0.06 & 0.2593 & 0.12 & 0.2163 & -0.77 \\
\hline \multirow[t]{2}{*}{10} & exact & 0.0097 & & 0.4127 & & 0.3340 & \\
\hline & approx & 0.0097 & 0.00 & 0.4127 & 0.00 & 0.3337 & -0.08 \\
\hline \multirow[t]{2}{*}{11} & exact & 0.1619 & & 0.1559 & & 0.1426 & \\
\hline & approx & 0.1622 & 0.16 & 0.1553 & -0.40 & 0.1426 & -0.04 \\
\hline \multirow[t]{2}{*}{12} & exact & 0.1779 & & 0.1428 & & 0.2131 & \\
\hline & approx & 0.1772 & -0.43 & 0.1427 & -0.13 & 0.2125 & -0.27 \\
\hline \multirow[t]{2}{*}{13} & exact & 0.1128 & & 0.0857 & & 0.1169 & \\
\hline & approx & 0.1127 & -0.06 & 0.0857 & -0.04 & 0.1110 & 0.09 \\
\hline \multirow[t]{2}{*}{14} & exact & 0.1105 & & 0.1007 & & 0.1130 & \\
\hline & approx & 0.1106 & 0.05 & 0.1004 & -0.35 & 0.1129 & -0.10 \\
\hline \multirow[t]{2}{*}{15} & exact & 0.1993 & & 0.1518 & & 0.1731 & \\
\hline & approx & 0.1994 & 0.07 & 0.1515 & -0.25 & 0.1721 & -0.58 \\
\hline \multirow[t]{2}{*}{16} & exact & 0.0770 & & 0.0940 & & 0.0905 & \\
\hline & approx & 0.0768 & -0.16 & 0.0976 & 3.82 & 0.0881 & -2.64 \\
\hline \multirow[t]{2}{*}{17} & exact & 0.1834 & & 0.2470 & & $0 . \overline{2305}$ & \\
\hline & approx & 0.1825 & -0.48 & 0.2477 & 0.30 & 0.2296 & -0.38 \\
\hline
\end{tabular}

continues on the next page 
Table B.4 continued: CS3 results

\begin{tabular}{|c|c|c|c|c|c|c|c|}
\hline \multirow{3}{*}{ Case } & \multirow{3}{*}{$\begin{array}{c}\text { Type } \\
\text { of } \\
\text { results }\end{array}$} & \multicolumn{6}{|c|}{ Reference task throughputs } \\
\hline & & \multicolumn{2}{|c|}{$\overline{T_{1}}$} & \multicolumn{2}{|c|}{$T_{2}$} & \multicolumn{2}{|c|}{$T$} \\
\hline & & thru & err $[\%]$ & thru & err.\%] & thru & $\operatorname{err}[10]$ \\
\hline 18 & exact & 0.1030 & & 0.1158 & & 0.1160 & \\
\hline 19 & $\begin{array}{l}\text { exact } \\
\text { approx }\end{array}$ & $\begin{array}{l}0.1042 \\
0.1041\end{array}$ & -0.17 & $\begin{array}{l}0.1229 \\
0.1228\end{array}$ & -0.08 & $\begin{array}{l}0.1300 \\
0.1306\end{array}$ & 16 \\
\hline 20 & $\begin{array}{c}\text { exact } \\
\text { approx }\end{array}$ & $\begin{array}{l}0.1080 \\
0.1075\end{array}$ & -0.41 & $\begin{array}{l}0.1272 \\
0.1274\end{array}$ & 0.18 & $\begin{array}{l}0.1265 \\
0.1260\end{array}$ & 0.12 \\
\hline 21 & $\begin{array}{c}\text { exact } \\
\text { approx }\end{array}$ & $\begin{array}{l}0.1925 \\
0.1922\end{array}$ & -0.13 & $\begin{array}{l}0.2882 \\
0.2883 \\
\end{array}$ & 0.0 .1 & $\begin{array}{l}0.2882 \\
0.2883 \\
\end{array}$ & 0.1 \\
\hline 22 & $\begin{array}{c}\text { exact } \\
\text { approx }\end{array}$ & $\begin{array}{l}0.2234 \\
0.2227\end{array}$ & -0.34 & $\begin{array}{l}0.3960 \\
0.3979\end{array}$ & 0.18 & $\begin{array}{l}0.269 .1 \\
0.26 .19\end{array}$ & 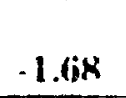 \\
\hline 23 & $\begin{array}{c}\text { exact } \\
\text { approx }\end{array}$ & $\begin{array}{l}0.2027 \\
0.2022\end{array}$ & -0.24 & $\begin{array}{l}0.3192 \\
0.3209 \\
\end{array}$ & 3 & $\begin{array}{l}0.2024 \\
0.2905 \\
\end{array}$ & 0.65 \\
\hline 24 & $\begin{array}{c}\text { exact } \\
\text { approx }\end{array}$ & $\begin{array}{l}0.1965 \\
0.1957\end{array}$ & -0.41 & $\begin{array}{l}0.3274 \\
0.3301\end{array}$ & 33 & $\begin{array}{l}0.2220 \\
0.2201 \\
\end{array}$ & -1.25 \\
\hline$\overline{25}$ & $\begin{array}{c}\text { exact } \\
\text { approx }\end{array}$ & $\begin{array}{l}0.1836 \\
0.1827 \\
\end{array}$ & -0.47 & $\begin{array}{l}0.2459 \\
0.2466 \\
\end{array}$ & 30 & $\begin{array}{l}0.206 .9 \\
0.20 .12 \\
\end{array}$ & 1.30 \\
\hline 26 & $\begin{array}{c}\text { exact } \\
\text { approx }\end{array}$ & $\begin{array}{l}0.1379 \\
0.1374 \\
\end{array}$ & -0.34 & $\begin{array}{l}0.1783 \\
0.1787 \\
\end{array}$ & 22 & $\begin{array}{l}0.1 .187 \\
0.1473 \\
\end{array}$ & 0.93 \\
\hline 27 & $\begin{array}{c}\text { exact } \\
\text { approx }\end{array}$ & $\begin{array}{l}0.1131 \\
0.1130 \\
\end{array}$ & -0.10 & $\begin{array}{l}0.1473 \\
0.1475 \\
\end{array}$ & 10 & $\begin{array}{l}0.1368 \\
0.1205\end{array}$ & -0.95 \\
\hline 28 & $\begin{array}{c}\text { exact } \\
\text { approx }\end{array}$ & $\begin{array}{l}0.1404 \\
0.1402 \\
\end{array}$ & .0 .14 & $\begin{array}{l}0.1524 \\
0.1524 \\
\end{array}$ & 02 & $\begin{array}{l}0.1339 \\
0.1338 \\
\end{array}$ & $-0.0: 3$ \\
\hline 29 & $\begin{array}{c}\text { exact } \\
\text { approx }\end{array}$ & $\begin{array}{l}0.2466 \\
0.2460\end{array}$ & -0.24 & $\begin{array}{l}0.2466 \\
0.2460 \\
\end{array}$ & 4 & $\begin{array}{l}0.20355 \\
0.2035\end{array}$ & 0.010 \\
\hline 30 & $\begin{array}{c}\text { exact } \\
\text { approx }\end{array}$ & $\begin{array}{l}0.2286 \\
0.2269 \\
\end{array}$ & -0.75 & $\begin{array}{l}0.2198 \\
0.2200 \\
\end{array}$ & 10 & $\begin{array}{l}0.1098 \\
0.1087 \\
\end{array}$ & -0.52 \\
\hline$\overline{31}$ & $\begin{array}{c}\text { exact } \\
\text { approx }\end{array}$ & $\begin{array}{l}0.3131 \\
0.3139 \\
\end{array}$ & 0.20 & $\begin{array}{l}0.2800 \\
0.2786 \\
\end{array}$ & -0.50 & $\begin{array}{r}.2391 \\
0.2383 \\
\end{array}$ & $-(1.35$ \\
\hline 32 & $\begin{array}{c}\text { exact } \\
\text { approx }\end{array}$ & $\begin{array}{l}0.2070 \\
0.2058\end{array}$ & -0.56 & $\begin{array}{l}0.3356 \\
0.3336 \\
\end{array}$ & -0.59 & $\begin{array}{l}0.2746 \\
0.2724 \\
\end{array}$ & -0.81 \\
\hline$\overline{33}$ & $\begin{array}{c}\text { exact } \\
\text { approx }\end{array}$ & $\begin{array}{l}0.2194 \\
0.2182 \\
\end{array}$ & -0.57 & $\begin{array}{l}0.2630 \\
0.2637 \\
\end{array}$ & 0.28 & $\begin{array}{l}0.2386 \\
0.2364 \\
\end{array}$ & -0.94 \\
\hline 34 & $\begin{array}{c}\text { exact } \\
\text { approx }\end{array}$ & $\begin{array}{l}0.0863 \\
0.0862\end{array}$ & -0.18 & $\begin{array}{l}0.1218 \\
0.1224 \\
\end{array}$ & 0.44 & $\begin{array}{l}0.1152 \\
0.1133 \\
\end{array}$ & -1.655 \\
\hline 35 & $\begin{array}{c}\text { exact } \\
\text { approx }\end{array}$ & $\begin{array}{l}0.1124 \\
0.1121 \\
\end{array}$ & -0.26 & $\begin{array}{l}0.1964 \\
0.1968 \\
\end{array}$ & 0.21 & $\begin{array}{l}0.1828 \\
0.1803 \\
\end{array}$ & -1.35 \\
\hline
\end{tabular}


Table B.5: Exact and approx. results for test suite CS4(mean error 0.53\%)

\begin{tabular}{|c|c|c|c|c|c|c|c|c|c|}
\hline \multirow{3}{*}{ Case } & \multirow{3}{*}{$\begin{array}{c}\text { Type } \\
\text { of } \\
\text { results }\end{array}$} & \multicolumn{8}{|c|}{ Reference task throughputs } \\
\hline & & \multicolumn{2}{|c|}{$\bar{T}$} & \multicolumn{2}{|c|}{$T_{2}$} & \multicolumn{2}{|c|}{$T_{3}$} & \multicolumn{2}{|c|}{$T_{4}$} \\
\hline & & thru & $\operatorname{err}[\%]$ & thru & $\operatorname{err}[\%]$ & thru & err[\%] & thru & $\operatorname{err}[\%$ \\
\hline 1 & exact & $\begin{array}{l}0.0924 \\
0.0923\end{array}$ & -0.07 & $\begin{array}{l}0.1042 \\
0.1043\end{array}$ & 008 & 0.0984 & & 0.0968 & \\
\hline \multirow[t]{2}{*}{2} & exact & 0.1002 & & 0.1153 & & \begin{tabular}{|c|}
0.0902 \\
\end{tabular} & -0.36 & \begin{tabular}{|l|l|}
0.0569 \\
0.1103 \\
\end{tabular} & 0.09 \\
\hline & approx & 0.1002 & -0.08 & 0.1151 & -0.19 & 0.1074 & -0.49 & 0.1108 & 0.49 \\
\hline \multirow[t]{2}{*}{3} & exact & 0.0677 & & 0.1059 & & 0.1073 & & 0.1031 & \\
\hline & approx & 0.0673 & -0.61 & 0.1039 & -1.89 & 0.1055 & -1.66 & 0.1018 & -1.31 \\
\hline 4 & $\begin{array}{l}\text { exact } \\
\text { approx }\end{array}$ & $\begin{array}{l}0.2037 \\
0.2013\end{array}$ & -1.17 & $\begin{array}{l}0.2037 \\
0.2013\end{array}$ & -1.17 & $\begin{array}{l}0.2037 \\
0.2013\end{array}$ & -1.17 & $\begin{array}{l}0.2037 \\
0.2013\end{array}$ & -1.17 \\
\hline \multirow[t]{2}{*}{5} & exact & 0.2295 & & 0.2295 & & 0.2295 & & 0.3805 & \\
\hline & approx & 0.2269 & -1.15 & 0.2269 & -1.15 & 0.2269 & -1.15 & 0.3790 & -0.39 \\
\hline \multirow[t]{2}{*}{6} & exact & 0.0611 & & 0.0576 & & 0.0547 & & 0.0520 & \\
\hline & approx & 0.0613 & 0.32 & 0.0575 & -0.24 & 0.0544 & -0.42 & 0.0518 & -0.41 \\
\hline 7 & \begin{tabular}{|c|} 
exact \\
approx
\end{tabular} & 0.1351 & ก?90 & 0.1420 & 009 & 0.1694 & & 0.1645 & 0.5 \\
\hline \multirow[t]{2}{*}{8} & exact & 0.1264 & -0.65 & \begin{tabular}{|l|}
0.1411 \\
0.1338 \\
\end{tabular} & -0.03 & \begin{tabular}{|l|}
0.1684 \\
0.1338 \\
\end{tabular} & 0.61 & \begin{tabular}{|l}
0.1647 \\
0.1281 \\
\end{tabular} & \\
\hline & approx & 0.1252 & -0.95 & 0.1323 & -1.14 & 0.1323 & -1.14 & 0.1270 & -0.87 \\
\hline \multirow[t]{2}{*}{9} & exact & 0.1446 & & 0.1925 & & 0.1776 & & 0.2291 & \\
\hline & approx & 0.1444 & -0.14 & 0.1920 & -0.25 & 0.1769 & -0.37 & 0.2306 & 0.66 \\
\hline \multirow[t]{2}{*}{10} & exact & 0.1113 & & 0.1103 & & 0.1031 & & 0.0930 & \\
\hline & approx & 0.1125 & 1.10 & 0.1088 & -1.32 & 0.1031 & 0.01 & 0.0925 & -0.57 \\
\hline \multirow[t]{2}{*}{11} & exact & 0.1130 & & 0.1103 & & 0.1042 & & \begin{tabular}{|c|c|}
0.0938 \\
\end{tabular} & \\
\hline & approx & 0.1131 & 0.09 & 0.1103 & -0.05 & 0.1041 & -0.05 & 0.0938 & -0.09 \\
\hline \multirow[t]{2}{*}{12} & exact & 0.1149 & & 0.1001 & & 0.1276 & & 0.1188 & \\
\hline & approx & 0.1146 & -0.25 & 0.1000 & -0.05 & 0.1280 & 0.25 & 0.1185 & -0.26 \\
\hline \multirow[t]{2}{*}{13} & exact & 0.0843 & & 0.0695 & & 0.0834 & & $\overline{0.0793}$ & \\
\hline & approx & 0.0842 & -0.15 & 0.0695 & 0.03 & 0.0838 & 0.49 & 0.0794 & 0.05 \\
\hline \multirow[t]{2}{*}{14} & exact & 0.0836 & & 0.0783 & & 0.0848 & & \begin{tabular}{|l|}
0.0809 \\
\end{tabular} & \\
\hline & approx & 0.0837 & 0.11 & 0.0779 & -0.45 & 0.0851 & 0.25 & 0.0807 & -0.21 \\
\hline \multirow[t]{2}{*}{15} & exact & 0.1558 & & \begin{tabular}{|l}
0.1273 \\
\end{tabular} & & 0.1411 & & \begin{tabular}{|l|l|}
0.1336 \\
\end{tabular} & \\
\hline & approx & 0.1562 & 0.27 & 0.1271 & -0.22 & 0.1399 & -0.80 & 0.1333 & -0.21 \\
\hline \multirow[t]{2}{*}{16} & exact & 0.0981 & & 0.1182 & & 0.1147 & & 0.1095 & \\
\hline & approx & 0.0982 & 0.04 & $0.1 \div 78$ & -0.34 & 0.1141 & -0.55 & 0.1096 & 0.12 \\
\hline 17 & $\begin{array}{l}\text { exact } \\
\text { approx }\end{array}$ & $\begin{array}{l}0.1365 \\
0.1358\end{array}$ & -0.54 & $\begin{array}{l}0.16 \overline{41} \\
0.1646\end{array}$ & 0.33 & $\begin{array}{l}0.1579 \\
0.1577\end{array}$ & -0.12 & $\begin{array}{l}0.1492 \\
0.1494\end{array}$ & 0.10 \\
\hline & & & & & & & 0.12 & & \\
\hline
\end{tabular}

continues on the next page 
Table B.5 continued: CS4 res:alts

\begin{tabular}{|c|c|c|c|c|c|c|c|c|c|}
\hline \multirow{3}{*}{ Case } & \multirow{3}{*}{$\begin{array}{c}\text { Type } \\
\text { of } \\
\text { results }\end{array}$} & \multicolumn{8}{|c|}{ Reference task throughputs } \\
\hline & & \multicolumn{2}{|c|}{$\overline{T_{1}}$} & \multicolumn{2}{|c|}{$T_{2}$} & \multicolumn{2}{|c|}{$\overline{T_{3}}$} & \multicolumn{2}{|c|}{$T_{4}$} \\
\hline & & thru & err[\%] & thru & $\operatorname{err}[\%]$ & thru & err[\%] & thru & $\operatorname{crr}[\%]$ \\
\hline \multirow[t]{2}{*}{18} & exact & $0.086 \bar{\delta}$ & & 0.0967 & & 0.0948 & & 0.0918 & \\
\hline & approx & 0.0860 & -0.68 & 0.0973 & 0.60 & 0.0942 & -0.71 & 0.0915 & -0.37 \\
\hline \multirow[t]{2}{*}{19} & exact & 0.0920 & & 0.1045 & & 0.1092 & & 0.0962 & \\
\hline & approx & 0.0918 & -0.16 & 0.1053 & 0.79 & 0.1085 & .0 .64 & 0.0962 & -0.01 \\
\hline \multirow[t]{2}{*}{20} & exact & 0.0946 & & 0.1072 & & 0.1072 & & 0.0992 & \\
\hline & approx & 0.0940 & -0.65 & 0.1077 & 0.48 & 0.1065 & -0.67 & 0.0987 & .0 .45 \\
\hline \multirow[t]{2}{*}{$\overline{21}$} & exact & 0.1547 & & 0.2045 & & 0.2045 & & 0.1671 & \\
\hline & approx & 0.1543 & -0.31 & 0.2052 & 0.36 & 0.2052 & 0.36 & 0.1666 & -0.27 \\
\hline \multirow[t]{2}{*}{22} & exact & 0.1746 & & 0.2499 & & 0.1967 & & 0.1912 & \\
\hline & approx & 0.1735 & -0.62 & 0.2535 & 1.41 & 0.1936 & -1.61 & 0.1904 & -0.43 \\
\hline \multirow[t]{2}{*}{$\overline{23}$} & exact & 0.1611 & & 0.2180 & & 0.2066 & & 0.1748 & \\
\hline & approx & 0.1604 & -0.41 & 0.2201 & 0.94 & 0.2060 & -0.30 & 0.1743 & -0.26 \\
\hline \multirow[t]{2}{*}{24} & exact & 0.1559 & & 0.2162 & & 0.1694 & & 0.1721 & \\
\hline & appro\% & 0.1552 & -0.46 & 0.2191 & 1.33 & 0.1677 & -1.02 & 0.1716 & .0 .30 \\
\hline \multirow[t]{2}{*}{25} & exact & 0.1463 & & 0.1778 & & 0.1583 & & 0.160 .4 & \\
\hline & approx & 0.1455 & -0.53 & 0.1791 & 0.76 & 0.1564 & .1 .14 & 0.1600 & -0.22 \\
\hline \multirow[t]{2}{*}{$\overline{26}$} & exact & 0.1166 & & 0.1 .405 & & 0.1234 & & 0.1253 & \\
\hline & арprox & 0.1162 & -0.42 & 0.1412 & 0.48 & 0.1223 & -0.94 & 0.1250 & -0.26 \\
\hline \multirow[t]{2}{*}{27} & exact & 0.0981 & & $0.119 \overline{3}$ & & 0.1097 & & 0.1079 & \\
\hline & approx & 0.0981 & -0.04 & 0.1196 & 0.23 & 0.1085 & -1.10 & 0.1077 & .0 .16 \\
\hline \multirow[t]{2}{*}{28} & exact & 0.1177 & & $0.125 n$ & & 0.1133 & & 0.1110 & \\
\hline & approx & 0.1175 & -0.19 & 0.1248 & -0.16 & 0.1136 & 0.22 & 0.1113 & 0.20 \\
\hline \multirow[t]{2}{*}{$\overline{29}$} & exact & 0.1846 & & 0.1846 & & 0.1608 & & 0.1567 & \\
\hline & approx & 0.1834 & -0.65 & 0.18344 & -0.65 & 0.1614 & 0.36 & 0.1573 & $0.3 x$ \\
\hline \multirow[t]{2}{*}{30} & exact & 0.1718 & & 0.1671 & & 0.1562 & & 0.1590 & \\
\hline & approx & 0.1709 & -0.55 & 0.1680 & 0.54 & 0.1553 & -0.58 & 0.1583 & .0 .16 \\
\hline \multirow[t]{2}{*}{31} & exact & 0.2175 & & 0.2026 & & 0.1814 & & 0.1854 & \\
\hline & approx & 0.2189 & 0.61 & 0.2020 & -0.31 & 0.1807 & -0.38 & 0.1848 & -0.32 \\
\hline \multirow[t]{2}{*}{32} & exart & 0.1602 & & 0.2235 & & 0.1967 & & 0.2011 & \\
\hline & approx & 0.1594 & -0.55 & 0.2234 & -0.04 & O 1955 & -0.64 & 0.2001 & -0.53 \\
\hline \multirow[t]{2}{*}{33} & exact & 0.1682 & & 0.1889 & & 0.1774 & & 0.1727 & \\
\hline & approx & 0.1672 & -0.58 & 0.1901 & 0.64 & 0.1760 & -0.78 & 0.1725 & $-(0.09)$ \\
\hline \multirow[t]{2}{*}{34} & exact & 0.0769 & & 0.1001 & & 0.0963 & & 0.0946 & \\
\hline & approx & 0.0767 & -0.23 & 0.1011 & 0.96 & 0.0949 & -1.44 & 0.09399 & -0.77 \\
\hline \multirow[t]{2}{*}{35} & cet & $0.0 ; 72$ & & 0.1504 & & 0.1433 & & 0.1405 & \\
\hline & 理 & 0.0969 & -0.31 & 0.1516 & 0.75 & 0.1412 & -1.52 & 0.1394 & -(1).78 \\
\hline
\end{tabular}



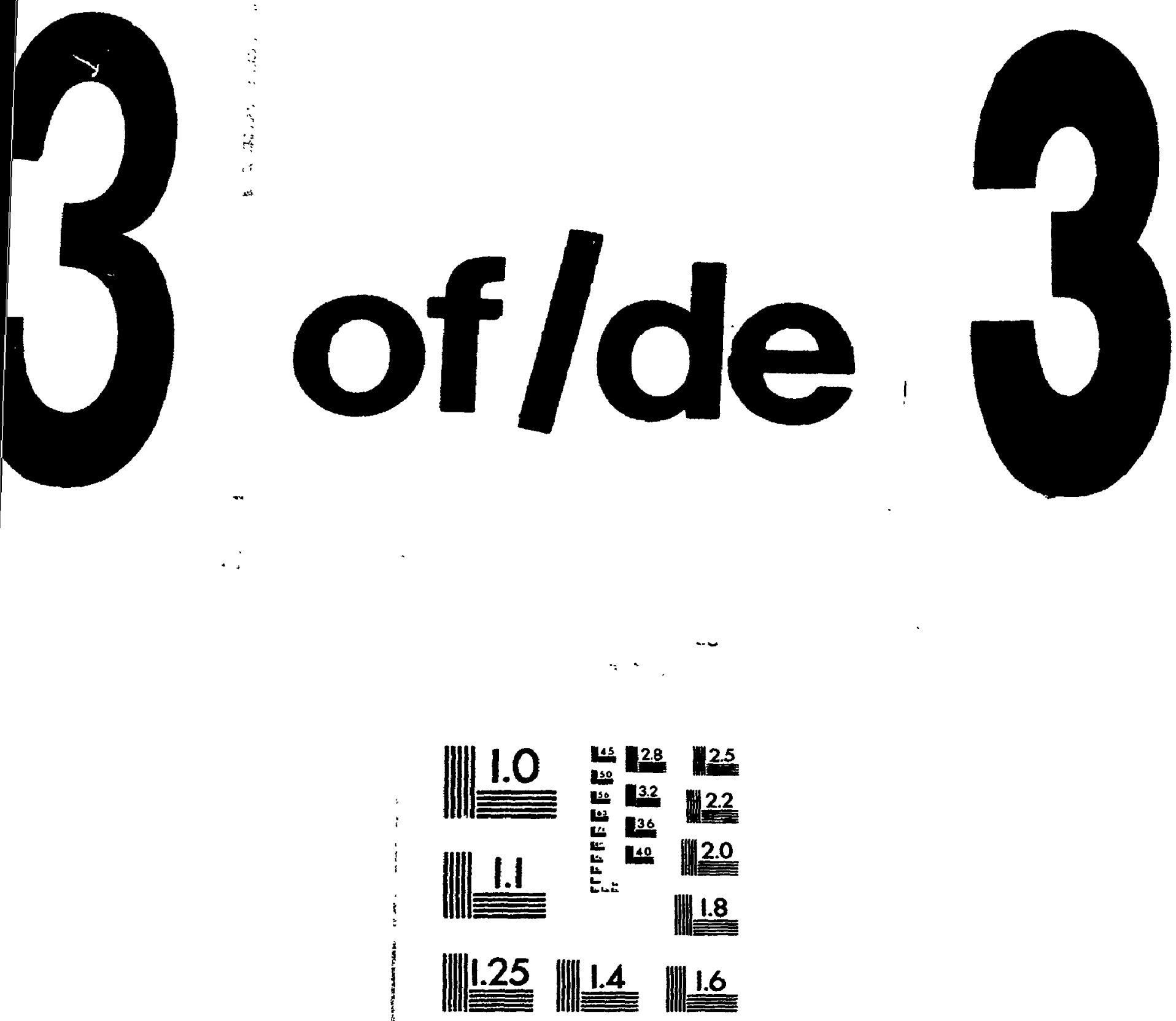

MICROCOPY RESOLUTION TEST CHART

NATIOHAL BUREAU OF STANDARDS

STANDARD REFERENCE MATEPIAL YO10a

(ANSI and ISO TEST CHART No.2) 
Table B.6: Exact and approximate results for test suite CS5 (mean error 0.65\%)

\begin{tabular}{|c|c|c|c|c|c|c|c|c|c|c|c|}
\hline \multirow{3}{*}{ Case } & \multirow{3}{*}{$\begin{array}{l}\text { Type } \\
\text { of } \\
\text { results }\end{array}$} & \multicolumn{10}{|c|}{ Reference task throughputs } \\
\hline & & \multicolumn{2}{|c|}{$T_{1}$} & \multicolumn{2}{|c|}{$T_{2}$} & \multicolumn{2}{|c|}{ T. } & \multicolumn{2}{|c|}{$\overline{T_{4}}$} & \multicolumn{2}{|c|}{$T_{5}$} \\
\hline & & thru & $\mathrm{r} \%$ & thru & $\mathbf{x} \%$ & thru & $\mathrm{rr} \%$ & thru & err & thru & err \\
\hline \multirow[t]{2}{*}{ I } & exact & 0.0781 & & 0.0853 & & 0.0818 & & 0.0808 & & 808 & \\
\hline & rox & 82 & 0.00 & .0850 & -0.37 & & -0.34 & 0809 & 0.18 & 309 & 0.18 \\
\hline \multirow[t]{2}{*}{$\overline{2}$} & exact & 0.0862 & & 0.0950 & & 0.0 & & 0.0927 & & .0937 & \\
\hline & prox & 0.0862 & -0.07 & 0.0951 & 0.07 & 04 & -0.28 & .0929 & 22 & .0937 & .07 \\
\hline \multirow[t]{2}{*}{$\overline{3}$} & exact & 0.0522 & & 0.0692 & & 0.0702 & & 0.0688 & & & \\
\hline & approx & 19 & -0.69 & 0.0678 & -2.13 & & -1.90 & 0.0678 & -1.48 & 0.0917 & -0.1 .1 \\
\hline \multirow[t]{2}{*}{$\overline{4}$} & exact & 0.0820 & & 0.0698 & & & & 0.0592 & & & \\
\hline & approx & 0.0822 & 0.22 & 0.0691 & -1.04 & 0.0 & -0.83 & 0.0590 & -0.41 & & $-(0.15$ \\
\hline \multirow[t]{2}{*}{5} & exact & 0.0561 & & 0.0533 & & & & 0.0487 & & 0.0468 & \\
\hline & approx & 0.0567 & 1.01 & 0.0533 & 0.11 & & -0.21 & 0.0486 & -0.31 & 166 & -0.30 \\
\hline \multirow[t]{2}{*}{$\overline{6}$} & exact & 0.0549 & & 0.0518 & & & & 0.0450 & & & \\
\hline & approx & 0.0551 & 0.51 & 0.0516 & -0.26 & 0.0 & -0.28 & 0.0448 & -0.31 & 16 & -0.37 \\
\hline \multirow[t]{2}{*}{7} & exact & 0.0370 & & 0.0 & & & & 0.0 & & & \\
\hline & prox & 0.0365 & 1.51 & 0.0 & -0.32 & & 0.10 & & 20 & & .17 \\
\hline \multirow[t]{2}{*}{8} & exact & 0.0611 & & 0.0553 & & & & 177 & & & \\
\hline & prox & 0. & .35 & & .62 & & 01 & & 22 & & , \\
\hline \multirow[t]{2}{*}{$\overline{9}$} & exact & 0.1 & & & & & & & & & \\
\hline & approx & & .12 & & .34 & & 0.35 & & & & . \\
\hline \multirow[t]{2}{*}{10} & exact & 0.1 & & & & & & $\overline{32}$ & & & \\
\hline & prox & 0.1114 & -1.63 & 39 & -1.93 & & -1.93 & 14 & -1.63 & & .5 \\
\hline \multirow[t]{2}{*}{11} & exact & 0.0904 & & & & & & & & & \\
\hline & prox & 0.0907 & 0.34 & 92 & -0.65 & & 0.15 & 83 & -0.48 & & 0.07 \\
\hline \multirow[t]{2}{*}{12} & exact & 0.0838 & & 0.0822 & & & & 0.0735 & & & \\
\hline & prox & & -0.62 & & -0.02 & & 0.09 & & -0.02 & & 16 \\
\hline \multirow[t]{2}{*}{13} & exact & 0.1235 & & 0.1071 & & & & & & & \\
\hline & approx & 0.1233 & 0.14 & & 0 & & $\mathbf{U}$ & & & & -10.72 \\
\hline \multirow[t]{2}{*}{14} & exact & 0.0 & & 26 & & & & $\overline{23}$ & & & \\
\hline & approx & & .06 & & 0.07 & & 58 & & .34 & & 0.24 \\
\hline 15 & exact & 0.0860 & & 0.0817 & & & & 327 & & 90 & \\
\hline & approx & & 0.29 & & -0.38 & & 0.25 & 22 & -0.62 & & $-0.6 \%$ \\
\hline 16 & exact & 0.1240 & & 0.1111 & & 0. & & 0.1172 & & 111 & \\
\hline & prox & 254 & 1.05 & & -0.52 & & -0.56 & & .27 & & $0.5^{4}$ \\
\hline 17 & exact & 0.1255 & & 0.1110 & & 0.1184 & & 0.1130 & & 110 & \\
\hline & approx & 0.1263 & 0.69 & & 2 & & -0.47 & & .031 & & $0.4^{*}$ \\
\hline 18 & exact & 0.2073 & & 0.2073 & & 0.2 & & 0.3119 & & & \\
\hline & rox & & .07 & & -1.07 & & -1.07 & & -0.07 & & 0.07 \\
\hline$\overline{19}$ & exact & 0.1658 & & 0.1658 & & & & & & & \\
\hline & appion & & -0.96 & & -0.96 & & -0.96 & 43 & -0.96 & & $\cdots$ \\
\hline 20 & exact & 0.1000 & & 0.1000 & & 0.1000 & & 0.1000 & & 0.1000 & \\
\hline & rox & 0.1000 & 0.00 & 0.1000 & 0.0 & 0.1000 & 0.00 & 0.1000 & 0.00 & 0.1000 & 0.0 \\
\hline
\end{tabular}

continues on the next page 
CS5 results continued

\begin{tabular}{|c|c|c|c|c|c|c|c|c|c|c|c|}
\hline \multirow{3}{*}{ Case } & \multirow{3}{*}{$\begin{array}{l}\text { Type } \\
\text { of } \\
\text { results }\end{array}$} & \multicolumn{10}{|c|}{ Reference task throughputs } \\
\hline & & \multicolumn{2}{|c|}{$\overline{T_{1}}$} & \multicolumn{2}{|c|}{$\overline{T_{2}}$} & \multicolumn{2}{|c|}{$T_{3}$} & \multicolumn{2}{|c|}{$\overline{T_{1}}$} & \multicolumn{2}{|c|}{$T_{5}$} \\
\hline & & thru & $r \%$ & thru & err\% & thru & $\mathrm{r} \%$ & thru & $\mathrm{rr} \%$ & thru & $2 \pi \%$ \\
\hline \multirow[t]{2}{*}{$\overline{21}$} & exact & 0.0991 & & 0.1264 & & 0.1114 & & 0.1057 & & 0.1188 & \\
\hline & approx & 0.0992 & 0.13 & 0.1266 & 0.17 & 0.1110 & -0.40 & 0.1059 & 0.17 & 0.1183 & -0.40 \\
\hline \multirow[t]{2}{*}{22} & exact & 0.1225 & & 0.1608 & & 0.1388 & & 0.1301 & & 0.1500 & \\
\hline & approx & 0.1219 & -0.44 & 0.1607 & -0.03 & 0.1385 & -0.19 & 0.1301 & 0.02 & 0.1503 & 0.23 \\
\hline \multirow[t]{2}{*}{23} & exact & 0.1338 & & 0.1858 & & 0.1537 & & 0.1424 & & 1676 & \\
\hline & prox & 0.1330 & -0.59 & 0.1839 & -0.99 & 0.1532 & -0.29 & 0.1424 & -0.03 & 1684 & .47 \\
\hline \multirow[t]{2}{*}{24} & $\overline{\text { exact }}$ & 0.1051 & & 0.1397 & & 0.1224 & & 0.1153 & & $\longdiv { 1 3 1 4 }$ & \\
\hline & approx & 0.1053 & 0.10 & 0.1392 & -0.40 & 0.1219 & -0.43 & 0.1155 & 0.15 & 1313 & -0.06 \\
\hline \multirow[t]{2}{*}{25} & exact & 0.1094 & & 0.1406 & & 0.1262 & & 0.1192 & & 353 & \\
\hline & approx & 0.1091 & -0.24 & .1415 & 0.60 & 0.1257 & -0.42 & 0.1191 & -0.05 & 351 & -0.17 \\
\hline \multirow[t]{2}{*}{26} & exact & 0.1007 & & 0.1180 & & 0.1165 & & 0.1098 & & 0.1243 & \\
\hline & approx & 0.1007 & $0 . \overline{0} \bar{T}$ & 0.1176 & -0.34 & 0.1158 & -0.61 & 0.1099 & 0.12 & 0.1243 & 0.03 \\
\hline \multirow[t]{2}{*}{27} & exact & 0.1046 & & 0.1189 & & 0.1142 & & 0.1077 & & 0.1224 & \\
\hline & approx & 1044 & -0.17 & 0.1184 & -0.45 & 0.1140 & -0.19 & 0.1078 & 0.07 & 0.1229 & 0.42 \\
\hline \multirow[t]{2}{*}{28} & exact & 0.1006 & & 0.1128 & & 0.1200 & & 0.1158 & & 0.1160 & \\
\hline & approx & 0.1005 & -0.11 & 0.1124 & -0.33 & 0.1199 & -0.04 & 0.1161 & .27 & 0.1160 & -0.01 \\
\hline \multirow[t]{2}{*}{29} & exact & 0.1489 & & 0.1844 & & 0.2094 & & 0.1972 & & 0.1969 & \\
\hline & prox & 1486 & -0.21 & 0.1830 & -0.75 & 0.2095 & .06 & 0.1977 & .23 & 966 & .13 \\
\hline \multirow[t]{2}{*}{30} & exact & 0.1075 & & $\overline{0.1}$ & & $\overline{0.1}$ & & 0.1 & & 7 & \\
\hline & trox & 1073 & .16 & & -0.74 & 0. & .05 & & 69 & & .06 \\
\hline \multirow[t]{2}{*}{31} & act & 0.0926 & & 61 & & 0.1148 & & 06 & & & \\
\hline & rox & 26 & .04 & & 0.25 & 0. & .13 & & .27 & & 0.25 \\
\hline \multirow[t]{2}{*}{32} & exact & 0.0771 & & 0.0800 & & 0.0834 & & & & 20 & \\
\hline & rox & 72 & 0.16 & & 20 & 0.0 & -0.10 & & -1.35 & 320 & .08 \\
\hline \multirow[t]{2}{*}{33} & exact & 0.0829 & & 0.0870 & & 0.0916 & & 0.0953 & & 0.0903 & \\
\hline & approx & 0.0824 & .68 & & -0.80 & & -0.26 & 0.0957 & .48 & 03 & .07 \\
\hline \multirow[t]{2}{*}{34} & exact & 0.1058 & & 0.1127 & & 0.1220 & & 0.1303 & & 0.1200 & \\
\hline & approx & & 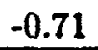 & & 80 & & -0.00 & & ter & & .05 \\
\hline$\overline{35}$ & exact & 0.1084 & & 0.1153 & & 0.1224 & & 0.1298 & & 0.1188 & \\
\hline & rox & & 02 & & -1.24 & & 46 & & $? .69$ & 89 & .03 \\
\hline 36 & act & 0.0438 & & 0.0532 & & 0.0540 & & 0.0530 & & 884 & \\
\hline & approx & & .61 & & 25 & & -1.99 & & $-1.5 \downarrow$ & 86 & .38 \\
\hline 37 & exact & 0.0318 & & 0.0349 & & 0.0354 & & 0.0349 & & 0.0433 & \\
\hline & apprnx & & .0 .42 & & 13 & & 1.89 & & 52 & & .97 \\
\hline 38 & exact & 0.0585 & & 0.0864 & & 0.0877 & & 0.0855 & & 0.1202 & \\
\hline & appivs & & -0.74 & & -1.85 & 0.0862 & -1.63 & 0.0845 & -1.24 & 0.1195 & -0.63 \\
\hline 39 & exact & 0.0426 & & 0.0589 & & 0.0598 & & $0.05 \overline{87}$ & & 0.0758 & \\
\hline & rox & 0.0425 & -0.35 & 0.0574 & -2.66 & & -2.37 & 0.0576 & -1.84 & 0.0756 & .28 \\
\hline 40 & exact & 0.0349 & & 0.0494 & & 0.0500 & & 0.0491 & & 324 & \\
\hline & rox & 48 & -0.25 & & -3.04 & & -2.70 & 81 & -2.09 & 0621 & 39 \\
\hline
\end{tabular}
continues on the next page 
CS5 results continued

\begin{tabular}{|c|c|c|c|c|c|c|c|c|c|c|c|}
\hline \multirow{3}{*}{ Case } & \multirow{3}{*}{$\begin{array}{c}\text { Type } \\
\text { of } \\
\text { results }\end{array}$} & \multicolumn{10}{|c|}{ Reference task throughputs } \\
\hline & & \multicolumn{2}{|c|}{$T T_{1}$} & \multicolumn{2}{|c|}{$\overline{T_{2}}$} & \multicolumn{2}{|c|}{$T_{3}$} & \multicolumn{2}{|c|}{$T_{4}$} & \multicolumn{2}{|c|}{$T T_{5}$} \\
\hline & & thru & err\% & thru & err\% & thru & err\% & thru & err\% & thru & err\% \\
\hline \multirow[t]{2}{*}{$\overline{41}$} & exact & 0.0258 & & 0.0371 & & 0.0376 & & 0.0369 & & 0.0462 & \\
\hline & approx & 0.0258 & -0.18 & 0.0358 & -3.38 & 0.0364 & -2.99 & 0.0361 & -2.28 & 0.0460 & -0.46 \\
\hline \multirow[t]{2}{*}{42} & exact & 0.0500 & & 0.0500 & & 0.0500 & & 0.0500 & & 0.0500 & \\
\hline & approx & 0.0502 & 0.42 & 0.0502 & 0.42 & 0.0502 & 0.42 & 0.0502 & 0.42 & 0.0502 & 0.42 \\
\hline \multirow[t]{2}{*}{43} & exact & 0.0500 & & 0.0500 & & 0.0500 & & 0.0500 & & 0.0500 & \\
\hline & approx & 0.0501 & 0.16 & 0.0501 & 0.16 & 0.0501 & 0.16 & 0.0501 & 0.16 & 0.0501 & 0.16 \\
\hline \multirow[t]{2}{*}{44} & exact & 0.0333 & & 0.0333 & & $\overline{0.0333}$ & & $0 . \overline{0333}$ & & 0.0333 & \\
\hline & approx & 0.0334 & 0.15 & 0.0334 & 0.15 & 0.0334 & 0.15 & 0.0334 & 0.15 & 0.0334 & 015 \\
\hline \multirow[t]{2}{*}{45} & exact & 0.0997 & & 0.0997 & & 0.0997 & & 0.0997 & & 0.0997 & \\
\hline & approx & 0.0990 & -0.72 & 0.0990 & -0.72 & 0.0990 & -0.72 & 0.0990 & -0.72 & 0.0990 & -0.72 \\
\hline \multirow[t]{2}{*}{46} & exact & 0.0500 & & 0.0500 & & 0.0500 & & 0.0500 & & 0.0500 & \\
\hline & approx & 0.0500 & -0.08 & 0.0500 & -0.08 & 0.0500 & -0.08 & 0.0500 & -0.08 & 0.0500 & -0.08 \\
\hline \multirow[t]{2}{*}{47} & exact & 0.0500 & & 0.0500 & & 0.0500 & & 0.0500 & & 0.0500 & \\
\hline & approx & 0.0499 & -0.14 & 0.0499 & -0.14 & 0.0499 & -0.14 & 0.0499 & -0.14 & 0.0499 & -0.14 \\
\hline \multirow[t]{2}{*}{48} & exact & 0.0333 & & 0.0333 & & 0.0333 & & 0.0333 & & 0.0333 & \\
\hline & approx & 0.0333 & 0.00 & 0.0333 & 0.00 & 0.0333 & 0.00 & 0.0333 & 0.00 & 0.0333 & 0.00 \\
\hline \multirow[t]{2}{*}{49} & exact & 0.0766 & & 0.0766 & & 0.0766 & & 0.0766 & & 0.0766 & \\
\hline & approx & 0.0760 & -0.79 & 0.0760 & -0.79 & 0.0760 & -0.79 & 0.0760 & -0.79 & 0.0760 & -0.79 \\
\hline \multirow[t]{2}{*}{$\overline{50}$} & exact & 0.0479 & & 0.0479 & & 0.0479 & & 0.0479 & & 0.0479 & \\
\hline & approx & 0.0470 & -1.94 & 0.0470 & -1.94 & 0.0470 & -1.94 & 0.0470 & -1.94 & 0.0470 & -1.94 \\
\hline \multirow[t]{2}{*}{51} & exact & 0.0485 & & 0.0485 & & 0.0485 & & 0.0485 & & 0.0485 & \\
\hline & approx & 0.0477 & -1.65 & 0.0477 & -1.65 & 0.0477 & -1.65 & 0.0477 & -1.65 & 0.0477 & -1.65 \\
\hline \multirow[t]{2}{*}{52} & exact & 0.0099 & & 0.0099 & & 0.0099 & & 0.0099 & & 0.0099 & \\
\hline & approx & 0.0099 & 0.00 & 0.0099 & 0.00 & 0.0099 & 0.00 & 0.0099 & 0.00 & 0.0099 & 0.00 \\
\hline \multirow[t]{2}{*}{$\overline{53}$} & exact & 0.0087 & & 0.0087 & & 0.0087 & & 0.0087 & & 0.0087 & \\
\hline & approx & 0.0087 & -0.14 & 0.0087 & -0.14 & 0.0087 & -0.14 & 0.0087 & -0.14 & 0.0087 & -0.14 \\
\hline \multirow[t]{2}{*}{54} & exact & 0.0092 & & 0.0092 & & 0.0092 & & 0.0092 & & 0.0092 & \\
\hline & approx & 0.0092 & -0.11 & 0.0092 & -0.11 & 0.0092 & -0.11 & 0.0092 & -0.11 & 0.0092 & -0.11 \\
\hline 55 & exact & 0.0698 & & 0.0680 & & 0.0664 & & 0.0650 & & 0.0626 & \\
\hline & approx & 0.0685 & -1.93 & 0.0669 & -1.68 & 0.0654 & -1.47 & 0.0642 & -1.29 & 0.0619 & -1.03 \\
\hline 56 & exact & 0.0593 & & 0.0585 & & 0.0577 & & 0.0570 & & 0.0559 & \\
\hline & approx & 0.0578 & -2.58 & 0.0573 & -2.07 & 0.0567 & -1.73 & 0.0562 & -1.48 & 0.0552 & -1.17 \\
\hline$\overline{57}$ & exact & 0.0750 & & 0.0681 & & 0.0624 & & 0.0578 & & 0.0533 & \\
\hline & approx & 0.0723 & -3.64 & 0.0663 & -2.57 & 0.0612 & -1.93 & 0.0569 & -1.53 & 0.0527 & -1.12 \\
\hline 58 & exact & 0.0521 & & 0.0547 & & 0.0577 & & 0.0613 & & 0.0650 & \\
\hline & approx & 0.0510 & -2.22 & 0.0537 & -1.87 & 0.0568 & -1.61 & 0.0604 & -1.42 & 0.0643 & -1.11 \\
\hline$\overline{59}$ & exact & 0.0630 & & 0.0627 & & 0.0624 & & 0.0821 & & 0.0617 & \\
\hline & approx & 0.0615 & -2.47 & 0.0617 & -1.65 & 0.0617 & -1.14 & 0.0616 & -0.81 & 0.0614 & -0.14 \\
\hline 60 & exact & 0.0700 & & 0.0668 & & 0.0641 & & 0.0616 & & 0.0692 & \\
\hline & approx & 0.0683 & -2.37 & 0.0659 & -1.34 & 0.0636 & .0 .79 & 0.0613 & -0.49 & 0.0591 & -0.21 \\
\hline
\end{tabular}

continues on the next page 
CS5 results continued

\begin{tabular}{|c|c|c|c|c|c|c|c|c|c|c|c|}
\hline \multirow{3}{*}{ Case } & \multirow{3}{*}{$\begin{array}{c}\text { Type } \\
\text { of } \\
\text { results }\end{array}$} & \multicolumn{10}{|c|}{ Reference task throughputs } \\
\hline & & \multicolumn{2}{|c|}{$\overline{T_{1}}$} & \multicolumn{2}{|c|}{$\overline{T_{2}}$} & \multicolumn{2}{|c|}{$\overline{T_{3}}$} & \multicolumn{2}{|c|}{$\overline{T_{4}}$} & \multicolumn{2}{|c|}{$\overline{T_{5}}$} \\
\hline & & thru & err\% & thru & err\% & thru & err\% & thru & err\% & thru & err\% \\
\hline \multirow[t]{2}{*}{$\overline{61}$} & exact & 0.0308 & & 0.0304 & & 0.0300 & & 0.0297 & & 0.0291 & \\
\hline & approx & 9.0308 & -0.13 & 0.0304 & -0.11 & 0.0300 & -0.09 & 0.0297 & -0.08 & 0.0291 & -0.06 \\
\hline \multirow[t]{2}{*}{62} & exact & $\overline{0.0485}$ & & 0.0415 & & 0.0363 & & 0.0323 & & $0.02 \overline{288}$ & \\
\hline & approx & 0.0483 & -0.38 & 0.0414 & -0.26 & 0.0363 & -0.19 & 0.0323 & -0.14 & 0. J288 & -0.10 \\
\hline \multirow[t]{2}{*}{63} & exact & 0.0593 & & 0.0507 & & 0.0461 & & 0.0393 & & 0.0384 & \\
\hline & approx & 0.0578 & -2.56 & 0.0505 & -0.38 & 0.0455 & -1.30 & 0.0390 & -0.91 & 0.0382 & -0.51 \\
\hline \multirow[t]{2}{*}{64} & exact & 0.0495 & & 0.0438 & & 0.0382 & & 0.0351 & & 0.0344 & \\
\hline & approx & 0.0479 & -3.18 & 0.0435 & -0.56 & 0.0381 & -0.31 & 0.0347 & -1.06 & 0.0343 & -0.54 \\
\hline \multirow[t]{2}{*}{65} & exact & 0.0273 & & 0.0279 & & 0.0257 & & 0.0240 & & 0.0238 & \\
\hline & approx & 0.0268 & -1.75 & 0.0278 & -0.65 & 0.0258 & 0.21 & 0.0237 & -1.16 & 0.0236 & -0.53 \\
\hline \multirow[t]{2}{*}{66} & exact & 0.0511 & & 0.0415 & & 0.0363 & & 0.0338 & & 0.0333 & \\
\hline & approx & 0.0511 & -0.10 & 0.0414 & -0.43 & 0.0362 & -0.16 & 0.0335 & -0.81 & 0.0332 & -0.37 \\
\hline \multirow[t]{2}{*}{67} & exact & 0.0576 & & 0.0453 & & 0.0390 & & 0.0347 & & 0.0255 & \\
\hline & approx & 0.0577 & 0.15 & 0.0450 & -0.71 & 0.0388 & -0.42 & 0.0344 & -1.03 & 0.0254 & -0.39 \\
\hline \multirow[t]{2}{*}{68} & exact & 0.0437 & & 0.0362 & & 0.0320 & & 0.0294 & & 0.0131 & \\
\hline & approx & 0.0438 & 0.15 & 0.0360 & -0.72 & 0.0318 & -0.45 & 0.0292 & -0.39 & 0.0130 & -0.23 \\
\hline \multirow[t]{2}{*}{69} & exact & 0.0324 & & 0.0282 & & 0.0253 & & 0.0236 & & 0.0105 & \\
\hline & approx & 0.0328 & 1.20 & 0.0280 & -0.90 & 0.0251 & -0.67 & 0.0234 & -0.50 & 0.0105 & -0.08 \\
\hline \multirow[t]{2}{*}{70} & exact & 0.0268 & & 0.0226 & & 0.0216 & & 0.0204 & & 0.0099 & \\
\hline & approx & 0.0265 & -1.14 & 0.0228 & 0.64 & 0.0214 & -0.87 & 0.0202 & -0.61 & 0.0099 & -0.08 \\
\hline \multirow[t]{2}{*}{71} & exact & 0.0240 & & 0.0208 & & 0.0199 & & 0.0188 & & 0.0102 & \\
\hline & approx & 0.0236 & -1.42 & 0.0210 & 0.77 & 0.0198 & -0.86 & 0.0187 & -0.70 & 0.0102 & -0.12 \\
\hline \multirow[t]{2}{*}{72} & exact & 0.0259 & & 0.0220 & & 0.0210 & & 0.0160 & & 0.0104 & \\
\hline & approx & 0.0257 & -0.72 & 0.0222 & 0.65 & 0.0208 & -1.19 & 0.0159 & -0.97 & 0.0104 & -0.22 \\
\hline \multirow[t]{2}{*}{73} & exact & 0.0286 & & 0.0195 & & 0.0223 & & 0.0166 & & 0.0106 & \\
\hline & approx & 0.0289 & 1.25 & 0.0193 & -0.70 & 0.0220 & -1.32 & 0.0164 & -1.17 & 0.0106 & -0.29 \\
\hline \multirow[t]{2}{*}{74} & exact & 0.1006 & & 0.0423 & & 0.0490 & & 0.0428 & & 0.0166 & \\
\hline & approx & 0.1010 & 0.39 & 0.0417 & -1.43 & 0.0484 & -1.10 & 0.0420 & -1.71 & 0.0165 & -0.34 \\
\hline 75 & exact & 0.0579 & & 0.0317 & & 0.0355 & & 0.0320 & & 0.0140 & \\
\hline & approx & 0.0600 & 3.53 & 0.0311 & -1.65 & 0.0351 & -1.04 & 0.0318 & -0.80 & 0.0139 & -0.39 \\
\hline 76 & exact & 0.0363 & & 0.0244 & & 0.0269 & & 0.0248 & & 0.0110 & \\
\hline & approx & 0.0368 & 1.28 & 0.0240 & -1.76 & 0.0267 & -0.97 & 0.0246 & -0.78 & 0.0110 & -0.15 \\
\hline 77 & exact & 0.0292 & & 0.0200 & & 0.0228 & & 0.0213 & & 0.0101 & \\
\hline & approx & 0.0295 & 1.03 & 0.0199 & -0.33 & 0.0225 & -1.07 & 0.0211 & -0.80 & 0.0100 & -0.12 \\
\hline 78 & exact & 0.0261 & & 0.0181 & & $0.0 \overline{205}$ & & 0.0172 & & 0.0116 & \\
\hline & approx & 0.0267 & 2.63 & 0.0180 & -0.52 & 0.0203 & -1.23 & 0.0170 & -1.08 & 0.0115 & -0.25 \\
\hline 79 & exact & 0.0310 & & 0.0248 & & 0.0239 & & 0.0223 & & 0.0137 & \\
\hline & approx & 0.0313 & 0.94 & 0.0244 & -1.53 & 0.0238 & -0.78 & 0.0222 & -0.50 & 0.0137 & -0.12 \\
\hline$\overline{80}$ & exact & 0.0531 & & 0.0500 & & 0.0500 & & 0.0518 & & 0.0534 & \\
\hline & approx & 0.0532 & 0.04 & 0.0500 & 0.08 & 0.0500 & 0.08 & 0.0516 & -0.28 & 0.0535 & 0.03 \\
\hline
\end{tabular}

continues on the next page 
CS5 results continued

\begin{tabular}{|c|c|c|c|c|c|c|c|c|c|c|c|}
\hline \multirow{3}{*}{ Case } & \multirow{3}{*}{$\begin{array}{l}\text { Type } \\
\text { of } \\
\text { results }\end{array}$} & \multicolumn{10}{|c|}{ Reference task throughputs } \\
\hline & & \multicolumn{2}{|c|}{$\overline{T_{1}}$} & \multicolumn{2}{|c|}{$T_{2}$} & \multicolumn{2}{|c|}{$T_{3}$} & \multicolumn{2}{|c|}{$\bar{T}_{4}$} & \multicolumn{2}{|c|}{$\bar{T}$} \\
\hline & & thru & exr\% & thru & err\% & thru & err\% & thru & err\% & thru & err\% \\
\hline \multirow[t]{2}{*}{81} & exact & 0.0258 & & 0.0252 & & 0.0252 & & 0.0256 & & 0.0259 & \\
\hline & rox & 0.1 & 0.24 & 52 & 0.20 & 0.0253 & 0.19 & .0256 & -0.11 & 0.0258 & -0.15 \\
\hline \multirow[t]{2}{*}{82} & $\overline{\operatorname{exact}}$ & 0.0272 & & 0.0264 & & 0.0265 & & 0.0222 & & 0.0246 & \\
\hline & prox & 0.0272 & 0.23 & 0.0264 & 0.13 & 65 & 0.03 & 0.0222 & -0.22 & 0.0245 & -0.23 \\
\hline \multirow[t]{2}{*}{83} & $a t$ & 0.0288 & & 0.0279 & & & & 0.0186 & & $0 . \overline{0225}$ & \\
\hline & brox & 288 & 0.28 & 0.0279 & 0.08 & & 0.01 & C0185 & -0.22 & 0.0224 & -0.35 \\
\hline \multirow[t]{2}{*}{84} & exact & 0.0311 & & 0.0300 & & $\overline{0.6}$ & & 0.0138 & & 0.0189 & \\
\hline & approx & 0.0312 & 0.39 & 0.0300 & -0.02 & 0. & -0.06 & 0.0137 & -0.15 & 0.0188 & -0.31 \\
\hline \multirow[t]{2}{*}{85} & exact & 0.0325 & & 0.0313 & & & & 0.0140 & & 0.0123 & \\
\hline & approx & 0.0326 & 0.37 & 0.0313 & -0.06 & 0.0314 & -0.09 & 0.0140 & -0.16 & 0.0128 & $-0.16)$ \\
\hline \multirow[t]{2}{*}{86} & exact & 0.0354 & & 0.0339 & & 0.0340 & & 0.0077 & & 0.0090 & \\
\hline & approx & 0.0354 & 0.22 & 0.0339 & -0.06 & 0.0340 & -0.09 & 0.0077 & -0.05 & 0.0090 & $-0.06)$ \\
\hline \multirow[t]{2}{*}{87} & exact & 0.0237 & & 0.0230 & & 0.0225 & & 0.0220 & & 0.0216 & \\
\hline & approx & 0.0234 & -1.20 & 0.0229 & -0.37 & 0. & 0.12 & 0.0221 & 0.22 & 0.0217 & .17 \\
\hline \multirow[t]{2}{*}{88} & exact & 0.0291 & & 0.0279 & & 0.0270 & & 0.0263 & & 0.0257 & \\
\hline & approx & 0.0284 & -2.29 & 0.0278 & -0.35 & 271 & 0.19 & 0.0264 & 31 & 0.0258 & 23 \\
\hline \multirow[t]{2}{*}{89} & exact & 0.0288 & & 0.0278 & & 70 & & 0.0264 & & 0.0258 & \\
\hline & approx & 0 & -0.98 & 0.0277 & -0.39 & & 0.10 & 0.0264 & .18 & 0.0259 & 14 \\
\hline \multirow[t]{2}{*}{90} & exact & 108 & & 0.0105 & & $\overline{01}$ & & 0.0098 & & 0.0097 & \\
\hline & approx & & -2.50 & 0. & -1.56 & & .47 & & 19 & 097 & .12 \\
\hline \multirow[t]{2}{*}{91} & exact & 0.0144 & & 0.0140 & & 0.0134 & & 0.0130 & & 0.0127 & \\
\hline & approx & 0.0144 & 0.47 & 0.0139 & -0.87 & & 0.01 & 0.0130 & 0.08 & 0.0127 & 0.0 .5 \\
\hline \multirow[t]{2}{*}{92} & exact & 0.0042 & & 0.0040 & & 0.0037 & & 0.0031 & & 0.0030 & \\
\hline & approx & 0.0043 & 2.30 & 0.0039 & -1.18 & 0.0036 & -1.20 & 0.0031 & -0.26 & 0.0029 & -1.39 \\
\hline \multirow[t]{2}{*}{93} & exact & 0.0045 & & 0.0040 & & 0.0040 & & 0.0032 & & 0.0032 & \\
\hline & approx & 0.0047 & 3.25 & 0.0040 & -0.72 & & 0.10 & 0.0032 & -0.12 & 0.0031 & $-1.3 x$ \\
\hline \multirow[t]{2}{*}{94} & exact & 0.0052 & & 0.0046 & & 0.0048 & & 0.0035 & & 0.0035 & \\
\hline & approx & & 3.75 & & 0.13 & & -0.56 & & -0.03 & 0.0035 & $j 9$ \\
\hline \multirow[t]{2}{*}{$\overline{95}$} & exact & 0.0040 & & 0.0038 & & 0.0039 & & 0.0031 & & 0.0030 & \\
\hline & approx & 0.0040 & 0.55 & 0.0038 & -0.53 & 0.0039 & -0.43 & 0.0031 & 0.00 & 0.0030 & -0.72 \\
\hline
\end{tabular}


Table B.7: Exact and approx. results for test suite MR3 (mean error $0.38 \%$ )

\begin{tabular}{|c|c|c|c|c|c|c|c|}
\hline \multirow{3}{*}{ Case } & \multirow{3}{*}{$\begin{array}{l}\text { Type } \\
\text { of } \\
\text { results }\end{array}$} & \multicolumn{6}{|c|}{ Reference task throughputs } \\
\hline & & \multicolumn{2}{|c|}{$\overline{T_{1}}$} & \multicolumn{2}{|c|}{$T_{2}$} & \multicolumn{2}{|c|}{$\overline{T_{3}}$} \\
\hline & & thru & err[\%] & thru & err[\%] & thru & $\overline{\operatorname{err}[}$ \\
\hline \multirow[t]{2}{*}{$\bar{I}$} & exact & 0.1117 & & 0.1484 & & 0.1310 & \\
\hline & prox & 0.1114 & -0.30 & 0.1487 & 0.21 & 1300 & 0.72 \\
\hline \multirow[t]{2}{*}{$\overline{2}$} & act & 0.1920 & & 0.2133 & & 0.2196 & \\
\hline & rox & 16 & -0.19 & .2130 & -0.15 & 2198 & .10 \\
\hline \multirow[t]{2}{*}{$\overline{3}$} & act & 0.1574 & & 0.0800 & & $\overline{0.0765}$ & \\
\hline & prox & 57 & -1.06 & 0.0790 & -1.16 & 0.0762 & -0.30 \\
\hline \multirow[t]{2}{*}{4} & act & $\overline{0.0726}$ & & 0.0378 & & $\overline{0.0797}$ & \\
\hline & prox & 0.0721 & -0.67 & 0.0377 & .0 .09 & .0803 & 1.79 \\
\hline & act & 0.0561 & & 0.0571 & & 0.0589 & \\
\hline & prox & 0 & .28 & .0570 & .22 & .0590 & 15 \\
\hline \multirow[t]{2}{*}{$\overline{6}$} & act & $\overline{0}$. & & 0.0499 & & $\overline{0773}$ & \\
\hline & prox & 4 & -0.65 & 0.0498 & -0.13 & 0.0778 & .70 \\
\hline \multirow[t]{2}{*}{7} & act & $\overline{527}$ & & 0.2473 & & $0 . \overline{1937}$ & \\
\hline & rox & 491 & -1.02 & 0.2454 & -0.78 & 0.1 & -0.60 \\
\hline \multirow[t]{2}{*}{8} & act & 0.3860 & & 0.2607 & & 0.2012 & \\
\hline & rox & & 0.67 & .2590 & -0.64 & 0.2001 & 0.51 \\
\hline \multirow[t]{2}{*}{9} & act & 0.0 & & 0.2058 & & 0.1727 & \\
\hline & rox & & 07 & 0.2057 & .05 & 0.1717 & 0.56 \\
\hline \multirow[t]{2}{*}{10} & act & 0.0094 & & 0.2618 & & 0.2141 & \\
\hline & prox & & 00 & 2617 & -0.02 & 2140 & 1.07 \\
\hline \multirow[t]{2}{*}{11} & act & & & 0.1741 & & 0.1563 & \\
\hline & rox & & 14 & .1735 & -0.31 & 0.1561 & 0.08 \\
\hline \multirow[t]{2}{*}{12} & act & 0.1679 & & 0.1379 & & 0.1965 & \\
\hline & prox & 71 & 0.44 & 0.1375 & -0.25 & 0.1963 & -0.07 \\
\hline \multirow[t]{2}{*}{13} & exact & 0.1140 & & 0.0924 & & 0.1146 & \\
\hline & approx & 140 & -0.05 & 0.0923 & -0.10 & 0.1146 & 0.05 \\
\hline \multirow[t]{2}{*}{14} & exact & 0.1194 & & 0.1131 & & 0.1273 & \\
\hline & rox & & -0.13 & & .37 & & 000 \\
\hline \multirow[t]{2}{*}{15} & act & 0.2028 & & 0.1594 & & 0.1782 & \\
\hline & & & 09 & & -0.30 & & -0.66 \\
\hline \multirow[t]{2}{*}{16} & exact & 0.0781 & & 0.0995 & & 0.0953 & \\
\hline & rox & 779 & -0.21 & 0.0998 & 0.28 & 0.0942 & -1.08 \\
\hline \multirow[t]{2}{*}{17} & exact & 0.1803 & & 0.2647 & & 0.2368 & \\
\hline & rox & 795 & -0.42 & 0.2647 & 0.00 & 0.2359 & 0 \\
\hline
\end{tabular}

continues on the next page 
Table B.7 continued: MR3 results

\begin{tabular}{|c|c|c|c|c|c|c|c|}
\hline \multirow{3}{*}{ Case } & \multirow{3}{*}{$\begin{array}{l}\text { Type } \\
\text { of } \\
\text { results }\end{array}$} & \multicolumn{6}{|c|}{ Reference task throughputs } \\
\hline & & \multicolumn{2}{|c|}{$T_{1}$} & \multicolumn{2}{|c|}{$T_{2}$} & \multicolumn{2}{|c|}{$\overline{T_{3}}$} \\
\hline & & thru & err[\%] & thru & err[\%] & thru & err[\%] \\
\hline \multirow[t]{2}{*}{18} & exact & 0.0914 & & 0.1085 & & 0.1031 & \\
\hline & approx & 0.0911 & -0.35 & 0.1089 & 0.42 & 0.1026 & -0.51 \\
\hline \multirow[t]{2}{*}{19} & exact & $\overline{0.0913}$ & & 0.1089 & & 0.1136 & \\
\hline & approx & 0.0911 & -0.15 & 0.1085 & -0.34 & 0.1144 & 0.77 \\
\hline \multirow[t]{2}{*}{20} & exact & 0.0939 & & 0.1125 & & 0.1100 & \\
\hline & approx & 0.0937 & -0.26 & 0.1128 & 0.25 & 0.1096 & -0.34 \\
\hline \multirow[t]{2}{*}{$\overline{21}$} & exact & 0.1828 & & 0.2922 & & 0.2880 & \\
\hline & approx & 0.1826 & -0.10 & 0.2922 & -0.01 & 0.2880 & 0.02 \\
\hline \multirow[t]{2}{*}{22} & exact & 0.2022 & & 0.3756 & & 0.2510 & \\
\hline & approx & 0.2015 & -0.34 & 0.3766 & 0.29 & 0.2481 & -1.17 \\
\hline \multirow[t]{2}{*}{23} & exact & $\overline{0.1894}$ & & 0.3175 & & 0.2841 & \\
\hline & approx & 0.1891 & -0.19 & 0.3184 & 0.26 & 0.2827 & -0.49 \\
\hline \multirow[t]{2}{*}{24} & exact & 0.1851 & & 0.3193 & & 0.2154 & \\
\hline & approx & 0.1843 & $-\overbrace . 4 1$ & 0.3206 & 0.42 & 0.2133 & .0 .96 \\
\hline \multirow[t]{2}{*}{25} & exact & 0.1712 & & 0.2450 & & 0.1989 & \\
\hline & approx & 0.1705 & .0 .40 & 0.2451 & 0.07 & 0.1970 & -0.91 \\
\hline \multirow[t]{2}{*}{26} & exact & 0.1270 & & $0.1 \mathrm{f}(\overline{6}$ & & 0.1384 & \\
\hline & approx & 0.1266 & -0.31 & 0.1651 & 0.30 & 0.1374 & -0.76 \\
\hline \multirow[t]{2}{*}{$2 ?$} & exact & 0.1080 & & 0.1348 & & 0.1228 & \\
\hline & approx & 0.1078 & -0.17 & 0.1353 & 0.33 & 0.1217 & -0.97 \\
\hline \multirow[t]{2}{*}{28} & exact & 0.1377 & & 0.1493 & & 0.1328 & \\
\hline & approx & 0.1375 & -0.15 & 0.1494 & 0.06 & 0.1327 & -0.06 \\
\hline \multirow{2}{*}{29} & exact & 0.2565 & & 0.2565 & & 0.2129 & \\
\hline & approx & 0.2560 & -0.21 & 0.2560 & -0.21 & 0.2128 & .0 .04 \\
\hline \multirow[t]{2}{*}{30} & exact & 0.2087 & & 0.2073 & & 0.1852 & \\
\hline & approx & 0.2090 & -0.33 & 0.2072 & -0.05 & 0.1843 & -0.48 \\
\hline \multirow[t]{2}{*}{31} & exact & 0.3131 & & 0.2800 & & 0.2391 & \\
\hline & approx & 0.3139 & 0.26 & 0.2786 & -0.50 & 0.2383 & -0.35 \\
\hline \multirow[t]{2}{*}{$\overline{32}$} & exact & 0.2070 & & 0.3356 & & 0.2746 & \\
\hline & approx & 0.2058 & -0.56 & 0.3336 & -0.59 & 0.2724 & -0.81 \\
\hline \multirow[t]{2}{*}{$\overline{33}$} & exact & 0.2091 & & 0.2737 & & 0.2355 & \\
\hline & approx & 0.2081 & -0.49 & 0.2736 & -0.05 & 0.2337 & -0.76 \\
\hline \multirow[t]{2}{*}{34} & exact & 0.0786 & & 0.1178 & & 0.1082 & \\
\hline & approx & 0.0785 & -0.18 & 0.1182 & 0.31 & 0.1071 & -1.02 \\
\hline \multirow[t]{2}{*}{$\overline{35}$} & exact & 0.1043 & & 0.1835 & & 0.1676 & \\
\hline & approx & 0.1041 & -0.23 & 0.1840 & 0.27 & 0.1659 & -1.01 \\
\hline
\end{tabular}


Table B.8: Exact and approx. results for test suite MR4 (mean error 0.47\%)

\begin{tabular}{|c|c|c|c|c|c|c|c|c|c|}
\hline \multirow{3}{*}{ Case } & \multirow{3}{*}{$\begin{array}{c}\text { Type } \\
\text { of } \\
\text { results }\end{array}$} & \multicolumn{8}{|c|}{ Reference task throughputs } \\
\hline & & \multicolumn{2}{|c|}{$\overline{T_{1}}$} & \multicolumn{2}{|c|}{$\overline{T_{2}}$} & \multicolumn{2}{|c|}{$T_{3}$} & \multicolumn{2}{|c|}{$\overline{T_{4}}$} \\
\hline & & thru & err[\%] & thru & $\operatorname{err}[\%]$ & thru & err[\%] & thru & $\operatorname{err}[\%]$ \\
\hline \multirow[t]{2}{*}{1} & exact & 0.0833 & & 0.0959 & & 0.0892 & & 0.0885 & \\
\hline & approx & 0.0831 & -0.20 & 0.0961 & 0.21 & 0.0888 & -0.36 & 0.0886 & 0.01 \\
\hline \multirow[t]{2}{*}{$\overline{2}$} & exact & 0.0907 & & 0.1073 & & 0.0974 & & 0.1017 & \\
\hline & approx & 0.0906 & -0.19 & 0.1075 & 0.10 & 0.0968 & -0.57 & 0.1020 & 0.31 \\
\hline \multirow[t]{2}{*}{3} & exact & 0.0623 & & 0.0959 & & 0.0943 & & 0.0920 & \\
\hline & approx & 0.0618 & -0.78 & 0.0943 & -1.65 & 0.0926 & -1.80 & 0.0907 & -1.42 \\
\hline \multirow[t]{2}{*}{4} & exact & 0.2037 & & 0.2037 & & 0.2037 & & 0.2037 & \\
\hline & approx & 0.2013 & -1.17 & 0.2013 & -1.17 & 0.2013 & -1.17 & 0.2013 & -1.17 \\
\hline \multirow[t]{2}{*}{5} & exact & $\longdiv { 0 . 2 3 7 2 }$ & & 0.2372 & & 0.2347 & & 0.4030 & \\
\hline & approx & 0.2345 & -1.15 & 0.2345 & -1.15 & 0.2318 & -1.24 & 0.4007 & -0.57 \\
\hline \multirow[t]{2}{*}{6} & exact & 0.0614 & & 0.0571 & & 0.0545 & & 0.0519 & \\
\hline & approx & 0.0616 & 0.29 & 0.0570 & -0.27 & 0.0542 & -0.43 & 0.0517 & -0.42 \\
\hline \multirow[t]{2}{*}{$\overline{7}$} & exact & $\overline{0.1352}$ & & 0.1375 & & 0.1660 & & 0.1621 & \\
\hline & approx & 0.1347 & -0.39 & 0.1368 & -0.51 & 0.1651 & -0.50 & 0.1623 & 0.09 \\
\hline \multirow[t]{2}{*}{8} & exact & 0.1273 & & 0.1299 & & 0.1318 & & 0.1272 & \\
\hline & approx & 0.1260 & -1.04 & 0.1284 & -1.11 & 0.1303 & -1.13 & 0.1261 & -0.92 \\
\hline \multirow[t]{2}{*}{9} & exact & 0.1432 & & 0.1892 & & 0.1737 & & 0.2236 & \\
\hline & approx & 0.1430 & -0.20 & 0.1889 & -0.16 & 0.1728 & -0.54 & 0.2253 & 0.79 \\
\hline \multirow[t]{2}{*}{10} & exact & 0.1280 & & 0.1255 & & 0.1154 & & 0.1021 & \\
\hline & approx & 0.1289 & 0.71 & 0.1250 & -0.36 & 0.1149 & -0.42 & 0.1012 & -0.90 \\
\hline \multirow[t]{2}{*}{11} & exact & 0.1172 & & 0.1141 & & 0.1063 & & 0.0963 & \\
\hline & approx & 0.1178 & 0.47 & 0.1138 & -0.22 & 0.1061 & -0.24 & 0.0961 & -0.15 \\
\hline \multirow[t]{2}{*}{12} & exact & 0.1142 & & 0.0991 & & 0.1245 & & 0.1171 & \\
\hline & approx & 0.1137 & -0.43 & 0.0989 & -0.21 & 0.1252 & 0.55 & 0.1167 & -0.33 \\
\hline \multirow[t]{2}{*}{13} & exact & 0.0803 & & 0.0694 & & 0.0796 & & 0.0762 & \\
\hline & approx & 0.0801 & -0.29 & 0.0693 & -0.06 & 0.0802 & 0.71 & 0.0762 & -0.08 \\
\hline \multirow[t]{2}{*}{14} & exact & $\mathbf{0 . 0 7 8 1}$ & & 0.0748 & & 0.0793 & & 0.0759 & \\
\hline & .pprox & 0.0783 & 0.24 & 0.0746 & -0.28 & 0.0792 & -0.10 & 0.0758 & -0.17 \\
\hline \multirow[t]{2}{*}{15} & exact & 0.1497 & & 0.1256 & & 0.1347 & & 0.1290 & \\
\hline & approx & 0.1499 & 0.17 & 0.1253 & -0.22 & 0.1342 & -0.42 & 0.1287 & -0.20 \\
\hline \multirow[t]{2}{*}{16} & exact & 0.0903 & & 0.1066 & & 0.1035 & & 0.1000 & \\
\hline & approx & 0.0902 & -0.12 & 0.1069 & 0.31 & 0.1029 & -0.56 & 0.0998 & -0.17 \\
\hline \multirow[t]{2}{*}{17} & exact & 0.1292 & & 0.1601 & & 0.1490 & & 0.1426 & \\
\hline & approx & 0.1286 & -0.46 & 0.1601 & -0.02 & 0.1490 & -0.02 & 0.1428 & 0.10 \\
\hline
\end{tabular}

continues on the next page 
Table B.8 continued: MR4 results

\begin{tabular}{|c|c|c|c|c|c|c|c|c|c|}
\hline \multirow{3}{*}{ Case } & \multirow{3}{*}{$\begin{array}{c}\text { Type } \\
\text { of } \\
\text { results }\end{array}$} & \multicolumn{8}{|c|}{ Reference task throughputs } \\
\hline & & \multicolumn{2}{|c|}{$T_{1}$} & \multicolumn{2}{|c|}{$T_{2}$} & \multicolumn{2}{|c|}{$T_{3}$} & \multicolumn{2}{|c|}{$T_{t}$} \\
\hline & & thru & err[\%] & thru & err[\%] & thru & $\operatorname{err}[\%]$ & thru & $\operatorname{err}[\cdots]$ \\
\hline \multirow[t]{2}{*}{18} & exact & $\widehat{0.0862}$ & & 0.0998 & & 0.0956 & & 0.0924 & \\
\hline & approx & 0.0857 & -0.60 & 0.1003 & 0.50 & 0.0954 & -0.25 & 0.0921 & .0 .26 \\
\hline \multirow[t]{2}{*}{19} & exact & 0.0909 & & 0.1072 & & 0.1098 & & 0.0956 & \\
\hline & approx & 0.0909 & 0.08 & 0.1078 & 0.62 & 0.1090 & -0.76 & 0.0959 & 0.37 \\
\hline \multirow[t]{2}{*}{20} & exact & 0.0926 & & 0.1103 & & 0.1078 & & 0.0987 & \\
\hline & approx & 0.0921 & -0.62 & 0.1107 & 0.37 & 0.1078 & -0.09 & 0.0983 & -0.10 \\
\hline \multirow[t]{2}{*}{21} & exact & 0.1423 & & 0.1933 & & 0.1901 & & 0.1563 & \\
\hline & approx & 0.1420 & -0.16 & 0.1926 & -0.39 & 0.1917 & 0.82 & 0.1560 & -0.17 \\
\hline \multirow[t]{2}{*}{22} & exact & 0.1539 & & 0.2262 & & 0.1744 & & 0.1708 & \\
\hline & approx & 0.1527 & -0.74 & 0.2283 & 0.94 & 0.1729 & .0 .89 & 0.1702 & -0.3 .1 \\
\hline \multirow[t]{2}{*}{23} & exact & 0.1459 & & 0.2032 & & 0.1880 & & 0.1609 & \\
\hline & approx & 0.1454 & -0.36 & 0.2040 & 0.40 & 0.1878 & -0.07 & 0.1606 & -0.21 \\
\hline \multirow[t]{2}{*}{24} & exact & 0.1413 & & 0.1977 & & 0.1542 & & 0.157 .4 & \\
\hline & -approx & 0.1406 & -0.52 & 0.1993 & 0.81 & 0.1531 & -0.74 & 0.1570 & -0.28 \\
\hline \multirow[t]{2}{*}{25} & exact & 0.1356 & & 0.1706 & & 0.1466 & & 0.1503 & \\
\hline & approx & 0.1348 & -0.58 & 0.1713 & 0.44 & 0.1458 & -0.57 & 0.1501 & .0 .16 \\
\hline \multirow[t]{2}{*}{26} & exact & 0.1123 & & 0.1390 & & 0.1213 & & 0.1224 & \\
\hline & approx & 0.1117 & -0.50 & 0.1397 & 0.47 & 0.1207 & -0.55 & 0.1222 & -0.17 \\
\hline \multirow[t]{2}{*}{27} & exact & 0.0933 & & 0.1118 & & 0.1046 & & 0.1026 & \\
\hline & approx & 0.0931 & -0.26 & 0.1125 & 0.67 & 0.1036 & -0.92 & 0.1021 & -0.17 \\
\hline \multirow[t]{2}{*}{28} & exact & 0.1177 & & 0.1264 & & 0.1152 & & 0.1121 & \\
\hline & approx & 0.1177 & -0.07 & 0.1262 & -0.19 & 0.1153 & 0.11 & 0.1123 & 0.16 \\
\hline \multirow[t]{2}{*}{29} & exact & 0.1866 & & 0.1885 & & 0.1648 & & 0.1594 & \\
\hline & approx & 0.1863 & -0.14 & 0.1873 & -0.65 & 0.1651 & 0.18 & 0.1597 & 0.22 \\
\hline \multirow[t]{2}{*}{30} & exact & 0.1735 & & 0.1706 & & 0.1566 & & 0.1602 & \\
\hline & approx & 0.1728 & -0.40 & 0.1712 & 0.36 & 0.1561 & -0.30 & 0.1595 & -0.11 \\
\hline \multirow[t]{2}{*}{31} & exact & 0.2147 & & 0.2002 & & 0.1802 & & 0.1834 & \\
\hline & approx & 0.2160 & 0.61 & 0.1996 & $-0,30$ & 0.1795 & -0.37 & 0.1828 & .0 .32 \\
\hline \multirow[t]{2}{*}{32} & exact & 0.1586 & & 0.2203 & & 0.1351 & & 0.1986 & \\
\hline & approx & 0.1578 & .0 .54 & 0.2203 & -0.02 & 0.1939 & -0.60 & 0.1976 & .0 .51 \\
\hline \multirow[t]{2}{*}{$\overline{33}$} & exact & 0.1589 & & 0.1852 & & 0.1660 & & 0.1649 & \\
\hline & approx & 0.1580 & -0.59 & 0.1857 & 0.26 & 0.1654 & -0.36 & 0.1648 & -0.07 \\
\hline \multirow[t]{2}{*}{$\overline{34}$} & exact & 0.0693 & & 0.0935 & & 0.0872 & & 0.0865 & \\
\hline & approx & 0.0691 & -0.32 & 0.0941 & 0.70 & 0.0865 & -0.79 & 0.0859 & -0.7 .1 \\
\hline \multirow[t]{2}{*}{35} & exact & 0.0956 & & 0.1496 & & 0.1392 & & 0.1378 & \\
\hline & approx & 0.0952 & -0.36 & 0.1507 & 0.71 & 0.1382 & -0.77 & 0.1369 & -0.60 \\
\hline
\end{tabular}


Table B.9: Exact and approximate results for test suite MR5 (mean error 0.63\%)

\begin{tabular}{|c|c|c|c|c|c|c|c|c|c|c|c|}
\hline \multirow{3}{*}{ Case } & \multirow{3}{*}{$\begin{array}{l}\text { Type } \\
\text { of } \\
\text { results }\end{array}$} & \multicolumn{10}{|c|}{ Reference task throughputs } \\
\hline & & \multicolumn{2}{|c|}{$\overline{T_{1}}$} & \multicolumn{2}{|c|}{$\overline{T_{2}}$} & \multicolumn{2}{|c|}{$T_{3}$} & \multicolumn{2}{|c|}{$\bar{T}$} & \multicolumn{2}{|c|}{$T_{\mathbf{5}}$} \\
\hline & & thru & $r \%$ & thru & err\% & thru & $\mathrm{rr} \%$ & thru & err\% & thru & err\% \\
\hline I & $\begin{array}{l}\text { exact } \\
\text { approx }\end{array}$ & $\begin{array}{l}0.0875 \\
0.0873\end{array}$ & -0.19 & $\begin{array}{l}0.0999 \\
0.0998\end{array}$ & -0.19 & $\begin{array}{l}0.0947 \\
0.0946\end{array}$ & -0.17 & $\begin{array}{l}0.0937 \\
0.0939\end{array}$ & 0.23 & $\begin{array}{l}0.0937 \\
0.0939\end{array}$ & 0.23 \\
\hline$\overline{2}$ & $\begin{array}{l}\text { exact } \\
\text { approx }\end{array}$ & $\begin{array}{l}0.0993 \\
0.0991\end{array}$ & -0.18 & $\begin{array}{l}0.1157 \\
0.1163\end{array}$ & -0.33 & $\begin{array}{l}0.1080 \\
0.1077\end{array}$ & -0.33 & $\begin{array}{l}0.1119 \\
0.1124\end{array}$ & 0.39 & $\begin{array}{l}0.1134 \\
0.1140\end{array}$ & 0.54 \\
\hline 3 & $\begin{array}{l}\text { exact } \\
\text { approx }\end{array}$ & $\begin{array}{l}0.0547 \\
0.0543\end{array}$ & -0.83 & $\begin{array}{l}0.0757 \\
0.0742\end{array}$ & -1.98 & $\begin{array}{l}0.0762 \\
0.0749\end{array}$ & .72 & $\begin{array}{l}0.0748 \\
0.0738 \\
\end{array}$ & 1.40 & $\begin{array}{l}0.1020 \\
0.1017 \\
\end{array}$ & 0.31 \\
\hline 4 & $\begin{array}{c}\text { exart } \\
\text { approx }\end{array}$ & $\begin{array}{l}0.0968 \\
0.0969\end{array}$ & 0.08 & $\begin{array}{l}0.0826 \\
0.0817\end{array}$ & -1.17 & $\begin{array}{l}0.0753 \\
0.0745\end{array}$ & -1.00 & $\begin{array}{l}0.0679 \\
0.0675\end{array}$ & -0.64 & $\begin{array}{l}0.0627 \\
0.0626\end{array}$ & 0.26 \\
\hline 5 & $\begin{array}{l}\text { :xact } \\
\text { approx }\end{array}$ & $\begin{array}{l}0.0528 \\
0.0533\end{array}$ & 0.88 & $\begin{array}{l}0.0502 \\
0.0502\end{array}$ & 0.08 & $\begin{array}{l}0.0480 \\
0.0479\end{array}$ & -0.21 & $\begin{array}{l}0.0460 \\
0.0458\end{array}$ & -0.30 & $\begin{array}{l}0.0443 \\
0.0441\end{array}$ & -0.30 \\
\hline$\overline{6}$ & $\begin{array}{c}\text { exact } \\
\text { approx }\end{array}$ & $\begin{array}{l}0.0585 \\
0.0588\end{array}$ & 0.55 & $\begin{array}{l}0.0550 \\
0.0549\end{array}$ & -0.26 & $\begin{array}{l}0.0512 \\
0.0510\end{array}$ & -0.31 & $\begin{array}{l}0.0477 \\
0.0475\end{array}$ & -0.33 & $\begin{array}{l}0.0442 \\
0.0440\end{array}$ & -0.39 \\
\hline 7 & $\begin{array}{l}\text { exact } \\
\text { approx }\end{array}$ & $\begin{array}{l}0.0339 \\
0.0335\end{array}$ & -1.07 & & -0.21 & $\begin{array}{l}0.0314 \\
0.0315\end{array}$ & 0.14 & $\begin{array}{l}0.0305 \\
0.0306\end{array}$ & 0.21 & $\begin{array}{l}0.0297 \\
0.0297\end{array}$ & .17 \\
\hline 8 & $\begin{array}{c}\text { exact } \\
\text { approx }\end{array}$ & $\begin{array}{l}0.0561 \\
0.0557\end{array}$ & .71 & & 0 & $\begin{array}{l}0.0481 \\
0.0481\end{array}$ & .02 & $\begin{array}{l}0.0452 \\
0.0453 \\
\end{array}$ & 13 & $\begin{array}{l}0.1426 \\
0.0427\end{array}$ & 12 \\
\hline$\overline{9}$ & $\begin{array}{l}\text { exact } \\
\text { approx }\end{array}$ & & -0.23 & & -0.37 & & -0.15 & & .49 & $\begin{array}{l}0.1274 \\
0.1274\end{array}$ & .02 \\
\hline 10 & $\begin{array}{c}\text { exact } \\
\text { approx }\end{array}$ & $\begin{array}{l}0.1154 \\
0.1134\end{array}$ & -1.73 & $\begin{array}{l}0.1190 \\
0.1168\end{array}$ & -1.92 & $\begin{array}{l}0.1178 \\
0.1156\end{array}$ & -1.85 & $\begin{array}{l}0.1131 \\
0.1112\end{array}$ & -1.63 & $\begin{array}{l}0.1146 \\
0.1129\end{array}$ & 1.52 \\
\hline 11 & $\begin{array}{l}\text { exact } \\
\text { approx }\end{array}$ & $\begin{array}{l}0.0808 \\
0.0812\end{array}$ & .45 & $\begin{array}{l}0.0799 \\
0.0798\end{array}$ & .08 & $\begin{array}{l}0.0761 \\
0.0761\end{array}$ & .03 & $\begin{array}{l}0.0699 \\
0.0694\end{array}$ & $70^{\circ}$ & $\begin{array}{l}0.0770 \\
0.0769\end{array}$ & .19 \\
\hline 12 & $\begin{array}{c}\text { exact } \\
\text { approx }\end{array}$ & & 01 & & .44 & & 19 & $\begin{array}{l}0.0676 \\
0.0676\end{array}$ & -0.01 & $\begin{array}{l}0.0728 \\
0.0729 \\
\end{array}$ & 8 \\
\hline 13 & $\begin{array}{l}\text { exact } \\
\text { approx }\end{array}$ & $\begin{array}{l}0.1208 \\
0.1209\end{array}$ & 0.06 & & 0.01 & & .49 & & 0.71 & & .70 \\
\hline$\overline{14}$ & $\begin{array}{l}\text { exact } \\
\text { approx }\end{array}$ & $\begin{array}{l}0.0993 \\
0.0995\end{array}$ & 18 & $\begin{array}{l}0.0826 \\
0.0826 \\
\end{array}$ & 0.04 & $\begin{array}{l}0.0999 \\
0.1001 \\
\end{array}$ & 18 & $\begin{array}{l}0.0935 \\
0.0930 \\
\end{array}$ & 0.48 & $\begin{array}{l}0.0887 \\
0.0883 \\
\end{array}$ & 0.45 \\
\hline 15 & $\begin{array}{c}\text { exact } \\
\text { approx }\end{array}$ & $\begin{array}{l}0.0996 \\
0.0997\end{array}$ & 6 & $\begin{array}{l}0.0956 \\
0.0954\end{array}$ & -0.20 & $\begin{array}{l}0.1030 \\
0.1034\end{array}$ & 39 & $\begin{array}{l}0.0963 \\
0.0958\end{array}$ & -0.0 & $\begin{array}{l}0.0914 \\
0.0909\end{array}$ & 0.64 \\
\hline 16 & $\begin{array}{l}\text { exact } \\
\text { approx }\end{array}$ & $\begin{array}{l}0.1372 \\
0.1385\end{array}$ & .95 & $\begin{array}{l}0.1205 \\
0.1200\end{array}$ & -0.41 & $\begin{array}{l}0.1296 \\
0.1289\end{array}$ & -0.49 & $\begin{array}{l}0.1278 \\
0.1264\end{array}$ & -1.12 & $\begin{array}{l}0.1205 \\
0.1200\end{array}$ & -0.41 \\
\hline 17 & $\begin{array}{c}\text { exact } \\
\text { approx }\end{array}$ & $\begin{array}{l}0.1387 \\
0.1396\end{array}$ & 62 & $\begin{array}{l}0.1209 \\
0.1205\end{array}$ & -0.37 & $\begin{array}{l}0.1292 \\
0.1287\end{array}$ & -0.36 & $\begin{array}{l}0.1234 \\
0.1229\end{array}$ & -0.43 & $\begin{array}{l}0.1209 \\
0.1205\end{array}$ & -0.37 \\
\hline 18 & $\begin{array}{l}\text { exact } \\
\text { approx }\end{array}$ & $\begin{array}{l}0.2138 \\
0.2115\end{array}$ & -1.09 & $\begin{array}{l}0.2138 \\
0.2115\end{array}$ & -1.09 & $\begin{array}{l}0.2114 \\
0.2089\end{array}$ & -1.17 & $\begin{array}{l}0.3276 \\
0.3269\end{array}$ & -0.23 & $\begin{array}{l}0.3276 \\
0.3269\end{array}$ & -0.23 \\
\hline 19 & $\begin{array}{l}\text { exact } \\
\text { approx }\end{array}$ & $\begin{array}{l}0.1659 \\
0.1643\end{array}$ & 97 & $\begin{array}{l}0.1659 \\
0.1643\end{array}$ & -0.97 & $\begin{array}{l}0.1659 \\
0.1643\end{array}$ & -0.97 & $\begin{array}{l}0.1659 \\
0.1643\end{array}$ & -0.97 & $\begin{array}{l}0.1659 \\
0.1643\end{array}$ & -0.97 \\
\hline 20 & $\begin{array}{c}\text { exact } \\
\text { approx }\end{array}$ & $\begin{array}{l}0.1000 \\
0.1000\end{array}$ & 0.00 & $\begin{array}{l}0.1000 \\
0.1000\end{array}$ & 0.00 & $\begin{array}{l}0.1000 \\
0.1000\end{array}$ & 0.00 & $\begin{array}{l}0.1000 \\
0.1000\end{array}$ & 0.00 & $\begin{array}{l}0.1000 \\
0.1000\end{array}$ & 0.00 \\
\hline
\end{tabular}

continues on the next page 
MR5 results continued

\begin{tabular}{|c|c|c|c|c|c|c|c|c|c|c|c|}
\hline \multirow{3}{*}{ Case } & \multirow{3}{*}{$\begin{array}{l}\text { Type } \\
\text { of } \\
\text { results }\end{array}$} & \multicolumn{10}{|c|}{ Reference task throughputs } \\
\hline & & \multicolumn{2}{|c|}{$T_{1}$} & \multicolumn{2}{|c|}{$\overline{T_{2}}$} & \multicolumn{2}{|c|}{$T_{3}$} & \multicolumn{2}{|c|}{$\overline{T_{4}}$} & \multicolumn{2}{|c|}{$T_{3}$} \\
\hline & & thru & err\% & thru & ert\% & thru & err\% & thru & err $\%$ & thiru & $\operatorname{rrr}^{i} i$ \\
\hline \multirow[t]{2}{*}{$\overline{21}$} & exact & 0.1030 & & 0.1313 & & 0.1161 & & 0.1098 & & $0.12 \cdot 11$ & \\
\hline & prox & 0.1030 & -0.06 & 0.1316 & 0.19 & 0.1158 & -0.26 & 0.1098 & 0.05 & 1239 & -11018.6 \\
\hline \multirow[t]{2}{*}{$\overline{22}$} & exact & 0.1170 & & 0.1514 & & 0.1324 & & 0.1244 & & 0.142 .4 & \\
\hline & prox & 0.1164 & -0.43 & 0.1516 & 0.13 & 0.1322 & -0.16 & 0.12 .44 & $0.0 i$ & 0.1426 & olti \\
\hline \multirow[t]{2}{*}{23} & exact & 0.1253 & & 0.1691 & & 0.1434 & & 0.1336 & & 0.155 .9 & \\
\hline & approx & 0.1244 & $-0.6 \%$ & 0.1687 & -0.25 & 0.1431 & -0.22 & 0.1335 & -0.05 & $0.15+60$ & 0.37 \\
\hline \multirow[t]{2}{*}{$\overline{24}$} & exact & 0.1074 & & 0.1408 & & 0.1240 & & 0.1167 & & 0.1330 & \\
\hline & approx & 0.1073 & -0.09 & 0.1409 & 0.05 & 0.1237 & -0.29 & 0.1167 & 0.03 & 0.1331 & 011 \\
\hline \multirow[t]{2}{*}{25} & exact & 0.1075 & & 0.1370 & & 0.1232 & & 0.1163 & & 0.1317 & \\
\hline & approx & 0.1072 & -0.25 & 0.1378 & 0.53 & 0.1228 & -0.31 & 0.1163 & $-0.0 \cdot 1$ & 01315 & -11.11 \\
\hline \multirow[t]{2}{*}{26} & exact & 0.1033 & & 0.1211 & & 0.1191 & & 0.1121 & & 0.127 & \\
\hline & prox & 0.1032 & .0 .07 & 0.1207 & $-\mathbf{0 . 3 3}$ & 1186 & -0.39 & 0.1122 & 0.06 & $0.12 \pi .1$ & 20 \\
\hline \multirow[t]{2}{*}{27} & exact & 0.1062 & & 0.1221 & & 76 & & 108 & & 62 & \\
\hline & approx & 0.1059 & -0.29 & 16 & -0.38 & & -0.12 & 108 & 0.05 & 267 & 12 \\
\hline \multirow[t]{2}{*}{28} & exact & 0.1016 & & 0.1153 & & 0.1229 & & 0.1189 & & 0.1191 & \\
\hline & approx & 0.1015 & -0.13 & 0.1150 & -0.26 & 31 & 0.17 & 0.1192 & 0.24 & 0.1191 & 0.03 \\
\hline \multirow[t]{2}{*}{29} & exact & 0.1434 & & 0.1767 & & 0.1991 & & 0.1886 & & $0.18 \times 2$ & \\
\hline & approx & 0.1430 & -0.28 & 0.1755 & -0.68 & 995 & 0.23 & 0.1889 & 0.19 & 0.1878 & .0 .18 \\
\hline \multirow[t]{2}{*}{30} & exact & 0.1036 & & 0.1199 & & 0.1287 & & 0.1243 & & 0.1244 & \\
\hline & approx & 0.1034 & -0.21 & 0.1191 & -0.65 & 0.1292 & 0.10 & 0.1246 & .28 & 0.1242 & 1.1 \\
\hline \multirow[t]{2}{*}{31} & exact & 0.0935 & & 0.1072 & & 152 & & 0.1115 & & 0.1117 & \\
\hline & prox & 0.0934 & .08 & 39 & -0.30 & 35 & 0.25 & 118 & 31 & 115 & 0.15 \\
\hline \multirow[t]{2}{*}{32} & exact & 0.0839 & & 0.0876 & & 0.0917 & & 0.0972 & & 0.09000 & \\
\hline & approx & 0.0840 & 0.10 & 0.0877 & 0.13 & & -0.18 & 9963 & -0.99 & 0.0901 & 0.15 \\
\hline \multirow[t]{2}{*}{$\overline{33}$} & exact & 0.0786 & & 0.0823 & & 0.0863 & & 0.0895 & & $0.0 \times 51$ & \\
\hline & approx & 0.0781 & -0.60 & 317 & -0.65 & 861 & -0.20 & 0.0899 & .45 & $0.0 \times 52$ & 10 \\
\hline \multirow[t]{2}{*}{34} & exact & 0.1007 & & 0.1071 & & 0.1151 & & 0.1224 & & 0.1132 & \\
\hline & approx & 0.1001 & -0.64 & 0.1064 & -0.67 & 0.1148 & -0.27 & 0.1231 & .56 & 0.1132 & 0.02 \\
\hline 35 & exact & 0.1028 & & 0.1089 & & 0.1154 & & 0.1219 & & 0.1122 & \\
\hline & approx & 0.1018 & -0.91 & 0.1078 & -1.01 & 149 & -0.37 & 0.1227 & .68 & 0.1123 & 0.077 \\
\hline$\overline{36}$ & exact & 0.0488 & & 0.0644 & & 0.0648 & & 0.0637 & & 0.0853 & \\
\hline & approx & 0.0484 & -0.83 & & -2.10 & 36 & -1.83 & 0.0627 & -1.50 & 0.0853 & 0.02 \\
\hline 37 & exact & 0.0390 & & 0.0487 & & 0.0490 & & 0.0482 & & 0.06 .35 & \\
\hline & approx & 0.0387 & -0.73 & 0.0477 & -2.11 & 0.0481 & -1.83 & 0.0475 & -1.50 & 0.06339 & 1.50 \\
\hline$\overline{38}$ & exact & 0.0587 & & 0.0864 & & 0.0869 & & 0.0852 & & 0.1136 & \\
\hline & approx & 0.0582 & -0.82 & 0.0849 & -1.77 & 0.0856 & -1.53 & 0.0842 & -1.23 & 0.1189 & $-0 .(f)$ \\
\hline 39 & exact & 0.0479 & & 0.0679 & & 0.0683 & & 0.0671 & & 0.0899 & \\
\hline & approx & 0.0476 & -0.56 & 0.0663 & -2.37 & 0.0669 & -2.07 & 0.0660 & -1.70 & 0.0846 & $-(1.315$ \\
\hline$\overline{40}$ & exact & 0.0417 & & 0.0598 & & 0.0602 & & 0.0592 & & $0.07 \times 5$ & \\
\hline & approx & 0.0415 & -0.44 & 0.0582 & -2.67 & 0.0588 & -2.33 & 0.0581 & -1.91 & 0.0782 & $-(1) .38$ \\
\hline
\end{tabular}

continues on the next page 
MR5 results continued

\begin{tabular}{|c|c|c|c|c|c|c|c|c|c|c|c|}
\hline \multirow{3}{*}{ Case } & \multirow{3}{*}{$\begin{array}{c}\text { Type } \\
\text { of } \\
\text { results }\end{array}$} & \multicolumn{10}{|c|}{ Reference task throughputs } \\
\hline & & \multicolumn{2}{|c|}{$\overline{T_{1}}$} & \multicolumn{2}{|c|}{$T_{2}$} & \multicolumn{2}{|c|}{$T_{3}$} & \multicolumn{2}{|c|}{$\overline{T_{4}}$} & \multicolumn{2}{|c|}{$T_{\mathbf{5}}$} \\
\hline & & thru & err\% & thru & err\% & thru & err\% & thru & err\% & thru & err\% \\
\hline 41 & $\begin{array}{c}\text { exact } \\
\text { approx }\end{array}$ & $\begin{array}{l}0.0333 \\
0.0332\end{array}$ & -0.32 & $\begin{array}{l}0.0482 \\
0.0468\end{array}$ & -2.93 & $\begin{array}{l}0.0485 \\
0.0472\end{array}$ & -2.56 & $\begin{array}{l}0.0477 \\
0.0467\end{array}$ & -2.10 & $\begin{array}{l}0.0628 \\
0.0625\end{array}$ & -0.37 \\
\hline$\overline{42}$ & $\begin{array}{l}\text { exact } \\
\text { approx }\end{array}$ & $\begin{array}{l}0.0500 \\
0.0503\end{array}$ & 0.56 & $\begin{array}{l}0.0500 \\
0.0503\end{array}$ & 0.56 & $\begin{array}{l}0.0500 \\
0.0503\end{array}$ & 0.56 & $\begin{array}{l}0.0500 \\
0.0504\end{array}$ & 0.78 & $\begin{array}{l}0.0500 \\
0.0504\end{array}$ & 0.78 \\
\hline 43 & $\begin{array}{c}\text { exact } \\
\text { approx }\end{array}$ & $\begin{array}{l}0.0500 \\
0.0501\end{array}$ & 0.16 & $\begin{array}{l}0.0500 \\
0.0501\end{array}$ & 0.16 & $\begin{array}{l}0.0500 \\
0.0501\end{array}$ & 0.16 & $\begin{array}{l}0.0500 \\
0.0501\end{array}$ & 0.16 & $\begin{array}{l}0.0500 \\
0.0501\end{array}$ & 0.16 \\
\hline 44 & $\begin{array}{l}\text { exact } \\
\text { approx }\end{array}$ & $\begin{array}{l}0.0333 \\
0.0334\end{array}$ & 0.31 & $\begin{array}{l}0.0333 \\
0.0334 \\
\end{array}$ & 0.31 & $\begin{array}{l}0.0333 \\
0.0334 \\
\end{array}$ & 0.31 & $\begin{array}{l}0.0333 \\
0.0334 \\
\end{array}$ & 0.31 & $\begin{array}{l}0.0333 \\
0.0335\end{array}$ & 0.59 \\
\hline 45 & $\begin{array}{c}\text { exact } \\
\text { approx }\end{array}$ & $\begin{array}{l}0.0997 \\
0.0990\end{array}$ & -0.72 & $\begin{array}{l}0.0997 \\
0.0990\end{array}$ & -0.72 & $\begin{array}{l}0.0997 \\
0.0990\end{array}$ & -0.72 & $\begin{array}{l}0.0997 \\
0.0990\end{array}$ & -0.72 & $\begin{array}{l}0.0997 \\
0.0990\end{array}$ & -0.72 \\
\hline 46 & $\begin{array}{l}\text { exact } \\
\text { approx }\end{array}$ & $\begin{array}{l}0.0500 \\
0.0500\end{array}$ & -0.08 & $\begin{array}{l}0.0500 \\
0.0500 \\
\end{array}$ & -0.08 & $\begin{array}{l}0.0500 \\
0.0500\end{array}$ & -0.08 & $\begin{array}{l}00500 \\
0.0500\end{array}$ & -0.08 & $\begin{array}{l}0.0500 \\
0.0500\end{array}$ & -0.08 \\
\hline 47 & $\begin{array}{l}\text { exact } \\
\text { approx }\end{array}$ & $\begin{array}{l}0.0500 \\
0.0499 \\
\end{array}$ & -0.14 & $\begin{array}{l}0.0500 \\
0.0499 \\
\end{array}$ & -0.14 & $\begin{array}{l}0.0500 \\
0.0499 \\
\end{array}$ & -0.14 & $\begin{array}{l}0.0500 \\
0.0499\end{array}$ & -0.14 & $\begin{array}{l}0.0500 \\
0.0499\end{array}$ & -0.14 \\
\hline 48 & $\begin{array}{l}\text { exact } \\
\text { approx }\end{array}$ & $\begin{array}{l}0.0333 \\
0.0333\end{array}$ & 0.00 & $\begin{array}{l}0.0333 \\
0.0333 \\
\end{array}$ & 0.00 & $\begin{array}{l}0.0333 \\
0.0333 \\
\end{array}$ & 0.00 & $\begin{array}{l}0.0333 \\
0.0333 \\
\end{array}$ & 0.00 & $\begin{array}{l}0.0333 \\
0.0333\end{array}$ & 0.00 \\
\hline 49 & $\begin{array}{l}\text { exact } \\
\text { approx }\end{array}$ & $\begin{array}{l}0.0766 \\
0.0760 \\
\end{array}$ & -0.79 & $\begin{array}{l}0.0766 \\
0.0760 \\
\end{array}$ & 0.79 & $\begin{array}{l}0.0766 \\
0.0760 \\
\end{array}$ & -0.79 & $\begin{array}{l}0.0766 \\
0.0760 \\
\end{array}$ & 1.79 & $\begin{array}{l}0.0766 \\
0.0760\end{array}$ & -0.79 \\
\hline 50 & $\begin{array}{c}\text { exact } \\
\text { approx }\end{array}$ & $\begin{array}{l}0.0479 \\
0.0470 \\
\end{array}$ & -1.94 & $\begin{array}{l}0.0479 \\
0.0470 \\
\end{array}$ & 1.94 & $\begin{array}{l}0.0479 \\
0.0470 \\
\end{array}$ & -1.94 & $\begin{array}{l}0.0479 \\
0.0470 \\
\end{array}$ & -1.94 & $\begin{array}{l}0.0479 \\
0.0470\end{array}$ & -1.94 \\
\hline 51 & $\begin{array}{c}\text { exact } \\
\text { approx }\end{array}$ & $\begin{array}{l}0.0485 \\
0.0477\end{array}$ & -1.65 & $\begin{array}{l}0.0485 \\
0.0477\end{array}$ & -1.65 & $\begin{array}{l}0.0485 \\
0.0477\end{array}$ & -1.65 & $\begin{array}{l}0.0485 \\
0.0477\end{array}$ & -1.65 & $\begin{array}{l}0.0485 \\
0.0477\end{array}$ & -1.65 \\
\hline 52 & $\begin{array}{l}\text { exact } \\
\text { approx }\end{array}$ & $\begin{array}{l}0.0099 \\
0.0099\end{array}$ & 0.00 & $\begin{array}{l}0.0099 \\
0.0099 \\
\end{array}$ & 0.00 & $\begin{array}{l}0.0099 \\
0.0099\end{array}$ & 0.00 & $\begin{array}{l}0.0099 \\
0.0099\end{array}$ & 0.00 & $\begin{array}{l}0.0099 \\
0.0099\end{array}$ & 0.00 \\
\hline $\mathbf{5 3}$ & $\begin{array}{c}\text { exact } \\
\text { approx } \\
\end{array}$ & $\begin{array}{l}0.0087 \\
0.0087 \\
\end{array}$ & -0.14 & $\begin{array}{l}0.0067 \\
0.0087\end{array}$ & -0.14 & $\begin{array}{l}0.0087 \\
0.0087 \\
\end{array}$ & -0.14 & $\begin{array}{l}0.0087 \\
0.0087 \\
\end{array}$ & -0.14 & $\begin{array}{l}0.0087 \\
0.0087 \\
\end{array}$ & -0.14 \\
\hline 54 & $\begin{array}{c}\text { exact } \\
\text { approx }\end{array}$ & $\begin{array}{l}0.0092 \\
0.0092 \\
\end{array}$ & .11 & $\begin{array}{l}0.0092 \\
0.0092 \\
\end{array}$ & -0.11 & & -0.11 & & -0.11 & $\begin{array}{l}0.0092 \\
0.0092 \\
\end{array}$ & -0.11 \\
\hline 55 & $\begin{array}{c}\text { exact } \\
\text { approx }\end{array}$ & $\begin{array}{l}0.0672 \\
0.0659 \\
\end{array}$ & .94 & $\begin{array}{l}0.0660 \\
0.0649 \\
\end{array}$ & .77 & $\begin{array}{l}0.0647 \\
0.0637 \\
\end{array}$ & -1.57 & $\begin{array}{l}0.0636 \\
0.0627 \\
\end{array}$ & .43 & $\begin{array}{l}0.0613 \\
0.0606 \\
\end{array}$ & -1.14 \\
\hline$\overline{56}$ & $\begin{array}{l}\text { exact } \\
\text { approx }\end{array}$ & $\begin{array}{l}0.0560 \\
0.0547 \\
\end{array}$ & -2.46 & $\begin{array}{l}0.0555 \\
0.0543 \\
\end{array}$ & -2.13 & $\begin{array}{l}0.0550 \\
0.0540 \\
\end{array}$ & -1.83 & $\begin{array}{l}0.0545 \\
0.0536 \\
\end{array}$ & 1.62 & $\begin{array}{l}0.0535 \\
0.0528 \\
\end{array}$ & -1.28 \\
\hline 57 & $\begin{array}{c}\text { exact } \\
\text { approx }\end{array}$ & $\begin{array}{l}0.0689 \\
0.0668 \\
\end{array}$ & -3.13 & $\begin{array}{l}0.0633 \\
0.0617 \\
\end{array}$ & -2.42 & $\begin{array}{l}0.0585 \\
0.0574 \\
\end{array}$ & -1.88 & $\begin{array}{l}0.0545 \\
0.0537 \\
\end{array}$ & -1.53 & $\begin{array}{l}0.0506 \\
0.0500 \\
\end{array}$ & -1.11 \\
\hline 58 & $\begin{array}{c}\text { exact } \\
\text { approx }\end{array}$ & $\begin{array}{l}0.0495 \\
0.0486 \\
\end{array}$ & 98 & $\begin{array}{l}0.0521 \\
0.0511 \\
\end{array}$ & .79 & $\begin{array}{l}0.0549 \\
0.0540 \\
\end{array}$ & .58 & $\begin{array}{l}0.0582 \\
0.0574 \\
\end{array}$ & -1.43 & $\begin{array}{l}0.0617 \\
0.0610 \\
\end{array}$ & -1.08 \\
\hline 59 & $\begin{array}{c}\text { exact } \\
\text { approx } \\
\end{array}$ & $\begin{array}{l}0.0588 \\
0.0577 \\
\end{array}$ & -1.91 & $\begin{array}{l}0.0586 \\
0.0578 \\
\end{array}$ & -1.39 & $\begin{array}{l}0.0584 \\
0.0579 \\
\end{array}$ & -0.97 & $\begin{array}{l}0.0583 \\
0.0578 \\
\end{array}$ & -0.70 & $\begin{array}{l}0.0579 \\
0.0577\end{array}$ & -0.32 \\
\hline 60 & $\begin{array}{c}\text { exact } \\
\text { approx }\end{array}$ & $\begin{array}{l}0.0644 \\
0.0633 \\
\end{array}$ & -1.66 & $\begin{array}{l}0.0618 \\
0.0612 \\
\end{array}$ & -1.03 & $\begin{array}{l}0.0596 \\
0.0592\end{array}$ & -0.61 & $\begin{array}{l}0.0575 \\
0.0573 \\
\end{array}$ & -0.38 & $\begin{array}{l}0.0555 \\
0.0554\end{array}$ & -0.11 \\
\hline
\end{tabular}


MR5 results continued

\begin{tabular}{|c|c|c|c|c|c|c|c|c|c|c|c|}
\hline \multirow{3}{*}{ Case } & \multirow{3}{*}{$\begin{array}{c}\text { Type } \\
\text { of } \\
\text { results }\end{array}$} & \multicolumn{10}{|c|}{ Reference task throughputs } \\
\hline & & \multicolumn{2}{|c|}{$\overline{T_{1}}$} & \multicolumn{2}{|c|}{$\overline{T_{2}}$} & \multicolumn{2}{|c|}{$T_{3}$} & \multicolumn{2}{|c|}{$\overline{T_{4}}$} & \multicolumn{2}{|c|}{$T_{5}$} \\
\hline & & thru & err\% & thru & err\% & thru & err\% & thru & err\% & Thru & err \\
\hline \multirow[t]{2}{*}{61} & exact & 0.0304 & & 0.0301 & & 0.0298 & & 0.0295 & & 0.0289 & \\
\hline & approx & 0.0303 & -0.16 & 0.0301 & -0.14 & 0.0297 & -0.12 & 0.0295 & -0.11 & 0.0289 & -0.106 \\
\hline \multirow[t]{2}{*}{62} & exact & 0.0472 & & 0.0408 & & 0.0358 & & 0.0320 & & $0.02 \times 6$ & \\
\hline & approx & 0.0470 & -0.45 & 0.0406 & -0.34 & 0.0357 & -0.25 & 0.0319 & -0.20 & 0.0285 & -0.15 \\
\hline \multirow[t]{2}{*}{63} & exact & 0.0534 & & 0.0476 & & 0.0423 & & 0.0369 & & $0.036 t^{2}$ & \\
\hline & approx & 0.0528 & -1.16 & 0.0474 & -0.41 & 0.0419 & -1.06 & 0.0366 & -0.80 & $0.03 t i 1$ & -0.42 \\
\hline \multirow[t]{2}{*}{64} & exact & 0.0425 & & 0.0389 & & 0.0345 & & 0.0318 & & 0.0314 & \\
\hline & approx & 0.0420 & -1.21 & 0.0388 & -0.19 & 0.0344 & -0.38 & 0.0315 & -1.01 & 0.0313 & $-(1) .4 !$ \\
\hline \multirow[t]{2}{*}{65} & exact & 0.0237 & & 0.0242 & & 0.0224 & & 0.0210 & & 0.0208 & \\
\hline & approx & 0.0235 & -0.98 & 0.0242 & 0.09 & 0.0224 & 0.02 & 0.0207 & -1.33 & 0.0207 & $-0.6 \mathrm{tit}$ \\
\hline \multirow[t]{2}{*}{66} & exact & 0.0457 & & 0.0386 & & 0.0342 & & 0.0318 & & 0.0315 & \\
\hline & approx & 0.0460 & 0.75 & 0.0385 & -0.41 & 0.0341 & -0.41 & 0.0316 & -0.93 & 0.0313 & $-(1) .40$ \\
\hline \multirow[t]{2}{*}{67} & exact & 0.0505 & & 0.0417 & & 0.0364 & & 0.0326 & & 0.0244 & \\
\hline & approx & 0.0510 & 0.97 & 0.0414 & -0.71 & 0.0362 & -0.69 & 0.0322 & -1.15 & 0.0243 & -01.1 .1 \\
\hline \multirow[t]{2}{*}{68} & exact & 0.0367 & & 0.0319 & & 0.0288 & & 0.0266 & & 0.0125 & \\
\hline & approx & 0.0370 & 0.73 & 0.0317 & -0.57 & 0.0287 & -0.47 & 0.0265 & -0.41 & 0.0125 & -0.23 \\
\hline \multirow[t]{2}{*}{69} & exact & 0.0287 & & 0.0256 & & 0.0234 & & 0.0219 & & 0.0102 & \\
\hline & approx & 0.0291 & 1.34 & 0.0255 & -0.71 & 0.0232 & -0.65 & 0.0218 & -0.50 & 0.0102 & - (1).08 \\
\hline \multirow[t]{2}{*}{70} & exact & 0.0239 & & 0.0213 & & 0.0202 & & 0.0191 & & 0.0096 & \\
\hline & approx & 0.0240 & 0.53 & 0.0213 & 0.31 & 0.0201 & -0.82 & 0.0190 & -0.62 & 0.0096 & -0.08 \\
\hline \multirow[t]{2}{*}{71} & exact & 0.0216 & & 0.0196 & & 0.0187 & & 0.0178 & & 0.0099 & \\
\hline & approx & 0.0216 & 0.24 & 0.0197 & 0.49 & 0.0186 & -0.78 & 0.0176 & -0.69 & 0.0099 & -0.12 \\
\hline \multirow[t]{2}{*}{72} & exact & 0.0229 & & 0.0207 & & 0.0196 & & 0.0152 & & 0.0101 & \\
\hline & approx & 0.0231 & 0.79 & 0.0207 & 0.35 & 0.0194 & -1.12 & 0.0150 & -0.93 & 0.0101 & -0.21 \\
\hline \multirow[t]{2}{*}{$\overline{73}$} & exact & 0.0244 & & 0.0183 & & 0.0205 & & 0.0156 & & 0.0102 & \\
\hline & approx & 0.0250 & 2.15 & 0.0181 & -0.99 & 0.0202 & -1.31 & 0.0154 & -1.15 & 0.0102 & -0.28 \\
\hline \multirow[t]{2}{*}{74} & exact & 0.0721 & & 0.0373 & & 0.0430 & & 0.0378 & & 0.0158 & \\
\hline & approx & 0.0735 & 1.92 & 0.0367 & -1.50 & 0.0423 & -1.52 & 0.0371 & -1.97 & 0.0157 & -0.39 \\
\hline 75 & exact & 0.0427 & & 0.0275 & & 0.0306 & & 0.0280 & & 0.0131 & \\
\hline & approx & 0.0442 & 3.56 & 0.0271 & -1.49 & 0.0303 & -1.09 & 0.0278 & -0.84 & 0.0131 & -0.39 \\
\hline 76 & exact & 0.0307 & & 0.0222 & & 0.0244 & & 0.0227 & & 0.0106 & \\
\hline & approx & 0.0313 & 1.71 & 0.0219 & -1.49 & 0.0242 & -0.89 & 0.0225 & -0.75 & 0.0106 & -0.13 \\
\hline 77 & exact & 0.0252 & & 0.0188 & & 0.0210 & & 0.0197 & & 0.0097 & \\
\hline & approx & 0.0257 & 1.91 & 0.0187 & -0.62 & 0.0208 & -1.08 & 0.0196 & -0.86 & 0.0097 & -0.14 \\
\hline 78 & exact & 0.0226 & & 0.0171 & & 0.0190 & & 0.0162 & & 0.0111 & \\
\hline & approx & 0.0233 & 2.92 & 0.0170 & -0.85 & 0.0188 & -1.26 & 0.0160 & -1.09 & 0.0111 & -0.27 \\
\hline 79 & exact & 0.0269 & & 0.0225 & & 0.0219 & & 0.0206 & & 0.0131 & \\
\hline & approx & 0.0272 & 1.23 & 0.0222 & -1.23 & 0.0218 & -0.69 & 0.0205 & -0.46 & 0.0130 & $-(1.10$ \\
\hline 80 & exact & 0.0510 & & 0.0479 & & 0.0480 & & 0.0496 & & 0.0511 & \\
\hline & approx & 0.0510 & 0.09 & 0.0479 & 0.06 & 0.0480 & 0.03 & 0.0494 & -0.26 & 0.0511 & $0.06 ;$ \\
\hline
\end{tabular}


MR5 results continued

\begin{tabular}{|c|c|c|c|c|c|c|c|c|c|c|c|}
\hline \multirow{3}{*}{ Case } & \multirow{3}{*}{$\begin{array}{l}\text { Type } \\
\text { of } \\
\text { results }\end{array}$} & \multicolumn{10}{|c|}{ Reference task throughputs } \\
\hline & & \multicolumn{2}{|c|}{$\overline{T_{1}}$} & \multicolumn{2}{|c|}{$\overline{T_{2}}$} & \multicolumn{2}{|c|}{$\overline{T_{3}}$} & \multicolumn{2}{|c|}{$\overline{T_{4}}$} & \multicolumn{2}{|c|}{$\overline{T_{5}}$} \\
\hline & & thru & err\% & thru & err\% & thru & err\% & thru & err\% & thru & err\% \\
\hline \multirow[t]{2}{*}{$8 !$} & exact & 0.0249 & & 0.0243 & & 0.0243 & & 0.0247 & & 0.0249 & \\
\hline & approx & 0.0249 & 0.00 & 0.0244 & 0.32 & 0.0244 & 0.19 & 0.0247 & -0.04 & 0.0249 & -0.08 \\
\hline \multirow[t]{2}{*}{82} & exact & 0.0261 & & 0.0254 & & 0.0255 & & 0.0215 & & 0.0236 & \\
\hline & approx & 0.0262 & 0.37 & 0.0254 & 0.06 & 0.0255 & -0.08 & 0.0214 & -0.22 & 0.0236 & -0.23 \\
\hline \multirow[t]{2}{*}{$\overline{83}$} & exact & 0.0275 & & 0.0267 & & 0.0268 & & 0.0180 & & 0.0217 & \\
\hline & approx & 0.0277 & 0.42 & 0.0267 & 0.00 & 0.0268 & -0.08 & 0.0179 & -0.21 & 0.0216 & -0.34 \\
\hline \multirow[t]{2}{*}{84} & exact & 0.0296 & & 0.0287 & & 0.0288 & & 0.0134 & & 0.0182 & \\
\hline & approx & $0.0299^{\circ}$ & 0.50 & 0.0286 & -0.10 & 0.0287 & -0.15 & 0.0134 & -0.16 & 0.0182 & -0.31 \\
\hline \multirow[t]{2}{*}{85} & exact & 0.0309 & & 0.0298 & & 0.0299 & & 0.0136 & & 0.0125 & \\
\hline & approx & 0.0310 & 0.48 & 0.0298 & -0.13 & 0.0299 & -0.17 & 0.0136 & -0.17 & 0.0125 & -0.16 \\
\hline \multirow[t]{2}{*}{86} & exact & 0.0335 & & 0.0322 & & 0.0323 & & 0.0076 & & 0.0088 & \\
\hline & approx & 0.0336 & 0.34 & 0.0321 & -0.12 & 0.0323 & -0.16 & 0.0076 & -0.04 & 0.0088 & -0.07 \\
\hline \multirow[t]{2}{*}{87} & xact & 0.0218 & & 0.0213 & & 0.0209 & & 0.0205 & & 0.0202 & \\
\hline & approx & 0.0215 & -1.50 & 0.0212 & -0.17 & 0.0209 & 0.22 & 0.0205 & 0.27 & 0.0202 & 0.21 \\
\hline \multirow[t]{2}{*}{88} & exact & 0.0267 & & 0.0258 & & 0.0251 & & 0.0245 & & 0.0240 & \\
\hline & approx & 0.0262 & -1.70 & 0.0257 & -0.13 & 0.0251 & 0.23 & 0.0246 & 0.30 & 0.0240 & 0.23 \\
\hline \multirow[t]{2}{*}{89} & exact & 0.0265 & & 0.0257 & & 0.0251 & & 0.0245 & & 0.0240 & \\
\hline & approx & 0.0261 & -1.31 & 0.0257 & 0.03 & 0.0251 & 0.17 & 0.0246 & 0.20 & 0.0241 & 0.15 \\
\hline \multirow[t]{2}{*}{90} & exact & 0.0095 & & 0.0094 & & 0.0091 & & 0.0089 & & 0.0088 & \\
\hline & approx & 0.0092 & -3.07 & 0.0092 & -1.46 & 0.0091 & 0.25 & 0.0089 & 0.50 & 0.0088 & 0.30 \\
\hline \multirow[t]{2}{*}{91} & exact & 0.0128 & & 0.0125 & & 0.0121 & & 0.0117 & & 0.0115 & \\
\hline & approx & 0.0129 & 0.66 & 0.0124 & -0.81 & 0.0121 & -0.03 & 0.0117 & 0.08 & 0.0115 & 0.06 \\
\hline \multirow[t]{2}{*}{92} & exact & $0.004 !$ & & 0.0038 & & 0.0034 & & 0.0029 & & 0.0028 & \\
\hline & зрprox & 0.0042 & 2.06 & 0.0038 & -1.26 & 0.0034 & -0.70 & 0.0029 & -0.17 & 0.0028 & -1.27 \\
\hline \multirow[t]{2}{*}{93} & exact & 0.0037 & & $0.0 \overline{034}$ & & 0.0034 & & 0.0028 & & 0.0027 & \\
\hline & approx & 0.0035 & -3.74 & 0.0034 & 0.78 & 0.0034 & 0.60 & 0.0028 & 0.18 & 0.0027 & -0.84 \\
\hline \multirow[t]{2}{*}{94} & exact & 0.0045 & & 0.0040 & & 0.0041 & & 0.0032 & & 0.0031 & \\
\hline & approx & 0.0044 & -1.75 & 0.0040 & 0.12 & 0.0041 & 0.15 & 0.0032 & 0.19 & 0.0031 & -0.45 \\
\hline \multirow[t]{2}{*}{95} & exact & 0.0039 & & 0.0036 & & 0.0037 & & 0.0029 & & 0.0029 & \\
\hline & approx & 0.0039 & -0.21 & 0.0036 & 0.16 & 0.0037 & -0.38 & 0.0030 & 0.10 & 0.0029 & -0.52 \\
\hline
\end{tabular}




\section{Bibliography}

[AB83] S. C. Agrawal and J. P. Buzen. The aggregate server method for analyzing serialization delays in computer systems. ACM Trans. on Computer Systems, 1(2):116-143, May 1983.

[ABS84] S. C. Agrawal, J. P. Buzen, and A. W. Shum. Response time preservation: a general techique for developing approximate algorithms for QN. In Proc. 1984 ACM SIGMETRICS Conference on Measurement and Modeling, Cambridge, MA, August 1984.

[Agr85] S. C. Agrawal. Metamodeling: A Study of Approximations in Queueing Models. MIT Press Series in Computer Systems, 1985.

[AI89] H. H. Ammar and S. M. R. Islam. Time scale decomposition of a class of GSPN models. IEEE Transactions on Computers, C-15(6), June 1989.

[Aky87] I. F. Akyildiz. Exact product form solution for queueing networks with blocking. IEEE Transactions on Computers, C-36(1), January 1987.

[Aky88] I. F. Akyildiz. Mean value analysis for blocking queueing networks. IEEE Transactions on Software Eng., SE-14(4), April 1988.

[Aky89] I. F. Akyildiz. Product form approximations for queueing networks with multiple servers. IEEE Transactions on Computers, C-39(1), January 1989.

[AMBC84] M. Ajmone Marsan, G. Balbo, and G. Conte. A class of generalized stochastic Petri Nets for the performance evaluation of multiprocessor systems. A C:M Trans. on Computer Systems, 2(2):93-122, May 1984. 
[AMBCC87] M. Ajmone Marsan, G. Balbo, G. Chiola, and G. Conte. Applicability of stochastic petri nets to performance modelling. In 2nd Int. Wkshop on Applied Mathematics and Performance/Reliability Models of Computer/Communication Systems, pages 517-534, Rome, Italy, May 1987.

[AMC86] M. Ajmone Marsan and G. Chiola. On petri nets with deterministic and exponential transition firing times. In Proc.7th European Workshop on Application and Theory of PN, Oxford, England, June 1986.

[Bar79] Y. Bard. Some extensions to multiclass queueing network analysis. In A. Butrimenko M. Arato and E. Gelenbe, editors, Performance of computer systems. North-Holland, 1979.

[BCMP75] F. Basket, K. M. Chandy, R. R. Munz, and F. G. Palacios. Open, closed and mixed networks of queues with different classes of customers. Journal of $A C M$, 22(2):248-260, April 1975.

[BKLC84] R. M. Bryant, A. E. Krzesinski, M. S. Lakshmi, and K. M. Chandy. The MVA priority approximation. ACM Transactions on Computer Systems, 2(4):335359, November 1984.

[Buz71] J. P. Buzen. Queueing Network Models of Multiprogramming. PhD thesis, Harvard University, Cambridge, MA, 1971.

[CdL89] A. E. Conway, E. de Souza e Silva, and S. S. Lavenberg. Mean value analysis by chain of product form queueing networks. IEEE Trans. Computers, 38(3):432$442,1989$.

[CG86] A. E. Conway and N. D. Georganas. Recal - a new efficient algorithm for the exact analysis of multiple-chain closed queueing networks. Journal of $A C M$, 33:768-791, 1986.

[Che82] D. R. Cheriton. The Toth Operating System: multi-process structuring and portability. Elsevier, New York, 1982. 
[Che84] D. R. Cheriton. The V kernel: A software base for distributed systems. IEEE: Software, 1(2):19-42, April 1984.

[Chi87] G. Chiola. GreatSPN Users' Manual, Version 1.3. Dipartimento di Informatica, Università degli Studi di Torino, corso Svizzera 185, 10149 Torino, Italy, September 1987.

[CHT77] K. M. Chandy, J. H. Howardand, and D. F. Towsley. Product form and local balance in queueing networks. Journal of $A C M, 24(2): 250-263,1977$.

[CHW75] K. M. Chandy, U. Hertzog, and L. Woo. Parametric analysis of queucing networks. IBM J.Res.Develop., 19(1):36-42, January 1975.

[Cia89] G. Ciardo. Analysis of Large Stochastic Pelri Net Models. PhD thesis, Department of Computer Science, Duke University, 1989.

[CN82] K.M. Chandy and D. Neuse. Linearizer: a heuristic algorithm for queueing network models of computing systems. Communications of the $A C M, 25(2): 126$ 134, February 1982.

[Cou77] P. J. Courtois. Decomposability: Queueing and Computer Applications. Academic Press, New York, 1977.

[Cou85] P. J. Courtois. On time and space decomposition of complex structiares. Communications of the $A C M, 28(6): 590-603$, June 1985.

[dLM84] E. de Souza e Silva, S. S. Lavenberg, and R. R. Muntz. A peripective on iterative methods for the approximate analysis of closed queueing networks. In G. Iazeolla, P.J. Courtois, and A Hordijk, editors, Mathematical Computer Performance and Reliability. Elsevier Science Publishers B.V., North-Holland, 1984.

[dLM86] E. de Souza e Silva, S. S. Lavenberg, and R .R. Muntz. A clustering approximation technique for queueing network models with a large number of chains. IEEE Trans. Computers, 35(5):419-430, 1986. 
[dM87] E. de Souza e Silva and R. R. Muntz. Approximate solutions for a class of nonproduct form queueing network models. Performance Evaluation, 7:221-242, 1987.

[DOD83] DOD. Reference Manual for the Ada Programming Language ANSI/MIL.STD 1815. United States Department of Defense, January 1983.

[EL 88$] \quad$ D. L. Eager and J. N. Lipscomb. The amva priority approximation. Performance Evaluation, 8:173-193, 1988.

[ES84] D. L. Eager and K. C. Sevcik. An analysis of an approximation algorithm for queueing networks. Performance Evaluation, 4:275-284, 1984.

[FC87] B. N. Feinberg and S. S. Chiu. A method to calculate steady-state distributions of large Markov chains by aggregating states. Operations Research, 35(2):282290, March-April 1987.

[Fer86] D. Ferrari. Considerations on the insularity of performance evaluation. IEEE Trans. on Software Eng., SE-12(6):678-683, June 1986.

[Fon89] M. J. Fontenot. Software congestion, mobile servers, and the hyperbolic model. IEEE Trans. Software Eng., 15:947-962, 1989.

[GC84] N. D. Gammage and L. M. Casey. The software architecture of a distributed processing system. In Proc. 4th Int. Conf. on Distributed Computing Systems, pages 414-431, May 1984. IEEE Catalog 84CH2021-4.

[Gen87] W. M. Gentleman. Using the Harmony operating system. Technical Report ERB-966, NRCC No. 27469, National Research Council of Canada, Ottawa, 1987.

[Gig87] J. Giglmayr. Analysis of stochastic Petri Nets by the decomposition of the transition rate matrix. NTZ Archiv (Germany), 1987. Part 1: Vol. 9, No. 5, pp. 115-120; Part 2: Vol. 9, No. 6, pp. 147-152. 
[Hoa85] C. A. R. Hoare. Communicating Sequential Processes. Prentice-Hall International, UK, Ltd., London, England, 1985.

[Hoa88] series editor Hoare, C. A. R. INMOS Limited Occam 2 Reference Manual. Prentice Hall International, 66 Wood Land End, Hemel Hempstead, Hertfordshire, England HP2 4RG, 1988.

[HT82] P. Heidelberger and K. S. Trivedi. Queueing network models for parallel processing with asynchronous tasks. IEEE Transactions on Computers, C-31(11), November 1982.

[HT83] P. Heidelberger and K. S. Trivedi. Analytic queueing models for programs with internal concurrency. IEEE Transactions on Computers, C-32(1), January 1983.

[HV87] M. A. Holliday and M. K. Vernon. A generalized timed Petri Net model for performance analysis. IEEE Transactions on Software Engincering, SE13(12):1297-1310, December 1987.

[Jac57] J. R. Jackson. Networks of waiting lines. Operations Research, 5:518-521, July 1957.

[Jac63] J. R. Jackson. Jobshop-like queueing systems. Management Science, 10(1):131 142, October 1963.

[JL82] P. A. Jacobson and E. D. Lazowska. Analyzing queueing networks with simultaneous resource possession. Communications of the ACM, 25(2):142-151, February 1982.

[JL83] P. A. Jacobson and E. D. Lazowska. A reduction technique for evaluating queueing networks with serialization delays. In Performance '83, Proc. of $9 t h$ Int. Symp. on Comp. Perf. Mod., Meas., and Eval., pages 45-60, May 25-27 1983. 
[Lav80] S. S. Lavenberg. Stationary state probabilitiesat arrival instants for closed queueing networks with multiple types of customers. Journal of Appl.Prob., 17:1048-1061, 1980.

[Lav89] S. S. Lavenberg. A perspective on queueing models of computer performance. Performance Evaluation, 10:53-76, 1989.

[LZGS84] E. D. Lazowska, J. Zahorjan, G. S. Graham, and K. C. Sevcik. Quantitative System Performance. Prentice-Hall, Inc., Englewood Cliffs, N.J. 07632, 1984.

[Mar79] R. A. Marie. An approximate analytical method for general QN. IEEE Trans. on Software Eng., SE-5(5):530-538, September 1979.

[Mil80] R. Milner. A calculus of communicating systems. Lecture Notes in Computer Science 92, 1980.

[MMS85] J. F. Meyer, M. Movaghar, and W. H. Sanders. Stochastic activity networks: Structure, behaviour, and application. In International Workshop on Timed Petri Nets, pages 106-115, Torino, Italy, July 1985.

[Mol82] M. K. Molloy. Performance analysis using stochastic Petri Nets. IEEE Trans. on Computers, C-31:913-917, September 1982.

[Mol85] M. K. Molloy. Fast bounds for stochastic Petri Nets. Int. Workshop on Timed Petri Nets, Torino, Italy, pages 244-249, July 1985.

[Mol89] M. K. Molloy. Fundamentals of Performance Modeling. Macmillan P. C., New York, 1989.

[MWNP88] J. W. Miernik, C. M. Woodside, J. E. Neilson, and D. C. Petriu. Throughput of stochastic rendezvous networks with caller-specific service and processor contention. In Proc. IEEE Infocom '88, pages 1040-1049, March 1988.

[MWNP89] J. W. Miernik, C. M. Woodside, J. E. Neilson, and D. C. Petriu. Performance of stochastic rendezvous networks with priority tasks. In T.Hasegawa, H.Takagi, and Y.Takahashi, editors, Performance of Distributed and Parallel Systems, pages 511-525. Elsevier Science Publisher, 1989. 
[Nat80] S. Natkin. Les réseaux de Petri stochastiques et leur application a l'evaluation des systemes informatiques. PhD thesis, CNAM Paris, France, 1980. Thèse de Docteur īngenieur.

[NC82] D. Neuse and K. M. Chandy. Ham: The heuristic aggregation method for solving general closed queueing network models of computer systems. Performunce Evaluation Review, 11(4):195-212, 1982.

[NWMP87] J. E. Neilson, C. M. Woodside, J. W. Miernik, and D. C. Petriu. Stochastic rendezvouts network performance - fast, first-order approximations. Technical report, School of Computer Science, Carleton University, Ottawa, Canada KIS 5B6, August 1987.

[OP88] R. O. Onvural and H. G. Perros. Approximate throughput analysis of cyclic queueing networks with finite buffers. IEEE Transactions on Sofiware Eng., SE-15, June 1988.

[OP89] R. O. Onvural and H. G. Perros. Some equivalencies between closed queueing networks with blocking. Performance Evaluation, 9, June 1989.

[Per84] H. G. Perros. Queueing network with blocking: a bibliography. ACM Performance Evaluation Review, August 1984.

[PNL88] H. G. Perros, A. A. Nilsson, and Y. C. Liu. Approximate analysis of product form type queueing netwotks with blocking and deadlock. Performaner Evaluation, 8:19-39, February 1988.

[PS87] D. Peng and K. G. Shin. Modeling of concurrent task execution in a distributed system for real-time control. IEEE Trans. on Computers, C-36(4):500-516, April 1987.

[PWN89] D. C. Petriu, C. M. Woodside, and J. E. Neilson. Arrival instant probabilities for single client/server rendezvous networks based on Markov Chain task directed aggregation. Report SCE-89-32, Dept. of Systems and Computer Engineering, Carleton University, Ottawa, Canada KIS 5B6, December 1989. 
[Rari84] S. Radia. A Markov model for distributed communicating sequential processes. In Performance '84, Proceedings of the 10th International Symposium on Compuler Performance, pages 3-16. North Holland, 1984.

[Ras87] R. F. Rashid. Fum RIG to Accent to Mach: the evolution of a network operating system. Technical report, Computer Science Dept., Carnegie Mellon University, U.S.A., 1987.

[Rri76] M. Reiser. Interactive modeling of computer systems. IBM System Journal, 15(4):309-327, 1976.

[Rej79] M. Reiser. A queueing network analysis of computer communication networks with window flow control. IEEE Trans. on Communications, COM-27:11991209, August 1979.

[RLS0] M. Reiser and S. S. Lavenberg. Mean-value analysis of closed multichain queueing networks. Journal of ACa!, 27(2):313-322, April 1980.

[Rol88] J. A. Rolia. Performance estimates for systems with software servers: The lazy boss method. Proceedings VIII SCCC International Conference on Computer Science, pages $25-43$, July 1988.

[RPS4] P. R. Razouk and C. V. Phelp3. Performance analysis using timed petri nets. In International Conf. on Parallel Processing, pages 126-129, August 1984.

[SA61] H. A. Simon and A. Ando. Aggregation of variables in dynamic systems. Econometrica, 29:111-138, 1961.

[Sau83] C. H. Sauer. Computational algorithms for state dependent queueing networks. ACM Trans. on Computer Systems, 1(1):67-92, February 1983.

$\therefore$ C. H. Sauer and K. M. Chandy. Computer Systems Performance Modeling. Prentice-Hall, Inc., Englewood Cliffs, New Jersey 07632, 1981.

$\therefore$ : $h i$. J. Schweitzer. Approximate analysis of multiclass closed networks of queues. In Proc. International Conference on Stochastic Control and Optimization, Amsterdam, 1979. 
[Sch84] P. J. Schweitzer. Aggregation methods for large Markov chains. In Mathrmatical Computer Performance and Reliability, pages 275-286, North-Holland, 1984. Elsevier Science Publishers B.V.

[SD84] R. Suri and G. W. Diehl. A variable buffer size model and its use in analytical closed queueing networks with blocking. In 1984 ACM SIGMETRICS (onference on Measurement and Modeling of Computer Systems, pages 134-1.4. 1984.

[Sev77] K. C. Sevcik. Priority scheduling disciplines in queueing network model of computer systems. In Proc. of the IFIP Congress '77, pages 565-570. North Holland, 1977.

[SI82] S.Balsamo and G. Iazeolla. An extension of norton's theorem for queueing networks. IEEE Trans. on Software Engineering, SE-8(4):298-305, April 1982.

[Sif79] J. Sifakis. Performance evaluation of systems using petri nets. In G.Goos and J.Artmanis, editors, Net Theory and Applications. Springer Verlag, New York, 1979.

[SM87] W. H. Sanders and J. F. Meyer. Performance variable driven construction inethods for stochastic activity networks. In 2nd Int. Whshop on Applied Mathematics and Performance/Reliability Models of Computer/Communication Systems. University of Rome II, May 1987.

[Smi90] Connie U. Smith. Performance Engineering of Software Systems. AddisonWesley Publishing Company, 1990.

[Ste78] W. J. Stewart. A comparison of numerical techniques in markov modeling. Communications of the ACM, 21(2):144-152, February 1978.

[Tak75] Y. Takahashi. A lumping method for numerical calculations of stationary distributions of markov chains. Technical Report B-18, Dept. of Information Sciences, Tokyo Institute of Technology, Tokyo, 1975. 
[TB85] A. Thomasian and P. Bay. Performance analysis of task systems using a queueing network model. In International Workshop on Timed Petri Nets, pages 234-241, Torino, Italy, July 1985.

[Van78] H. Vantilborgh. Exact aggregation in exponential queueing networks. Journal of $A C M, 25(4): 620-629$, October 1978.

[Van85] H. Vantilborgh. Aggregation with an error of o $\left(\epsilon^{2}\right)$. Journal of $A C M, 32(1): 162-$ 190, January 1985.

[VZL87] M. K. Vernon, J. Zahorjan, and E. D. Lazowska. A comparison of performance Petri Nets and Queueing Network models. In S. Fida and G. Pujolle, editors, Modelling Techniques and Performance Evaluation, pages 191-202. Elsevier Science Publishers B.V. (North-Holland), 1987.

[WNM+88] C. M. Woodside, J. E. Neilson, J. W. Miernik, D. C. Petriu, and R. Constantin. Performance of concurrent rendezvous systems with complex pipeline structures. In Proc. 4th International Conference in Modelling Techniques and Tools for Computer Performance Evaluation, pages 361-378, September 1988.

[WNPM89] C. M. Woodside, J. E. Neilson, D. C. Petriu, and S. Majumdar. The rendezvous network model for performance synchronous multi-tasking distributed software. Sce-89-8, Dept. of Systems \& Computer Engineering and School of Computer Science, Carleton University, March 1989.

[Woo86] C. M. Woodside. Throughput calculations for basic stochastic rendezvous networks. Technical Report 86-10, Department of Systems and Computer Engineering, Carleton University, Ottawa, May 1986.

[Woo89] C. M. Woodside. Throughput calculation for basic stochastic rendezvous networks. Performance Evaluation, 9:143-160, 1989.

[Zah83] J. Zahorjan. Workload representations in queueing models of computer systems. Report 83-03-01, Dept.of Computer Science, University of Washington, Seattle, 1983. 
[ZES88] J. Zahorjan, D. L. Eager, and H. M. Sweillam. Accuracy, speed, and con vergence of approximate value analysis. Performance Evaluation, s:255-270, 1988.

[ZS86] Pamela Zave and William Schell. Salient features of an executable sperilication language and its environment. IEEE Trans. on Softurare Eng., SE-1:2(2):31:2 325, February 1986.

[Zub80] W. M. Zuberek. Timed Petri Nets and preliminary performance evaluation. Proc. 7th Annual Symposium Computer Architecture, pages 85-96, 1980. 
